\title{
The Nutritional Implications of Partner Switching in the Cnidarian-Dinoflagellate Symbiosis
}

\author{
Jennifer L. Matthews
}

\author{
A thesis submitted to the \\ Victoria University of Wellington \\ in fulfilment of the requirements for the degree of \\ Doctor of Philosophy
}

Victoria University of Wellington

2017 


\begin{abstract}
Reef-building corals form a symbiosis with phototrophic dinoflagellates of the genus Symbiodinium. Specificity in the host-symbiont partnership is widespread, despite the potential for flexibility in endosymbiont community composition to provide a mechanism of environmental acclimatisation and adaptation. The potential for partner switching may be linked to the nutritional flux between the two partners, with optimal nutritional exchange determining success. Further research is therefore necessary to determine how novel symbiont types (i.e. not originally detected in the host) affect the nutritional biology of the cnidarian host, and ultimately the capacity for the evolution of novel associations in the cnidariandinoflagellate symbiosis. The specific objectives were: 1) to design an effective method of providing aposymbiotic host organisms for experimental symbiont colonisation studies; 2) to determine how colonisation with a novel symbiont affects the gene-to-metabolite response of the host; 3) to deduce how the quantity and identity of translocated photosynthetic products from symbiont to host is affected by the symbiont type; 4) to determine whether natural differences in the Symbiodinium community composition affect the metabolite profile of the same cnidarian host.
\end{abstract}

Chapter 2 demonstrates that menthol-induced bleaching effectively and efficiently provides experimental aposymbiotic (i.e. symbiont-free) sea anemones (Aiptasia sp.) for colonisation studies. The menthol treatment produced aposymbiotic hosts within just 4 weeks (97-100\% symbiont loss), and the condition was maintained long after treatment when anemones were held under a standard light:dark cycle. The ability of Aiptasia to form a stable symbiosis appeared to be unaffected by menthol exposure, as demonstrated by successful reestablishment of the symbiosis when anemones were experimentally re-colonised with the homologous Symbiodinium (i.e. originating from the same host species). Furthermore, there was no significant impact on photosynthetic or respiratory performance of re-colonised anemones.

A novel application of statistically integrated transcriptomic and metabolomic analyses was applied in Chapter 3 to explore the molecular and metabolic pathways underlying symbiosisspecificity in the cnidarian-dinoflagellate symbiosis. Aposymbiotic individuals of the sea anemone Aiptasia generated by the methods optimised in Chapter 2 were colonised with either the homologous Symbiodinium type B1 or the novel Symbiodinium type D1a. RNA-seq gene expression analysis and gas chromatography-mass spectrometry-based metabolite profiling were conducted on the isolated host tissues. Analysis of the gene and metabolite expression 
profiles revealed that a novel symbiont confers an expression pattern intermediate between hosting a homologous symbiont, and having no symbiont. Although the formation and autotrophic potential of the novel association was similar to the homologous association, as determined by $\mathrm{O}_{2}$ flux measurements, the novel association resulted in an increase in the catabolism of metabolic stores (glycogen, lipid and protein), presumably in order to meet the energy requirements associated with the maintenance of cellular homeostasis. Integrated pathway analysis revealed reduced energy storage, an increased catabolism of stores, metabolic signalling and cellular redox homeostasis were molecular processes involved in the hosts' response to a novel symbiont type. This raised interesting questions as to how differences in the composition of symbiont-derived metabolites might feature in the specificity of the symbiosis.

Metabolite profiling was subsequently coupled with ${ }^{13} \mathrm{C}$-labelling in Chapter 4 , to identify the specific differences in the identity and quantity of photosynthetic carbon products translocated to the Aiptasia host when in symbiosis with the novel Symbiodinium type D1a versus the homologous Symbiodinium B1. A reduction in the diversity and quantity of net translocated products was observed in the novel association, despite achieving a similar autotrophic potential to the homologous association, as determined by $\mathrm{O}_{2}$ flux measurements. Interestingly, however, there was a continued fixation and translocation of carbon products to the host, suggesting that novel associations are, at least in part, metabolically functional in terms of photosynthate provision. Nevertheless, the decreased diversity and abundance of translocated products were associated with modifications to biosynthesis, increased catabolism of host energy stores, and oxidative stress-related signalling pathways in hosts colonised with the novel Symbiodinium type D1a.

The impact of symbiont type on the host's metabolite profile in an experimental setting raised the interesting possibility that the metabolite profiles of a cnidarian that forms natural flexible associations with different Symbiodinium types may also have dissimilar metabolite profiles, with implications for the nutritional physiology of the symbiosis. This was tested in Chapter 5. An in situ field survey of the metabolite profiles of Montipora capitata, a dominant reefbuilding coral in Hawai'i that forms homologous symbioses with Symbiodinium in clades C and clade D, revealed the metabolite pools of a coral host were not affected by Symbiodinium community composition. Given prior evidence that clade D can be less nutritionally beneficial to the coral host than some other symbiont types, the reasons for the similarity in metabolite profiles remains unclear. However, possible explanations are: 1) host heterotrophic compensation for the presence of a less beneficial symbiont; 2) modifications to the abundance 
and identity of metabolites exchanged in the symbiosis, perhaps via host selection of its symbiont community over time to provide an optimal state; or 3) adjustment to the host's metabolic pathway activity in response to different host-symbiont interactions, that produces similar free-metabolite profiles in the host. Nevertheless, this served to highlight that a coral host in a naturally flexible association, and under 'normal' environmental conditions, may maintain a steady metabolite profile irrespective of its Symbiodinium community composition. Notably, this contrasts with the findings that heterologous symbionts (i.e. those not usually associated with a particular host species) may have negative nutritional implications for the host that could ultimately restrict the success and persistence of novel host-symbiont pairings.

This study provides important evidence that optimal nutritional exchange and mechanisms of coping with oxidative stress in both partners are important determinants in the evolution of novel cnidarian-dinoflagellate symbioses. However, it also raises the possibility that such novel pairings, should they persist, may evolve over time to a more beneficial symbiotic state; this is worthy of further study. Indeed, we need to continue our efforts to understand the molecular and physiological mechanisms underpinning the adaptation of the cnidariandinoflagellate symbiosis to climate change, to facilitate the development of robust management strategies to safeguard the world's coral reefs. 


\section{ACKNOWLEDGEMENTS}

This project would not have been possible without the numerous scientists with whom I have been extremely blessed to be able to collaborate with and learn from over the past three years. First and foremost, I'd like to thank my supervisor Prof. Simon Davy, for his wisdom, support, and constructive feedback, and for providing me with this opportunity. I am extremely grateful for the extensive knowledge and valuable input provided by Prof. Virginia Weis and Prof. Arthur Grossman in the project. Credit must also be extended towards Dr Clint Oakley, for all the time and effort he invested in the project. I am also extremely grateful to the generosity of Prof. Ruth Gates at the Hawai'i Institute of Marine Biology, and Prof. Ute Roessner at Metabolomics Australia in Melbourne, for hosting me at their respective laboratories.

I am eternally grateful to all those who have sacrificed their time and provided crucial assistance in turning this ambitious project into a reality, especially Dr. Rob Keyzers, Dr. Elizabeth McKenzie, Dr. Erica Zarate, Dr. Siria Natera, Nirupama Samanmalie Jayasinghe, and Himasha Mendis for their indispensible metabolomics analysis training. Special thanks to Dr. Hollie Putnam, Dr. Ross Cunning, Raphael Ritson-Williams, and Chris Wall, for their assistance and advice during successful (and less successful) field trips to Hawai'i, and to Prof. Eli Meyer and Dr. Cammie Crowder for their RNA sequencing expertise. Special thanks to all the Davy Lab members, especially to Dr. Shaun Wilkinson for his wealth of knowledge in statistics, to Josh Brian for all his help with the anemones, and to Ashley Sproles, whom I am glad was by my side in this project, and through all the ups and downs of the last three years.

I would not be here if it wasn't for my second family at Big Blue Diving, Koh Tao, Thailand I intended to go for one week, but I stayed for four years. It was the experiences I had there that lead me on this path and ignited my passion for coral.

Everything I have achieved would not have been possible without the pervading support of my family, who allowed my to go confidently in the direction of my dreams and live the life I'd always imagined. I'd especially like to thank my Dad for his continuous encouragement and unwavering support; I am truly an arrow from his bow. And my beautiful Mum, who is the most generous and kind woman in the world, and whose parcels from England made Wellington feel a bit more like home. Thank you to my platinum sister Laura, who is still the only person to make me cry with laughter, and to Kate, for past and future adventures. And of course my puppy Jasper, just for the cuddles. Finally, thanks to my soon-to-be husband Darren, I will always be grateful for his patience and support, for being my best friend, for following me here and then listening to all my complaints, and for making life beautiful and exciting. 


\section{TABLE OF CONTENTS}

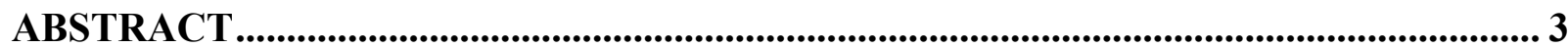

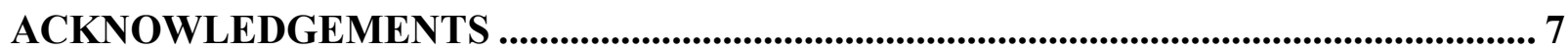

LIST OF FIGURES ............................................................................................................. 13

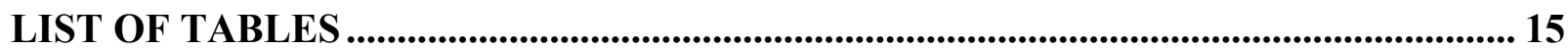

LIST OF ABBREVIATIONS ..................................................................................... 17

CONTRIBUTIONS AND PUBLICATIONS ........................................................................... 20

CHAPTER 1 General Introduction ............................................................................. 23

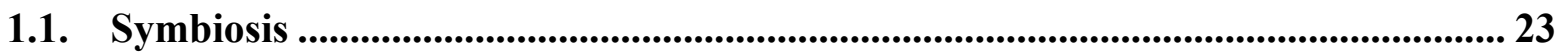

1.2. The cnidarian-dinoflagellate symbiosis ........................................................................ 24

1.2.1. Structure of the cnidarian-dinoflagellate symbiosis ........................................... 24

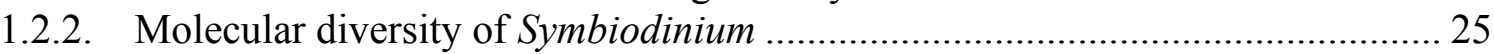

1.2.3. Physiological diversity of Symbiodinium ……………...................................... 27

1.3. Symbiont transmission, uptake and establishment .................................................. 28

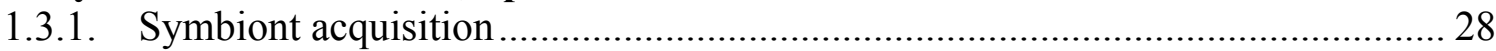

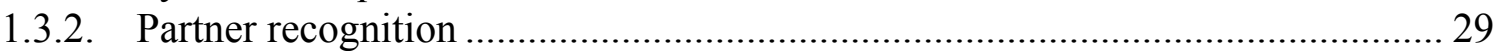

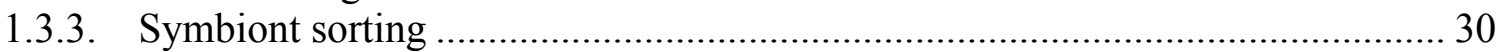

1.4. Specificity ........................................................................................................................... 32

1.5. Metabolic exchange.................................................................................................................. 34

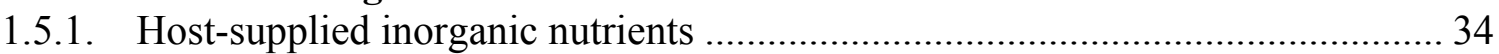

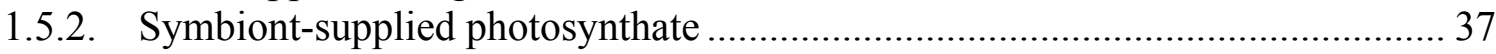

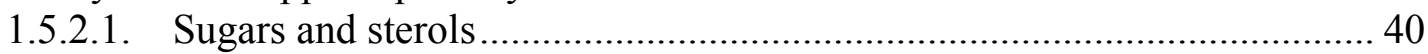

1.5.2.2. Amino acids and proteins ..................................................................... 40

1.5.2.3. Fatty acids and lipids .......................................................................... 41

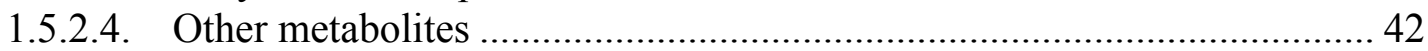

1.5.3. Quantity of translocated carbon-rich photosynthate …………………………. 42

1.5.4. Regulation and fate of metabolite translocation.................................................. 43

1.5.5. Taxonomic and environmental variation in metabolite profiles ......................... 44

1.6. The role of Symbiodinium in the response of corals to climate change................... 45

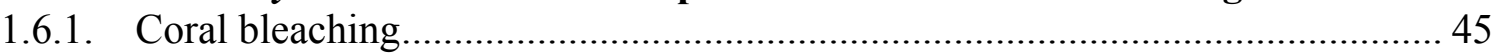

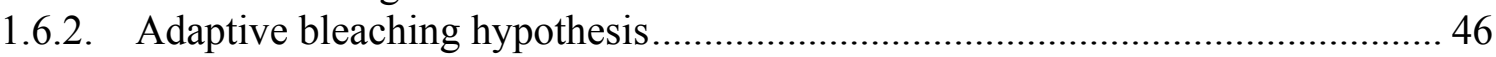

1.7. Gaps in our knowledge .................................................................................................... 48

1.8. The application of metabolomics........................................................................... 48

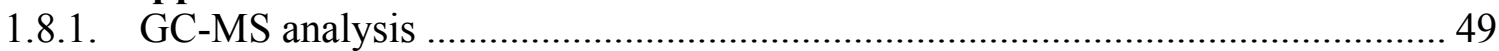

1.8.2. Application to the study of symbiont switching ................................................. 50

1.9. Aims and objectives ................................................................................................. 51

CHAPTER 2 Menthol-induced bleaching rapidly and effectively provides experimental aposymbiotic sea anemones (Aiptasia sp.) for symbiosis investigations.................................. 53

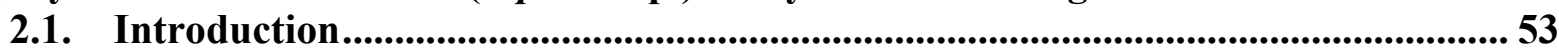

2.2. Materials and Methods....................................................................................5 54

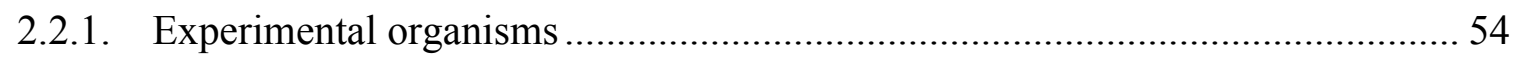

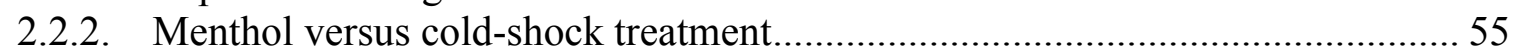

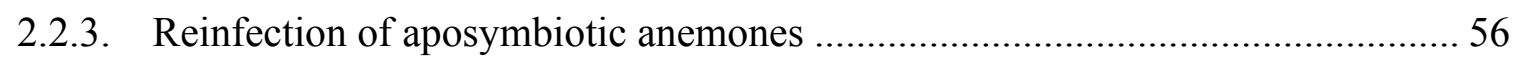

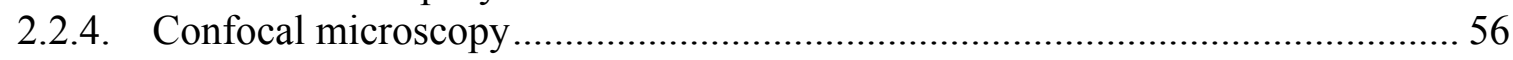

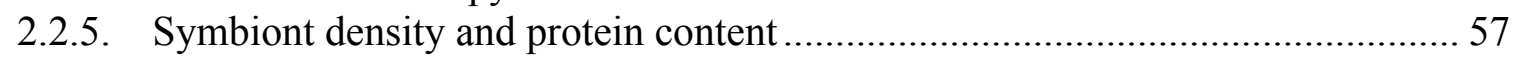

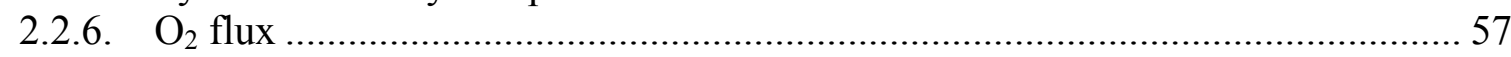

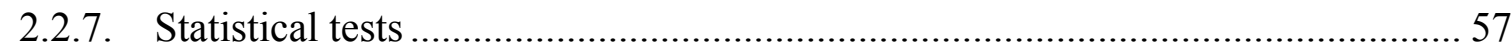




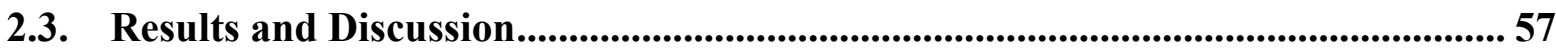

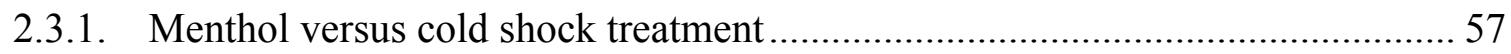

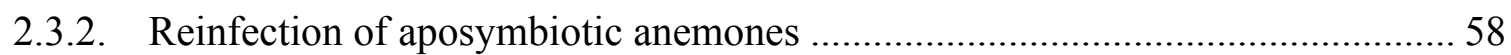

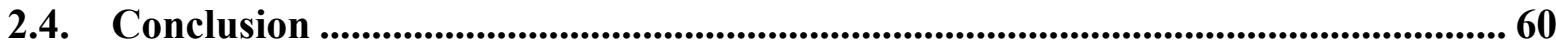

CHAPTER 3 Integrated pathway analysis of transcriptomic and metabolomics data suggests that optimal nutritional exchange and redox homeostasis operate in partner specificity in the cnidarian-dinoflagellate symbiosis ........................................................... 63

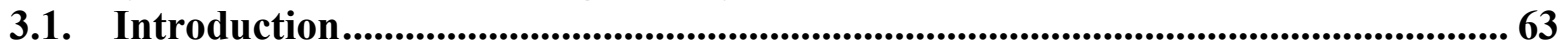

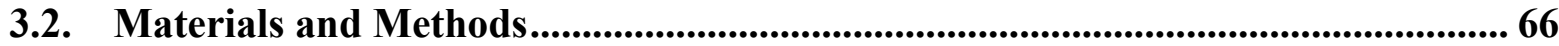

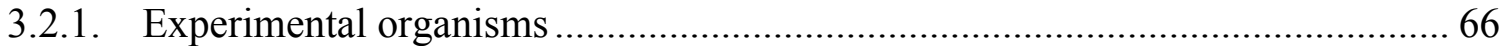

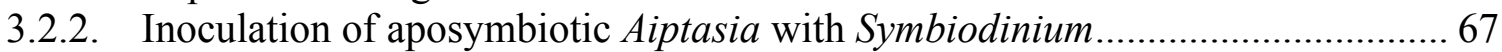

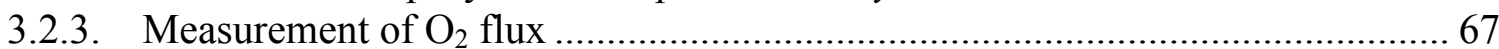

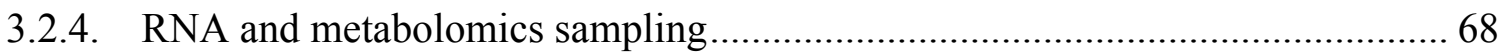

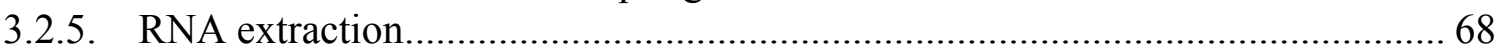

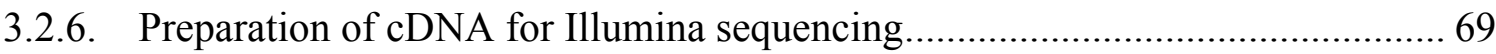

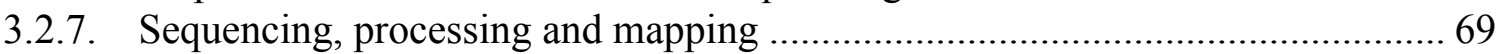

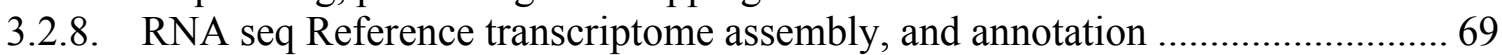

3.2.9. Identification of Differentially Expressed (DE) genes ......................................... 70

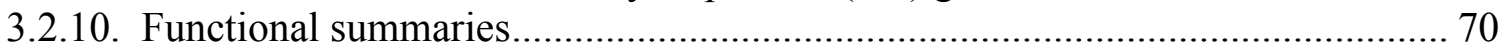

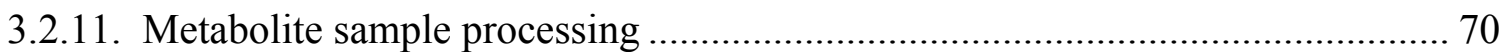

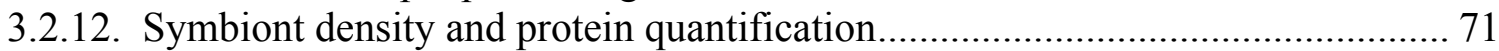

3.2.13. Symbiont community identification from host samples...................................... 71

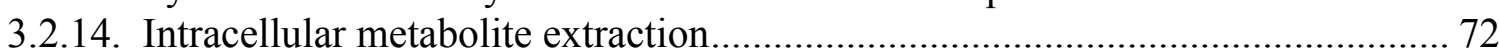

3.2.15. Metabolite data extraction, pre-processing and normalisation........................... 73

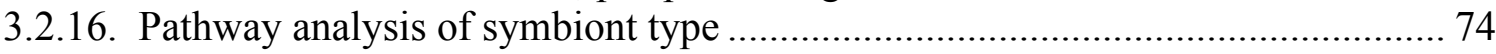

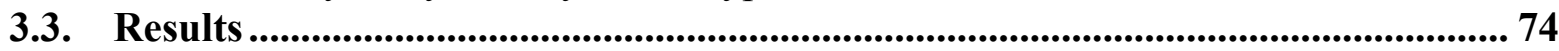

3.3.1. Symbiont type did not affect colonisation and autotrophic potential in

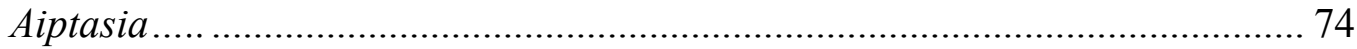

3.3.2. Symbiotic state affects a range of molecular processes in the cnidarian host..... 75

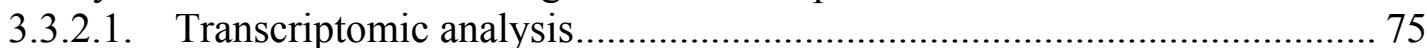

3.3.2.2. Annotation of differentially expressed transcripts .................................. 76

3.3.2.3. Metabolite profile comparison of the three symbiotic states ................... 79

3.3.3. Symbiont type affects host metabolite pools .................................................... 79

3.3.4. Symbiosis with a heterologous symbiont type induces increased catabolism of stored energy, metabolite signalling pathways and cellular redox responses .... 81

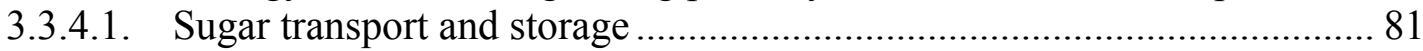

3.3.4.2. Lipid and protein metabolism and transport ........................................ 84

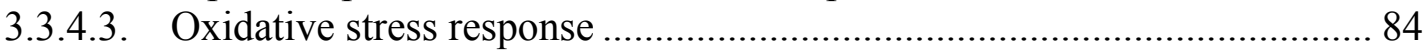

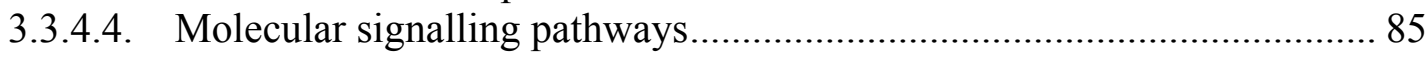

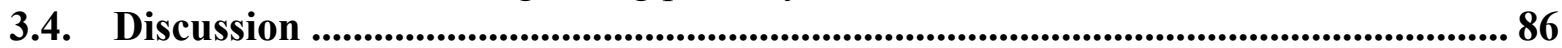

3.4.1. Heterologous symbiosis affects gene and metabolite expression profiles .......... 87

3.4.2. Evidence for the catabolism of host energy stores in a heterologous association 88

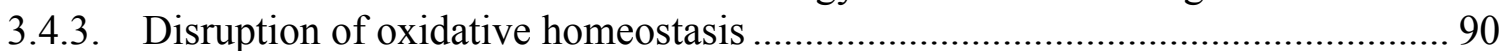

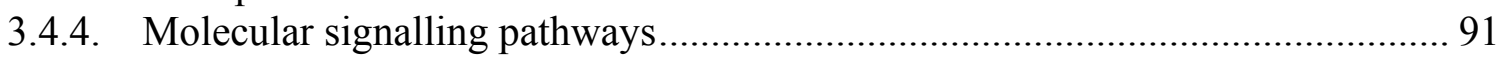

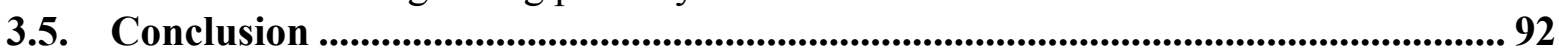

CHAPTER 4 Symbiont type affects the identity and quantity of organic carbon translocation and metabolism: Implications for partner switching in the cnidarian-

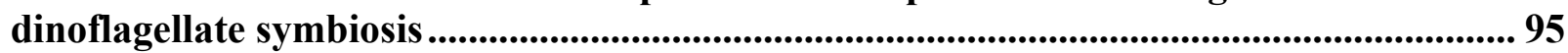

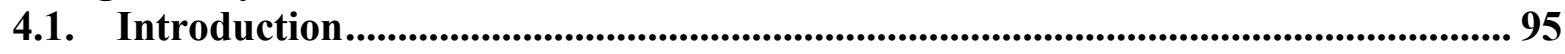

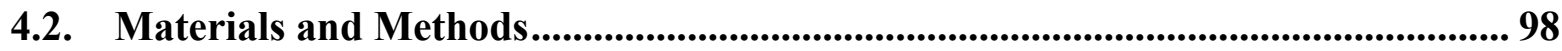

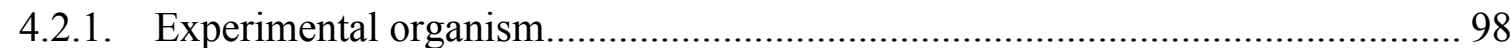




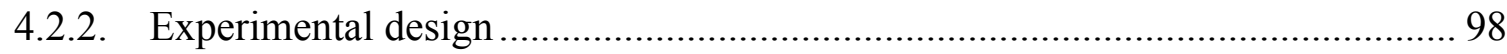

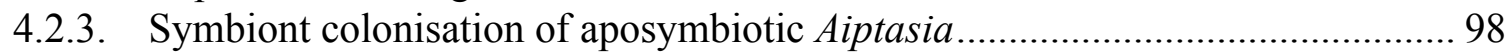

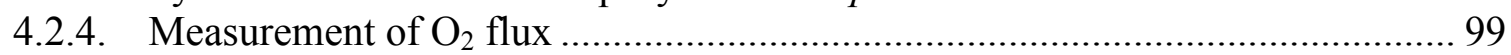

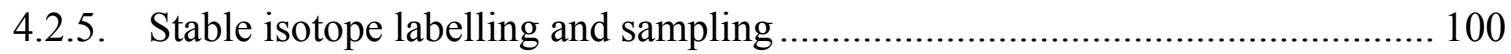

4.2.6. Metabolite profile sample host-symbiont separation ..................................... 100

4.2.7. Symbiont density and protein quantification................................................ 101

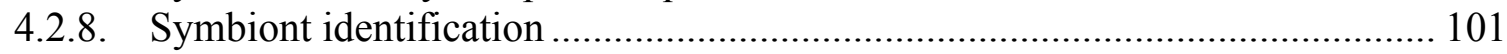

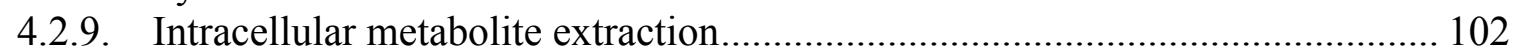

4.2.10. Metabolite data extraction, pre-processing and normalisation.......................... 103

4.2.11. Relative enriched metabolite abundance ......................................................... 103

4.2.12. Statistical analysis of relative enriched metabolite abundance .......................... 104

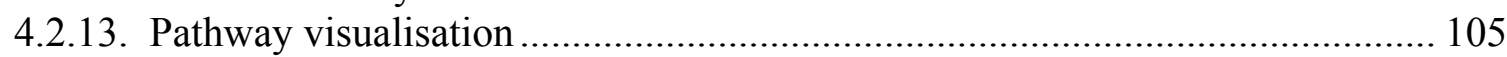

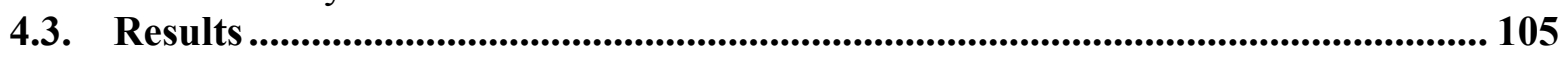

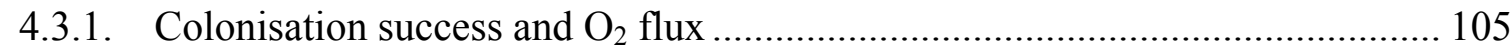

4.3.2. Alterations in host free metabolite pools induced by symbiotic state ............... 106

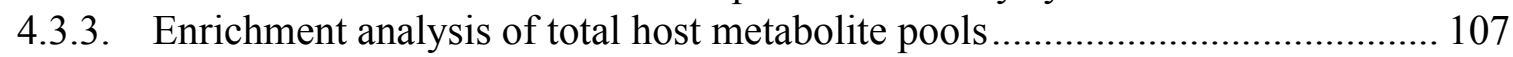

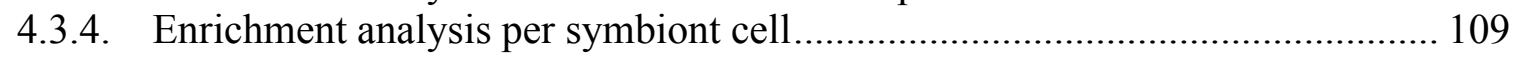

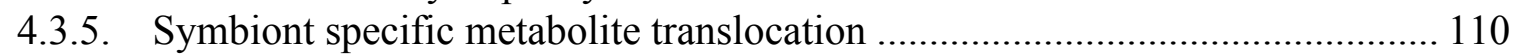

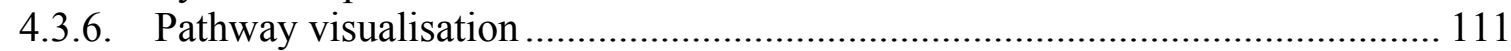

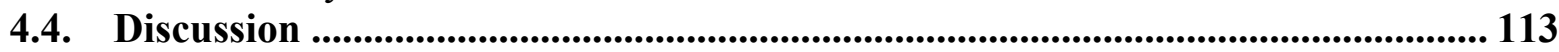

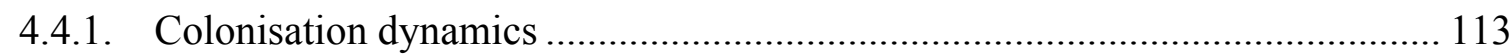

4.4.2. Symbiont type-specific modifications to glucose availability and lipogenesis. 114

4.4.3. Symbiont type effects on host fatty acid and lipid composition ....................... 116

4.4.4. Symbiont type-specific impacts on the host oxidative environment.................. 117

4.4.5. Evidence for the increased activity of the urea cycle in the heterologous

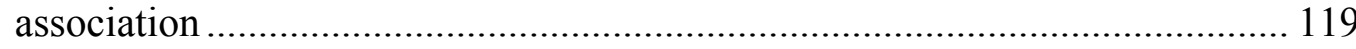

4.4.6. Inositol and maltose signalling for a successful symbiosis ............................ 120

4.5 Conclusion: Metabolic flux and the fate of the symbiosis .................................... 121

CHAPTER 5 Metabolite pools of the reef building coral Montipora capitata are unaffected by Symbiodinium community composition ........................................................... 123

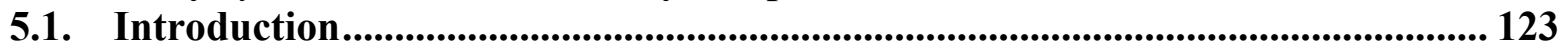

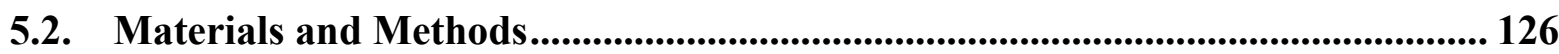

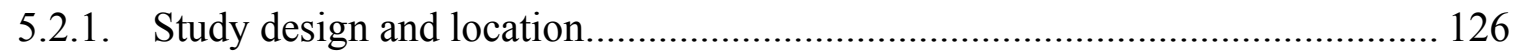

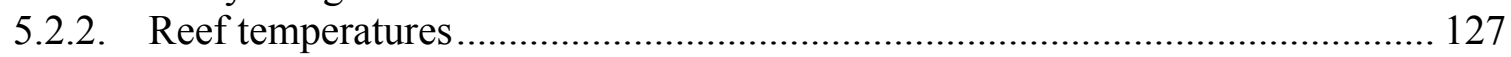

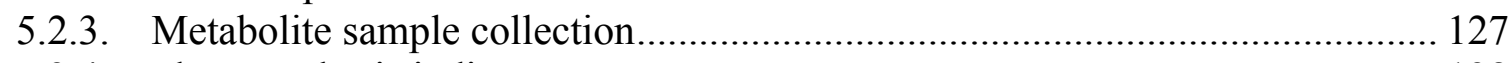

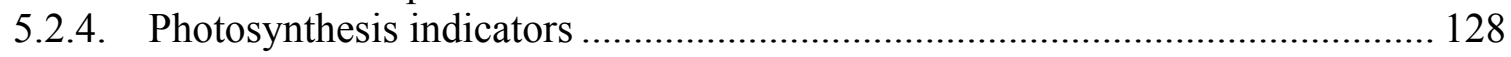

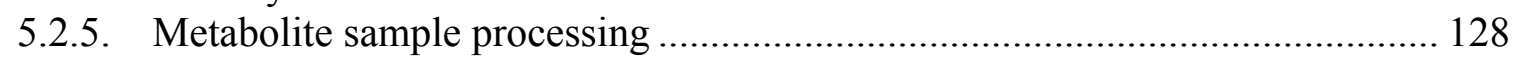

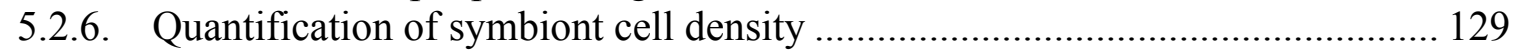

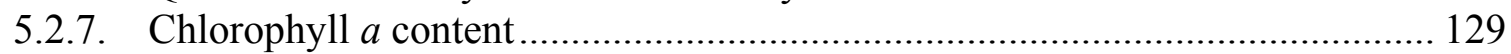

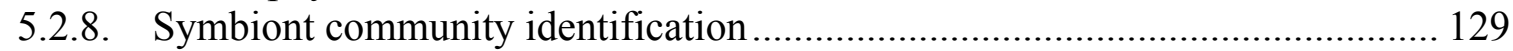

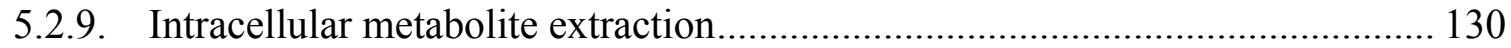

5.2.10. Metabolite data extraction, pre-processing, normalisation and statistical

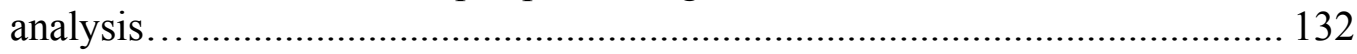

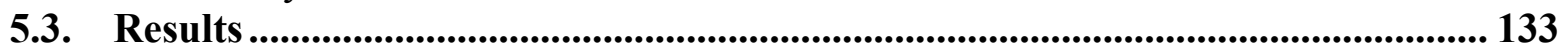

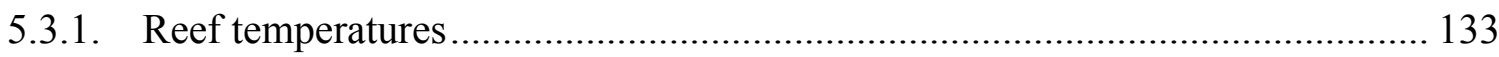

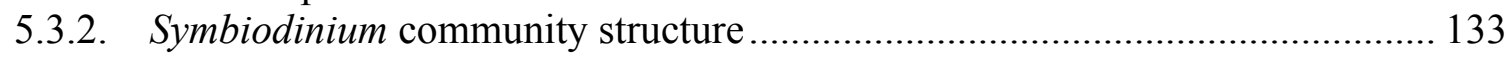

5.3.3. Photo-biological and symbiosis biomass parameters .................................... 135

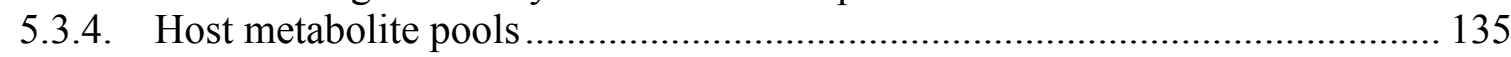

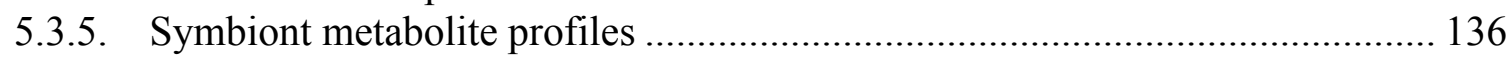

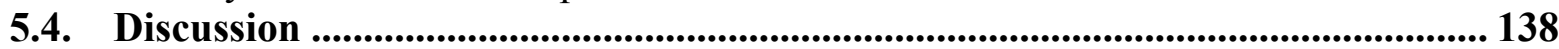


5.4.1. Symbiont community composition does not affect the metabolite profile of

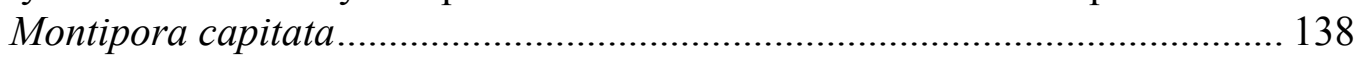

5.4.1.1. Host heterotrophic compensation ....................................................... 139

5.4.1.2. Modifications to the composition and quantity of translocated photosynthate by the symbionts and/or the host

5.4.1.3. Compensatory adjustments to metabolic pathway activity may generate similar metabolite profiles in the presence of different symbiont types. 142

5.4.2. Metabolic homeostasis as a requirement for the persistence of different, and potentially novel, host-symbiont pairings: the implications for holobiont adaptation

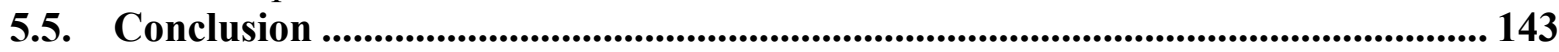

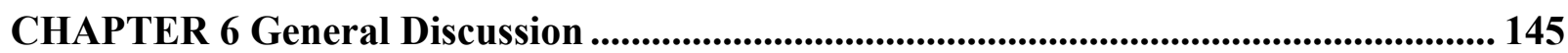

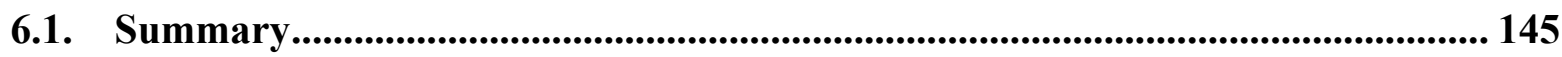

6.2. Mechanisms promoting flexibility .................................................................. 148

6.2.1. Will the formation of evolutionary novelties in the cnidarian-dinoflagellate symbiosis depend on the symbionts' antioxidant repertoire? ......................... 148

6.2.2. How do symbiont-derived metabolites feature in the molecular signalling pathways of the host's cells?

6.3. Implications for the survival of coral reefs ........................................................ 154

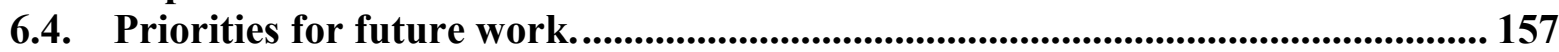

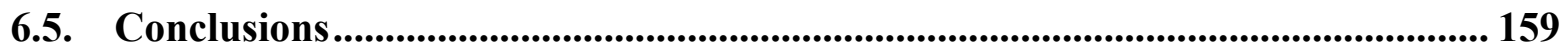

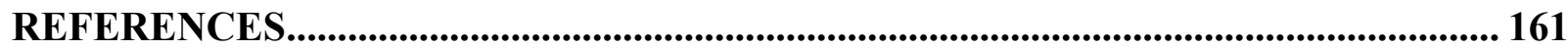

SUPPLEMENTAL FIGURES AND TABLES ........................................................ 184

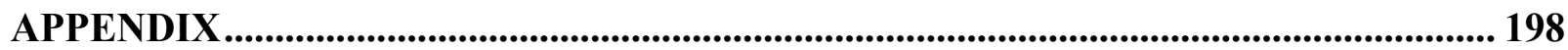

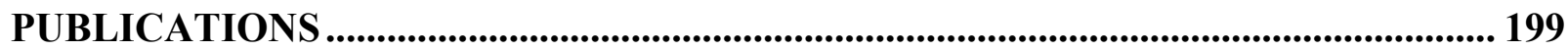




\section{LIST OF FIGURES}

Figure 1.1 Cross section showing histological organisation of a symbiotic cnidarian.... 24

Figure 1.2 Phylogenetic relationships between the major clades of Symbiodinium...... 26

Figure 1.3 The phases of symbiosis uptake and establishment in the cnidarianSymbiodinium symbiosis................................................ 32

Figure 1.4 Summary schematic of nutritional acquisition and exchange in the cnidariandinoflagellate symbiosis.......................................... 35

Figure 1.5 Coral bleaching.................................................... 45

Figure 1.6 Schematic of coral bleaching and symbiont shuffling and switching ........ 47

Figure 2.1 Effects of menthol and cold shock on algal cell density in Aiptasia sp........ 59

Figure 2.2 Repopulation of menthol-treated Aiptasia sp. with algal symbionts..........60

Figure 3.1 Aiptasia $\mathrm{sp}$. host gene expression in response to symbiotic state .............76

Figure 3.2 Host metabolite profile PCA scores plot, with $95 \%$ confidence intervals..... 79

Figure 3.3 Metabolic pathway visualisation of the fold-change in relative abundance of compounds detected in host free pools between heterologous and homologous associations

Figure 3.4 General overview of significantly affected pathways as indicated by differentially expressed genes and metabolites in the heterologous host tissues............................................................. 83

Figure 4.1 PCA scores plot of host metabolite profiles, with $95 \%$ confidence intervals......................................................... 106

Figure 4.2 Mean net relative abundance and enrichment of metabolites found in both homologous and heterologous host tissues............................ 109

Figure 4.3 Summary schematic of the enriched compounds detected in the host free pools in homologous and heterologous association........................... 112

Figure 4.4 Summary diagram of the metabolic flux and cellular consequences of harbouring different Symbiodinium types........................... 122

Figure 5.1 Study reef locations in Kāne'ohe Bay, O'ahu, Hawai'i, USA............... 127

Figure 5.2 Daily mean temperature from the reef locations for the two months prior to sample collection............................................... 133

Figure 5.3 Spatial patterns in Symbiodinium composition at each of the study reef locations in Kāne'ohe Bay, O'ahu, Hawai'i, USA....................... 134 
Figure 5.4 Host and symbiont metabolite profiles grouped according to Symbiodinium community composition, with $95 \%$ confidence intervals.................. 137

Figure 6.1 Conceptual diagram for the molecular mechanisms behind the establishment of novel cnidarian-dinoflagellate symbioses. .......................... 147

Figure 6.2 Potential inositol-signalling pathways............................... 154

Figure S5.1 Montipora capitata host and symbiont metabolite profiles grouped according to reef location................................................. 197 


\section{LIST OF TABLES}

Table 1.1 Example table of our current understanding for translocated compounds in the cnidarian-dinoflagellate symbiosis............................... 39

Table 3.1 Mean values for symbiont cell density and oxygen measurements of homologous type B1 and heterologous type D1a colonised Aiptasia....... 75

Table 3.2 Summary of the main molecular processes differentially expressed across the three symbiotic states (homologous, heterologous and aposymbiotic host

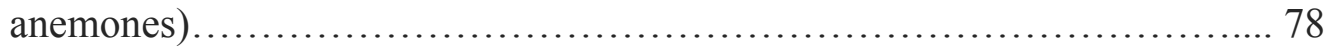

Table 3.3 A summary of the significantly enriched pathways (FDR $<0.05$ and at least 3 genes or metabolites present) per IMPaLa analysis of heterologous symbiontcontaining hosts against homologous symbiont-containing hosts........... 82

Table 4.1 Mean values for symbiont cell density and oxygen measurements of homologous type B1 and heterologous type D1a colonised Aiptasia........106

Table 4.2 Fold difference and percent label of the mean net metabolite abundance for the nine commonly labelled metabolites................................ 108

Table 4.3 Enriched compounds found in either homologous (green) or heterologous (blue) host tissues............................................. 111

Table 5.1 Quantitative PCR results for the Symbiodinium community composition of the M. capitata colonies collected.......................................134

Table 5.2 Photobiological characteristics of individual $M$. capitata colonies hosting different Symbiodinium community compositions .......................135

Table S3.1 Transcriptome assembly, mapping, and differential expression statistical values........................................................ 184

Table S3.2 Mean quantitative data of the metabolites identified in the host tissues of Aiptasia when aposymbiotic, or colonised with homologous (type B1), or heterologous (type D1a) Symbiodinium..................................185

Table S3.3 Fold change in relative metabolite abundance in the Aiptasia host tissues of heterologous D1a-colonised against a homologous B1-colonised (D1a/B1).... 187

Table S4.1 Summary of the mean comparative data for the metabolites identified in the host tissues of Aiptasia when aposymbiotic, or colonised with homologous (type B1), or heterologous (type D1a) Symbiodinium..... 188

Table S4.2 Summarised NTFD output for Aiptasia when aposymbiotic, or colonised with homologous (type B1), or heterologous (type D1a) Symbiodinium. 191 
Table S4.3 Summary of the test for significance between the net relative abundance of the unlabelled $\left(\mathrm{M}_{0}\right)$ and labelled $\left(\mathrm{M}_{\mathrm{i}}\right)$ fractions of the metabolites identified as enriched in both homologous B1-colonised and heterologous D1a-colonised Aiptasia host tissues

Table S4.4 Summary of the test for significance between the relative abundance of unlabelled $\left(\mathrm{M}_{0}\right)$ and labelled $\left(\mathrm{M}_{\mathrm{i}}\right)$ fractions per symbiont cell. 193

Table S5.1 Mean quantitative data of the metabolites identified in the host tissues of Montipora capitata grouped according to the Symbiodinium community composition. 194

Table S5.2 Mean quantitative data of the metabolites identified in the symbiont fraction of Montipora capitata grouped according to the Symbiodinium community composition. 196 


\section{LIST OF ABBREVIATIONS}

AMDIS Automated Mass Spectral Deconvolution and Identification System

ANOVA Analysis of variance

ARA

Arachidonic acid

BLAST Basic local alignment search tool

BSA

Bovine serum albumin

BSTFA N,O-bis-(trimethylsilyl)trifluoroacetamide

CA

Carbonic anhydrase

$\mathrm{CaCO}_{3} \quad$ Calcium carbonate

CCM Carbon concentrating mechanism

Chl Chlorophyll

$\mathrm{CO}_{2} \quad$ Carbon dioxide

CoA Coenzyme A

CTAB Cetyltrimethylammonium bromide

DCMU 3-(3,4-dichlorophenyl)-1,1-dimethylurea

DE Differentially expressed

DIC Dissolved inorganic carbon

DIN Dissolved inorganic nitrogen

DIP Dissolved inorganic phosphorus

DMF N, N-dimethylformamide

DNA Deoxyribonucleic acid

DOM Dissolved organic material

DOP Dissolved organic phosphorus

EDTA Ethylenediaminetetraacetic acid

EI Electron ionization

FC Fold change

Fd Ferredoxins

FDR False discovery rate

FSW Filtered seawater

$\mathbf{F}_{\mathbf{v}} / \mathbf{F}_{\mathbf{m}} \quad$ Maximum dark-acclimated yield of photosystem II

GCLC Glutamate-cysteine ligase catalytic subunit

GC-MS Gas chromatography - mass spectrometry

GDH Glutamate dehydrogenase

GIn Glutamine

Glu Glutamate 


\begin{tabular}{|c|c|}
\hline GO & Gene ontology \\
\hline GOGAT & Glutamine:2-oxoglutarate aminotransferase \\
\hline GPCR & G-protein coupled receptor \\
\hline GPI & Glycosylphosphatidylinositol \\
\hline GS & Glutamine synthetase \\
\hline GSH & Glutathione \\
\hline Gst & Glutathione S-transferase \\
\hline $\mathbf{H}_{2} \mathbf{O}_{2}$ & Hydrogen peroxide \\
\hline $\mathrm{HCO}_{3}^{-}$ & Bicarbonate \\
\hline HRF & Host release factor \\
\hline IMPaLA & Integrated molecular pathway level analysis \\
\hline IS & Internal standard \\
\hline ITS & Internal transcribed spacer \\
\hline KEGG & Kyoto Encyclopedia of Genes and Genomes \\
\hline LC-MS & Liquid chromatography-mass spectrometry \\
\hline MAA & Mycosporine-like amino acid \\
\hline MALDI-MSI & Matrix-assisted laser desorption ionization mass spectrometry imaging \\
\hline MD & Metabolite Detector \\
\hline MID & Mass isotopomer distribution \\
\hline MUFA & Monounsaturated fatty acid \\
\hline $\mathbf{m} / \mathbf{z}$ & Mass-to-charge ratio \\
\hline NADPH & Nicotinamide adenine dinucleotide phosphate \\
\hline nanoSIMS & Nanoscale secondary ion mass spectrometry \\
\hline NO & Nitric oxide \\
\hline $\mathrm{NO}_{2}^{-}$ & Nitrite \\
\hline $\mathrm{NO}_{3}^{-}$ & Nitrate \\
\hline $\mathbf{N H}_{4}^{+}$ & Ammonium \\
\hline NMR & Nuclear magnetic resonance \\
\hline NTFD & Non-Targeted Tracer Fate Detection \\
\hline $\mathbf{O}_{2}^{-}$ & Superoxide ion \\
\hline PAM & Pulse amplitude modulation \\
\hline $\mathbf{P C}$ & Principal component \\
\hline PCA & Principal components analysis \\
\hline PCR & Polymerase chain reaction \\
\hline $\mathbf{P}_{\text {gross }}$ & Gross photosynthesis \\
\hline $\mathbf{P}_{\text {net }}$ & Net photosynthetic oxygen evolution \\
\hline
\end{tabular}




\begin{tabular}{ll} 
POM & Particulate organic material \\
POP & Particulate organic phosphate \\
PUFA & Polyunsaturated fatty acid \\
qPCR & Quantitative polymerase chain reaction \\
R & Respiration \\
RI & Retention index \\
RNA & Ribonucleic acid \\
RNA-Seq & Next generation RNA sequencing \\
RNS & Reactive nitrogen species \\
RONS & Reactive oxygen and nitrogen species \\
ROS & Reactive oxygen species \\
RT & Retention time \\
SAM & Significance analysis of microarrays (and metabolites) \\
SDS & Sodium dodecyl sulphate \\
S.E.M. & Standard error mean \\
SLC & Solute carrier \\
SOD & Superoxide dismutase \\
SSU & Small subunit \\
TAG & Triacylglycerol \\
TCA & Tricarboxylic acid \\
TMS & Trimethylsilyl \\
TRP & Transient receptor potential \\
\hline
\end{tabular}




\section{CONTRIBUTIONS AND PUBLICATIONS}

This thesis is written as a series of manuscripts either already published, or in preparation for submission in the near future. As such, there may be some repetition of themes between chapters (particularly in "Introduction" sections). Tables and figures that were too large for the printed document are available on the Appendix CD provided. This thesis is the intellectual and analytical work of the author, with assistance as described below.

Chapter 2: This chapter is formatted as a stand-alone manuscript based on a recently published study:

Matthews J.L., Sproles A.E., Oakley C.A., Grossman A.R., Weis V.M., Davy S.K. (2016) Menthol-induced bleaching rapidly and effectively provides experimental aposymbiotic sea anemones (Aiptasia sp.) for symbiosis investigations. Journal of Experimental Biology 219:306-310

A.E. Sproles assisted with sample processing. Confocal microscopy was conducted the School of Biological Sciences, VUW. A.E. Sproles, C.A. Oakley, A.R. Grossman, V.M. Weis and S.K. Davy advised on the experimental design and manuscript preparation. The published paper is provided at the end of the manuscript.

Chapter 3: This chapter is formatted as an independent manuscript:

Matthews J.L., Crowder C.M., Oakley C.A., Lutz A., Roessner U., Meyer E., Grossman A.R., Weis V.M., and Davy S.K. Integrated pathway analysis of transcriptomic and metabolomic data suggests that optimal nutritional exchange and redox homeostasis operate in partner specificity in the cnidarian-dinoflagellate symbiosis.

R.A. Keyzers assisted with GC-MS analysis of preliminary samples at the School of Chemistry and Physical Sciences, Victoria University of Wellington. RNA sequencing preparation and analysis was conducted at the Department of Integrative Biology, Oregon State University, Oregon, USA. C.M. Crowder and E. Meyer assisted with RNA sample processing and differential expression analysis. GC-MS analysis was undertaken at Metabolomics Australia, The University of Melbourne, Australia. A. Lutz, N. Jayasinghe, and H. Mendis assisted with sample preparation and GC-MS analysis. C.A. Oakley, A.R. Grossman, V.M. Weis, U. Roessner and S.K. Davy advised on the experimental design, data analysis, interpretation, and manuscript preparation. 
Chapter 4: This chapter is formatted as an independent manuscript:

Matthews J.L., Hillyer K.E., Oakley C.A., Lutz A., Roessner U., Grossman A.R., Weis V.M., and Davy S.K. Symbiont type affects the identity and quantity of organic carbon translocation and metabolism: Implications for partner switching in the cnidarian-dinoflagellate symbiosis.

GC-MS analysis was undertaken at Metabolomics Australia, The University of Melbourne, Australia. A. Lutz, N. Jayasinghe, and H. Mendis assisted with sample preparation and GCMS analysis. K.E. Hillyer, C.A. Oakley, A.R. Grossman, V.M. Weis, U. Roessner and S.K. Davy advised on the experimental design, data analysis, interpretation, and manuscript preparation.

Chapter 5: This chapter is formatted as an independent manuscript:

Matthews J.L., Ritson-Williams R., Cunning R., Oakley C.A., Lutz A., Roessner U., Grossman A.R., Weis V.M., Gates R.D. and Davy S.K. Metabolite pools of the reef building coral Montipora capitata are unaffected by Symbiodinium community composition.

Sample collection was conducted at the Hawai'i Institute of Marine Biology, Kāne'ohe Bay, Hawai'i, USA. R. Ristson-Williams and R. Cunning assisted with sample collections. GC-MS analysis was undertaken at Metabolomics Australia, The University of Melbourne, Australia. A. Lutz, N. Jayasinghe, and H. Mendis assisted with sample preparation and GC-MS analysis. C.A. Oakley, U. Roessner, A.R. Grossman, V.M. Weis, R.D. Gates and S.K. Davy advised on the experimental design, data analysis, interpretation, and manuscript preparation. 


\section{CHAPTER 1}

\section{General Introduction}

\subsection{Symbiosis}

Symbiosis, as defined by mycologist Anton de Bary (1879), describes a relationship between dissimilar species living intimately together for prolonged periods. Symbiotic interactions have played a fundamental role in shaping life on earth. These associations are considered to be either ectosymbiotic, where a symbiotic partner remains outside the other (e.g. cleaner fish), or endosymbiotic, where the symbiont (the smaller symbiotic partner) lives within the tissues of the larger host organism (e.g. bacteria in the rumen of ungulates, or symbiotic algae within coral tissues). Endosymbiotic associations exist across all ecosystems and are considered a driving force for evolution (Margulis 1973). Endosymbiotic theory proposes that organelles, such as mitochondria and chloroplasts, were once prokaryotic cells that escaped digestion by the larger host cell (Whatley et al. 1979). The evolution of such endosymbioses was perhaps a result of the provision of crucial nutrients by the invading cells, as was the case for the primitive chloroplast (Whatley and Whatley 1981).

The terms parasitic, commensal, or mutual are used to further categorise symbiotic associations (Smith 1993), though in reality the relationships between species are far more complex and are better viewed as a graduation between the three categories (Smith and Douglas 1987; Douglas 1994). Parasitism describes the circumstances in which one partner will benefit at the expense of the other, while a symbiosis in which one partner benefits with no influence on the other is known as a commensal relationship. Mutualism, however, provides fitness benefits for both partners, and often involves the ability of at least one partner to obtain a novel metabolic capability, such as nitrogen fixation in legumes (Herre et al. 1999; Moné et al. 2014). This relationship is not always equally beneficial to both partners. In particular, facultative mutualists can survive and/or reproduce in isolation of the symbiotic partner, e.g. aphids and their facultative bacterial endosymbionts (Koga et al. 2003), whereas an obligate mutualist cannot survive for long periods in isolation, often as a result of an underlying physiological constraint, e.g. symbiotic algae in the tissues of reef building corals (Muscatine and Hand 1958; Muscatine and Cernichiari 1969; Cook 1985; Muscatine 1990). 


\subsection{The cnidarian-dinoflagellate symbiosis}

Symbiotic associations between animals and photosynthetic microorganisms are prevalent across many marine invertebrate phyla, and are particularly common in modern coral reefs (Trench 1979; Vacelet 1981). However, perhaps the best-known example is the obligate endosymbiosis between Cnidaria (including corals and sea anemones, jellyfish, and hydrozoans) and unicellular algal dinoflagellates of the genus Symbiodinium (Freudenthal 1962; Trench 1987). Palaeolithic evidence suggests the origin of this photosymbiosis in reef building corals was some 200 millions years ago, during the Triassic period (Muscatine et al. 2005; Stanley Jr 2006). Currently, coral reefs account for less than $0.1 \%$ of the Earth's surface, occupying a narrow band around the equator to latitudes around $30^{\circ} \mathrm{N}$ to $30^{\circ} \mathrm{S}$ (Muller-Parker and Davy 2001). Nevertheless, coral reef ecosystems are highly diverse and incredibly efficient, providing homes and nurseries to approximately $25 \%$ of marine species (Burke et al. 2002. Moreover, coral reefs act as an important source of protein, income and coastal protection for surrounding coastal communities (Moberg and Folke 1999).

\subsubsection{Structure of the cnidarian-dinoflagellate symbiosis}

Within cnidarians, Symbiodinium are typically located within the cell cytoplasm of the host's gastrodermis (Figure 1.1). The term "symbiosome" describes the intracellular symbiotic compartment in which the symbiont resides and its surrounding host-derived membrane (the "symbiosome membrane"), internally lined with multiple layers of dinoflagellate-derived membranes (Roth et al. 1988; Wakefield and Kempf 2001). Together these membranes form the "symbiosome membrane complex".

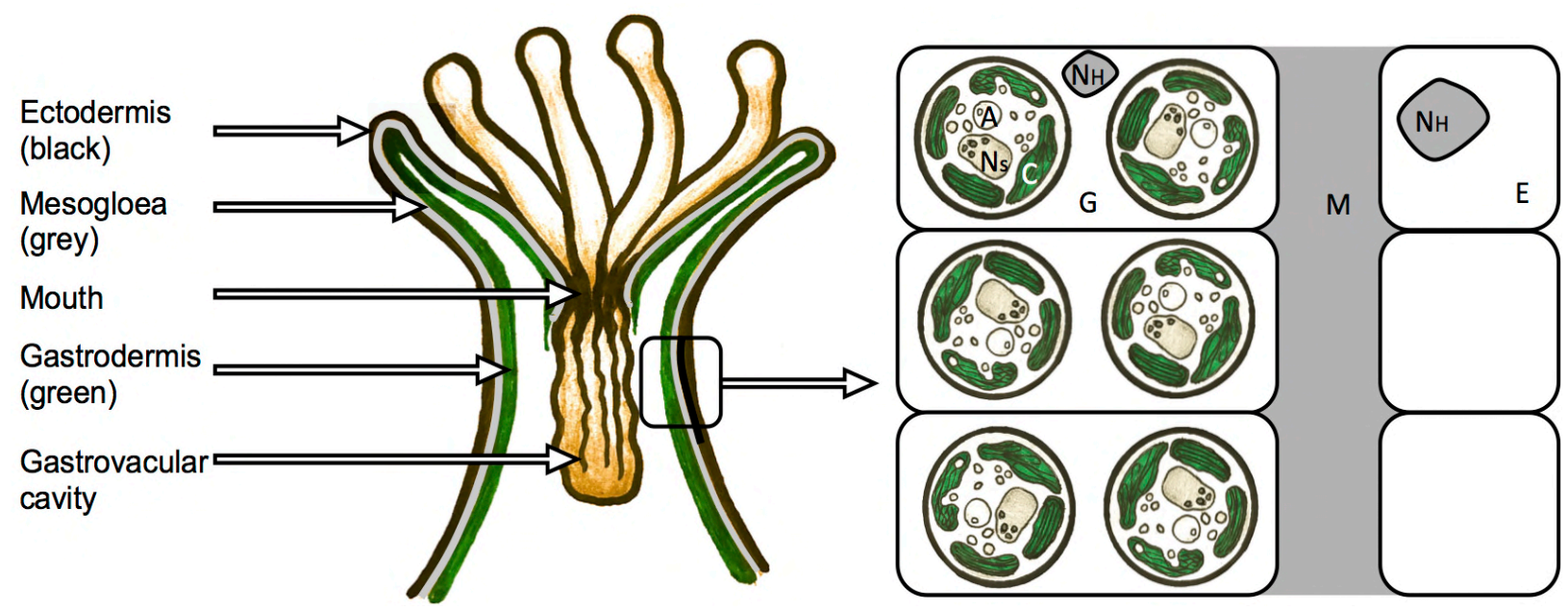

Figure 1.1 Cross section showing histological organisation of a symbiotic cnidarian. The close-up view of the three body layers shows the ectodermis (E) (host nucleus $\mathrm{N}_{\mathrm{H}}$ ), mesogloea $(\mathrm{M})$, and gastrodermis $(\mathrm{G})$ within which the Symbiodinium (represented as green structures in gastrodermis) are found in symbiosomes (chloroplast $\mathrm{C}$, accumulation body $\mathrm{A}$, and symbiont nucleus $\mathrm{N}_{\mathrm{S}}$; modified from Gates et al. 1992; Allemand et al. 2011). 


\subsubsection{Molecular diversity of Symbiodinium}

The genus Symbiodinium encompasses the largest and most prevalent group of endosymbiotic dinoflagellates. The dinoflagellates occupy two major stages in their life history: the motile (dinomastigote) and non-motile (coccoid) stages. Each Symbiodinium cell is coccoid in hospite, and these spherical cells are 6 to $13 \mu \mathrm{m}$ in diameter on average, depending on the species (Blank and Huss 1989). Asexual propagation (mitosis) occurs exclusively in the coccoid state (Fitt and Trench 1981), however population genetic evidence supports the view that Symbiodinium cells periodically undergo sexual recombination (Baillie et al. 2000; LaJeunesse 2001; Santos et al. 2004).

Although previously thought to be a single pandemic species known as Symbiodinium microadriaticum (Freudenthal 1962; Taylor 1974), molecular phylogenetic evidence over the last two decades has shown there to be great diversity in the genus Symbiodinium, of which numerous clades and sub-clades ('types') are now known to exist (Baker et al. 2004; Coffroth and Santos 2005; LaJeunesse et al. 2012). Sequencing of ribosomal small sub-unit (SSU) and mitochondrial cytochrome oxidase 1 (CO1) genes provisionally identified large genetic disparity between clades in the genus Symbiodinium compared to other dinoflagellates (Rowan and Powers 1992; LaJeunesse 2001; Stern et al. 2010). Currently, variable sequence data from the nuclear internal transcribed spacer unit 2 (ITS2) provides a reasonable method of determining types (Thornhill et al. 2007; Sampayo et al. 2009; LaJeunesse and Thornhill 2011). However, the use of multiple genetic markers from different organelles e.g., mitochondrial CO1, nuclear ITS2, and chloroplast 23S (cp23s), along with hierarchical phylogenetic classification provides the genetic resolution necessary for investigating species diversity, biogeography, dispersal, natural selection and adaptive radiations (Sampayo et al. 2009; LaJeunesse et al. 2014). Furthermore, multilocus microsatellite genotyping and the application of next-generation sequencing methods will help to further clarify the resolution of species boundaries and refine the diversification, distribution and gene flow of Symbiodinium, and help resolve the distinction of low-abundance background symbionts (Coffroth and Santos 2005; Pettay et al. 2011; Stat et al. 2012; Thornhill et al. 2014; Boulotte et al. 2016; Wham and LaJeunesse 2016).

At present, nine Symbiodinium clades are described (A-I) (Stat and Gates 2011), of which six (A-D, F-G) associate with reef-building corals (Figure 1.2) (Baker 2003; LaJeunesse et al. 2004; Hunter et al. 2007; van Oppen et al. 2009). In terms of species, ITS2 types currently number in the hundreds, but many communities of symbiotic cnidarian around the world still 
require sampling, in addition to resolving the species identity of those free-living and foraminifera-associating Symbiodinium (Finney et al. 2010; LaJeunesse and Thornhill 2011; Pettay et al. 2011; Aranda et al. 2012; Arif et al. 2014; Wham and LaJeunesse 2016). The nomenclature for the genus Symbiodinium involves capital letters to indicate clade designation, while types are described using an alphanumerical system (e.g., A1, A2, A3 etc.). The ITS region is a fast evolving area, and therefore some types contain more than one co-dominant repeat within their genome; these co-dominant intragenomic ITS variants are further identified by designation of a lowercase letter $(\mathrm{Cla}, \mathrm{C} 1 \mathrm{~b}, \mathrm{C} 1 \mathrm{c}$ etc., continuing onto a double letter after the number of types exceed the number of letters in the alphabet, e.g., C1aa, C1bb etc.) (LaJeunesse 2005). However, due to recent evidence that similar sequences occurred as codominant repeats across various types in clade D, there is now advocacy for the use of Symbiodinium species names instead e.g. type D1a is now commonly referred to as Symbiodinium trenchii (LaJeunesse et al. 2010a; LaJeunesse et al. 2010b). Currently there are 20 named species of Symbiodinium, but half are in need of proper species descriptions (LaJeunesse et al. 2012).

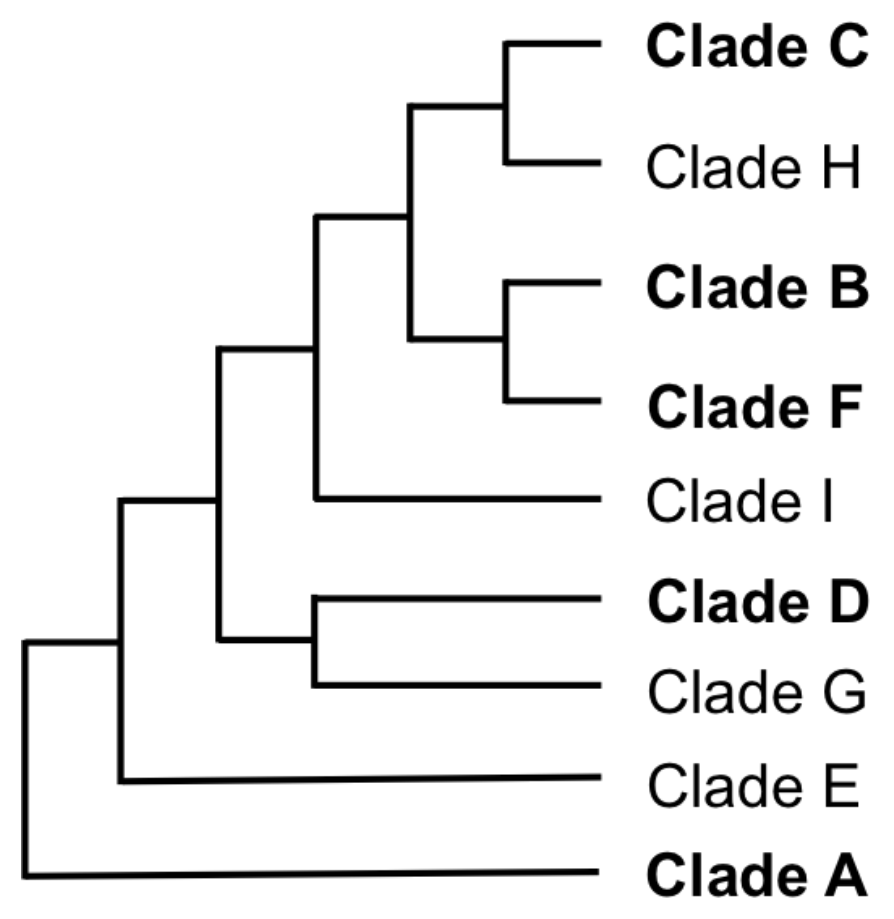

Figure 1.2 Phylogenetic relationships between the major clades of Symbiodinium. Taxa within each clade of Symbiodinium exhibit genetic diversity. Bold font represents those clades associated with scleractinian corals. 


\subsubsection{Physiological diversity of Symbiodinium}

Given the large diversity of Symbiodinium revealed by molecular analysis, it is not surprising that significant physiological diversity exists at both the clade and type level, and among populations of the same types that exist in different environmental settings (Howells et al. 2012; Hume et al. 2013; Parkinson et al 2015). This includes a variation between Symbiodinium types in terms of photosystem function and repair (Sampayo et al. 2008a; LaJeunesse et al. 2010b), and thermal resistance to prevent coral bleaching (Buddemeier and Fautin 1993; Iglesias-Prieto and Trench 1997; Rowan 2004; Tchernov et al. 2004; Loram et al. 2007; Sampayo et al. 2008a; Stat et al. 2008). For example, photophysiological differences in Symbiodinium have been attributed to the differential damage and subsequent repair of the D1 protein, a protein component of photosystem II (Warner et al. 1999). Similarly, there are variations between Symbiodinium types in the quantity and quality of UV light-absorbing mycosporine amino acids (MAA), which act to prevent photodamage (Banaszak et al. 2000; Reynolds et al. 2008). For instance, the MAA composition of clade A Symbiodinium has been hypothesised to explain their ability to associate with corals in shallow-water, high-light environments (Reynolds et al. 2008). A range of other traits related to thermotolerance have also been shown to differ between Symbiodinium types, including photokinetics (Jones and Berkelmans 2012), photosystem gene expression (McGinley et al. 2012), expression of coral putative stress-response genes (Yuyama et al. 2012), nitric oxide synthesis (Hawkins and Davy 2012), production of reactive oxygen species and antioxidants (McGinley et al. 2012; Krueger et al. 2014; Krueger et al. 2015), and lipid composition (Díaz-Almeyda et al. 2011; Jones and Berkelmans 2011). Furthermore, distinct Symbiodinium types are affected differently by ocean acidification, at least in vitro (Brading et al. 2011) and they also differ in intracellular $\mathrm{pH}$ (Gibbin and Davy 2013). Consequently, the same host can display different physiologies in response to the Symbiodinium type(s) it hosts (Rowan 2004; Goulet et al. 2005), the ramifications of which will be discussed below (see Section 1.6) (Buddemeier and Fautin 1993; Little et al. 2004; Rodriguez-Lanetty et al. 2004).

While metabolic exchange in the symbiosis is described in greater detail below (see Section 1.5), it is important to note here that there are different modes of inorganic carbon acquisition amongst Symbiodinium (Brading et al. 2013), as well as differences in the amount of carbon and nitrogen translocated to the coral host tissues (Stat et al. 2008; Cantin et al. 2009; Baker et al. 2013; Starzak et al. 2014; Pernice et al. 2015). Also, carbon translocation from symbiont to host can depend on irradiances and planktonic food availability (Tremblay et al. 2014), while 
nitrate composition can also change within a coral-dinoflagellate association in response to different temperatures (Baker et al. 2013).

Furthermore, there are ecological differences among Symbiodinium including motility and infectivity (Schoenberg and Trench 1980a; Fitt and Trench 1981; Bay et al. 2011), and temperature has been shown to moderate the infectiousness of conspecific Symbiodinium from the same host population (Hawkins et al. 2016a). Infectivity of a symbiont is a useful phenotype to assess, as it is the first stage in the establishment of the symbiosis.

\subsection{Symbiont transmission, uptake and establishment}

Since Symbiodinium are closely related to parasitic ampicomplexans - important animal and human pathogens - much might be learned from comparing their colonisation dynamics, in terms of understanding the capacities, weaknesses and evolution of ampicomplexans (Shoguchi et al. 2013b).

\subsubsection{Symbiont acquisition}

Symbionts are either transferred from parent to offspring via direct inheritance, known as 'vertical transmission', or acquired anew with each generation from the surrounding environment, known as 'horizontal transmission' (Rowan and Knowlton 1995; Weis et al. 2001; Gómez-Cabrera et al. 2008).

Horizontal transmission occurs in $\sim 85 \%$ of symbiotic corals, indicating the presence of evolutionary constraints that limit vertical transmission, or higher ecological costs associated with vertical transmission (Yakovleva et al. 2009). For example, with vertical symbiont transmission, host progeny benefit by not having to search for their symbionts, and this acts to maintain advantageous symbionts (Padilla-Gamino et al. 2012; Byler et al. 2013). However, host progeny encountering an environment that differs from that of their parent may be disadvantaged as a result of hosting a suboptimal symbiont (Weis et al. 2001). Horizontal transmission, in comparison, involves the release of aposymbiotic (dinoflagellate-free) progeny, which allows for the acquisition of novel, potentially advantageous symbionts during the larval (planula) and/or juvenile stages. This mechanism is particularly beneficial following long-distance dispersal of planulae into new environments (LaJeunesse et al. 2004; Baird et al. 2007). Indeed, larvae or juveniles of most coral species are sometimes able to initially associate with either homologous (same as adult colony) or heterologous types of 
Symbiodinium (Schwarz et al. 1999; Abrego et al. 2009a; Cantin et al. 2009; Harii et al. 2009), as also reported for cultured gorgonians (Coffroth et al. 2001) and sea anemones (Schoenberg and Trench 1980a; Davy et al. 1997a). Subsequently, the initial distribution of symbionts can shift during host colony development to reflect that found in adjacent adult corals, highlighting the presence of specific mechanisms that ultimately favours the development of an optimal relationship between the coral and the symbiont (Coffroth et al. 2001; Gómez-Cabrera et al. 2008; Abrego et al. 2009a).

Strong partner preference and symbiont sexual recombination can, in theory, act in order to allow novel mutualisms to persist and evolve in systems with horizontal transmission, although the ability of corals to maintain these novel associations through to adulthood is largely unknown (but see Section 1.4) (Gómez-Cabrera et al. 2008; Abrego et al. 2009b,a; Byler et al. 2013; Cumbo et al. 2013). Furthermore, as horizontal transmission does not ensure the perpetuation of novel associations for subsequent generations, and as aposymbiotic larvae may or may not acquire an advantageous symbiont, each generation is left to gamble with the continuation of a beneficial symbiosis independent of the parent's association (Weis et al. 2001; Byler et al. 2013). The distribution, abundance and persistence of free-living Symbiodinium may also limit the formation of novel associations (Finney et al. 2010; Cooper et al. 2011b), although a large diversity of Symbiodinium can be found in the water column and sediments (Hirose et al. 2008; Manning and Gates 2008; Pochon and Gates 2010; Pochon et al. 2010; Takabayashi et al. 2012; Nitschke et al. 2014) and on benthic macroalgae near coral reefs (Porto et al. 2008; Venera-Ponton et al. 2010).

\subsubsection{Partner recognition}

In order to establish the symbiosis, both host and symbiont first have to recognise each other. Well-studied symbioses, such as that between the bobtail squid, Euprymna scolopes, and its bioluminescent bacterium, Vibrio fischeri, provide evidence that microbe infection mechanisms play a role in symbiont discrimination (Nyholm and McFall-Ngai 2004; Weis 2008; Detournay et al. 2012). This involves molecular signalling and cell surface interactions, and inter-microbe ecological interactions; a series of complex steps known as 'winnowing' (Nyholm and McFall-Ngai 2004). Studies during the onset of cnidarian-dinoflagellate endosymbioses suggest that a similar mechanism to winnowing occurs, as occurs between: the green hydroid Hydra viridis and the chlorophyte alga Chlorella (Muscatine et al. 1975; Jolley and Smith 1978); the scyphozoan Cassiopeia xamachana and its dinoflagellate symbiont Symbiodinium microadriaticum (Colley and Trench 1983; Fitt and Trench 1983); and various 
other anthozoans and Symbiodinium spp. (Fitt and Trench 1981; Davy et al. 1997a; Coffroth et al. 2001; Rodriguez-Lanetty et al. 2006b; Poole et al. 2016). Biochemical and genomic studies have identified and characterised glycoproteins on the cell surface of dinoflagellate symbionts in culture, in the scleractinian coral Acropora tenuis (Bay et al. 2011) and soft coral Sinularia lochmodes (Jimbo et al. 2000; Jimbo et al. 2013), as well as lectins on the surface of host cells in Aiptasia sp. (Lin et al. 2000) and Fungia scutaria larvae (Wood - Charlson et al. 2006) and other symbiotic cnidarians (Jimbo et al. 2000; Wood - Charlson et al. 2006; Wood-Charlson and Weis 2009; Jimbo et al. 2010; Jimbo et al. 2013). Irrespective of horizontal or vertical transmission, studies indicate that cell surface signalling may play a significant role in the success of symbiosis, perhaps acting not only during the recognition stage but also during long-term symbiosis maintenance (Jolley and Smith 1980; McAuley and Smith 1982b; Coffroth et al. 2001; Weis et al. 2001; Belda-Baillie et al. 2002; Baker 2003; Marlow and Martindale 2007; Dunn and Weis 2009).

\subsubsection{Symbiont sorting}

Following host-symbiont surface recognition, free-living Symbiodinium cells are thought to be phagocytosed via the cells of the gastrovascular cavity (Figure 1.3) (McNeil and Smith 1982). As part of this process, the algal cells are then internalised by a host-derived vesicle (Smith and Douglas 1987; Wakefield and Kempf 2001; Fransolet et al. 2012). A sorting phase ensues, involving a wide range of cellular processes necessary for the maturation of Symbiodiniumcontaining phagosomes into functional symbiosomes, which serve to optimise the mutual exchange of compounds (Fitt and Trench 1983; Chen et al. 2003), although the exact cellular mechanisms involved in intracellular establishment and persistence are yet to be completely resolved (Peng et al. 2010; Davy et al. 2012). Competent Symbiodinium types may establish themselves by essentially avoiding host recognition, either circumventing or modulating the host immune responses that are normally triggered when non-competent types are encountered (Voolstra et al. 2009; Schnitzler and Weis 2010; Mohamed et al. 2016). Current research suggests that the algal cells have a mechanism to avoid host-lysosome digestion by preventing the fusion of symbiont-containing phagosomes with host-lysosomal cells; this is supported by the presence of Rab5 and absence of Rab7 expression during endosymbiosis establishment in Aiptasia sp. (Chen et al. 2003; Chen et al. 2004; Fransolet et al. 2012). Rab5 is involved in early endocytic compartmentalisation and potentially participates in persistence of Symbiodinium in the host, while Rab7 usually participates in late acidic endocytic and phagocytic pathways (Vitelli et al. 1997; Chen et al. 2003; Chen et al. 2004; Fransolet et al. 
2012). Furthermore, comparisons between symbiotic and aposymbiotic sea anemones (Anemonia viridis) show an upregulation in the gene sym32, thought to be a member of the fasciclin 1 family of cell adhesion and signalling proteins, which may therefore also play a role in recognition and tolerance of symbionts (Ganot et al. 2011).

If the symbiosis is compatible, the maintenance of a stable symbiosis requires the regulation of symbiont biomass so that host and symbiont cells live in dynamic equilibrium, including processes involved in the inhibition of cell growth and division, and the degradation and expulsion of excess symbionts (Muscatine and Pool 1979; Baghdasarian and Muscatine 2000; Fujise et al. 2013). This helps to maintain an optimal density of symbionts in host cells; one that is high enough to provide sufficient metabolic contribution while not over-growing host cells.

Meanwhile, incompatible symbionts are removed; this likely involves mechanisms such as host and symbiont cell apoptosis, a programmed cell death mechanism that is thought to be initiated by caspase signalling in cnidarians (Dunn et al. 2002; Dunn and Weis 2009; Tchernov et al. 2011; Dani et al. 2016). Autophagy is a cellular process for the degradation of cystolic components inside lysosomes, and is another programmed cell death mechanism. Autophagy of Symbiodinium is thought to occur via the conversion of symbiosomes to phagolysosomes resulting in the degradation of the symbionts (recently termed "symbiophagy") (Downs et al. 2009). Other mechanisms likely include gastrodermal cell detachment (Gates et al. 1992; Sawyer and Muscatine 2001), Symbiodinium exocytosis (Steen and Muscatine 1987; Downs et al. 2013), in situ symbiont cell degradation (Brown et al. 1995), or necrosis of both Symbiodinium (Strychar et al. 2004; Strychar and Sammarco 2009) and host cells (Dunn et al. 2002; Dunn et al. 2007).

Little else is known of the cellular processes involved in symbiosis recognition and maintenance, but one important consideration is the role played by photosynthetic products (Weis et al. 2008; Yellowlees et al. 2008; Weis and Allemand 2009; Davy et al. 2012). For example, studies of the symbiotic green Hydra show that two different strains of Chlorella that release vast amounts of maltose were more successful at colonisation than were those strains that release little or no maltose, or when photosynthesis was inhibited (Hohman et al. 1982; McAuley and Smith 1982a; Mews and Smith 1982). Similarly, trehalose was suggested to be a chemical attractant exuded by Symbiodinium to promote the establishment of symbiosis with larvae of the coral Fungia scutaria, and as an initiator of feeding behaviour (Hagedorn et al. 2015). 


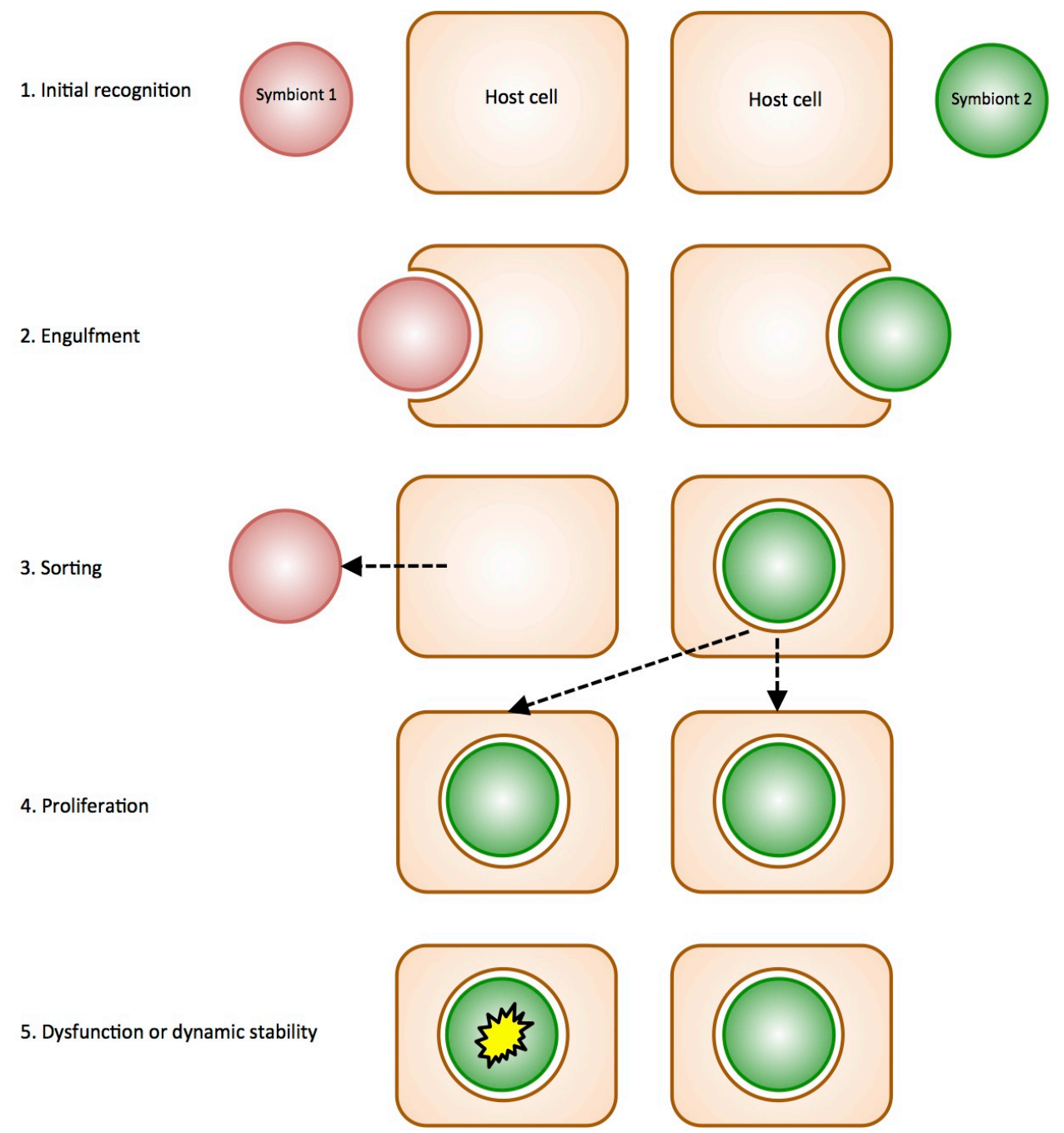

Figure 1.3 The phases of symbiosis uptake and establishment in the cnidarianSymbiodinium symbiosis. (1) Initial recognition of the algal symbiont cell; (2) Symbiont engulfment by the host cell; (3) Dynamic sorting of the symbionts leading either to rejection of the symbiont (red symbiont) or acceptance (green symbiont); (4) Proliferation of the symbiont via cell division within the host tissues; (5) Symbiosis breakdown (left) or dynamic stability, where the symbiont population is maintained at a steady density (right). For simplicity, not all the possible cellular events are represented here; for more detailed descriptions of these events, see in the main text. (Figure adapted from Davy et al., 2012.)

\subsection{Specificity}

Specificity within a symbiosis refers to the taxonomic range of partners with which an organism associates. Many Symbiodinium types can be categorised as generalists, associating with a wide variety of host species, while others are considered specialists, associating with one or a very few host species. For example, types B1, C1, and C3 have been found in a variety of coral hosts, while types B7 and C57a have only been found to associate with Madracis spp. and Millepora spp., respectively (LaJeunesse 2002; LaJeunesse 2005; LaJeunesse et al. 2010b). Despite the apparent generalist, flexible nature of many types, host-specificity within ITSdelineated types is becoming more apparent. For example, type B1 is found to associated with a number of Caribbean octocorals (LaJeunesse 2002), but phylogenetic comparison of 
microsatellite sequences from the type B1 populations revealed fine-scale specificity between Caribbean octocorals and these algae (Santos et al. 2004).

Many corals typically form stable mutualisms with one or a very few dominant Symbiodinium types (Coffroth and Santos 2005), but can also host a number of compatible low abundant symbionts comprising a background population (Fay and Weber 2012), the detection of which has improved with recent molecular technological advancements, such as next-generation amplicon sequencing, and qPCR (e.g. Boulotte et al. 2016). Meanwhile, some corals can form symbioses with multiple dominant Symbiodinium types, which may vary depending on life stage, depth, thermal history of the reef and locality (reviewed in Baker 2003). Nevertheless, host-symbiont combinations can be very stable, even if additional symbiont types are available (Hartle-Mougiou et al. 2012). For example, Fungia scutaria colonies originally transplanted to the Caribbean in the 1960 s continued to host symbiont type $\mathrm{C} 1 \mathrm{~b}$ found in conspecifics in the native range after 35 years, indicating remarkable longevity and specificity (LaJeunesse et al. 2005). However, an improvement in the detection of low abundant background populations suggests specificity is rarely absolute, and that in the coral-algal symbiosis, 'flexibility' and 'specificity' are perhaps relative terms (Silverstein et al. 2012; Boulotte et al. 2016). Furthermore, this paradigm depends on the genetic resolution used to resolve distinct symbiont types (Wham and LaJeunesse 2016; Howells et al. 2016; Smith et al. 2017; Thornhill 2017).

Nevertheless, the underlying mechanisms of symbiosis specificity and flexibility are unclear, although they are likely to involve the 'winnowing' mechanism described above. This would likely involve host immune responses and modulation of the response by the symbiont. Indeed, symbionts may avoid immune response as suggested by a lack in dramatic changes in host gene expression when colonised with competent symbionts (Voolstra et al. 2009; Schnitzler and Weis 2010; Mohamed et al. 2016). In contrast, exposure to non-competent symbionts stimulated the upregulation of numerous immune response genes (Voolstra et al. 2009). Similarly, aposymbiotic Fungia scutaria larvae colonised with a heterologous symbiont type elicited an immune response (as evident from caspase activity) that resulted in the rapid removal of these symbionts (Dunn and Weis 2009). Furthermore, patterns of specificity or flexibility in a particular symbiosis have appeared at the local, but not regional scale, possibly because of limitations in Symbiodinium dispersal and zonation (Finney et al. 2010; Cooper et al. 2011b).

Experimental manipulation of the symbiosis between cnidarians and Symbiodinium is crucial to advancing the understanding of the cellular mechanisms involved in host-symbiont interactions. The anemone Aiptasia sp. is a model for cnidarian-dinoflagellate symbiosis, and 
notably it can be rendered aposymbiotic (i.e. dinoflagellate-free) and re-infected with a range of Symbiodinium types (Belda-Baillie et al. 2002; Schoenberg and Trench, 1980; Starzak et al., 2014). Various methods exist for generating aposymbiotic hosts, including temperature stress followed by dark treatment and/or chemical photosynthesis inhibition; however, they can be hugely time consuming and not wholly effective (Belda-Baillie et al. 2002; Schoenberg and Trench, 1980; Starzak et al. 2014; Wang and Douglas, 1998; Xiang et al. 2013). Recently, Wang et al. (2012) described a method of menthol-induced bleaching in the corals Isopora palifera and Stylophora pistillata, and the development of a method using menthol for production of aposymbiotic Aiptasia will assist greatly in our understanding of the symbiosis.

Meanwhile, nutrient exchange may also play a role in determining partner combinations. In particular the quality and quantity of released photosynthetic products may act as recognition signals, as in the Hydra symbiosis (Hohman et al. 1982; McAuley and Smith 1982a). Simultaneously, further downstream of initial colonisation, metabolite exchange may influence the nutritional potential of the symbiosis (Stat et al. 2008; Cantin et al. 2009; Baker et al. 2013; Starzak et al. 2014; Pernice et al. 2015). These observations all imply that metabolic exchange has an impact on the stability of some cnidarian-Symbiodinium associations and therefore deserves further attention.

\subsection{Metabolic exchange}

Given the oligotrophic nature of the waters surrounding coral reefs, it comes as no surprise that nutritional exchange is the fundamental basis for this symbiosis; the dinoflagellates provide photosynthetic products which play a significant role in host nourishment and physiology, while in return the host provides its endosymbionts with sufficient nutrient pools for photosynthesis and respiration (Muscatine and Porter 1977). The association therefore involves tight nutrient recycling between the partners, with the bilateral exchange of metabolites as well as the synthesis of novel metabolites not produced by either organism independently (Figure 1.4) (Davy et al. 2012).

\subsubsection{Host-supplied inorganic nutrients}

In order for photosynthesis to occur, the algal cells must have access to dissolved inorganic carbon (DIC; usually in the form of carbon dioxide, $\mathrm{CO}_{2}$ ), as well as a suitable source of dissolved inorganic nitrogen (DIN), phosphorus, and other organic nutrients (Yellowlees and Warner 2003). Ultimately, $\mathrm{CO}_{2}$ is photosynthetically fixed and a range of organic compounds, including amino acids, are synthesised by the dinoflagellate. 


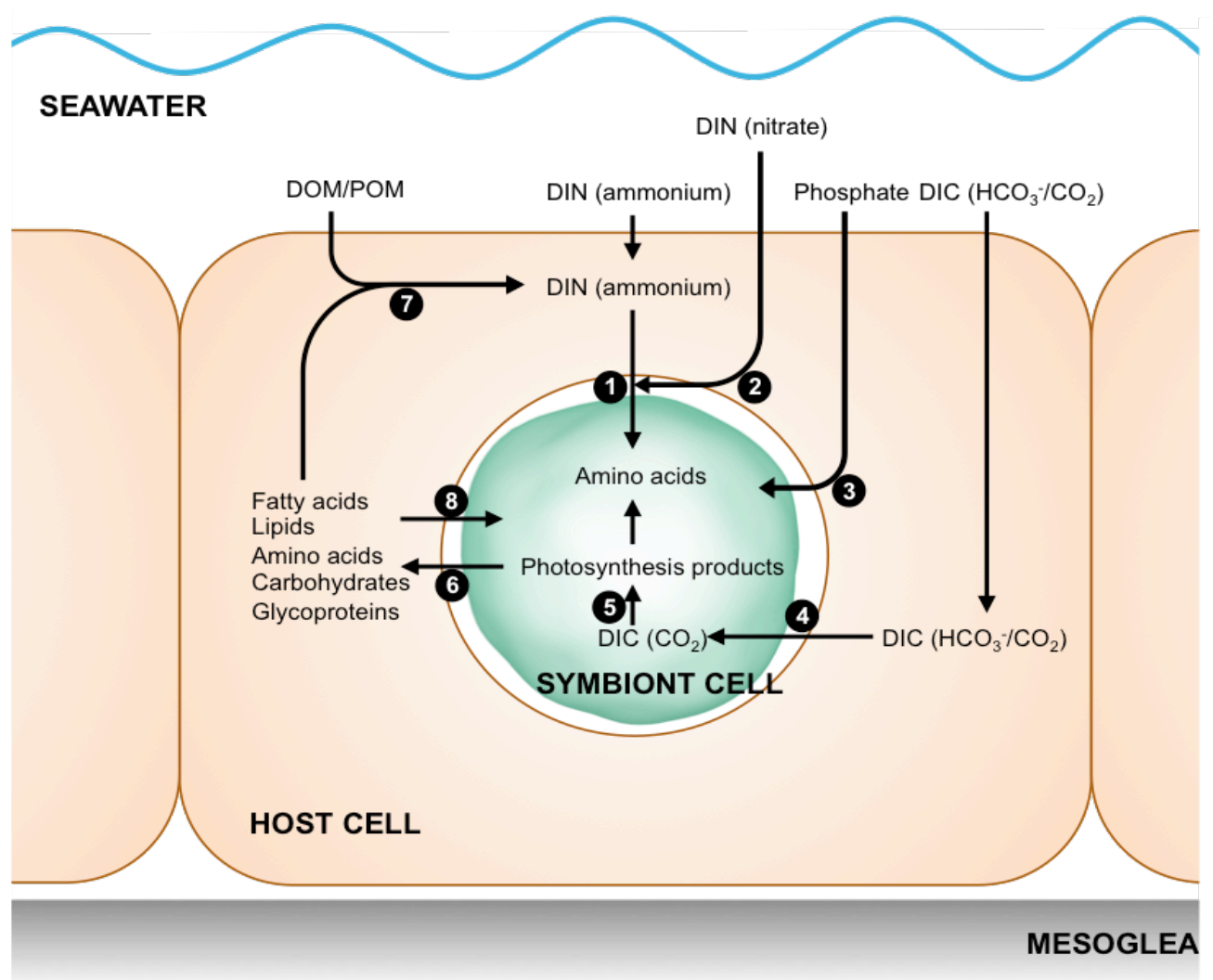

Figure 1.4 Summary schematic of nutritional acquisition and exchange in the cnidariandinoflagellate symbiosis. (1) Nitrogen is assimilated by both the host and algal cell, either from the surrounding seawater or from excretion. (2) Nitrate is acquired from the seawater, but only the symbiont can convert it to ammonium. (3) Similarly, phosphate is taken up from the seawater, and can be assimilated by the symbiont. (4) Dissolved inorganic carbon (DIC) is acquired as either $\mathrm{CO}_{2}$ from the seawater or host metabolism/calcification, or as bicarbonate from the seawater, which is then converted to $\mathrm{CO}_{2}$ in the symbiont cell. (5) $\mathrm{CO}_{2}$ is then photosynthetically fixed. (6) Some of the synthesised compounds (including amino acids) are used for symbiont metabolism, while a proportion is translocated to the coral host. (7) These compounds either support host metabolism alongside dissolved organic material (DOM) and particulate organic material (POM), or (8) are translocated back to the symbionts in the original form or as products of metabolism. (Figure adapted from Davy et al. 2012.)

For free-living algal cells and other marine autotrophs, carbon is normally obtained from the ambient seawater via carbon-concentrating mechanisms (CCM), which often include carbonic anhydrases (CAs) to catalyse the dehydration of bicarbonate $\left(\mathrm{HCO}_{3}{ }^{-}\right)$into $\mathrm{CO}_{2}$ (Whitney and Yellowlees 1995). Indeed, Symbiodinium have been show to use CAs as part of a CCM during symbiosis (Bertucci et al. 2013). However, in a symbiotic system, the host must also have CCMs to convert $\mathrm{HCO}_{3}^{-}$into $\mathrm{CO}_{2}$, and hence facilitate the delivery $\mathrm{CO}_{2}$ to the symbiont (Leggat et al. 2002; Barott et al. 2015). These mechanisms provide a partial pressure of $\mathrm{CO}_{2}$ in the immediate surroundings of the symbiont cells that is high enough to support photosynthetic carbon fixation (Venn et al. 2008; Allemand et al. 2011). The role of the host coral in an algal CCM is yet unclear, although it has been shown that host cells acidify the symbiotic microenvironment in order to promote photosynthesis (Barott et al. 2015), and coral CAs may 
also be involved (Isa and Yamazato 1984; Weis et al. 1989; Krupp and Weis 2006; Bertucci et al. 2011; Bertucci et al. 2013; Barott et al. 2015). Indeed, the increased activity and presence of CA in symbiotic versus aposymbiotic states (Weis et al. 1989; Weis 1991; Weis and Reynolds 1999), evidence of lower CA activity in shade-adapted and deeper-water holobionts (Weis et al. 1989), and more recent genomic evidence that CA activity is mediated by symbiotic state (Ganot et al. 2011), all advocate a role for CA activity in the cnidarian-dinoflagellate symbiosis. Nevertheless, its exact cellular locations remain unclear, though it is likely associated closely with the symbiosome membrane complex (Al-Moghrabi et al. 1996; Bertucci et al. 2011).

In contrast to DIC, under typical conditions in the oligotrophic waters which surround coral reefs, levels of DIN and phosphorus are low $(<1 \mu \mathrm{M})$, however in order to fuel effective symbiont cell function and growth, both elements must be available to the dinoflagellate (Figure 1.4) (Miller and Yellowlees 1989; Smith and Muscatine 1999). DIN in seawater is predominantly available as ammonium $\left(\mathrm{NH}_{4}^{+}\right)$and nitrate $\left(\mathrm{NO}_{3}^{-}\right)$. Ammonium is usually considered to be the preferential source of DIN for symbiotic reef corals, followed by nitrate (D'Elia et al. 1983; Grover et al. 2008). $\mathrm{NH}_{4}^{+}$may be directly assimilated via the glutamine synthetase/glutamine:2-oxoglutare aminotransferase (GS/GOGAT) enzymatic cycle (Swanson and Hoegh-Guldberg 1998; Roberts et al. 1999; Roberts et al. 2001; Pernice et al. 2012). Ammonium can also be directly assimilated by the cnidarian host via the action of GS and/or glutamate dehydrogenase (GDH) enzymes (Miller and Yellowlees 1989; Yellowlees et al. 1994; Wang and Douglas 1998). Additionally, the catabolism of nitrogenous compounds ultimately leads to the generation of $\mathrm{NH}_{4}{ }^{+}$waste that can then be assimilated by the dinoflagellate back into amino acids, completing the process of "nitrogen recycling" in the symbiosis (Wang and Douglas 1999; Davy et al. 2012; Pernice et al. 2012). In contrast, nitrate ions can only be assimilated by the symbiont because the coral host tissue lacks the nitrate reductase enzymes in a process that requires reduced ferredoxins (Fd) from the photosynthetic transport chain (Crossland and Barnes 1977; Tanaka et al. 2006; Kopp et al. 2014).

Dissolved inorganic phosphorus (DIP) (mainly phosphate) is the most accessible form of phosphorus to the symbiosis (Björkman and Karl 1994), but they can also take up dissolved organic (DOP) and particulate organic phosphorus (POP) (Ferrier-Pagès et al. 2016). DOP may be taken up by host heterotrophy, but it must be hydrolysed into DIP by phosphatases before it can be absorbed (Ferrier-Pagès et al. 2016), whereas DIP is likely concentrated and transported from surrounding seawater by carrier-mediated active transport in the coral host (Jackson and Yellowlees 1990; Godinot et al. 2009; Ferrier-Pagès et al. 2016). 
As both nitrogen and phosphorus are essential for symbiont growth and division, and given that Symbiodinium cells in hospite typically have slower rates of growth and division relative to when they are under optimal conditions in vitro (Miller and Yellowlees 1989; Muscatine et al. 1989a; Smith and Muscatine 1999; Davy et al. 2012), the host may manipulate the supply of these elements to control symbiont proliferation and maintain optimal rates of mobile product translocation (see Section 1.5.4) (Cook et al. 1988; Falkowski et al. 1993; Smith and Muscatine 1999; Kopp et al. 2013; Jiang et al. 2014).

\subsubsection{Symbiont-supplied photosynthate}

The photosynthetically-fixed carbon synthesised by the symbiont will have a number of fates: it can be metabolised or stored by the algae, or translocated to the host. The first evidence for the translocation of metabolites in the form of photosynthetically-fixed carbon from symbiont to host was derived from incubating the sea anemone Anthopleura elegantissima in ${ }^{14} \mathrm{C}$ bicarbonate and subsequent visualisation of radiolabel incorporation within anemone host tissues via autoradiography (Muscatine and Hand 1958). This pioneering experiment gave direct evidence for the incorporation of carbon products into the tissues of the host, and led to decades of research in this field of cnidarian-dinoflagellate symbiosis. Under functional conditions, over $40 \%$ (and potentially $>99 \%$ ) of the symbiont-derived photosynthate is translocated to the host, exceeding the host's normal metabolic requirements (Muscatine and Cernichiari 1969; Tremblay et al. 2014). The carbon-rich translocated products contribute to host respiratory pathways and provide energy-rich stores, while both carbon-rich and nitrogenrich compounds are utilised in biosynthesis pathways such as calcification, growth and reproduction (Tanaka et al. 2006; Kopp et al. 2015). Nevertheless, there is still great uncertainty about the quality and quantity of this translocated photosynthate (Table 1.1) (Venn et al. 2008; Gordon and Leggat 2010; Burriesci et al. 2012; Davy et al. 2012; Kopp et al. 2015; Hillyer et al. 2016a).

${ }^{14} \mathrm{C}$-bicarbonate radiotracer methodology was first used to label intracellular and released photosynthetic products (in the form of maltose) by Chlorella to the Hydra viridis host (Muscatine 1965). The photosynthate of isolated Symbiodinium was analysed using this same radiotracer combined with chromatographic techniques, and identified translocated-carbon products to be generally in the form of glycerol, with smaller pools of glucose, fumarate, succinate, malate, citrate, and glycolate (Muscatine 1965,1967; Von Holt and Von Holt 1968b; Muscatine and Cernichiari 1969; Lewis and Smith 1971; Trench 1971a,b,c). However, the identity of photosynthetic products was investigated with respect to the extracellular and 
intracellular composition of freshly isolated symbionts, meaning that the identifications may not be representative of the intact symbiosis (Sutton and Hoegh-Guldberg 1990; Allwood et al. 2012). On the other hand, studying mobile compounds translocated within an intact cnidarian holobiont is confounded by the fact that the algal cells are intracellular, and on-going metabolism and photosynthesis are likely to impede the identification and quantification of metabolic exchange. Lewis and Smith (1971) first attempted to identify the translocated compounds by supplying potential translocated compounds to a range of cnidarians in a ${ }^{14} \mathrm{C}$ labelled intact symbiosis. As the supplied compounds subsequently saturate all sites of uptake in the host, the corresponding ${ }^{14} \mathrm{C}$-labelled compounds are released by the symbionts to the surrounding medium. They also concluded glucose, glycerol, and alanine were the dominant forms of translocated carbon (Lewis and Smith 1971). More recently, Whitehead and Douglas (2003) attempted to identify the compounds exchanged within the intact symbiosis of Anemonia viridis by labelling exogenously supplied metabolites under non-photosynthesising conditions and comparing the incorporation of these metabolites into host tissues when under photosynthesising conditions. When a labelled metabolite appeared in both treatments, the exogenous compounds were considered indicative of those translocated. This study detected labelling of glucose, as well as the organic acids succinate and fumarate, but crucially there was no evidence of glycerol translocation (Whitehead and Douglas 2003). Similarly, when Burriesci et al (2012) labelled the sea anemone Aiptasia pallida with a ${ }^{13} \mathrm{C}$ stable isotope tracer, they detected rapid labelling of glucose in host pools but not glycerol. Conversely, a non-targeted ${ }^{13} \mathrm{C}$ tracer-fate study by Hillyer et al (2016) detected both labelled glucose and glycerol in the host pools of Aiptasia sp. and the scleractinian coral Acropora millepora, but comparatively less glycerol was detected. Glucose, rather than glycerol, is therefore now considered as the major translocated metabolite in the intact symbiosis.

The identification of additional translocated compounds comes from an improvement in methodologies, such as studies investigating the differences in metabolic composition between hosts in an aposymbiotic versus symbiotic state (Papina et al. 2003; Whitehead and Douglas 2003; Burriesci et al. 2012; Lehnert et al. 2014), and variations in response to different irradiance regimes (Wang and Douglas 1999; Chen et al. 2012). More recent approaches have analysed the host genome (Shinzato et al. 2011), compared of the gene expression profiles of anemones in aposymbiotic versus symbiotic states (Lehnert et al. 2014), and profiled the metabolome of the host tissues when in symbiosis (Whitehead and Douglas 2003; Burriesci et al. 2012), and in response to different thermal regimes (Hillyer et al. 2016a; Hillyer et al. 2016b) and ocean acidification stressors (Sogin et al. 2016). Furthermore, transcriptomic and 
proteomic analyses have attempted to identify proteins involved in the transport of metabolites as a means to understand nutritional exchange in the symbiosis (Peng et al. 2010; Lehnert et al. 2014; Oakley et al. 2016).

Table 1.1 Example table of our current understanding for translocated compounds in the cnidarian-dinoflagellate symbiosis.

\begin{tabular}{|c|c|c|c|c|}
\hline \multirow[t]{2}{*}{ Metabolite } & \multicolumn{2}{|c|}{ Translocation direction } & \multirow[t]{2}{*}{ Host organism } & \multirow[t]{2}{*}{ References } \\
\hline & $\begin{array}{l}\text { Host to } \\
\text { symbiont }\end{array}$ & $\begin{array}{l}\text { Symbiont } \\
\text { to host }\end{array}$ & & \\
\hline \multicolumn{5}{|l|}{ Sugar } \\
\hline Maltose & & 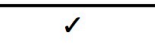 & Hydra & Muscatine 1965 \\
\hline $\begin{array}{l}\text { Glucose and other } \\
\text { hexoses }\end{array}$ & & $\checkmark$ & $\begin{array}{l}\text { Coral, anemones, } \\
\text { tridacnid clams, } \\
\text { jelly-fish and } \\
\text { hydrozoans }\end{array}$ & $\begin{array}{l}\text { Muscatine et al. 1967; Bil et al. 1991; } \\
\text { Ishikura et al. 1999; Whitehead and } \\
\text { Douglas 2003; Burriesci et al. 2012; } \\
\text { Lehnert et al. } 2014\end{array}$ \\
\hline \multicolumn{5}{|l|}{ Sugar alcohols } \\
\hline Glycerol & & 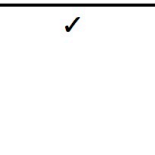 & $\begin{array}{l}\text { Coral, anemones, } \\
\text { tridacnid clams and } \\
\text { hydrozoans }\end{array}$ & $\begin{array}{l}\text { Muscatine 1967; Muscatine and } \\
\text { Cernichiari 1969; Lewis and Smith 1971; } \\
\text { Trench 1971; Davies 1984; Sutton and } \\
\text { Hoegh-Guldberg } 1990\end{array}$ \\
\hline \multicolumn{5}{|l|}{ Sterols } \\
\hline Cholesterol & & 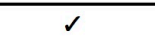 & Anemones & Ganot et al. 2011 \\
\hline \multicolumn{5}{|l|}{ Amino acids } \\
\hline Alanine & & 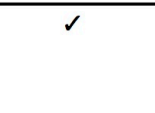 & $\begin{array}{l}\text { Coral, anemones, } \\
\text { jelly-fish and } \\
\text { hydrozoans }\end{array}$ & $\begin{array}{l}\text { Muscatine 1967; Lewis and Smith 1971; } \\
\text { Trench 1971; Hillyer } 2016\end{array}$ \\
\hline Glycine & $\checkmark$ & $\checkmark$ & Zoanthids & Von Holt 1968; Hillyer 2016 \\
\hline $\begin{array}{l}\text { Mycosporine-like amino } \\
\text { acids e.g. cysteine }\end{array}$ & & $\checkmark$ & Coral & Shinzato et al. 2011; Hillyer 2016 \\
\hline Other amino acids & $s$ & $\checkmark$ & Anemones & Wang and Douglas 1999; Hillyer 2016 \\
\hline \multicolumn{5}{|l|}{ Fatty acids } \\
\hline Fatty acids & 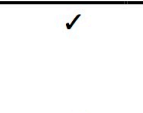 & $\sqrt{2}$ & Coral & $\begin{array}{l}\text { Patton et al. 1977; Yamashiro et al. 1999; } \\
\text { Papina et al. 2003; Imbs et al. 2010; Imbs } \\
\text { et al. 2014; Hillyer } 2016\end{array}$ \\
\hline Lipids & $\checkmark$ & $\checkmark$ & Coral, anemones & $\begin{array}{l}\text { Kellogg and Patton 1983; Yamashiro et al. } \\
\text { 1999; Oku et al. 2003; Imbs et al. 2010; } \\
\text { Imbs et al. 2014; Kopp et al. } 2015\end{array}$ \\
\hline \multicolumn{5}{|l|}{ Other organic acids } \\
\hline Glycolic acid & & $\sqrt{2}$ & $\begin{array}{l}\text { Coral, tridacnid } \\
\text { clams }\end{array}$ & Muscatine et al. 1967 \\
\hline Acetate & $s$ & $\checkmark$ & Coral, zoanthids & $\begin{array}{l}\text { Von Holt and Von Holt 1968; Patton et al. } \\
1977\end{array}$ \\
\hline $\begin{array}{l}\text { Lactate, citrate, } \\
\text { ketoglutarate, malate, } \\
\text { pyruvate }\end{array}$ & & $\checkmark$ & Coral, zoanthids & $\begin{array}{l}\text { Von Holt and Von Holt 1968; Lewis and } \\
\text { Smith } 1971\end{array}$ \\
\hline Succinate & & $s$ & $\begin{array}{l}\text { Coral, anemones, } \\
\text { zoanthids }\end{array}$ & $\begin{array}{l}\text { Von Holt and Von Holt 1968; Lewis and } \\
\text { Smith 1971; Whitehead and Douglas } \\
\text { 2003; Lehnert et al. } 2014\end{array}$ \\
\hline Fumarate & & $\checkmark$ & Anemones & Whitehead and Douglas 2003 \\
\hline
\end{tabular}




\subsubsection{1. $\quad$ Sugars and sterols}

Glucose can enter eukaryotic cells through either of two transporter proteins: the facilitated glucose transporter (GLUT) or the sodium-coupled glucose transporter (SGLT). Proteins sequences from both the GLUT and SGLT families have been identified in the genome of the cnidarian Aiptasia pallida (Baumgarten et al. 2015). While transporters specific to the symbiosome are yet to be confirmed, gene expression analysis detected multiple transporters that were upregulated in a symbiotic versus aposymbiotic state, including the GLUT8 protein (Lehnert et al. 2014). Niemann-Pick C type 2 proteins are associated with intracellular sterol transporters and were previously identified as being upregulated in the symbiotic versus aposymbiotic Aiptasia sp. (Dani et al. 2014; Lehnert et al. 2014). NPC2 proteins were subsequently found in the Aiptasia genome to be closely associated with symbiosomes (Dani et al. 2014), and therefore they may operate in sterol and lipid transport from symbiont to host (Lehnert et al. 2014; Oakley et al. 2014). The characterisation and expression patterns of nutrient transporter families involved in metabolite exchange will help to elucidate candidate translocated compounds, as occurred during the symbiosome characterisation of legumes (Day and Udvardi 1993; Day et al. 2001; Peng et al. 2010). The increasing availability of host and symbiont genomes will facilitate this task (Peng et al. 2010; Lehnert et al. 2014; Li et al. 2014).

\subsubsection{Amino acids and proteins}

Carbon metabolism yields carbon skeletons that can be used for other metabolic reactions, including the production of amino acids, fatty acids and lipids. However, many amino acids cannot be synthesized de novo by organisms and so must be sourced elsewhere, e.g. from endosymbiotic algae. The translocation of amino acids, such as alanine, was first observed by Trench (1971a-c), though this was based on photosynthate released by symbionts incubated in host homogenate, and many amino acids were at extremely low concentrations or may have been undetectable (Sutton and Hoegh-Guldberg 1990). Recent analysis of the Aiptasia transcriptome revealed that, like other animals, Aiptasia is able to synthesise eight of the 20 amino acids; the other 12 amino acids (or their precursors) they must receive from their diet, their dinoflagellate endosymbionts, or both (Wang and Douglas 1999; Lehnert et al. 2014). Several essential amino acids (e.g. histidine, isoleucine, leucine, lysine, phenylalanine, tyrosine and valine) have been observed in symbiotic Aiptasia sp., suggesting that they may be synthesised by the symbiotic algae and translocated to the sea anemone's tissues (Wang and Douglas 1999; Hillyer et al. 2016a). Stable isotope ${ }^{13} \mathrm{C}$-tracer fate metabolite profiling identified a number of amino acids in the host pools of Aiptasia sp. (Burriesci et al. 2012; 
Hillyer 2016) and Acropora millepora (Hillyer 2016), corroborating the translocation of symbiont-derived amino acids to the cnidarian host.

\subsubsection{Fatty acids and lipids}

Omega-3 and omega- 6 fatty acids cannot be synthesized by animals, as they cannot make a double bond past the $\Delta^{9}$ position, and thus they must obtain these essential fatty acids from plants or some bacteria. Indeed, autotrophic and heterotrophic organisms have different abilities to produce specific fatty acid groups (Dunn et al. 2012; Leal et al. 2013). Lipids and fatty acids are an important energy source, act as signal molecules and play a structural role in organisms, while the lipid/fatty acid composition of corals is directly coupled with photosynthesis (Oku et al. 2003a; Chen et al. 2012). It is therefore no surprise that lipids and fatty acids constitute another part of the organic carbon exchange within the coraldinoflagellate symbiosis (Papina et al. 2003; Kopp et al. 2015; Hillyer 2016), and the exchange of fatty acids has been suggested to be a requirement for establishment of the endosymbiosis (Wang et al. 2013).

Reciprocal translocation of fatty acids is evident from the detection of symbiont-derived fatty acids in host tissues (e.g. C18:4n-3, C22:5n-3 and C22:6n-3) and vice versa (C18:2n-7 and C22:5n-6) (Papina et al. 2003; Imbs et al. 2014). Additionally, support for the translocation of fatty acids was shown by the upregulation of scavenger receptor genes related to a fatty acid transport protein, and a putative carnetine transporter gene in symbiotic anemones (Lehnert et al. 2014). Fatty acid translocation and lipid stores are depleted in bleached corals, demonstrating a link between fatty acid translocation and symbiont density (Imbs and Yakovleva 2012; Hillyer et al. 2016a). Although corals possess the ability to obtain external sources of fatty acids and lipids via planktonic feeding, the presence of Symbiodinium-specific fatty acids and fatty acid membrane transporters, and studies demonstrating a significant decline of stored lipids in the absence of symbionts as a result of bleaching events, are indicative of the provision of fixed carbon in the form of fatty acids from the symbionts to the host (Hillyer 2016; Hillyer et al. 2016b).

Fatty acids are key constituents of lipids, which serve as key biomolecules in coral tissues, functioning in energy storage, membrane structure, cellular homeostasis and cell signalling (Rodrigues et al. 2008; Díaz-Almeyda et al. 2010; Dunn et al. 2012). Tropical corals contain $\sim 10-30 \%$ of dry tissue weight as lipids (Harland et al. 1993; Yamashiro et al. 1999) and corals have been shown to depend on these stored lipids to survive bleaching events (Rodrigues and Grottoli 2007; Rodrigues et al. 2008; Grottoli and Rodrigues 2011; Imbs and Yakovleva 2012; 
Kneeland et al. 2013). Subcellular visualisation of stable isotope-labelled translocated photosynthetic products in the reef coral Pocillopora damicornis accumulated in the coral's lipid droplets (Kopp et al. 2015). Lipids in the host's tissues are primarily stored as wax esters and triacylglycerols (Patton et al. 1977; Yamashiro et al. 1999; Imbs et al. 2010; Kopp et al. 2015). Lipid biogenesis is dependent upon the symbiotic status of a coral and its symbionts, and the translocation of lipid compounds from symbiont to host is postulated to be in the form of fatty lipid bodies (LBs) (Crossland et al. 1980; Chen et al. 2012).

\subsubsection{Other metabolites}

Along with carbohydrates, amino acids, fatty acids and lipids, other translocated compounds within cnidarian-algal symbioses include organic acids such as glycolic acid (Muscatine et al. 1967), acetate (Von Holt and Von Holt 1968a,b; Patton et al. 1977), lactate, citrate, ketoglutarate, malate, pyruvate (Von Holt and Von Holt 1968a; Lewis and Smith 1971), succinate (Von Holt and Von Holt 1968a; Lewis and Smith 1971; Whitehead and Douglas 2003; Lehnert et al. 2014), aspartate, glutamate (Wang and Douglas 1999), and fumarate (Whitehead and Douglas 2003). Organic acids are important intermediates of the TCA cycle (e.g. succinate), as well as providing nutrition (e.g. glycolic acid), and acting as precursors for protein (e.g. pyruvate) and fatty acid synthesis (acetate). Various glycoproteins may also be constituents of the translocated material, as identified on the cell surface of cultured Symbiodinium, and their cellular locations suggest that they may play a role in hostSymbiodinium specificity (Logan et al. 2010).

\subsubsection{Quantity of translocated carbon-rich photosynthate}

Under well-lit conditions, autotrophy is thought to be sufficient to support a coral host's respiratory demands (Muscatine et al. 1981; Davies 1991; Davy et al. 1996; Muller - Parker and Davy 2001; Hughes and Grottoli 2013; Starzak et al. 2014). For tropical corals, organic carbon supply can be met by autotrophy alone (Muscatine et al. 1984), however for temperate symbiotic cnidarians, additional heterotrophic sources of carbon are required to meet the host's respiratory demands (Falkowski et al. 1984), such as via the uptake of particulate and dissolved organic material from the surrounding environment (Lewis and Price 1975), mucus feeding, and predation on micro-organisms and zooplankton (Muscatine et al. 1984; Tremblay et al. 2015). In order to quantify how much carbon is translocated, one must consider the amount of photosynthetic carbon fixation, which is influenced by light regime and the particular irradiance of each day (Falkowski et al. 1984; Davies 1991), and how much of that fixed carbon is exported from the symbiont cell (Muscatine and Hand 1958; Trench 1971b,c,a; 
Muscatine et al. 1981). By evaluating the proportion of radiolabelled carbon in host tissues, Trench (1971a-c) was able to provide estimates of translocated fixed carbon in the sea anemone Anthopleura elegantissima, although this method has serious limitations in terms of on-going host metabolism and re-fixing of labelled intermediates that can affect the radiolabel signal (Davy et al. 2012), as well as problems with the variability of the ${ }^{14} \mathrm{C}:{ }^{12} \mathrm{C}$ ratio (Battey and Patton 1984; Muscatine 1990). As a result of these limitations, Muscatine et al. (1981) introduced an alternative method for quantifying translocation, based on the assumption that all fixed carbon not used by the symbiont to support its respiratory and growth demands is released to the host. However, this method too has limitations in terms of requiring prior knowledge of net photosynthesis, and respiration of both partners individually, in the intact symbiosis (Muscatine et al. 1983; Hawkins et al. 2016b). Moreover, this method assumes that carbon is not stored by the dinoflagellate cell (Muller-Parker et al. 1996), and relies on indirect estimations of symbiont growth rate derived from measures of the mitotic index (Smith and Muscatine 1986; Harland and Davies 1995). The proportion of fixed carbon that a symbiont provides for the host's carbon budget will depend on the host species, environmental conditions, bleached status, feeding regime and symbiont genotype (Cooksey and Cooksey 1972; Davy and Cook 2001; Loram et al. 2007; Hughes et al. 2010; Tremblay et al. 2013; Starzak et al. 2014). However, the methodological limitations described above make quantifications extremely tentative.

\subsubsection{Regulation and fate of metabolite translocation}

A number of mechanisms have been suggested to control the high rate of transfer from the symbiont to host: 1) Putative "host release factors" (HRFs) are one concept that has received much interest, however there are questions surrounding their exact identity and mode of operation (Muscatine et al. 1972; Gates et al. 1995; Wang and Douglas 1997; Grant et al. 1998; Withers et al. 1998; Gates et al. 1999; Cook and Davy 2001; Grant et al. 2001; Grant et al. 2006b), and whether they are, in fact, involved or not (Grant et al. 2003). Free amino acids and other small ( $<1 \mathrm{kDa}$ ) host compounds might act as HRFs (Gates et al. 1995), although more recent research has identified other cell-signalling molecules that could control photosynthate release and photosynthetic performance (Grant et al. 2001; Grant et al. 2006b; Grant et al. 2006a); 2) As the host is thought to maintain the symbiont in a state of nitrogen and phosphorus limitation, high $\mathrm{C}: \mathrm{N}: \mathrm{P}$ ratios will result in arrested symbiont growth and cell division. Subsequently, nitrogen deprivation leads to the accumulation of energy rich stores (such as lipid droplets) in Symbiodinium, which may in turn be made available to the host (Wang and Douglas 1998; Smith and Muscatine 1999); 3) Furthermore, the host maintains an 
acidic $\mathrm{pH}$ in the symbiosome space via the activity of vacuolar $\mathrm{H}^{+}$-ATPase proton pump, which promotes $\mathrm{CO}_{2}$ accumulation in the symbiont cell and a corresponding increase in photosynthetic rate and translocation of mobile products (Barott et al. 2015), aided via membrane-bound nutrient transporter mechanisms e.g. ABC transporters (Dudler and Miller 1988; Davy et al. 2012).

In addition, there is a lack of information relating to the spatial distribution of translocated compounds within tissues and cells, which is compounded by the intracellular location of the algal endosymbionts. The advent of high spatial resolution molecular imaging techniques that allow for the in situ analysis of the symbiosis, including matrix-assisted laser desorption ionization mass spectrometry imaging (MALDI-MSI) for the visualisation of labelled compounds in tissues and nanoSIMS for the visualisation of labelled compounds in cells, will enable the identification of the exact subcellular fate of translocated metabolites and may help to clarify the nutritional interactions in this symbiosis (Davy et al. 2012; Pernice et al. 2012; Kopp et al. 2013; Kopp et al. 2014).

\subsubsection{Taxonomic and environmental variation in metabolite profiles}

The composition and diversity of released low molecular weight compounds derived from photosynthesis, including sugars and amino acids, has been shown to vary among different symbioses and studies (Trench 1979; Miralles et al. 1989). Indeed, different symbiotic associations do not necessarily translocate the same compounds and/or in the same proportions (Trench 1979; Miralles et al. 1989; Bil et al. 1991; Loram et al. 2007; Starzak et al. 2014).

Metabolite profiling has revealed taxonomic and environmental variation in the metabolite profiles of the genus Symbiodinium (Klueter et al. 2015). Moreover, although different symbiont genotypes may produce similar metabolites, their proportions shift under different environmental conditions (Trench 1971c,a,b). Symbiont type has been shown to impact the functionality and autotrophic potential of the symbiosis, and heterologous symbionts may behave opportunistically, proliferating rapidly but in a manner that is energetically costly to the host (Starzak et al. 2014; Leal et al. 2015). Recent utilisation of nanoscale secondary ion mass spectrometry (nanoSIMS) has shown varying levels of metabolic capabilities between Symbiodinium types C and D within the same coral host (Pernice et al. 2015). This study shows a significantly higher level of assimilation and translocation in type $\mathrm{C}$ symbionts in hospite, aligning with previously suggested differential metabolic efficiencies of Symbiodinium genotypes (Rowan 2004; Cantin et al. 2009; Coffroth et al. 2010). 
Furthermore, the symbiont type may not only influence the amount of carbon, but also the identity of the compounds. In this regard, the lipid and fatty acid composition, and the enzymes controlling fatty acid metabolism and biosynthesis pathways, of isolated and cultured Symbiodinium cells varies between genotypes (Zhukova and Aizdaicher 1995; Zhukova and Titlyanov 2003; Tchernov et al. 2004; Klueter et al. 2015; Parkinson et al. 2016). Concurrently, host lipid content and the identity and abundance of translocated fatty acids are influenced by symbiont type (Zhukova and Titlyanov 2003; Cooper et al. 2011a; Cooper et al. 2011b). A change in lipid and fatty acid composition has been shown to reflect changes in nutrition, ecology and ultimately the health of corals (Oku et al. 2003b; Saunders et al. 2006), so symbiont type may affect the long-term survival of the holobiont.

\subsection{The role of Symbiodinium in the response of corals to climate change}

\subsubsection{Coral bleaching}

Environmental perturbations, such as extended periods of elevated sea surface temperature, can lead to loss of Symbiodinium cells or just their pigmentation, which gives the corals a "bleached" or paled appearance (Figure 1.5) (Hoeksema 1991; Glynn 1996; Brown 1997). Bleaching can incur physiological costs for the host coral as a result of loss of photosynthetic performance, which may lead to lower energy reserves, slower growth, decreased immunity and reduced reproductive rate in successive years (Little et al. 2004; Loram et al. 2007; Rodrigues and Grottoli 2007). Depending on the duration and severity of the bleaching, a coral will either regain its algal symbionts (and/or algal pigmentation) and recover, or die (McClanahan 2004; Montano et al. 2010). Owing to the large scale at which heat-induced coral bleaching and mortality can occur, it is considered a major extinction threat for many reef coral species (Carpenter et al. 2008).

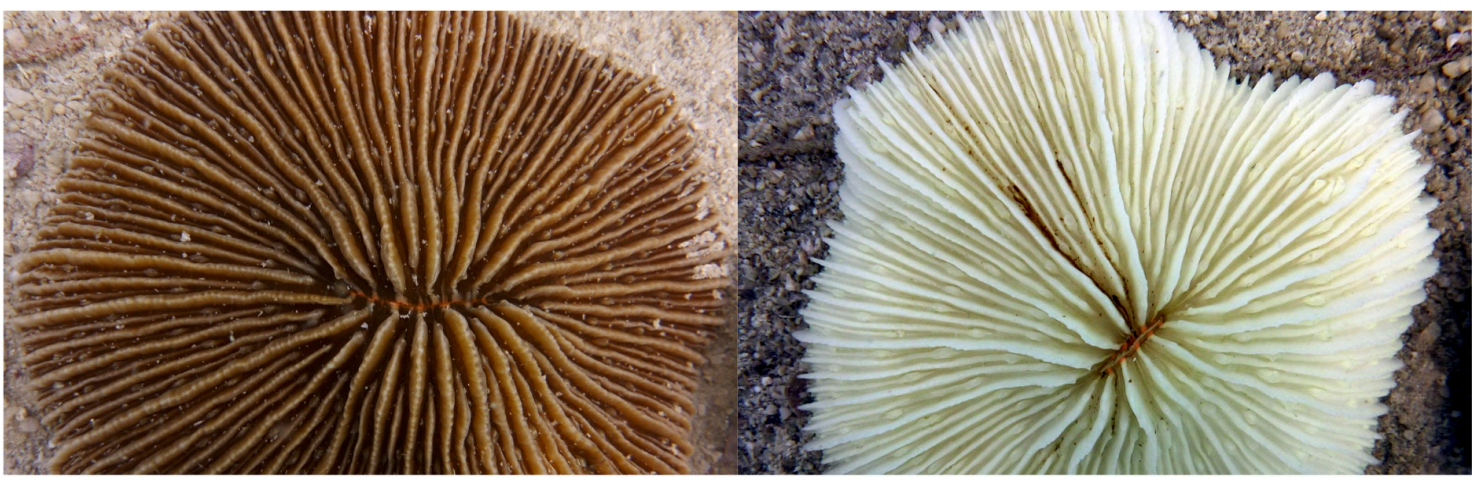

Figure 1.5 Coral bleaching. A healthy, non-bleached Fungia scruposa individual (left) and bleached individual (right). 


\subsubsection{Adaptive bleaching hypothesis}

A growing body of research suggests that a coral's response to environmental fluctuation is heavily influenced by the physiology of the resident symbiont population (Buddemeier and Fautin 1993; Rowan et al. 1997; Jones et al. 1998; Baker 2003; Fabricius et al. 2004; Rowan 2004; Tchernov et al. 2004; Berkelmans and Van Oppen 2006; Ulstrup et al. 2006; Jones et al. 2008; Sampayo et al. 2008b; van Oppen et al. 2009). For example, Porites lobata colonies harbouring type $\mathrm{C} 3$ are more susceptible to coral bleaching than those harbouring $\mathrm{C} 15$ (LaJeunesse et al. 2004; Barshis et al. 2010; Hume et al. 2013). Meanwhile, clade D symbionts have been show to be heat tolerant and provide thermal protection to the host (Rowan 2004; Berkelmans and Van Oppen 2006).

Therefore, provided mortality does not result, coral bleaching may present the opportunity for the endosymbiont community to change upon recovery (Berkelmans and Van Oppen 2006; Stat et al. 2009; Stat and Gates 2011; Boulotte et al. 2016). For instance, the relative abundance of each Symbiodinium type concurrently existing within the host can change in response to environmental conditions, causing a shift in the dominant symbiont known as symbiont "shuffling" (Figure 1.6) (Baker 2003; Cunning and Baker 2014; Kemp et al. 2014). Alternatively, it has been suggested that novel symbionts may be acquired from surrounding waters during bleaching, in a process known as symbiont "switching" (Baker 2003). These mechanisms were originally hypothesised in the 'adaptive bleaching hypothesis', and would require mechanisms for both novel symbiont acquisition and symbiont inheritance by the symbiosis, and some limited potential for this has been shown in Stylophora pistillata (Byler et al. 2013) and pocilloporid corals (Boulotte et al. 2016).

Gaining thermotolerance from changes in the symbiont population is likely to occur in a hostand Symbiodinium-specific manner. Some coral species show a long-lasting shift towards hosting thermally tolerant symbiont types that persist for the duration of the monitoring (Baker 2001; Berkelmans and Van Oppen 2006; Edmunds et al. 2014), while others establish a temporary change only to return to the original symbiont type later during recovery (Thornhill et al. 2006; Coffroth et al. 2010; LaJeunesse et al. 2010c). If corals are not able to switch, then acclimatisation and adaptation of the complement symbiont may provide thermotolerance e.g., thermotolerance diversity was identified within ITS type C1 (Howells et al. 2012).

However, symbiosis specificity is likely to restrict the ability for symbiont switching, shuffling, or both (Baker 2003; Goulet 2006; Oliver and Palumbi 2010). Indeed, some studies demonstrate that some corals do not change their symbionts in response to thermal stress 
(LaJeunesse et al. 2008; Sampayo et al. 2008b; Bellantuono et al. 2011). Furthermore, ecosystem-scale modelling raises into question whether host and symbiont traits might ameliorate environmental change, if the physiological costs outweigh the ecological benefits (Ortiz et al. 2013; Ortiz et al. 2014). For instance, corals harbouring symbionts from clade D demonstrate higher thermal tolerances than conspecifics hosting clade $\mathrm{C}$ members (Glynn et al. 2001; Berkelmans and Van Oppen 2006; Sampayo et al. 2008b; Stat and Gates 2011; Silverstein et al. 2015), however under non-stressful conditions, clade C symbionts show greater photosynthate contribution than clade D (Cantin et al. 2009), and higher growth rates were observed for clade $\mathrm{C}$ hosts (Cunning et al. 2015a). The host-symbiont combination may therefore influence the nutritional potential of the symbiosis, as well as its likelihood of survival and long-term success (Ackman et al. 1968; Volkman et al. 1989; Brown and Jeffrey 1992; Dunstan et al. 1992; McAuley 1994; Zhukova and Aizdaicher 1995; Zhukova and Titlyanov 2003; Bongaerts et al. 2010). Understanding the factors controlling different combinations of host and symbiont species, and the physiological and ecological implications of this possibility under different environmental regimes is therefore of paramount importance for understanding the potential for how corals, and the ecosystems they support, adapt to climate change (Mieog et al. 2009).

A

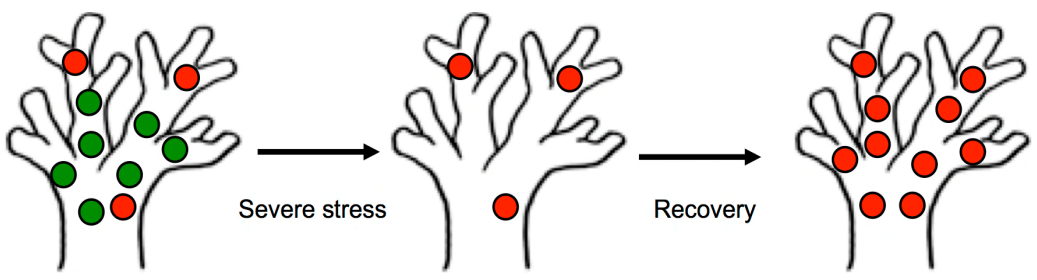

B

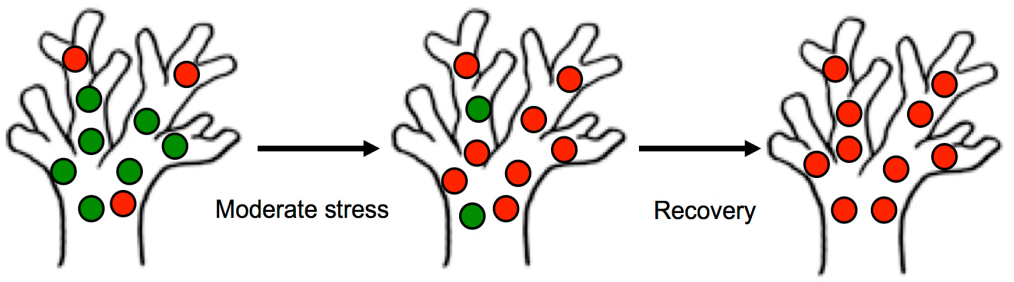

C

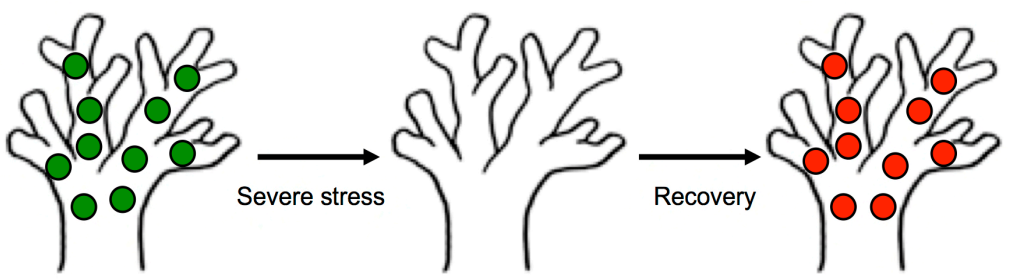

Figure 1.6 Schematic of coral bleaching and symbiont shuffling and switching. The coloured circles symbolise symbiont types. A change in dominant symbiont type from a heat sensitive (green) to a heat tolerant type (red) can occur via (A) the post-bleaching proliferation of a residual, thermally tolerant type (bleaching-induced symbiont shuffling); (B) gradual symbiont turnover in response to environmental change (dynamic symbiont shuffling); or (C) a switch to a new symbiont from an environmental source population following bleachingrelated disturbance. 


\subsection{Gaps in our knowledge}

The interest in the nutritional inter-relationship between cnidarians and their algal symbionts began over a century ago, when it was suggested that corals may obtain 'nutriment from the algae' (Duerden 1902). However, many of the key questions about the metabolism and metabolic integration of the cnidarian-algal symbiosis rose by researchers in the 1970s and early 1980s (Muscatine and Hand 1958; Trench 1971a,b,c; Muscatine et al. 1984) are still to be answered. Currently, little is known about the major translocated compounds, and even less is known about the more minor compounds translocated by Symbiodinium, perhaps complicated by the difficulties involved in analysing the intact symbiosis in a meaningful way and timeframe, with sufficient sensitivity. The major gaps in our understanding include:

1. The identity of the photosynthetic products translocated to the cnidarian host;

2. Whether the identity and quantity of translocation varies depending on the Symbiodinium genotype with which a host is associated;

3. The role of nutrient translocation in host-symbiont specificity.

\subsection{The application of metabolomics}

For a holistic understanding of the complex nutritional interactions within the cnidariandinoflagellate symbiosis and those which may lead to symbiont-specificity, it is essential to follow the response of the host to a conditional perturbation at the transcriptome (gene expression), proteome (protein composition) and metabolome (metabolite) levels (Gordon and Leggat 2010; Chaston and Douglas 2012; Meyer and Weis 2012; Lehnert et al. 2014). Comparative genomic, transcriptomic, proteomic and lipidomic methods have already started to shed light on the coral-algal symbiosis and its response to environmental perturbations (Fiehn 2001; Kuo et al. 2004; Barneah et al. 2006; Deboer et al. 2007; Voolstra et al. 2009; DeSalvo et al. 2010b; Kuo et al. 2010; Schnitzler and Weis 2010; Leggat et al. 2011a; Bayer et al. 2012; Schmeitzel et al. 2012; Schnitzler et al. 2012; Barshis et al. 2013; Shoguchi et al. 2013a; Voolstra 2013; Barshis et al. 2014; Lehnert et al. 2014). Meanwhile, metabolomics is the comprehensive analysis by which all the metabolites of an organism are identified and quantified. This analysis has contributed to our understanding of the complex molecular interactions in biological systems, including symbiosis studies (Bino et al. 2004; Schliemann et al. 2008; Rasmussen et al. 2009). Since metabolism is closest to the phenotype, study of the metabolome may be able to qualitatively and quantitatively describe the dynamics of the 
symbioses, such as the metabolic requirements that restrict the formation of novel associations, or the nutritional response to thermal stress (Guy et al. 2008).

\subsubsection{GC-MS analysis}

Metabolite profiling with the use of gas chromatography (GC) coupled with mass spectrometry (MS) is a powerful tool for accurately identifying and quantifying metabolites in a biological sample, and has been used, for example, to isolate unsaturated very long-chain fatty acids from the marine sponge Halichondria panicea (Imbs and Rodkina 2004). The high sensitivity of MS coupled with its ability to detect and quantify a wide range of metabolites from complex mixtures (i.e. cellular extracts) makes it well suited for the analysis of the major and minor compounds found in cnidarian and algal cells (Schauer et al. 2005; Viant 2007; Gordon and Leggat 2010; Davy et al. 2012; Lehnert et al. 2014; Hillyer et al. 2016a).

Metabolic profiling has been used to identify the metabolic contribution within the legumemycorrhizal symbiosis (Schliemann et al. 2008; Zhang et al. 2012). The translocation of amino acids and fatty acids from the mycorrhizal fungus, Glomus intraradices, to the legume host, Medicago truncatula, helped to show for the first time that there are clear differences in symbiosis-dependent primary and secondary metabolism of $M$. truncatula roots, leading to the identification of fungus-specific fatty acids, which can now be used as markers of fungal root colonisation (Schliemann et al. 2008). Changes in metabolite profiles were also observed during the early stages of the symbiosis (Zhang et al. 2012). Furthermore, metabolite profiling was used to investigate the metabolic exchange between the ryegrass Lolium perenne and its mutual symbiont, the endophytic Neotyphodium lolii fungus (Rasmussen et al. 2009). The fungus produces alkaloids that help the grass to avoid insect parasitism and vertebrate herbivory, as well as a number of amino acid metabolites that support the host's metabolism (Rasmussen et al. 2009).

Non-targeted, stable isotopic labelling of metabolites can also provide a global perspective on the fates of specific compounds within the metabolic network, as well as provide evidence for the translocation of fixed carbon (Birkemeyer et al. 2005; Shastri and Morgan 2007; Hiller et al. 2010; Burriesci et al. 2012; Creek et al. 2012; Tremblay et al. 2012; Kopp et al. 2014; Hillyer 2016). Furthermore, the combination of metabolite profiling with genome-scale models of metabolism and other "omic" approaches provides a more comprehensive view of cellular physiology and thus provides opportunities to expand our understanding of metabolic networks and stress responses, and to identify genes associated with specific enzymatic and regulatory activities, such as those involved in symbiosis (Phelps et al. 2002; Hines et al. 2007; Baran et 
al. 2009; Bundy et al. 2009; Gordon and Leggat 2010; Chaston and Douglas 2012; Meyer and Weis 2012; Lehnert et al. 2014; Pernice and Levy 2014). Indeed, metabolite profiling and quantitative PCR were combined to produce a schematic representation of the metabolic network within the ryegrass L. perenne - N. lolii symbiosis (Rasmussen et al. 2009). Meanwhile, the integration of metabolomic and proteomic data recently supported the draft metabolic network reconstruction of Chlamydomonas reinhardtii (May et al. 2008). Such integrated approaches have the ability to identify not only new enzymatic activities, but also new regulatory effects, as shown in Arabidopsis thaliana (Hirai et al. 2004; Hirai et al. 2005). Through these integrated data sets, an improved understanding of the underlying biology is obtained, to be better able to predict a response variable and to gain further insight into mechanistic aspects of the system (Kamburov et al. 2011; Cavill et al. 2015).

\subsubsection{Application to the study of symbiont switching}

The application of metabolomics to the study of the cnidarian-dinoflagellate symbiosis is receiving increased attention (Gordon and Leggat 2010), and methods for the extraction and analysis of metabolites using GC-MS, liquid chromatography-mass spectrometry (LC-MS), and nuclear magnetic resonance (NMR) technologies are emerging (Gordon et al. 2013). Studies include characterisation of the changes in metabolite profiles and mobile products of Aiptasia when in symbiosis (Burriesci et al. 2012), and how the metabolite profiles of Symbiodinium vary between types (Klueter et al. 2015). Metabolomics-based techniques have also been applied to investigate the effects of environmental perturbations on the metabolite profiles of the symbiotic partners, including thermal stress in Aiptasia (Hillyer et al. 2016a) and in the coral Acropora aspera (Hillyer et al. 2016b), under both increased temperature and ocean acidification in Pocillopora damicornis (Sogin et al. 2016), and the effect of nutrient enrichment on the secondary metabolite composition of the soft coral Sarcophyton ehrenbergi (Fleury et al. 2000).

Major gaps still remain in our understanding of the implications elicited to central metabolism and homeostatic pathways in both the host and symbiont as a result of switching partners. Determining the nutritional implications at the individual metabolite level will help to elucidate the specific physiological costs associated with certain symbiont types. Metabolomics-based techniques provide an excellent platform to investigate the variation in metabolite contribution by different symbiont types, and the role of metabolite exchange in symbiosis specificity, to uncover the adaptive potential of the association. 


\subsection{Aims and objectives}

The overall aim of this thesis was to establish what role, if any, nutritional transfer determines host-symbiont specificity in the cnidarian-dinoflagellate symbiosis. To address this aim, this study had four specific objectives. The first three objectives employed the model symbiotic cnidarian Aiptasia, while the final objective extended the study to a reef coral. These objectives were:

1. To optimise and evaluate the potential of menthol incubations for producing aposymbiotic cnidarian hosts, so facilitating research on symbiosis establishment.

The hypotheses were: a) menthol will produce aposymbiotic anemones at a faster rate than a more traditional cold-shock treatment; b) the ability for the anemone hosts to re-establish a successful symbiosis will be unaffected by menthol treatment; and c) physiological function of the symbiosis in re-infected anemones will be unaffected by menthol treatment.

2. To determine the gene-to-metabolite pathways involved in the successful establishment of the cnidarian-dinoflagellate symbiosis, by integrating and comparing the metabolite and gene-expression profiles of a homologous (i.e. naturally-occurring symbiont) versus heterologous symbiotic association.

The hypotheses were: a) there will be differences in the infectivity and autotrophic potential of the different symbiotic associations; b) symbiont type and state would impact the geneexpression profile of the anemone host; c) symbiont type and state would impact the metabolite profile of the anemone host; d) symbiont type and state would impact the molecular processes of the anemone host, and hence the function and potential viability of the symbiosis.

3. To identify and quantify translocated metabolites in different host-symbiont pairings and relate patterns of translocation to symbiont infectivity, using stable isotopic labelling.

The hypotheses were: a) the identity and quantity of translocated metabolites differs in the presence of different symbiont types; b) a shift in the incorporation of translocated carbon into downstream pathways will occur as a result.

4. To determine the effect of symbiont type on the metabolite profile of both the host and symbiont in the field, in a coral species that naturally contains different symbiont types.

The hypothesis was: host and symbiont metabolite profiles will differ in response to the composition of the resident homologous Symbiodinium population. 


\section{CHAPTER 2}

\section{Menthol-induced bleaching rapidly and effectively provides experimental aposymbiotic sea anemones (Aiptasia sp.) for symbiosis investigations}

\subsection{Introduction}

The symbiosis between corals and dinoflagellate algae of the genus Symbiodinium promotes the success of coral reefs in nutrient-poor tropical seas. However, there are still large gaps in our knowledge about how this symbiosis is established and maintained (Weis et al. 2008; Weis and Allemand 2009; Davy et al. 2012). Moreover, we do not fully understand how and why this symbiosis breaks down under stress (i.e. bleaching) (Weis 2008). These knowledge gaps hinder our capacity to understand the function of coral reefs, and to predict how coral reefs might respond to our changing environment.

In order to elucidate the cellular basis of the coral-dinoflagellate relationship, the symbiotic sea anemone Aiptasia sp. has been widely adopted as a model system due to its robust nature and the ability to produce large populations easily in the laboratory (Weis et al. 2008). Furthermore, Aiptasia sp. can be cleared of symbionts, maintained in an aposymbiotic state for extended periods, and successfully re-infected with a variety of Symbiodinium types (Schoenberg and Trench 1980b; Belda-Baillie et al. 2002; Hawkins and Davy 2012; Starzak et al. 2014). The ease of experimental manipulation combined with the rapidly increasing resolution of genomic, proteomic and metabolic databases for Aiptasia sp., makes this symbiotic anthozoan an ideal candidate for biochemical and genetic experiments that will further our understanding of the cellular processes underlying the cnidarian-dinoflagellate symbiosis (Weis et al. 2008; Sunagawa et al. 2009; Peng et al. 2010; Lehnert et al. 2012).

Currently, the most commonly used method to render anemones (including Aiptasia sp.) aposymbiotic, combines temperature stress (heat- or cold-shock), followed by dark treatment and/or chemical inhibition of photosynthesis with 3-(3,4-dichlorophenyl)-1,1-dimethylurea (DCMU) (Muscatine et al. 1991; Wang and Douglas 1998; Sawyer and Muscatine 2001; Belda-Baillie et al. 2002; Kuo et al. 2004; Hawkins and Davy 2012; Xiang et al. 2013; Lehnert et al. 2014; Starzak et al. 2014). This is a slow, laborious process which often requires months of preparation (Xiang et al. 2013; Starzak et al. 2014), and may not result in the full eradication of in hospite endosymbionts (Schoenberg and Trench 1980b; Wang and Douglas 1998; BeldaBaillie et al. 2002). 
Recently, Wang et al. (2012) described a method of menthol-induced bleaching in the corals Isopora palifera and Stylophora pistillata. Menthol is known to cause local anaesthetic effects in neuronal and skeletal muscles via blocking voltage-operated sodium channels (Haeseler et al. 2002). This cellular response has led to its use as a marine anaesthetic (Alexander 1964; Gusmão 2010; Lauretta et al. 2014). Menthol is known to act on a variety of different membrane receptors, including transient receptor potential (TRP)M8 that results in an increase in intracellular $\mathrm{Ca}^{2+}$ concentrations and causes a cold sensation in vertebrates (Okazawa et al. 2000; McKemy et al. 2002; Peier et al. 2002; Reid et al. 2002; Hans et al. 2012). As proposed by Wang et al. (2012), the mechanism of menthol-induced bleaching might be attributable to $\mathrm{Ca}^{2+}$ stimulated exocytosis (Pang and Südhof 2010). Preliminary experiments conducted by Wang et al. (2012) also suggest that menthol inhibition of Symbiodinium photosystem II activity may play a role in the expulsion of the algal cells or the digestion of the Symbiodinium cells by the host. Although the exact mechanism by which menthol induces symbiont expulsion is not yet clear, the findings of Wang and co-authors provide a platform for developing the use of menthol to generate aposymbiotic Aiptasia sp.

Therefore, the applicability of this approach to Aiptasia sp. was tested, in terms of its effectiveness and impact on the capacity for symbiosis reestablishment. In particular, it was determined whether menthol can rapidly and effectively produce aposymbiotic anemones, and whether these anemones can be experimentally re-infected with symbiotic dinoflagellates to similar cell densities as untreated and healthy symbiotic anemones. Ultimately, a method to assist the many researchers around the world who use the Aiptasia model system to further our understanding of the coral-dinoflagellate symbiosis is described.

\subsection{Materials and Methods}

\subsubsection{Experimental organisms}

Symbiotic individuals of Aiptasia sp. $(n=300)$ were harvested from a long-term lab stock maintained in $1 \mu \mathrm{m}$ filtered seawater (FSW) at $25^{\circ} \mathrm{C}$, with light provided by AQUA-GLO T8 fluorescent bulbs at $\sim 95 \mu \mathrm{mol}$ photons $\mathrm{m}^{-2} \mathrm{~s}^{-1}$ (light:dark $=12 \mathrm{~h}: 12 \mathrm{~h}$ ). Anemones were maintained in $1 \mu \mathrm{m}$ filtered seawater (FSW) aerated with a Hailea ${ }^{\circledR}$ aquarium air pump at 2 $\mathrm{L} / \mathrm{min}$ and at $25^{\circ} \mathrm{C}$ using a WEIPRO ${ }^{\circledR}$ Temperature Controller MX-1019. These lighting and aquarium conditions were maintained throughout the 19-week experiment. The anemones were 
evenly divided among the treatments (menthol, cold shock, and untreated) and allowed to settle for $72 \mathrm{~h}$ before beginning a 4-week treatment period.

The Symbiodinium culture (ID: FLAp2) was grown at $25^{\circ} \mathrm{C}$ and an irradiance of $100 \mu \mathrm{mol}$ photons $\mathrm{m}^{-2} \mathrm{~s}^{-1}$ on a 12:12 h light:dark cycle. Symbiodinium cells were sub-cultured from a laboratory stock and grown in silica-free $f / 2$ medium (AusAqua PtyLtd, SA, Australia) for six weeks before use in the infection study. To genotype the Symbiodinium cells, DNA was extracted as described by Hill et al. (2014) from an algal pellet derived from a $1 \mathrm{~mL}$ aliquot of the Symbiodinium suspension. Samples were immediately used for PCR, using the thermal cycling regime and reaction mixture of Hill et al. (2014), and the outer primers ITSintfor2 and ITS2Rev2. PCR products were cleaned with ExoSAP-IT (USB Corporation, OH, USA) and sequenced by the Macrogen Sequencing Service (Macrogen Inc., Seoul, South Korea). Sequences were aligned with Geneious v. 7.0 (Biomatters Ltd., Auckland, NZ) and a BLAST search carried out against Symbiodinium ITS2 sequences in GenBank. Using this approach, Symbiodinium genotype was confirmed as B1.

\subsubsection{Menthol versus cold-shock treatment}

Menthol (20\% w/v in ethanol; Sigma-Aldrich, Auckland, NZ) was added to $1 \mu \mathrm{m} \mathrm{FSW} \mathrm{at} \mathrm{a}$ final concentration of $0.19 \mathrm{mM}$. This concentration resulted in successful bleaching without causing mortality. A higher concentration of $0.38 \mathrm{mM}$ as suggested by Wang et al. (2012) was trialled prior to this experiment, but caused mortality of over $50 \%$ of the anemones (unpublished data). The anemones were incubated in the menthol/FSW solution for $8 \mathrm{~h}$ after which the menthol/FSW was removed and the anemones incubated in $1 \mu \mathrm{m}$ FSW for $16 \mathrm{~h}$. DCMU (100 mM dissolved in ethanol, Sigma-Aldrich, Auckland, NZ) was added to a final concentration of $5 \mu \mathrm{M}$, in order to prevent nuisance algal blooms and to limit the reestablishment of any residual symbiont cells by inhibiting photosynthesis. This 24 -h cycle was repeated for four consecutive days. The 8 -h menthol/FSW incubation occurred during the 12-h light period. The 4-day treatment was repeated after a 3-day break, during which anemones were maintained under the lighting and temperature conditions stated above, with biweekly feeding with Artemia sp. nauplii.

For cold shock, the water was replaced with $4^{\circ} \mathrm{C} 1 \mu \mathrm{m}-\mathrm{FSW}$, and placed in a refrigerator at $4^{\circ} \mathrm{C}$ for $4 \mathrm{~h}$. Afterwards, the water was replaced with $25^{\circ} \mathrm{C} 1 \mu \mathrm{m}$-FSW containing a final concentration of $50 \mu \mathrm{M}$ DCMU (100 mM dissolved in EtOH, Sigma-Aldrich, Auckland, NZ) and held under the lighting and aquarium conditions described above. The anemones were 
incubated under these conditions for three days, and the treatment then repeated. The anemones were unfed for the duration of the cold-shock, as per the protocol described by (Steen and Muscatine 1987). Anemones were cold-shocked a total of eight times (twice per week) during the treatment period.

After the 4-week treatment period, all anemones were maintained in $1 \mu \mathrm{m}-\mathrm{FSW}$ at $25^{\circ} \mathrm{C}$ under the irradiance regime described above, with biweekly feeding, for an additional 15 weeks. Their symbiotic status was assessed via confocal fluorescence microscopy and cell density estimates (described below) at Weeks 1-5, 7, 9, 11, and 19.

The untreated group of anemones was kept under the same thermal and light regimes as the menthol-treated and cold-shocked anemones, with biweekly feeding throughout the entire 19week experiment. They were assessed for symbiont density at the same time-points as the experimental anemones, to obtain an average symbiont density of untreated, healthy anemones.

\subsubsection{Reinfection of aposymbiotic anemones}

The capacity to re-infect menthol-treated anemones with algal symbionts was tested; this was not possible with cold-shocked anemones since they were not fully aposymbiotic. Anemones were infected with cultured homologous Symbiodinium type B1, i.e. symbionts originally isolated from Aiptasia sp. A drop of concentrated Symbiodinium suspension ( 3 x $10^{6}$ cells mL

${ }^{1}$ ) was pipetted onto the oral disc of each anemone, followed by a drop of Artemia sp. nauplii to invoke a feeding response and encourage Symbiodinium uptake (Davy et al. 1997). This process was repeated $4 \mathrm{~h}$ later, and then the water was replaced with $1 \mu \mathrm{m}$ FSW $8 \mathrm{~h}$ after the second infection.

\subsubsection{Confocal microscopy}

Whole anemones from each treatment were examined at the same time-points as cell density assessments by confocal microscopy (Olympus Provis AX70, at 100× magnification) to detect the chlorophyll autofluorescence of any dinoflagellates. Anemones were selected at random from each treatment, and placed in a "relaxation solution" [50\% $1 \mu \mathrm{m} \mathrm{FSW}, 50 \% 0.37 \mathrm{M}$ magnesium chloride $\left(\mathrm{MgCl}_{2}\right)$ ] for 15 min before being moved to a FluoroDish ${ }^{\mathrm{TM}}$ glass-bottom confocal dish (World Precision Instruments, Florida USA). The auto-fluorescence emission of the Symbiodinium cells was excited using a $559 \mathrm{~nm}$ laser and captured at $647 \mathrm{~nm} \pm 10 \mathrm{~nm}$. This was performed to check the rate of bleaching and the rate of reinfection, as well as to detect any background Symbiodinium populations in the post-treatment anemones. 


\subsubsection{Symbiont density and protein content}

Symbiodinium populations were quantified with a haemocytometer and light microscope (100 $\times$ magnification; $n=8$ replicate counts per sample) using whole anemone homogenate $(n=5)$. Cell density was normalised to soluble protein content, measured via the Bradford assay (Bradford, 1976). Cell density was measured at Weeks 1-5, 7, 9, 11, and 19.

\subsection{6. $\mathrm{O}_{2}$ flux}

Fifteen weeks after reinfection, maximum photosynthetic and dark respiratory $\mathrm{O}_{2}$ fluxes were measured to compare re-infected menthol-treated anemones against untreated anemones $(n=$ 4). A FIBOX 3 fibre optic oxygen transmitter and oxygen probe (PreSens GmbH, Germany) were used as described by Starzak et al. (2014). The respiration rate $\left(\mathrm{mL} \mathrm{O}_{2} \mathrm{~h}^{-1}\right)$ was measured for $30 \mathrm{~min}$ in the dark, after which time the rate of net photosynthesis was measured for $30 \mathrm{~min}$ at an irradiance of $\sim 100 \mu \mathrm{mol}$ photons $\mathrm{m}^{-2} \mathrm{~s}^{-1}$. Net photosynthesis and respiration were corrected for background levels using a $1 \mu \mathrm{m}$ FSW-only control, and gross photosynthesis was calculated by the addition of net photosynthesis to dark respiration (Muscatine et al. 1981). The average ratio of gross photosynthesis to respiration (P:R) for 24 hours was calculated for each anemone, by assuming light:dark $=12 \mathrm{~h}: 12 \mathrm{~h}$ and that respiration was constant in the light and dark, and the average ratio for each treatment calculated.

\subsubsection{Statistical tests}

The nonparametric Mann-Whitney $U$ test was used to test for differences between the treatments with respect to symbiont density (SPSS statistical software, v. 20, IBM Corp). Separate analyses were run for the bleaching and post-bleaching periods.

A one-way analysis of variance (ANOVA) was performed on the photosynthetic rate, respiration rate, and log-transformed $\mathrm{P}: \mathrm{R}$ values to determine whether significant differences were present between the re-infected menthol-treated and untreated symbiotic anemones at the end of the 19-week experimental period.

\subsection{Results and Discussion}

\subsubsection{Menthol versus cold shock treatment}

Menthol treatment induced $97-100 \%$ symbiont loss within $14-28$ days, with $60 \%$ of anemones losing all symbionts. In comparison, cold shock never induced $100 \%$ loss within the same time 
frame (Figure 2.1A,B), and any residual symbionts in the menthol- treated anemones did not re-establish a full symbiosis once returned to normal seawater conditions over the course of the experiment (Figure 2.1A,C).

Although statistically, the patterns of symbiont loss during weeks 1-4 were not different between the menthol and cold-shock treatments (Mann-Whitney $U=113, \mathrm{df}=4, p=0.423, n$ $=5$; Figure 2.1A), only the menthol-treated anemones successfully maintained aposymbiotic status in the longer term. In both treatments, symbiont density remained near zero during the first week of recovery (weeks 4-5; Figure 2.1A); however, subsequently there was a significant difference between the cell densities of menthol- and cold-shock-treated anemones (MannWhitney $U=35$, df $=4, p<0.0005 ; n=5$ ), with the menthol-treated anemones remaining mostly symbiont-free $(0-0.05 \%$ of pre-bleaching density) and the cold-shocked anemones becoming fully re-populated (weeks 5-9; Figure 2.1A). Confocal microscopy confirmed these measurements (Figure 2.1C,D). Menthol treatment therefore has considerable potential for the rapid and effective generation of aposymbiotic Aiptasia sp.

\subsubsection{Reinfection of aposymbiotic anemones}

One key use for aposymbiotic Aiptasia sp. is for experimental work that involves re-infection with symbiotic algae of different types and from different host species. It was therefore important to test the infectivity of the menthol-treated anemones, and establish whether there were any significant long-term impacts of menthol treatment versus no treatment on physiological performance (photosynthesis and respiration) of the re-infected anemones. When infected with Symbiodinium cells (ITS2 type B1), symbiont density increased slowly during the initial 2 weeks, followed by a rapid increase during weeks 2-12 (Figure 2.2A). Confocal microscopy confirmed that menthol- treated anemones that were infected with Symbiodinium were fully repopulated after 12 weeks (Figure 2.2B), while those that were not infected remained aposymbiotic (Figure 2.2C). The infection pattern and maximum cell density were similar to those of other re-infection studies using heat- and cold-shock bleached Aiptasia and type B1 Symbiodinium, where a steep increase in cell density was observed in the first 4 weeks, followed by stabilisation (Belda-Baillie et al., 2002; Schoenberg and Trench, 1980; Starzak et al., 2014). Additionally, the photosynthetic rate $(p=0.432 ; n=4)$, respiration rate $(p=0.347 ; n$ $=4)$ and ratio of photosynthesis to respiration $(p=0.368 ; n=4)$ in these anemones were similar to those of untreated symbiotic anemones. This indicates that their capacity to re- form a functional symbiosis was not compromised, further highlighting the value of this method. 
As expected, given its anaesthetic properties (Alexander, 1964; Lauretta et al., 2014), menthol induced tentacle relaxation and unresponsiveness in anemones for the duration of the treatment. Once the seawater was replaced with regular $1 \mu \mathrm{m}-\mathrm{FSW}$, the anemones were able to regain full movement and feeding capabilities within $\sim 15 \mathrm{~min}$. This is in stark contrast to the residual effects of cold shock on anemones, where long-lasting tentacle retraction and occasional mortality were observed.
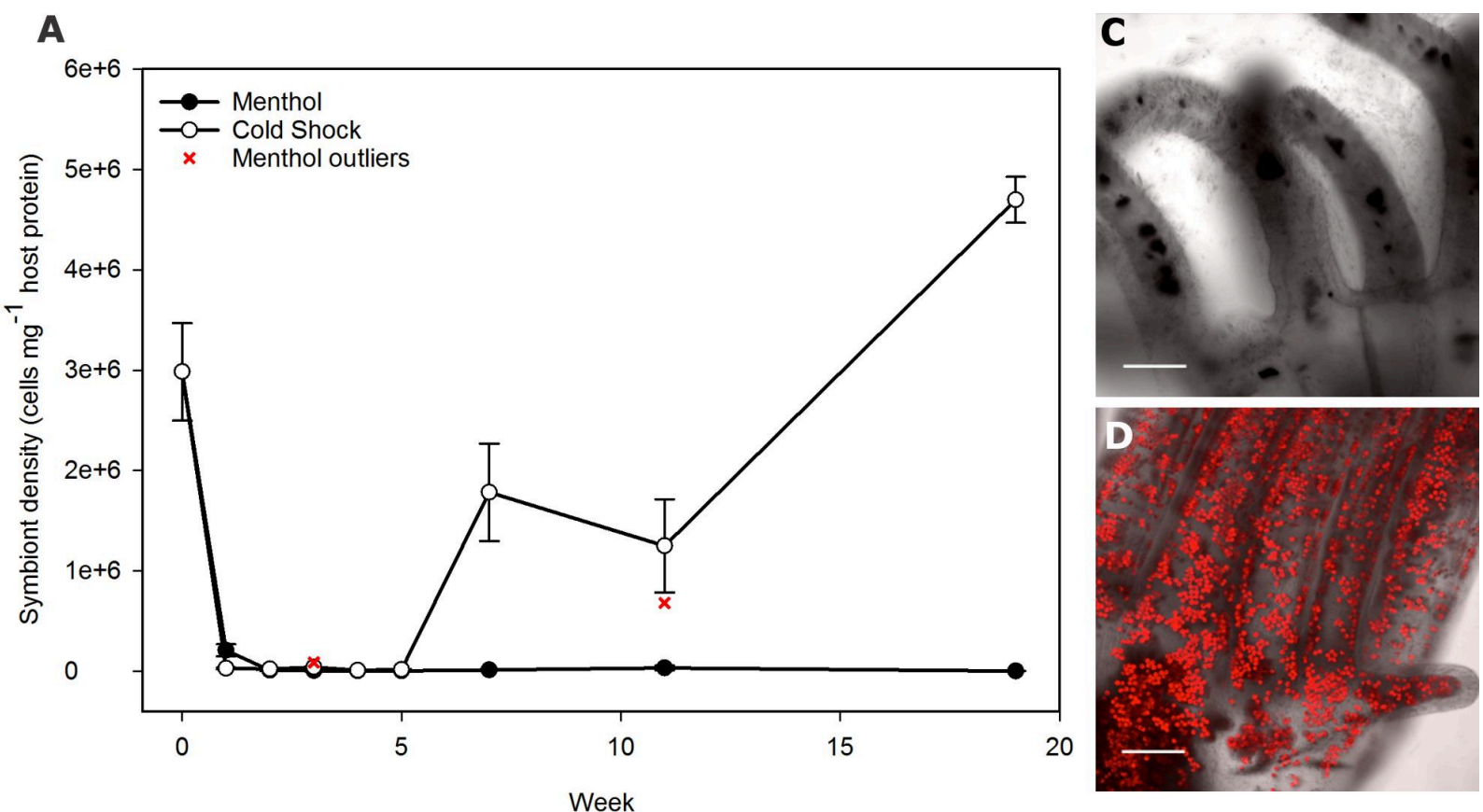

B

0 1 2
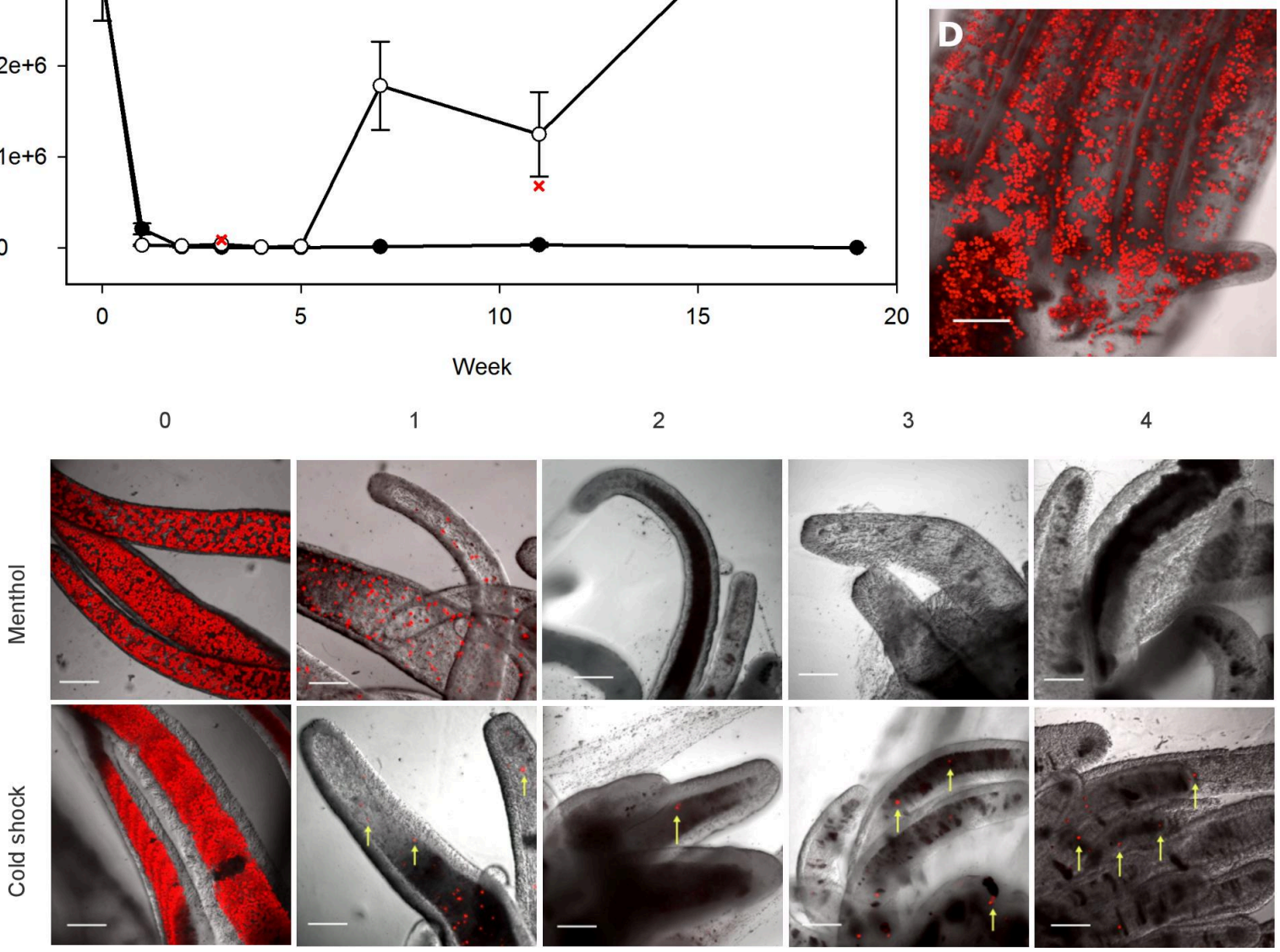

3

4

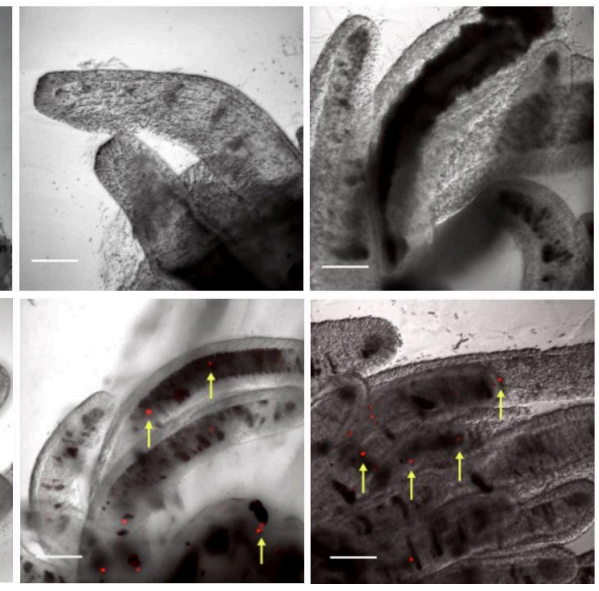

Figure 2.1 Effects of menthol and cold shock on algal cell density in Aiptasia sp. (A) Density of Symbiodinium cells in menthol-treated (closed circles) and cold-shocked (open circles) anemones over a 19-week period. The anemones were treated for the first four weeks, then left to recover in regular FSW for the remaining 15 weeks. Outliers (defined as exceeding $1.5 \times$ IQR) were detected in the menthol-treated anemones at Weeks 4 and 11, and are indicated by a red cross. Values are mean \pm S.E.M.; $n=5$. (B) Confocal microscopy images of anemones during menthol treatment and cold shock (Weeks 1-4). Yellow arrows indicate Symbiodinium cells in host tissues. (C \& D) Confocal fluorescence images of menthol-treated and coldshocked anemones, respectively, 15 weeks post-treatment (i.e. Week 19). Scale bars are 100 $\mu \mathrm{m}$. 

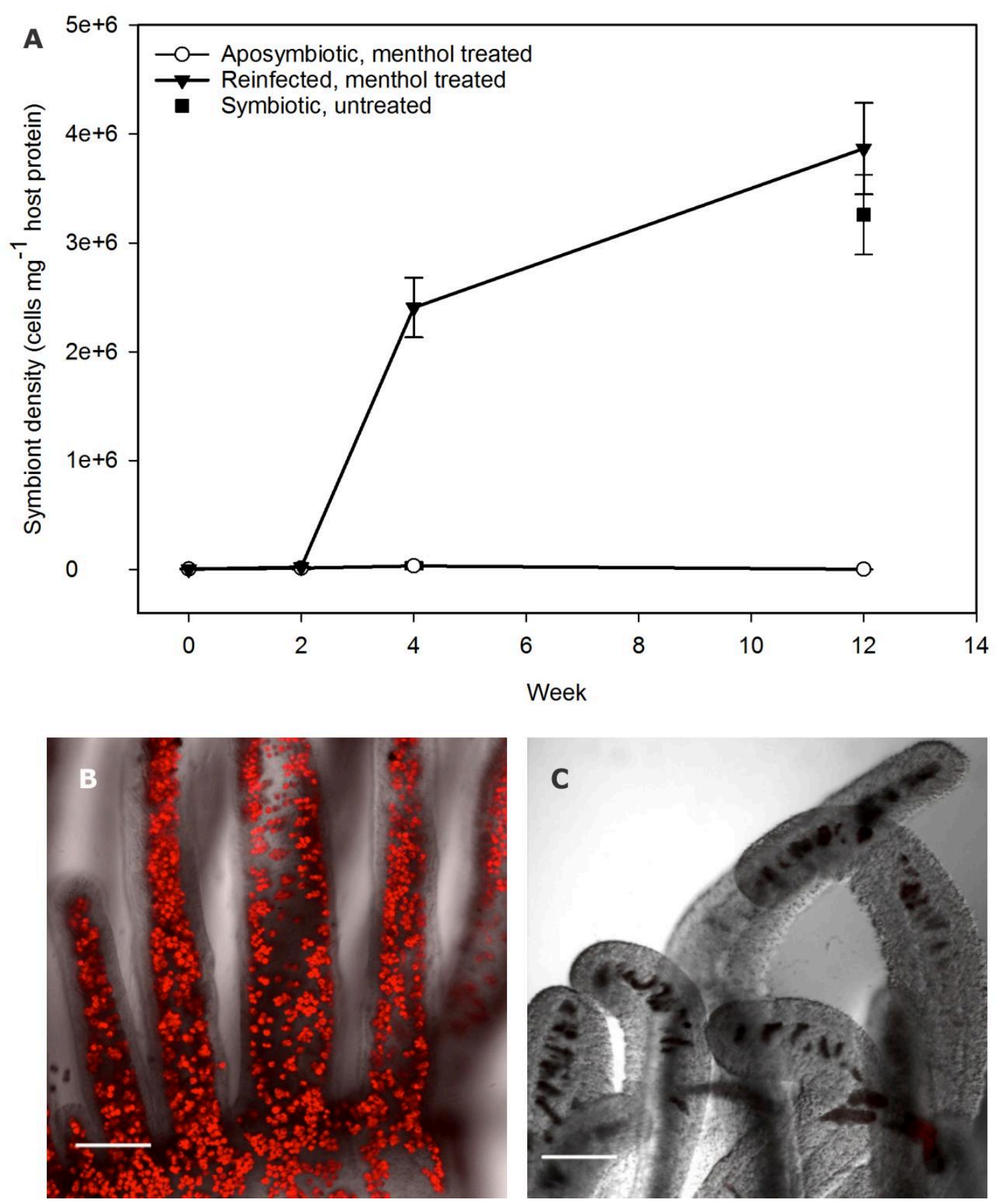

Figure 2.2 Repopulation of menthol-treated Aiptasia sp. with algal symbionts. (A) Symbiont cell density over a 12-week period after anemones were experimentally re-infected with the homologous Symbiodinium type B1 (closed triangles). Also shown are symbiont densities in symbiotic, untreated anemones (closed square) and menthol-treated anemones that were not experimentally re-infected (open circles). Values are mean \pm S.E.M.; $n=5$. (B \& C) Confocal images showing, respectively, the re-established symbiont population 12 weeks after reinfection versus tissues in a non-infected aposymbiotic anemone. Scale bars are $100 \mu \mathrm{m}$.

\subsection{Conclusion}

In summary, I provide a rapid and effective method for the generation of aposymbiotic Aiptasia sp. that will facilitate studies of symbiosis establishment and maintenance, host- 
symbiont recognition, and symbiosis function with this model system. In particular, menthol treatment has the potential to rapidly create large stocks of aposymbiotic anemones without compromising their infectivity. Ultimately, this method will help to accelerate the rate of discovery as we try to better understand the function of coral reefs, and their response and adaptive capacity in the face of climate change. 


\section{CHAPTER 3}

\section{Integrated pathway analysis of transcriptomic and metabolomic data suggests that optimal nutritional exchange and redox homeostasis operate in partner specificity in the cnidarian-dinoflagellate symbiosis}

\subsection{Introduction}

Reef-building corals are dependent on a symbiosis with a highly diverse group of photosynthetic dinoflagellates of the genus Symbiodinium. These dinoflagellate symbionts are housed within a membrane of animal origin (called the symbiosome) in the gastrodermal cells of the host coral (Wakefield and Kempf 2001). Bi-directional exchange of organic and inorganic compounds between the partners is central to the ecological success and stability of the coral-dinoflagellate symbiosis (Muscatine and Hand 1958; Muscatine and Cernichiari 1969; Davy et al. 2012), and is critically important to the construction and persistence of coral reefs ecosystems (Muller-Parker and D'Elia 1997; Moberg and Folke 1999). The algal symbiont receives dissolved inorganic nutrients (carbon, nitrogen and phosphorus) from the host, and may also receive host-derived amino acids, lipids and fatty acids (Wang and Douglas 1999; Imbs et al. 2014). In return, symbionts translocate organic products of carbon fixation and nitrogen assimilation to the host, including sugars, sugar alcohols, amino acids and lipids (Gordon and Leggat 2010; Kopp et al. 2015). Despite being a topic of research for decades, the quality and quantity of photosynthate that is translocated from symbiont to host are not fully understood (Venn et al. 2008; Gordon and Leggat 2010; Burriesci et al. 2012; Davy et al. 2012; Kopp et al. 2015; Hillyer et al. 2016a), however most studies to date point to glucose as the major translocated photosynthetic product in the symbiosis (Whitehead and Douglas 2003; Burriesci et al. 2012; Hillyer et al. 2016a).

Disruption of the symbiosis (dysbiosis) due to the loss of Symbiodinium cells from coral tissues (known as coral bleaching) is primarily due to increasing sea surface temperatures from global warming, and is occurring with increasing frequency and severity. Bleaching therefore poses a major threat to the future of coral reefs (Hoegh-Guldberg et al. 2011). If bleaching stress is not lethal, however, it may present an opportunity for the Symbiodinium community resident within a host to 'shuffle' to an alternative dominant type (i.e. where a mixed population is already present inside the host) or 'switch' to a new type acquired from the surrounding environment (Buddemeier and Fautin 1993; Fautin and Buddemeier 2004; Berkelmans and 
Van Oppen 2006; Stat and Gates 2011; Boulotte et al. 2016). These putative mechanisms would rely on the considerable molecular and physiological diversity within the genus Symbiodinium, with nine clades (A-I) and numerous sub-clades (types) described to date (Pochon and Gates 2010). Physiological diversity in Symbiodinium has been demonstrated at the cladal and type levels including differences in photosynthetic carbon translocation and in tolerance to stress (Loram et al. 2007; Starzak et al. 2014; Leal et al. 2015). Consequently, a single host species has been shown to display different physiologies depending on the Symbiodinium type it hosts (Rowan 2004; Goulet et al. 2005; Berkelmans and Van Oppen 2006; DeSalvo et al. 2010b). Changes in Symbiodinium communities in favour of symbionts physiologically suited to a prevailing environmental condition have the potential to affect the ability of the holobiont to withstand changing ocean conditions, such as elevated sea surface temperatures (Buddemeier and Fautin 1993; Little et al. 2004; Berkelmans and Van Oppen 2006).

Despite the adaptive potential of shuffling/switching symbiont types, corals typically form symbiotic relationships with specific Symbiodinium genotypes (Baker 2003; Goulet 2006). Recently, high-resolution molecular techniques identified symbiont types acquired anew from the surrounding seawater in the background communities of adult corals following bleaching, suggesting that there is the potential to form stable symbioses with exogenous algal symbionts (Boulotte et al. 2016). Symbiosis establishment between a host cnidarian and dinoflagellate from the surrounding environment is thought to include mechanisms of inter-partner recognition also referred to as winnowing, whereby only compatible symbionts are retained within the host cells (Nyholm and McFall-Ngai 2004; Wood - Charlson et al. 2006; Kvennefors et al. 2008; Dunn and Weis 2009; Davy et al. 2012). Once established, the mechanisms responsible for the dynamic regulation and persistence of the symbiosis likely involves the modulation of the host immune-response (Voolstra et al. 2009; Schnitzler and Weis 2010; Mohamed et al. 2016) and nutritional flux between the two partners (Loram et al. 2007). In particular, different symbiont strains may release different types and quantities of photosynthetic products to the host and hence influence the nutritional potential of the symbiosis and its overall fitness (Hohman et al. 1982; Loram et al. 2007; Starzak et al. 2014; Leal et al. 2015). Investigating the cellular and metabolic processes determining the success of a novel symbiosis in the cnidarian-dinoflagellate symbiosis may help to elucidate the capacity of coral reefs to withstand climate change.

Combining data collected using high-throughput 'omics' techniques is a novel approach for the study of cnidarian-dinoflagellate symbiosis. Transcriptomics studies have provided valuable 
insight into the intimate molecular mechanisms involved in the coral-dinoflagellate symbiosis, including studies comparing gene expression in symbiotic versus aposymbiotic hosts, responses to thermal stress, and exposure to coral diseases (DeSalvo et al. 2010a; Ganot et al. 2011; Leggat et al. 2011b; Meyer et al. 2011; Meyer and Weis 2012; Barshis et al. 2013; Barshis et al. 2014; Lehnert et al. 2014; Wright et al. 2015; Mohamed et al. 2016). However, annotated transcriptomic data for cnidarians are still limited, and the function of many genes identified in cnidarian-dinoflagellate symbiosis is still unresolved (Lehnert et al. 2012; Lehnert et al. 2014; Parkinson et al. 2016). Metabolomic analyses reveal a set of metabolites that reflect a phenotype under particular environmental conditions, but alone are insufficient to identify the pathways involved; for example, an increase in abundance may be due to active exchange in the symbiosis or accumulation due to inactive or dysfunctional metabolism and/or exchange (Kamburov et al. 2011). Integration of these datasets will improve our understanding of the molecular mechanisms underlying the regulation of cnidarian-Symbiodinium symbioses, and lead to insights into the benefits of symbiont shuffling/switching as a strategy for coping with changing environmental conditions.

The sea anemone Aiptasia sp. is a widely adopted model system for the cnidariandinoflagellate symbiosis (Weis et al. 2008; Lehnert et al. 2012). It most commonly forms an association with S. minutum (referred to in the study by its ITS2 type B1, (Starzak et al. 2014)) (Weis et al. 2008; Lehnert et al. 2012; Thornhill et al. 2013; Chapter 2). Crucially, Aiptasia can be rendered aposymbiotic and then re-colonised with a range of different symbiont types, making it an ideal system for the study of symbiosis establishment and host-symbiont regulation (Schoenberg and Trench 1980b; Starzak et al. 2014; Chapter 2). In the present study, I used Aiptasia to compare the autotrophic potential of an appropriate (homologous) versus a novel (heterologous) symbiosis, and characterise gene expression and metabolite profiles of the host in response to symbiotic state and symbiont type. In particular, I focused on the heterologous symbiont $S$. trenchii (referred to in the study by its ITS2 type D1a), because it frequently populates corals that have recovered from bleaching (Stat and Gates 2011; Kemp et al. 2014; Boulotte et al. 2016). This has been attributed to its ability to associate with a variety of coral hosts and its relative thermal tolerance, although this is not a ubiquitous response (Thornhill et al. 2009; Stat and Gates 2011). The potential of D1a to provide resilience and assist in host recovery makes it an especially interesting candidate for investigations into the molecular and physiological bases of the symbiosis (Berkelmans and Van Oppen 2006; Stat and Gates 2011). 
Next generation RNA-seq transcriptomic analysis and Gas Chromatography-Mass Spectrometry (GC-MS) metabolite profiling were conducted on the host tissues of Aiptasia in either aposymbiotic, homologous, or heterologous symbiotic states. Individual and integrated pathway analyses of the transcriptomic and metabolomics data were examined. I expected to find that symbiont type affects the infectivity and molecular response of the host. Investigating the significantly affected pathways further unravelled mechanisms of symbiosis specificity in the cnidarian-dinoflagellate symbiosis. Through the novel integration of the multiple 'omics' techniques, I demonstrate that optimal nutrient availability and oxidative stress tolerance are important biological mechanisms in the formation of novel symbioses.

\subsection{Materials and Methods}

\subsubsection{Experimental organisms}

Symbiotic Aiptasia $(n \sim 700)$, originally collected from Hawaii were harvested from a longterm laboratory stock and maintained in $0.22 \mu \mathrm{m}$ aerated filtered seawater (FSW). Temperature was maintained at $25{ }^{\circ} \mathrm{C}$ with light provided by AQUA-GLO T8 fluorescent bulbs at $\sim 95 \mu \mathrm{mol}$ photons $\mathrm{m}^{-2} \mathrm{~s}^{-1}$ (light:dark $=12 \mathrm{~h}: 12 \mathrm{~h}$ ). Anemones were fed twice a week with freshly hatched Artemia sp. nauplii.

Anemones were rendered aposymbiotic following the menthol-bleaching procedure (Chapter 2). Whole anemones $(n=20)$ from the bleaching treatment were examined by confocal microscopy (Olympus Provis AX70, at 100× magnification) to detect the chlorophyll autofluorescence of any residual dinoflagellates. Anemones were selected at random and placed in a 'relaxation solution' [ $50 \% 0.22 \mu \mathrm{m}$ FSW, 50\% $0.37 \mathrm{~mol}^{-1}$ magnesium chloride $\left(\mathrm{MgCl}_{2}\right)$ ] for $15 \mathrm{~min}$ before being moved to a FluoroDishTM glass-bottom confocal dish (World Precision Instruments, FL, USA). The autofluorescence emission of the Symbiodinium cells was excited using a $559 \mathrm{~nm}$ laser and captured at $647 \pm 10 \mathrm{~nm}$. This confirmed the aposymbiotic state of the anemones.

Two different Symbiodinium cultures were used, comprising of the homologous type $S$. minutum (ITS2 type B1) originally isolated from laboratory Aiptasia stock, and a heterologous type S. trenchii (ITS2 type D1a, culture I.D. Ap2). Symbiodinium cells were sub-cultured from a laboratory stock and grown in silica-free F/2 medium (AusAqua Pty, SA, Australia) for 6 weeks before use in the colonisation study. Cultures were grown at $25{ }^{\circ} \mathrm{C}$ and light provided by 
cool white fluorescent lamps (Phillips, 18W 840), at an irradiance of $100 \mu \mathrm{mol}$ photons $\mathrm{m}^{-2} \mathrm{~s}^{-1}$ with a $12 \mathrm{~h}: 12 \mathrm{~h}$ light:dark photoperiod.

\subsubsection{Inoculation of aposymbiotic Aiptasia with Symbiodinium}

Aposymbiotic anemones $(n \sim 220$ per treatment) were starved for seven days prior to inoculation. One drop of Symbiodinium cells at $\sim 3 \times 10^{6}$ cells $\mathrm{mL}^{-1}$ was placed onto the oral disc of each anemone (except for the aposymbiotic treatment where a drop of $0.22 \mu \mathrm{m}$ FSW only was used), followed by a dilute suspension of Artemia sp. nauplii to enhance phagocytosis (Davy et al. 1997a). The aposymbiotic anemones were exposed to Symbiodinium cells for $24 \mathrm{~h}$ before the FSW was changed. This colonisation procedure was repeated for 3 more weeks (i.e. 4 weeks in total), during which the temperature and light regimes were maintained as per precolonisation, however the anemones were only fed during the symbiont colonisation procedure so as to encourage symbiont colonisation.

\subsubsection{Measurement of $\mathrm{O}_{2}$ flux}

On the sampling day, maximum photosynthetic and dark respiratory $\mathrm{O}_{2}$ fluxes were measured to compare the symbiosis functionality of anemones colonised by homologous type B1 and heterologous type D1a symbionts as per the methods of Starzak et al. (2014). Briefly, individual anemones $(n=4$ per treatment) were placed in a glass chamber of volume $10 \mathrm{~mL}$, itself situated in a glass water bath set at $25^{\circ} \mathrm{C}$. The chamber contained $0.22 \mu \mathrm{m}$ FSW stirred via a magnetic spin bar that rotated the medium speed at which the anemones did not appear stressed. A perforated nylon mesh overlaid the stir bar, on which the anemone was placed and allowed to settle for $1 \mathrm{~h}$ prior to oxygen measurements so that it could attach to the mesh and open its tentacles. The chamber was sealed by a glass lid with a rubber O-ring, into which an oxygen electrode (FIBOX 3 fibre-optic oxygen meter; PreSens GmbH, Germany) and temperature probe were inserted. These were connected to the oxygen meter and were used to measure the respiration rate $\left(\mathrm{R}, \mathrm{mL} \mathrm{O}_{2} \mathrm{~h}^{-1}\right)$ for $30 \mathrm{~min}$ in the dark, by which time a constant rate was observed. The oxygen tension was not allowed to fall below 50\% saturation (Davies 1984). Subsequently, the rate of net photosynthesis was measured for $30 \mathrm{~min}$ at an irradiance of $400 \mu \mathrm{mol}$ photons $\mathrm{m}^{-1} \mathrm{~s}^{-1}$ (light-saturation for photosynthesis (Starzak et al. 2014)). Light was provided by a Thorn PAR (photosynthetically active radiation) $38150 \mathrm{~W}$ sealed beamreflector lamp and irradiance on the surface of the chamber was measure by a QSL-100 irradiance meter (Biospherical Instruments Inc.). Net photosynthesis and respiration were corrected for seawater microbial activity using a FSW-only control, and gross photosynthesis 
( $\left.\mathrm{P}_{\text {gross }}\right)$ was calculated by the addition of net photosynthesis to dark respiration (Muscatine et al. 1981).

After the $\mathrm{O}_{2}$-flux measurements were complete, the individual anemones were then placed in a 1.5-mL microcentrifuge tube and homogenised in $1 \mathrm{~mL}$ FSW using a plastic tissue grinder until fully homogenised, and the homogenate then centrifuged at $1,000 \times g$ for $5 \mathrm{~min}$ to separate the animal and algal fractions. The animal supernatant was collected, and the algal pellet re-suspended in $1 \mathrm{~mL}$ FSW. Symbiodinium cell densities for $\mathrm{O}_{2}$-flux normalisation were quantified from the algal fraction using Improved Neubauer haemocytometer counts (Boeco, Germany) with a minimum of ten replicate counts per sample (i.e. to a confidence interval below $10 \%$ ). Cell density was normalised to soluble protein content of the host fraction, which was assessed by the Bradford assay (Bradford 1976) and bovine-serum albumin (BSA) as the standard. The total gross photosynthetic and respiration rates were adjusted for the respiration chamber volume and normalised to host protein content, while gross photosynthesis was also normalised to algal cell density. The average ratio of gross photosynthesis to respiration $\left(\mathrm{P}_{\text {gross }}: \mathrm{R}\right)$ for $24 \mathrm{~h}$ was calculated for each anemone, by assuming a $12 \mathrm{~h}: 12 \mathrm{~h}$ light:dark cycle and that respiration was constant in the light and dark, providing a proxy for autotrophic potential. Data were assessed for normality and homoscedasticity were assessed using ShapiroWilk and Levene's tests, respectively, and a Student $t$-test performed at the $p<0.05$ probability level to compare $\mathrm{R}, \mathrm{P}_{\text {gross }}$, and $\mathrm{P}_{\text {gross }}: \mathrm{R}$ between treatments, using SPSS statistical software (v. 20, IBM Corp).

\subsubsection{RNA and metabolomics sampling}

Prior to sampling, anemones were starved for one week. For each of the five replicate samples for each treatment, anemones were allocated into transcriptomic ( $n=10$ anemones) and metabolomics ( $n=30$ anemones) subsamples. Samples were taken at midpoint through the light cycle. RNA samples were collected through the addition of $50 \mathrm{~mL}$ of an RNA stabilising buffer (ammonium sulphate; 1M sodium citrate; 0.5 M EDTA; pH 5.2) and left overnight at 4 ${ }^{\circ} \mathrm{C}$ before being stored at $-80{ }^{\circ} \mathrm{C}$. For metabolite profiles, the seawater was gently discarded from each replicate and flash frozen in liquid nitrogen to quench the metabolism $(\sim 10 \mathrm{~s})$. Samples were stored at $-80^{\circ} \mathrm{C}$ until processing.

\subsubsection{RNA extraction}

Total RNA was extracted and purified from individual anemone tissue selected at random from each frozen sample ( $n=3$ individual anemones per sample per treatment) using a combination of the TRIzol® RNA isolation (Life Technologies, CA, USA) and the RNeasy Mini Kit 
(Qiagen, CA, USA) protocols. Extracted RNA was DNase-treated using a TURBO DNA-Free Kit (Ambion, CA, USA) according to the manufacturer's instructions. RNA quantity and quality was assessed using the NanoDrop ND-1000 UV-Vis spectrophotometer (Thermo Scientific, MA, USA) and gel electrophoresis. Samples containing less than $1 \mu \mathrm{g}$ of total RNA were excluded from the analysis. From the remainder, a single extraction with the highest RNA quality was selected per sample per treatment for further analysis ( $n=5$ per treatment).

\subsubsection{Preparation of cDNA for Illumina sequencing}

One $\mu \mathrm{g}$ of total RNA from each sample was heat fragmented at $95{ }^{\circ} \mathrm{C}$ for $10 \mathrm{~min}$. cDNA libraries were synthesized following a $3^{\prime}$ tag based RNA-Seq protocol described previously (Meyer et al. 2011). Individual cDNA libraries were labelled with sequence specific barcode adaptors and final cDNA samples ranging in size from 250-350 bp were excised from a 2\% agarose gel.

\subsubsection{Sequencing, processing and mapping}

cDNA libraries were pooled and sequenced in a single lane on an Illumina HiSeq 2000. Singleend sequencing of 15 cDNA libraries produced reads 100 base pairs in length. Sequences were de-multiplexed based on their sample-specific barcode adaptors. Raw sequences were filtered and trimmed using custom Perl scripts (https://github.com/Eli-Meyer) to remove uninformative (adaptor sequences, homopolymer repeats) or low quality ( $>20$ positions with quality scores $<$ 20) reads. High quality reads were mapped to the reference transcriptome using the software package SHRiMP (v2.2.2) (Rumble et al. 2009; David et al. 2011).

\subsubsection{RNA seq Reference transcriptome assembly, and annotation}

A concatenated transcriptome made up of both Aiptasia pallida and Symbiodinium clade B transcriptomes was assembled for mapping of raw reads. The Symbiodinium transcriptome was included to separate the Symbiodinium genes from Aiptasia genes in the downstream analyses. Details concerning the assembly and annotation of the Symbiodinium clade B portion of the concatenated transcriptome have been previously described by Bayer et al. (2012). The reference transcriptome for $A$. pallida was assembled using raw reads kindly provided by Erik Lehnert (now available at SRA accession SRX231866). Before assembly, the raw reads were processed to remove low quality or uninformative reads using custom Perl scripts (https://github.com/Eli-Meyer). Any reads with $>30$ positions having low quality scores (Phred score $<20$ ), and any reads matching the adaptors used in library construction (cross_match score $>15)$ were removed. The cleaned, high-quality reads were then assembled using the de 
novo transcriptome assembler Trinity v2.0.2 (trinityrnaseq r2012-06-08) with default settings (Grabherr et al. 2011). The assembly was annotated based on sequence comparisons to public sequence databases using BLAST (National Center for Biotechnology Information) (Altschul et al. 1990). Each transcript was assigned a gene name through a comparison against UniProt protein sequence databases (SwissProt and TREMBL, version 2012_09) using BLASTx (e-value $<10^{-4}$ ). Gene names were based on best matches, excluding uninformative identifiers.

\subsubsection{Identification of Differentially Expressed (DE) genes}

All statistical tests were conducted using R (version 3.2.1) (Venables and Smith 2005). Statistical comparisons of RNA-Seq count data between the three symbiotic states were completed using the R package DESeq2 (version 1.9). DESeq2 was selected for differentially expression analysis because it utilises a variance stabilising procedure to transform count data into weighted expression values suitable for linear modelling. Raw $p$-values were adjusted for multiple test corrections and false discovery rate using the Benjamini-Hochberg procedure and a transcript was considered differentially expressed if the resulting adjusted $p$-value was $<0.1$, the default setting in DESeq2 (Love et al. 2014). Gene expression heatmaps were created with the pheatmap package in R (v3.1.3) (Kolde 2013).

\subsubsection{Functional summaries}

Annotated transcripts were assigned a gene ontology (GO) molecular function using BLAST2GO Pro (v.3.2.7) and manual search of Uniprot database (Consortium 2014), and grouped into putative functional categories based on literature and database searches (Hu et al. 2008; Kaniewska et al. 2012).

\subsubsection{Metabolite sample processing}

All sample-processing steps were performed at $4{ }^{\circ} \mathrm{C}$. Frozen anemones were thawed on ice, and pooled into single samples $(\mathrm{n}=30$ anemones per sample per treatment to ensure sufficient biomass for metabolite identification) and $500 \mu \mathrm{L}$ of cold $\left(4{ }^{\circ} \mathrm{C}\right)$ MilliQ water was added. Anemones were rapidly homogenised with a mechanical saw-tooth homogeniser (IKA T10 BS5, ThermoFisher Scientific, Auckland, New Zealand) for $1 \mathrm{~min}$ at a mid-speed setting. Once homogenised, $5 \mathrm{~mL}$ of cold $\left(4^{\circ} \mathrm{C}\right)$ MilliQ water was added, the sample thoroughly vortexed and a single $500 \mu \mathrm{L}$ aliquot removed for Symbiodinium cell density estimates (see Section 3.2.12). Symbionts were pelleted by centrifugation $\left(2,500 \times \mathrm{g}\right.$ for $5 \mathrm{~min}$ at $\left.4^{\circ} \mathrm{C}\right)$, and the host supernatant transferred and diluted with $5 \mathrm{~mL}$ of cold MilliQ water, followed by vigorous vortexing for $1 \mathrm{~min}$ and a second centrifugation $\left(2,500 \mathrm{xg}\right.$ for $5 \mathrm{~min}$ at $\left.4^{\circ} \mathrm{C}\right)$ to remove residual 
symbiont cells. Subsamples $(n=20)$ of the host material were confirmed for Symbiodinium absence by light microscopy (at x40 magnification). The cleaned host fractions were frozen at $-80{ }^{\circ} \mathrm{C}$ for 1 hour and lyophilised (FreeZone ${ }^{\circledR}$ Plus ${ }^{\mathrm{TM}} 4.5 \mathrm{~L}$ Cascade, Labconco, Auckland, New Zealand) for $18 \mathrm{~h}$ at $-105{ }^{\circ} \mathrm{C}$. To the symbiont pellets, $300 \mu \mathrm{L}$ of cold $\left(4{ }^{\circ} \mathrm{C}\right) \mathrm{MilliQ}$ water was added, the sample gently vortexed to resuspend the cells, and a single $500 \mu \mathrm{L}$ aliquot taken, to which $500 \mu \mathrm{L}$ of DNA buffer $(0.4 \mathrm{M} \mathrm{NaCl}, 0.05 \mathrm{M}$ EDTA) with $1 \%(\mathrm{w} / \mathrm{v})$ sodium dodecyl sulphate (SDS) was added, and the samples frozen at $-20^{\circ} \mathrm{C}$ for symbiont identification (see Section 3.2.13). The remaining symbiont material was frozen at $-80^{\circ} \mathrm{C}$.

\subsubsection{Symbiont density and protein quantification}

The aliquot of homogenate (Section 3.2.11) was centrifuged at $1,000 \times \mathrm{g}$ for $5 \mathrm{~min}$ to separate the animal and algal fractions. The host supernatant was collected, and the algal pellet resuspended in $1 \mathrm{~mL}$ FSW. Symbiodinium cell densities for metabolite profile normalisation were quantified using Improved Neubauer haemocytometer counts (Boeco, Germany) with a minimum of ten replicate counts per sample (i.e. to a confidence interval below 10\%). Cell density was normalised to soluble protein content of the host supernatant, which was assessed by the Bradford assay (Bradford 1976) and BSA as the standard. To test for differences in Symbiodinium cell densities between the treatments groups, assumptions of normality and homoscedasticity were assessed using Shapiro-Wilk and Levene's tests, respectively. As the data failed the assumptions of normality, and non-parametric Mann-Whitney $U$ rank-sum test was performed at the $p<0.05$ probability level using SPSS statistical software (v. 20, IBM Corp).

\subsubsection{Symbiont community identification from host samples}

For specimens used in RNA-seq, symbiont type was verified at the individual sample level (i.e. mRNA library-level) by direct sequencing of clade-specific reads at three loci known to be highly divergent between clades: internal transcribed spacer regions 1 and 2 of ribosomal DNA (ITS1, ITS2 rDNA) and chloroplast 23S rRNA (cp23S) (Ladner et al. 2012).

cDNA synthesis was performed using SuperScript ${ }^{\circledR}$ III First-strand synthesis system for RTPCR as per the manufacturer's instructions using a random hexamer primer (Invitrogen: Life technologies). The ITS1, ITS2 and cp23s regions were amplified using the outer primer

combinations S-Dino (5'-CGCTCCTACCGATTGAGTGA-3') and D1R-rev (5'ACCCGCTGAATTTAAGCATAT-3'), ITSintfor2 (5'-GAATTGCAGAACTCCGTG-3') and ITS2rev2 (5'-CCTCCGCTTACTTATATGCTT-3'), and cp23s 1 
(5'GCTGTAACTATAACGGTCC-3') and cp23s2 (5'-CCATCGTATTGAACCCAGC-3') respectively (Ladner et al. 2012; Hill et al. 2014). Samples were sequenced on an ABI 3730 capillary sequencing machine and their identities confirmed by reciprocal BLAST searches ( $n$ $=5)$.

For the metabolomics samples, direct sequencing of the ITS2 and cp23S regions was used to verify symbiont type. Symbiont pellets were preserved in 1\% SDS, and DNA was extracted using a CTAB-chloroform extraction protocol (dx.doi.org/10.17504/protocols.io.dyq7vv). Samples were immediately used for PCR, using a published thermal cycling regime and reaction mixture (Hill et al. 2014), and the ITS2 and cp23s primers mentioned above. PCR products were cleaned with ExoSAP-IT (USB Corporation, OH, USA) and sequenced by Macrogen (Seoul, South Korea). Sequences were aligned with Geneious v. 7.0 (Biomatters, Auckland, NZ) and a BLAST search was carried out against Symbiodinium ITS2 and cp23s sequences in GenBank.

\subsubsection{Intracellular metabolite extraction}

Extraction was based on the methods in Hillyer et al. (2016a). Once thoroughly dry, $1 \mathrm{~mL}$ of $100 \%$ methanol $\left(-20^{\circ} \mathrm{C}\right)$ containing the internal standard alanine-2,3,3,3- $\mathrm{d}_{4}$ (Sigma Aldrich, Auckland, New Zealand) was added to $30 \mathrm{mg}$ of lyophilised host material to extract the semipolar metabolites. The host fractions were sonicated for 30 mins at $4{ }^{\circ} \mathrm{C}$ in an ultrasonic bath (model: 8854 , Cole Parmer, USA) and centrifuged $\left(3,000 \times \mathrm{g}\right.$ for $30 \mathrm{~min}$ at $\left.4^{\circ} \mathrm{C}\right)$, and the supernatant containing the extracted metabolites was collected and stored at $-80^{\circ} \mathrm{C}$. Extraction was then repeated with $1 \mathrm{~mL}$ of $50 \% \mathrm{MeOH}\left(-20{ }^{\circ} \mathrm{C}\right)$ to extract the polar fraction. Samples were then centrifuged $\left(3,000 \times \mathrm{g}\right.$ for $30 \mathrm{~min}$ at $\left.4^{\circ} \mathrm{C}\right)$ and the supernatant pooled with the first extract. The total extract was then further centrifuged at $16,100 \times \mathrm{g}$ for $15 \mathrm{~min}$ at $4^{\circ} \mathrm{C}$ to ensure the removal of all particulates. Aliquots (total volume $200 \mu \mathrm{L}$ ) were then concentrated under vacuum (Eppendorf Concentrator 5301, Global Science, Auckland, New Zealand) at $30{ }^{\circ} \mathrm{C}$ until dry. The cell debris was frozen at $-20^{\circ} \mathrm{C}$ for protein quantification as determined by a modified Bradford colorimetric method (Smart et al. 2010).

Trimethylsilyl ester derivatisation of sugars, sugar alcohols and amino/non-amino acids for GC-MS was performed using an automated on-line derivatisation, adapted from established protocols (Roessner et al. 2000). All samples were re-dissolved in $20 \mu \mathrm{L}$ of $30 \mathrm{mg} \mathrm{mL}^{-1}$ methoxyamine hydrochloride in pyridine and derivatised at $37{ }^{\circ} \mathrm{C}$ for 120 min with mixing at $500 \mathrm{rpm}$. Twenty microlitres N,O-bis-(trimethylsilyl)trifluoroacetamide (BSTFA) and $1 \mu \mathrm{L}$ retention-time standard mixture comprised of $n$-alkanes were then added and the samples 
incubated for a further $30 \mathrm{~min}$ with mixing at $500 \mathrm{rpm}$. Each derivatised sample was then allowed to rest for 60 min prior to injection into the GC-MS. GC-MS analysis was performed on an Agilent 7890 gas chromatograph equipped with Gerstel MPS2 multipurpose sampler and coupled to an Agilent 5975C VL mass selective detector, run in splitless mode, with an injection volume of $1 \mu \mathrm{L}$ of each sample and a technical replicate of two injections per sample. Instrument control was performed with Agilent G1701A Revision E.02.01 ChemStation software. The gas chromatograph was fitted with a Varian Factor 4 column (VF-5ms; $30 \mathrm{~m} \times$ $0.25 \mathrm{~mm} \times 0.25 \mathrm{um}+10 \mathrm{~m}$ Ezi-guard). Helium (Ultra High Purity) was in constant flow mode at approximately $1 \mathrm{~mL} \mathrm{~min}^{-1}$ with retention-time locking applied. The oven temperature was started at $70^{\circ} \mathrm{C}$, held at this temperature for $1 \mathrm{~min}$ and then increased at $7^{\circ} \mathrm{C} \min ^{-1}$ to $325^{\circ} \mathrm{C}$, and finally held at this temperature for $3.5 \mathrm{~min}$.

The mass spectrometer quadrupole temperature was set at $150^{\circ} \mathrm{C}$, with the source set at $250^{\circ} \mathrm{C}$ and the transfer line held at $280^{\circ} \mathrm{C}$. Positive ion electron impact spectra at $70 \mathrm{eV}$ were recorded in scan mode with the following settings: a gain factor of 1.00 , detector threshold of 150 , mass to charge $(\mathrm{m} / \mathrm{z})$ range of $50-600 \mathrm{~m} / \mathrm{z}$ and solvent delay of $6.0 \mathrm{~min}$. Along with the samples, a series of known metabolite mixtures and solvent-only blanks were analysed. The metabolite mixtures contained the major compound classes being targeted, including amino acids (Amino Acid Standard solution AAS18, Sigma-Aldrich, Auckland), fatty acids (Menhaden fish oil, Sigma-Aldrich, Auckland), and sugars $(2.5 \mu \mathrm{M}$ ribitol, ribose, fructose, glucose, arabinose, galactose, mannitol, xylose, mannose and sucrose, Sigma-Aldrich, Auckland), and were analysed to confirm that these compounds could be accurately identified.

\subsubsection{Metabolite data extraction, pre-processing and normalisation}

Metabolite data extraction and analysis were performed with the use of AMDIS (http://chemdata.nist.gov/mass-spc/amdis) and Agilent MassHunter Workstation Software, Quantitative Analysis, vB.05.00/Build 5.0.291.0. Compound identification was based on an inhouse library of MS spectra, with retention indices based on n-alkane and the amino acid, fatty acid and sugar standards. Derivative peak areas were used to semi-quantify the concentrations of individual metabolites. Data was normalized to the final area of the standard alanine-2,3,3,3$\mathrm{d}_{4}$ and the protein content of the cell debris resulting from the samples' intracellular metabolite extraction using a modified Bradford colorimetric method (Smart et al. 2010). Data were then tested for normality and homogeneity, and data were $\log _{2}$ transformed if the assumption of normality was not met. Data were then autoscaled, and statistical analysis was performed using MetaboAnalyst 3.0 (http://www.metaboanalyst.ca) (Xia et al. 2015). Relative metabolite 
concentrations were evaluated by Principal Component Analysis (PCA). To specifically investigate how Symbiodinium genotype affects host metabolism, the fold change in relative metabolite abundance was calculated between B1-colonised hosts and D1a-colonised hosts before autoscaling and $\log _{2}$ normalisation. Metabolites were considered to be significantly differential when $t$ test $p$-value was $<0.05$ and the fold change (FC) was $>2$ for all five biological replicates. Visual exploration of the metabolites in the respective metabolic pathways was illustrated using Vanted (v2.6.2) (Junker et al. 2006; Rohn et al. 2012).

\subsubsection{Pathway analysis of symbiont type}

For integrated pathway analyses, a joint Wilcoxon pathway enrichment analysis of transcriptomics and metabolomics data was performed using the Integrated Molecular Pathway Level Analysis (IMPaLA) web interface (Kamburov et al. 2011). Input data consisted of the fold change in expression of the variance stabilised data of all Uniprot annotated genes $(13,535$ transcripts), and the $\log _{2}$ fold change in relative abundance between B1-colonised hosts and D1a-colonised hosts of the 85 metabolites possessing KEGG identifiers. Pathways were considered significantly affected if the FDR was $<0.05$ and pathways with less than three genes or metabolites measured were filtered out.

\subsection{Results}

\subsubsection{Symbiont type did not affect colonisation and autotrophic potential in Aiptasia}

To determine the ability of Aiptasia to form a successful and functional symbiosis with a novel Symbiodinium type, I compared the symbiont cell density and autotrophic potential (photosynthesis to respiration ratio) of anemones colonised with homologous type B1 and heterologous type D1a (Table 3.1). The two symbiont types reached the same densities in hosts (Mann Whitney $U, p=0.589, \mathrm{n}=12$ ). Furthermore, symbiont type had no effect on the rates of gross photosynthesis and symbiosis respiration (Students $t$-Test; $t=-2.173, \mathrm{df}=6, p=0.073$, and $t=-1.584, \mathrm{df}=3.55, p=0.197$, respectively), and hence the ratio of gross photosynthesis to respiration (P:R) was not different (Students $t$-Test; $t=-2.093, \mathrm{df}=6, p=0.081$ ), indicating a similar autotrophic potential in the two different symbioses. 
Table 3.1. Mean values for symbiont cell density and oxygen measurements of homologous type B1 and heterologous type D1a colonised Aiptasia. Values represent means \pm S.E.M $(n=4)$. Respiration (R) gross photosynthesis (Pgross) and the ratio are described.

\begin{tabular}{lll}
\hline Variable & Homologous type B1 & Heterologous type D1a \\
\hline $\begin{array}{l}\text { Symbiont cell density }\left(\text { cells } \mathrm{mg}^{-1}\right. \\
\text { protein) }\end{array}$ & $111324.02 \pm 28059.63$ & $105127.27 \pm 24704.47$ \\
$\mathrm{R}\left(\mu \mathrm{g} \mathrm{O} 2 \mathrm{mg}^{1}{ }^{1}\right.$ protein $\left.^{-1}\right)$ & $0.0054 \pm 0.0006$ & $0.0084 \pm 0.0018$ \\
Pgross $\left(\mu \mathrm{g} \mathrm{O} 2 \mathrm{mg}^{-1}\right.$ protein $\left.^{-1}\right)$ & $0.0074 \pm 0.0009$ & $0.0141 \pm 0.0029$ \\
Pgross:R & $0.6944 \pm 0.049$ & $0.8451 \pm 0.053$ \\
\hline
\end{tabular}

\subsubsection{Symbiotic state affects a range of molecular processes in the cnidarian host}

To characterise the underlying molecular response of a cnidarian host to the presence of different symbiont types, I compared the gene expression and metabolite profiles of aposymbiotic Aiptasia, those colonised with homologous B1, and those heterologous D1a symbionts.

\subsubsection{Transcriptomic analysis}

The final reference transcriptome was a concatenated transcriptome made up of both Aiptasia pallida and Symbiodinium clade B transcriptomes, and consisted of 58,160 individual transcripts after transcript isoforms were grouped and the longest representative chosen. After filtering for low counts per transcript, 35,517 individual transcripts remained and were tested for differential expression (Table S3.1). Principle component analysis (PCA) of the variancestabilised data (Appendix Table A1) revealed expression differences between the three symbiotic states (Figure 3.1A), with the greatest difference occurring between aposymbiotic and B1-colonised anemones, with D1a-colonised hosts showing an overlap between the two other symbiotic states. The PCA shows that host gene expression in a novel association is intermediate between having no symbiont and hosting the homologous type. Statistical analysis of the concatenated variance-stabilised data in DESeq2 identified 720 differentially expressed (DE) genes according to symbiotic status (Appendix Table A2). Separation of Symbiodinium transcripts from the Aiptasia genes ensured that all conclusions were drawn based only on host gene expression. After the Symbiodinium DE genes were removed, 686 DE genes remained (Appendix Table A3). PCA of the DE genes shows a distinctive separation between the symbiotic states (Figure $3.1 \mathrm{~B}$ ). PC axis 1 accounts for $47.7 \%$ of the explained variation, and 
the spread of the data in the PCA indicates that gene expression of anemones with heterologous symbionts was more similar to aposymbiotic anemones than to hosts with homologous symbionts. The expression heatmap of the DE genes within the three symbiotic states similarly shows closer alignment between D1a-colonised and aposymbiotic anemones, with B1colonised anemones being the most different. (Figure 3.1C).
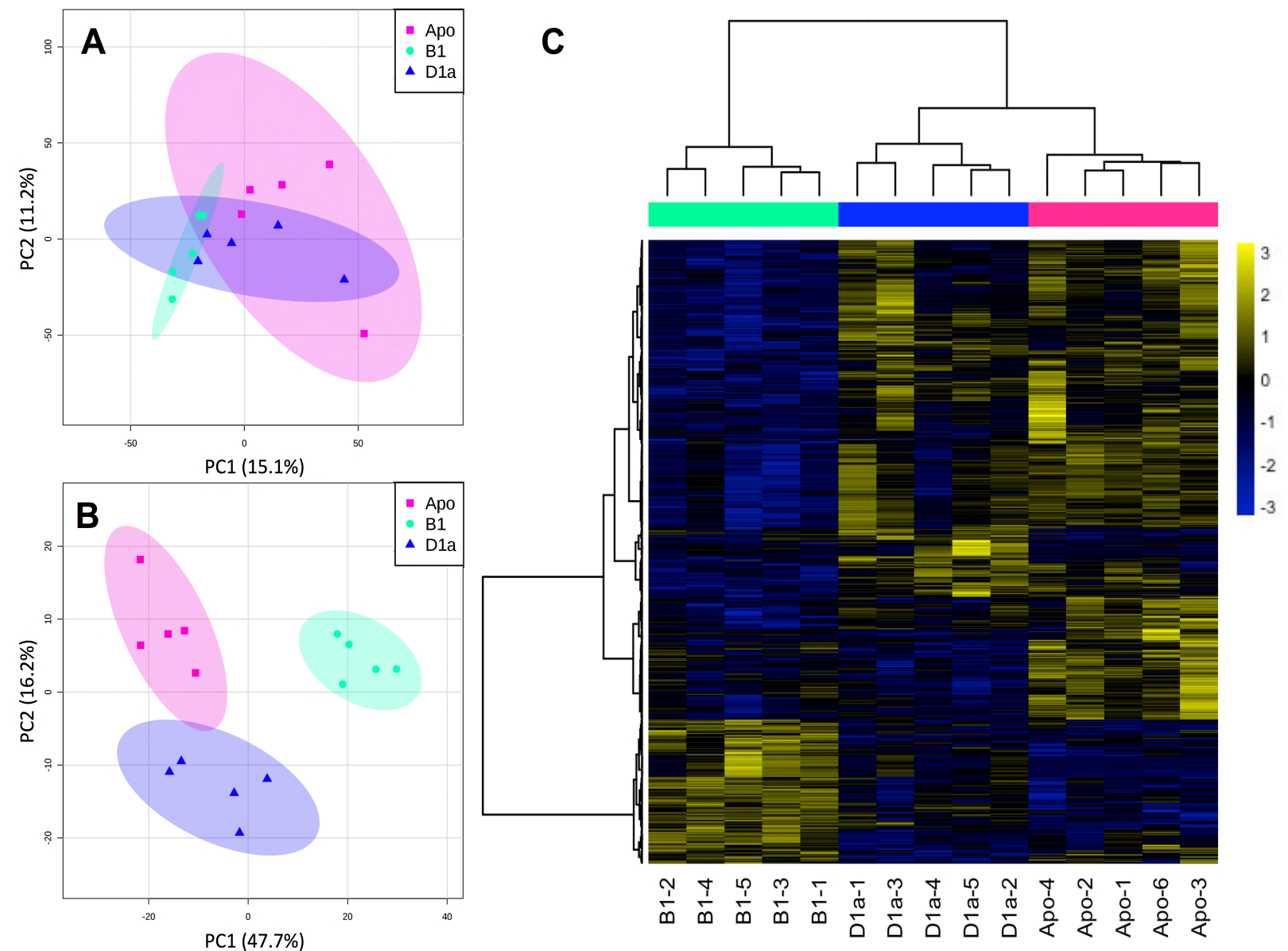

Figure 3.1 Aiptasia sp. host gene expression in response to symbiotic state. (A) Principle component analysis (PCA) of all genes. (B) PCA of all differentially expressed genes (DEGs) clusters replicate samples by symbiotic state (FDR 10\%) (C) Heat map of all DEGs $(p<0.1)$. Rows are genes, columns are samples as ordered by hierarchical clustering of gene expression pattern; homologous (B1), heterologous (D1a) and aposymbiotic (Apo). The colour scale is $\log _{2}$ (fold change relative to the genes mean). The tree is a hierarchical clustering of genes based on Pearson's correlation of their expression across samples.

\subsubsection{Annotation of differentially expressed transcripts}

Of the $686 \mathrm{DE}$ genes, 409 had protein annotations (UniProt) by homology to known genes (BLASTx; E value cut off of $1 \times 10^{-5}$ ) (Appendix Table A3). My subsequent analyses focused on annotated transcripts. 
The annotated DE genes were assigned a gene ontology (GO) molecular function, and subsequently allocated to a broad functional category: cell cycle, metabolism, stress response, transport, cytoskeleton, extracellular matrix, catalytic/enzymatic activity, and binding (Table 3.2). Forty-five genes were not assignable to a $\mathrm{GO}$ molecular function (i.e. uncharacterised proteins), and were excluded from further analyses. Nineteen percent of the annotated DE genes fell under cell cycle, the majority of which were involved in DNA regulation processes, such as transcription factors. Metabolism made up $17 \%$ of the DE genes, in which amino acid and protein metabolism featured prominently. Carbohydrate metabolism included glycosylphosphatidylinositol-linked carbonic anhydrase, implicated in inorganic carbon transport, and a Niemann-pick type C2 fragment. Additionally, multiple DE genes grouped in the synthesis and degradation of lipids category. Stress response made up $16 \%$ of the DE genes, comprised primarily of genes involved in redox homeostasis, including glutathione-related enzymes, heatshock proteins, and three cytochrome p450 genes. Secondly, a large number were involved in apoptosis, such as caspase- 8 . Transport-related proteins made up 13\% of the DE genes, and included a large number involved in metabolite transport, including $\mathrm{ABC}$ transporter proteins, solute-carrier (SLC) proteins, monocarboxylate transporters, and phosphatidylinositol signalling proteins. Proteins involved in oxygen transport and G-protein receptor signal transduction were also members of this segment. 
Table 3.2. Summary of the main molecular processes differentially expressed across the three symbiotic states (homologous, heterologous and aposymbiotic host anemones). Differentially expressed (DE) genes were assigned a Gene Ontology molecular function and grouped into putative categories based on GO slimming and literature searches. $\log _{2}$ fold change is relative to the genes mean. Font colour indicates upregulated (red) and downregulated (blue) expression.

\begin{tabular}{|c|c|c|c|}
\hline DE transcripts & $\mathbf{N}$ & Uniprot & Molecular Process \\
\hline Binding & 23 & & \\
\hline Calmodulin binding & & F1AON9 & Calcium ion binding \\
\hline CnidEF ( $n=2)$ & & Q2ESH8 & Calcium ion binding \\
\hline Catalytic/Enzymatic activity & 48 & & \\
\hline TauD & & B7JWG7 & Sulphur transferase enzyme \\
\hline Cell Cycle & 70 & & \\
\hline F3 ubiauitin-nrotein ligagse & & O4KLN8 & Protein modification \\
\hline Molecular chaperone & & B7Q4C2 & Protein modification \\
\hline Ubiquitin domain-containing protein & & Q54MD2 & Protein modification \\
\hline Cytoskeleton & 35 & & \\
\hline Actin binding protein & & Q8H1L9 & Actin binding \\
\hline Actin binding protein & & Q6WE55 & Actin binding \\
\hline Actin binding protein & & P28575 & Actin binding \\
\hline i Beta gamma crystallin isoform 8 & & A8C9L8 & Cytoskeletal protein binding \\
\hline Echinonectin & & 076470 & Cytoskeletal protein binding \\
\hline Nectin & & Q70JAO & Cytoskeletal protein binding \\
\hline Extracellular matrix & 21 & & \\
\hline ; Collagen trimer alpha-1(III) & & Q08E14 & Collagen binding \\
\hline Collagen trimer alpha- $1(\mathrm{XI})$ & & Q61245 & Collagen binding \\
\hline ( Collagen trimer alpha-1)!) & & Q9YIB4 & Collagen binding \\
\hline ; Collagen trimer alpha- $5(\mathrm{VI})(\mathrm{n}=2)$ & & G3GZJO & Collagen binding \\
\hline Metabolism & 62 & & \\
\hline Arginine kinase activity & & 015992 & Amino acid metabolism \\
\hline Ornithine decarboxylase antizyme & & B2CR15 & Amino acid metabolism \\
\hline Phenylalanine tRNA synthetase & & Q5EBG3 & Amino acid metabolism \\
\hline I Proline catabolism & & Q4V7V6 & Amino acid metabolism \\
\hline Serine/threonine protein kinase & & F2ULY7 & Amino acid metabolism \\
\hline Carbohydrate metabolism & & A3HSO7 & Carbohydrate metabolism \\
\hline Carbohydrate metabolism & & I3IDI2 & Carbohydrate metabolism \\
\hline Glycophosphatidylinositol-linked carbonic anhydrase & & A3FFY2 & Carbohydrate metabolism \\
\hline i Polysaccharide lyase activity & & FOS761 & Carbohydrate metabolism \\
\hline Long-chain fatty acyl-CoA synthetase 1 (ACSL 1) & & E2D780 & Fatty acid metabolism \\
\hline Cytosolic phospholipase A2 & & A4IFJ5 & Arachidonic acid metabolism \\
\hline Phospholipase A2 & & A7LCJ2 & Arachidonic acid metabolism \\
\hline Cryptochrome 2 & & A212PO & Gluconeogenesis \\
\hline Lipase & & A7SL62 & Lipid metabolism \\
\hline Lipoxygenase & & F2Z929 & Lipid metabolism \\
\hline Phosphatidylinositol-Specific Phospholipase & & D4A4P6 & Inositol-phosphate metabolism \\
\hline : Triglyceride lipase & & BЗVMK3 & Lipid metabolism \\
\hline Carboxypeptidase A2 (n=2) & & P48052 & Protein metabolism \\
\hline Carboxypeptidase B & & E6ZIX2 & Protein metabolism \\
\hline Larboxypeptidase D & & EOVR63 & Protein metabolism \\
\hline S-adenosylmethionine synthase & & A7RTZ9 & S-Adenosylmethionine synthase activity \\
\hline S-adenosylmethionine synthase & & A7SEP5 & S-Adenosylmethionine synthase activity \\
\hline Niemann-Pick disease C2 & & Q53HV6 & Sterol metabolism \\
\hline Arginine translocation pathway signal & & Q476C5 & Urea cycle \\
\hline Glutamate dehydrogenase & & A7SE06 & Urea cycle \\
\hline Stress response & 58 & & \\
\hline Apoptosis & & Q5PQ83 & Apoptosis \\
\hline : Apoptosis & & B4DGI3 & Apoptosis \\
\hline Caspase 8 & & Q2LGB8 & Apoptosis \\
\hline Fibroblast growth factor receptor & & Q60818 & Apoptosis \\
\hline Oxidative stress induced growth inhibitor & & Q2KIN7 & Apoptosis \\
\hline TNF receptor 2 & & A2TK67 & Apoptosis \\
\hline TNF receptor 3 & & A2TK68 & Apoptosis \\
\hline : TNF receptor 6 & & A2TK70 & Apoptosis \\
\hline Tumor suppression gene & & Q6VQ14 & Apoptosis \\
\hline Cytochrome P450 & & A7TVD1 & Cytochrome activity \\
\hline Cytochrome P450 & & 042457 & Cytochrome activity \\
\hline ; Cytochrome P450 & & G7K3L7 & Cytochrome activity \\
\hline ER stress associated protein & & F1QWT4 & ER Stress response \\
\hline ER stress associated protein & & QOV9K8 & ER Stress response \\
\hline Glutamate-cysteine ligase catalytic subtunit & & B2ZG39 & Glutathione synthase activity \\
\hline GSS & & Q5XJT8 & Glutathione synthase activity \\
\hline Glutathione peptidase & & Q4PMFO & Glutathione synthase activity \\
\hline Cytosolic glutathione S-transferase-2 & & B2Z137 & Glutathione transferase activity \\
\hline Glutathione-S-transferase & & CONY9O & Glutathione transferase activity \\
\hline DNAJ C3 & & Q7ZWH5 & Heat shock \\
\hline DNAJ C3 & & Q9ZWK3 & Heat shock \\
\hline Macrophage expressed protein & & F8RU73 & Immune response \\
\hline Oxidoreductase & & A7RUM6 & Oxidoreductase activity \\
\hline Redox enzyme & & Q2KHP7 & Oxidoreductase activity \\
\hline Sulfiredoxin-1 & & EOVWL8 & Oxidoreductase activity \\
\hline Xanthine dehydrogenase & & Q6NSO3 & Oxidoreductase activity \\
\hline Xanthine dehydrogenase & & F4WWU5 & Oxidoreductase activity \\
\hline 3 Coenzyme Q6 & & Q6DF46 & Oxidoreductase activity \\
\hline Protein disulphide isomerase & & Q7PM55 & Redox homeostasis \\
\hline Thioredoxin domain containing protein & & Q7TN22 & Redox homeostasis \\
\hline Transport & 48 & & \\
\hline ; ABCD4 & & 014678 & $A B C$ transporter activity \\
\hline Monocarboxylate membrane transporter & & EOVEK5 & Amino acid transport \\
\hline Monocarboxylate membrane transporter (SLC16A10) & & A1L1W9 & Amino acid transport \\
\hline Monocarboxylate membrane transporter (SLC16A10) & & Q3U9N9 & Amino acid transport \\
\hline G-protein coupled receptor & & B2RXV6 & Cellular signalling \\
\hline G-protein singalling protein & & A5PLK6 & Cellular signalling \\
\hline Lipid transport activity & & F4WT31 & Lipid transport \\
\hline Non-specific lipid-transfer protein & & 062742 & Lipid transport \\
\hline Phosphatidylinositol signalling & & F2Z2U4 & Phosphatidylinositol signalling \\
\hline : Phosphatidylinositol signalling & & E9CCM6 & Phosphatidylinositol signalling \\
\hline Sodium phosphate cotransporter (SLC34A2) & & Q9PT83 & SLC transporter activity \\
\hline Transmembrane transport protein (SLC25A22) & & Q6P4S3 & SLC transporter activity \\
\hline Zinc transporter (SLC39A10) & & Q9ULF5 & SLC transporter activity \\
\hline Macrophage mannose receptor & & Q2HZ94 & Sugar transport \\
\hline Sugar phosphate exchanger 2 (SLC37A2) & & Q9WU81 & Sugar transport \\
\hline
\end{tabular}




\subsubsection{Metabolite profile comparison of the three symbiotic states}

A total of 85 compounds were identified in the polar and semi-polar extracts of free metabolite pools from hosts, consisting primarily of amino acids, intermediates, organic acids and amides, sugars and fatty acids (Table S3.2). PCA analysis (Figure 3.2) shows distinct clustering of the three symbiotic states. Similar to the PCA analysis of expression profiles the greatest difference occurred between aposymbiotic and homologous B1-colonised anemones along PC axis 1 (46.7\% of the explained variation), while the heterologous D1a-colonised hosts span the intermediate region.

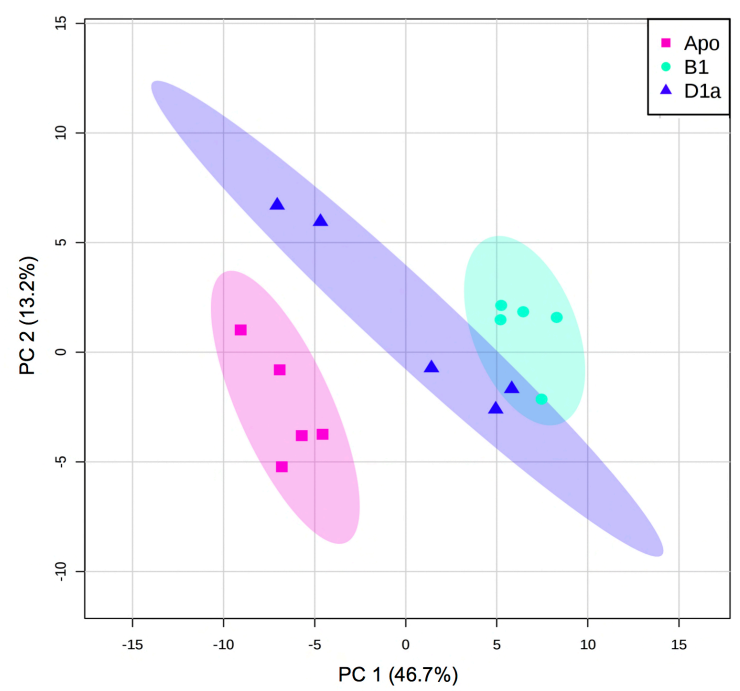

Figure 3.2 Host metabolite profile PCA scores plot, with $95 \%$ confidence intervals. The relative abundance of the compounds detected in host free pools between the three symbiotic states (with $95 \%$ confidence regions) $(n=5)$.

\subsubsection{Symbiont type affects host metabolite pools}

Of the 85 metabolites identified, eight were of higher relative abundance in B1-colonised host pools (Figure 3.3, Table S3.3). The remaining 77 metabolites were of higher abundance in the D1a-colonised host tissues, including all nine unsaturated fatty acids detected. Univariate statistical analysis of the fold-change in relative abundance of each compound between B1colonised and D1a-colonised host pools identified ten compounds that were significantly higher in abundance in D1a-colonised host pools, while no compounds were significantly lower in D1a-colonised host pools (Figure 3.3, Table S3.3). Two mono-unsaturated fatty acids (MUFAs) - palmitoleic acid (C16:1) and oleic acid (C18:1) - and three polyunsaturated fatty acids (PUFAs) - linoleic acid (C18:2), linolenic acid (C18:3) and dihomo- $\gamma$-linolenic acid (C20:3) - were significantly higher in D1a-colonised host tissues. Saturated fatty acid butyric acid (C4:0), amino acids methionine and tyrosine, the amino acid-derivative tyramine, CoA precursor pantothenic acid, and amino-sugar glucosamine were present at a significantly higher in abundance in D1a-colonised hosts (Figure 3.3, Table S3.3). 


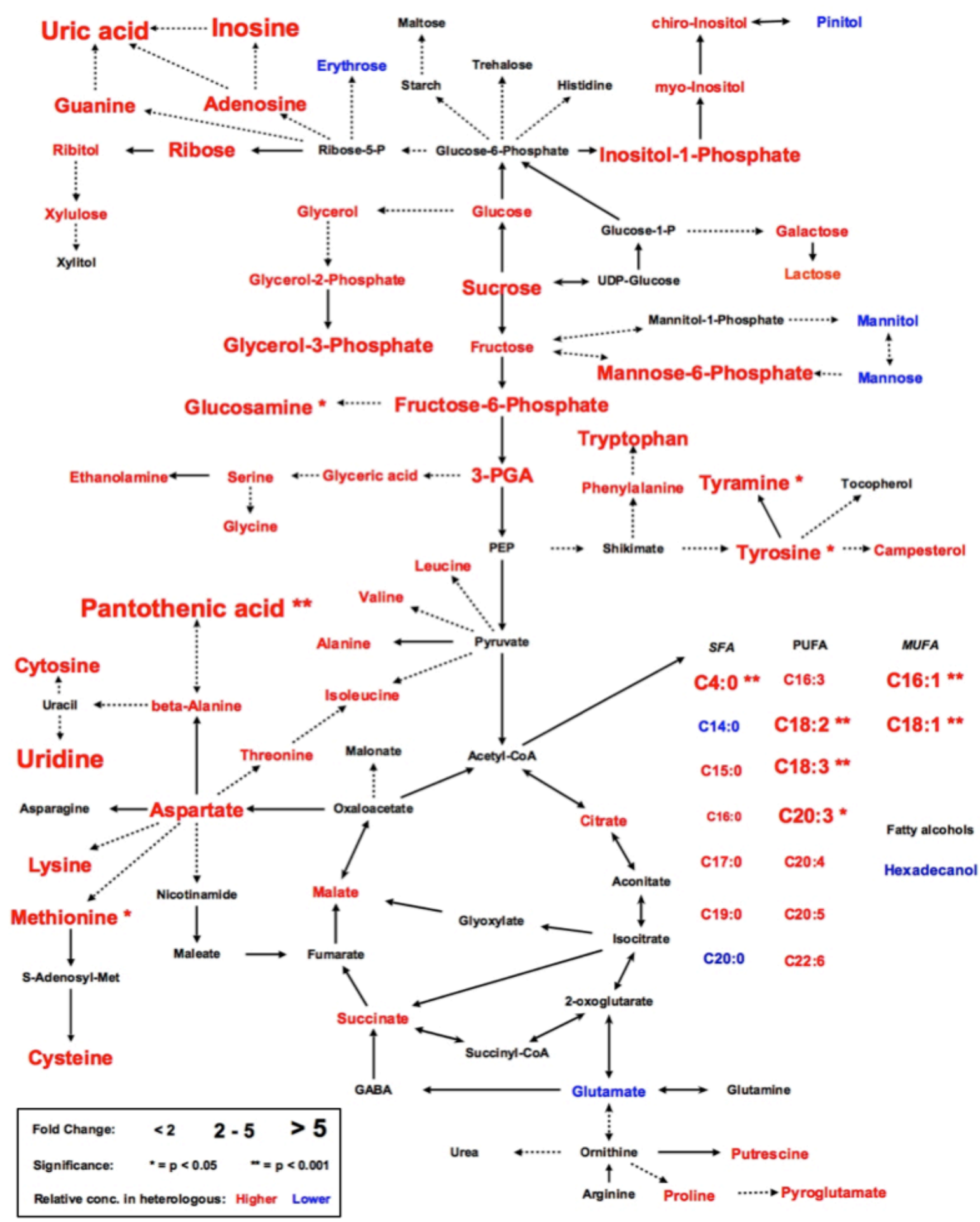

Figure 3.3 Metabolic pathway visualisation of the fold-change in relative abundance of compounds detected in host free pools between heterologous and homologous associations. Font size represents fold change of relative abundance in heterologous host tissues. Asterisks denote compounds that differed significantly $(p<0.05)$ between symbiont types. Font colour shows whether compound abundance was higher (red) or lower (blue) in abundance in heterologous host tissues, or represent intermediates not detected in the metabolite data (black). 


\subsubsection{Symbiosis with a heterologous symbiont type induces increased catabolism of stored energy, metabolite signalling pathways and cellular redox responses}

Joint enrichment analysis of the transcriptomic and metabolomics data revealed that 22 pathways were affected when Aiptasia was in symbiosis with heterologous symbiont type D1a (Appendix Table A4). Five of the reported pathways referred to broad categories as defined in the Reactome database ('metabolism', 'transport of small molecules', diseases', 'signal transduction' and 'gene expression') (Milacic et al. 2012; Croft et al. 2014), and 17 refer to specific pathways within these categories (Table 3.3).

Because the described molecular pathway databases are strongly biased towards well-described processes in model organisms, the integrated pathway results are limited in their usefulness in interpreting the results in regards to cnidarian-Symbiodinium interactions (Mohamed et al. 2016). Consequently, the joint pathway enrichment analysis results and the expression patterns of the individual entities (genes metabolites) were used to further group the pathways into the following categories based on literature searches highlighting gene and metabolite functions likely involved in the coral-Symbiodinium symbiosis: sugar storage and transport; lipid and protein metabolism and transport; cellular oxidative homeostasis, and molecular signalling pathways (Table 3.3; Figure 3.4).

\subsubsection{Sugar transport and storage}

Pathways involved in glycogen storage disorders were identified in the integrated pathway results, indicating a reduction in glycogen storage in D1a-colonised host tissues (Figure 3.4A, Table 3.3). Ten genes and ten metabolites specifically involved in glycogen storage disorders were detected, including the glucan branching enzyme GBE1, which is involved in glycogen synthesis and was downregulated in D1a-colonised hosts. I also observed a downregulation of multiple sugar transporters, including the sodium/glucose co-transporter SGLT4 (SLC5A9) (Baumgarten et al. 2015), and a putative sodium-dependent glucose transporter (KIAA1919), as well as multiple sugar-inositol transporters. The proton myo-inositol cotransporter HMIT (SLC2A13) is a member of the facilitative glucose transporter family that has been recently identified in the Aiptasia genome (Baumgarten et al. 2015), and was downregulated in the D1a-colonised host. Also downregulated were the $m y o$-inositol $/ \mathrm{Na}^{+}$co-transporter SMIT1 (SLC5A3), involved in sugar transport and signalling (Wright and Turk 2004), and the myo/chiro-inositol/glucose transporter SMIT2 (SLC5A11), previously found to be highly upregulated in symbiotic anemones (Lehnert et al. 2014; Hemond and Vollmer 2015). The decreased activity of these transporters will result in a reduction in the inter- and/or 
intracellular movement of sugars, yet I observed a high abundance of free pools of carbohydrates in the Dla-colonised host tissues. Concertedly, this indicates defects in the processing of glycogen synthesis and an increase in stored-sugar catabolism, in the D1acolonised anemones.

Table 3.3. A summary of the significantly enriched pathways $(F D R<0.05$ and at least 3 genes or metabolites present) per IMPaLa analysis of heterologous symbiont-containing hosts against homologous symbiont-containing hosts. Font colour indicates number of downregulated (blue) and upregulated (red) expression in the heterologous host tissues. An identification list of the genes and metabolites in each pathway can be found in Appendix Table A4. Capital letter in superscript denotes the putative biological response the pathway is included in: Sugar storage, transport and signalling (A); Lipid and protein metabolism and transport (B); Oxidative stress response (C); Molecular signalling pathway (D).

\begin{tabular}{|c|c|c|c|}
\hline Pathway name & $p$ value & $\begin{array}{l}\text { Measured } \\
\text { genes }\end{array}$ & $\begin{array}{l}\text { Measured } \\
\text { metabolites }\end{array}$ \\
\hline Metabolism & $<0.0001$ & & \\
\hline Biological oxidations ${ }^{\mathrm{C}}$ & $<0.001$ & $1 / 2$ & $1 / 22$ \\
\hline $\begin{array}{l}\text { Metabolism of amino acids and derivatives (including Urea } \\
\text { cycle and metabolism of arginine, proline, glutamate, } \\
\text { aspartate, and asparagine) } \mathrm{B}, \mathrm{C}\end{array}$ & $<0.001$ & $2 / 7$ & $1 / 24$ \\
\hline Tyrosine metabolism $\mathrm{B}, \mathrm{C}$ & 0.026 & $0 / 3$ & $1 / 7$ \\
\hline Metabolism of vitamins and cofactors ${ }^{C}$ & 0.036 & $2 / 1$ & $1 / 8$ \\
\hline Metabolism of lipids and lipoproteins ${ }^{B}$ & 0.007 & $7 / 5$ & $2 / 16$ \\
\hline Protein digestion and absorption ${ }^{B}$ & $<0.001$ & $2 / 2$ & $1 / 19$ \\
\hline Fatty acid triacylglycerol and ketone body metabolism $\mathrm{B}, \mathrm{D}$ & $<0.001$ & $3 / 2$ & $0 / 10$ \\
\hline Purine metabolism $\mathrm{C}$ & 0.014 & $3 / 1$ & $1 / 8$ \\
\hline Transmembrane transport of small molecules & $<0.001$ & & \\
\hline SLC-mediated transmembrane transport $\mathrm{A}, \mathrm{B}$ & $<0.001$ & $5 / 4$ & $2 / 38$ \\
\hline Transport of glucose and other sugars ${ }^{A}$ & $<0.001$ & $3 / 1$ & $0 / 22$ \\
\hline$A B C$ transporters ${ }^{B}$ & $<0.001$ & $3 / 1$ & $1 / 25$ \\
\hline Diseases & $<0.001$ & & \\
\hline Glycogen storage diseases ${ }^{A}$ & 0.039 & $6 / 7$ & $1 / 9$ \\
\hline Metabolic disorders of biological oxidation enzymes ${ }^{C}$ & $<0.001$ & $3 / 6$ & $1 / 33$ \\
\hline Signal Transduction & $<0.001$ & & \\
\hline Signaling by GPCRD & 0.001 & $6 / 4$ & $1 / 16$ \\
\hline GPCR ligand binding ${ }^{D}$ & 0.006 & $3 / 3$ & $1 / 13$ \\
\hline Class A/1 (Rhodopsin-like receptors) ${ }^{\mathrm{D}}$ & 0.008 & $1 / 2$ & $0 / 11$ \\
\hline GPCR downstream signaling ${ }^{\mathrm{D}}$ & 0.022 & $5 / 3$ & $1 / 9$ \\
\hline Gene Expression $^{A, B, C, D}$ & $<0.001$ & $6 / 9$ & $1 / 17$ \\
\hline
\end{tabular}




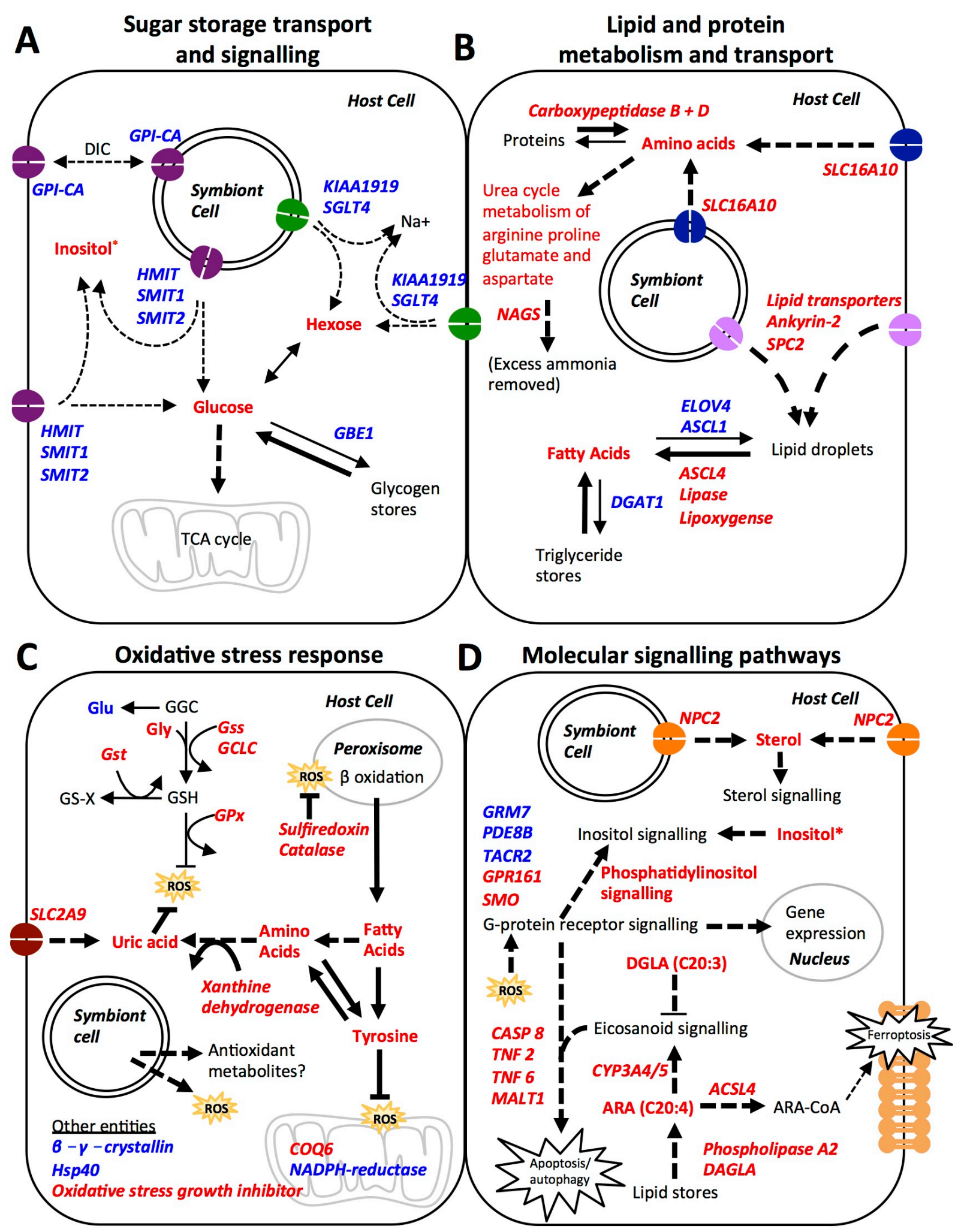

*Multiple inositol isoforms and inositol phosphates were detected, but are collectively referred to here as 'Inositol'

Figure 3.4 General overview of significantly affected pathways as indicated by differentially expressed genes and metabolites in the heterologous host tissues. Font colour indicates upregulated (red) and downregulated (blue) expression in the heterologous host tissues. Black font entities were not measured in this study. Arrow thickness indicates direction of pathway activity, and dashed line indicates putative activity. Italics represent genes, and non-italicised represents metabolite; abbreviations are as described in the main text. Letters refer to descriptions in the main text. 


\subsubsection{2. $\quad$ Lipid and protein metabolism and transport}

Pathways involved in lipid and fatty acid degradation and transport were upregulated in D1acolonised hosts, and enzymes responsible for fatty acid synthesis and elongation were downregulated (Figure 3.4B, Table 3.3). In addition I observed a higher abundance in five saturated and six unsaturated free fatty acids in D1a-colonised host metabolite pools, likely a result of lipid degradation (Figure 3.4B, Table 3.3). Evidence for this was observed in the metabolism of triglycerides; triglycerides are known to be a major storage form of lipids in corals (Harland et al. 1993), and two triglyceride lipases were upregulated in D1a-colonised hosts, while an enzyme involved in the formation of triglycerides (DGAT1) was downregulated. Concurrently, two genes with lipid transport molecular functions (Ankyrin-2 and SPC2) were upregulated in the D1a-colonised hosts. Meanwhile, an enzyme involved in lipid biosynthesis (ACSL1; a member of the acyl-CoA synthetase long-chain family), and the very-long chain fatty acid elongase (ELOVL4), were upregulated in B1-colonised hosts. Simultaneously, I detected a lower relative abundance of many fatty acids in B1-colonised host tissues. Together, this indicates fatty acids were converted into lipids for storage in the B1colonised hosts.

Symbiosis with the heterologous symbiont type D1a resulted in a number of changes in protein and amino acid synthesis, transport and metabolism in the cnidarian host (Figure 3.4B, Table 3.3). The relative abundance of all detected amino acids was higher in Dla-colonised tissues. Multiple enzymes involved in the catabolism of proteins and amino acids, and three monocarboxylate transporters, involved in the translocation of aromatic amino acids, were upregulated in D1a-colonised hosts (two encoding SLC16A10 and one putative member). Specifically, pathway analysis revealed an upregulation of the urea cycle and metabolism of arginine, proline, glutamate and aspartate, and tyrosine metabolism (Table 3.3). An upregulation of $\mathrm{N}$-acetylglutamate synthase (NAGS), which helps to remove excess ammonia, was observed in the D1a-colonised hosts. Although urea and arginine were not detected in the metabolite profiles, aspartate, and proline were more abundant in the D1a-colonised host, while glutamate was more abundant in the B1-colonised host.

\subsubsection{Oxidative stress response}

The D1a-colonised hosts showed a significant activation of processes involved in cellular oxidative stress responses, including reactive oxygen species- (ROS) and reactive nitrogen species- (RNS) producing pathways, oxidative stress response proteins, and the synthesis of enzymatic and non-enzymatic antioxidants (Figure 3.4C, Table 3.3). 
Stress response protein Hsp40 and beta gamma crystalline proteins may assist in preventing damage from oxidative stress and ultimately the induction of host cell apoptosis (Weis 2008), and were upregulated in the B1-colonised association, indicating pathways are potentially more active in preventing cellular oxidative damage in a homologous association, than in a novel association.

Mitochondrial respiration produces ROS from oxidation of NADPH, catalysed by NADPH oxidases, while NADPH reductases (e.g. CYP450) contribute to the removal of ROS (Murphy 2009). A number of NADPH reductases were downregulated, perhaps resulting in ROS accumulation in the heterologous host tissues. Coenzyme Q6, which is produced in response to mitochondrial hypoxia, was found to be upregulated in Dla-colonised hosts. The enzyme sulfiredoxin, which is involved in antioxidant metabolism by reactivating peroxiredoxins, a group of peroxidases, and a catalase enzyme, were upregulated in D1a-colonised hosts.

Xanthine dehydrogenase, primarily involved in purine metabolism and the formation of uric acid, was upregulated in D1a-colonised hosts. Similarly, uric acid was significantly more abundant in D1a-colonised hosts. Uric acid is a strong non-enzymatic antioxidant and reductant, scavenging both $\mathrm{O}^{\circ}$ and $\mathrm{H}_{2} \mathrm{O}_{2}$. A transporter of uric acid (SLC2A9) was also found to be upregulated in D1a-colonised hosts. Mycosporine-like amino acid's (MAAs), such as tyrosine, have functional roles as ROS scavengers. They are produced via the shikimate pathway, which has been identified in both cnidarian hosts and the algal partner, and ROS stress has been shown to increase biosynthesis of these compounds (Rosic and Dove 2011). Tyrosine metabolism was identified as significantly different, and tyrosine and its derivative tyramine were significantly more abundant in the D1a-colonised host.

Glutathione (GSH) can detoxify ROS directly or as an electron donor in a reaction catalysed by glutathione peroxidase. Glutathione peroxidase was upregulated in D1a-colonised host tissues, as was glutathione synthase (Gss) and glutamate-cysteine ligase (GCLC), both enzymes involved in the synthesis of glutathione. Glutathione-S-transferase (Gst) is responsible for catalysing the conjugation of reduced GSH for the purpose of detoxification; two were upregulated in Dla-colonised hosts, including one identified as cystolic. Glutamate is a glutathione precursor and pool abundance was lower in D1a-colonised hosts, corresponding with an upregulation in GSH synthesis.

\subsubsection{Molecular signalling pathways}

The results show an upregulation of $\mathrm{G}$ protein-associated stress signalling and eicosanoid signalling pathways in the heterologous hosts (Figure 3.4D, Table 3.3). 
G-protein coupled receptors (GPCRs) are involved phosphatidylinositol signalling and initiation of cellular responses such as apoptosis. There was an upregulation of multiple entities involved in apoptotic processes, such as TNF receptor associated factors 2 and 3, MALT1 paracaspase (which has previously been found in cnidarians and which activates transcription of NF-kB) (Hulpiau et al. 2016), and caspase-8 (an autophagic cell death enzyme (Dunn et al. 2007; Hawkins et al. 2013)). Furthermore, multiple inositol isoforms and inositol-phosphates were at higher abundances in the D1a-colonised host tissues (Table S3.6).

Diacylglycerol lipase (DAGLA) and phospholipase A2 were upregulated in D1a-colonised hosts. These both hydrolyse lipids to cause a disproportionate release of arachidonic acid (ARA; C20:4). ARA is an essential PUFA and is a precursor to the highly biologically active eicosanoids, and has been suggested to play a key role in coral immunity, initiating the caspase-3 apoptosis pathway (Savchenko et al. 2010; Libro et al. 2013; Lõhelaid et al. 2015). A higher abundance of ARA was found in heterologous host tissues, along with two cytochrome P450 mono-oxygenase enzymes (CYP3A4/5) which catalyse ARA to signalling derivatives (and which involve the reduction of $\mathrm{O}_{2}$ and the production of $\mathrm{H}_{2} \mathrm{O}_{2}$ (Lesser 2006)). Simultaneously upregulated was acyl-CoA synthetase long-chain family member 4 (ACSL4), which is distinguished by its preference for metabolising ARA into arachidonoyl-CoA. If arachidonoyl-CoA is subsequently inserted into lipid membranes and oxidised due to cellular oxidative stress, ferroptosis cell death is induced (Magtanong et al. 2016). In this regard, I observed a significantly higher abundance of dihomo- $\gamma$-linolenic acid (DGLA) (C20:3) in D1acolonised hosts, which acts as an antagonist of the ARA leukotriene signalling cascade, serving to counter the inflammatory effects of ARA. DGLA is a product of elongation of C18:3n-6, which in turn is a product of desaturation of $\mathrm{C} 18: 2 \mathrm{n}-6$, the latter of which has been previously suggested to be a symbiont-only ability (Dunn et al. 2012); both of which were significantly more abundant in D1a-colonised host tissues (Figure 3.4D, Table S3.3).

\subsection{Discussion}

A coral's response to environmental fluctuations is heavily influenced by the physiology of the resident symbiont population. Changes in symbiont composition may therefore provide a mechanism for environmental acclimatisation and adaptation in corals. However, specificity in the symbiosis is likely to restrict the ability to form novel associations. Nevertheless, the underlying mechanisms of symbiosis specificity remain unclear. This study is the first to combine transcriptomic and metabolomic data analysis to examine symbiosis specificity 
between cnidarians and symbiotic dinoflagellates. I have shown that when the cnidarian host Aiptasia forms a symbiosis with a novel symbiont type, its gene and metabolite expression patterns are intermediate between the aposymbiotic and 'normal' symbiotic states. The presence of a novel symbiont type in Aiptasia induced the catabolism of host energy stores, disrupted cellular oxidative homeostasis, and resulted in complex metabolic signalling cascades. Collectively, these processes may ultimately result in the breakdown of the symbiosis or, alternatively, allow for the adaptive tolerance of novel symbiont types. These effects could not be attributed to differences in the symbiont cell density, or different rates of photosynthesis or respiration, as these were the same irrespective of symbiont type.

\subsubsection{Heterologous symbiosis affects gene and metabolite expression profiles}

The results demonstrated that Aiptasia was able to form a functional symbiosis with the heterologous Symbiodinium type D1a, but that the expression of genes and metabolites was intermediate between hosting the 'normal' symbiont, and having no symbiont. The ability of Aiptasia to form an association with a heterologous symbiont is consistent with previous studies (Schoenberg and Trench 1980b; Starzak et al. 2014; Matthews et al. 2016). Nevertheless, it is still unclear whether the symbiont goes unnoticed by the host upon colonisation or whether the symbiont is able to manipulate the host's response accordingly, either through modification or suppression of host gene expression, or both (Voolstra et al. 2009; Schnitzler and Weis 2010; Farnese et al. 2016; Mohamed et al. 2016). Interestingly, my study identified differential expression of multiple host cell-cycle proteins in response to symbiotic state (aposymbiotic, homologous, and heterologous states) including molecular chaperones and ubiquitin proteins. Furthermore, many of the differentially-expressed genes and metabolites identified were similar to those previously identified between symbiotic and aposymbiotic cnidarians, including stress response genes such as TNF receptors involved in apoptosis, and metabolite transport and cycling genes including the sodium/glucose cotransporter SGLT4 (Lehnert et al. 2014). The intermediate profiles therefore suggests that the presence of heterologous symbionts affects common molecular processes involved in the coraldinoflagellate symbiosis, and contributes to evidence that modulation of host gene expression plays a role in the establishment and maintenance of the functional symbiosis (Voolstra et al. 2009; DeSalvo et al. 2010b; Schnitzler and Weis 2010; Mohamed et al. 2016). This is similar to the regulation of host gene expression, including basal cellular processes, in the obligate symbiosis between Euprymna scolopes and the bioluminescent bacteria Vibrio fischeri (Rader and Nyholm 2012). 
Stress response processes also likely play a role in host recognition, symbiont tolerance mechanisms, and communication between the partners (Davy et al. 2012). The importance of such processes was suggested in the current study by the large number of stress-related genes and metabolites that were differentially expressed between the three symbiotic states. Furthermore, a large number of these stress-related proteins were involved in redox homeostasis and apoptosis mechanisms. Many of these genes, including the autophagic enzyme caspase-8 (Yu et al. 2004; Dunn et al. 2007; Hawkins et al. 2013), were downregulated in the homologous Symbiodinium B1-colonised hosts relative to the two other symbiotic states. A similar pattern of expression of caspase enzymes was observed in Fungia scutaria when exposed to heterologous symbionts (Dunn and Weis 2009). Collectively, this supports a role for immune response attenuation or avoidance mechanisms in the successful establishment of the cnidarian-dinoflagellate symbiosis (Rodriguez-Lanetty et al. 2006a; Voolstra et al. 2009; Schnitzler and Weis 2010; Lehnert et al. 2014; Mohamed et al. 2016).

\subsubsection{Evidence for the catabolism of host energy stores in a heterologous association}

The results demonstrated that, despite the similarity in the autotrophic potential when hosting Symbiodinium B1 and D1a, the D1a-colonised hosts experienced reduced glycogen synthesis and increased catabolism of lipid stores. Given that fixed carbon translocated to the host accumulates in glycogen deposits and lipid droplets in coral tissues (Kopp et al. 2015), the results suggest that modifications to the abundance and identity of translocated carbon occurs in the Dla-colonised hosts. Similarly, previous studies have shown that Symbiodinium genotypic diversity affects the identity and quantity of material translocated to the host (Loram et al. 2007; Stat et al. 2008; Starzak et al. 2014; Leal et al. 2015; Pernice et al. 2015). For instance the sea anemone host Condylactis gigantea incorporates less photosynthetic carbon from clade B symbionts into host amino acid and lipid pools compared to conspecifics harbouring clade A symbionts (Loram et al. 2007). More specifically, heterologous association with clade D symbiont in Aiptasia revealed lower carbon translocation than conspecifics harbouring homologous types (Leal et al. 2015). These findings agree with prior evidence that hosts harbouring members of clade D (particularly type Dla) experience reduced carbon translocation (Cantin et al. 2009), or altered photokinetics and energetics (Jones and Berkelmans 2011; Jones and Berkelmans 2012), leading to slower coral growth (Little et al. 2004; Jones and Berkelmans 2010; Cunning et al. 2015a).

Notably, there was a greater relative abundance of free sugars in the D1a-colonised host pools, however pathway activity identified a reduction in the conversion of sugars to glycogen stores. 
Glycogen is an important form of energy storage in coral tissues (Hosoi 1938; Hayes and Goreau 1977; Kopp et al. 2015), and the results show that glycogen metabolism serves as a proxy for the functional state of the symbiosis. Similar reductions in glucan-branching enzyme (GBE1) activity involved in glycogen synthesis have been observed during dysbiosis in the coral Acropora palmata under thermal stress conditions (DeSalvo et al. 2010a). Moreover, multiple sugar transporters that were hypothesised by Lehnert et al (2014) to be involved in photosynthate translocation were downregulated in the D1a-colonised hosts, including three myo-inositol co-transporters and two sodium glucose transporters. Although the origin of the sugars cannot be determined from the methods used, reduction in glycogen storage was perhaps a result of reduced translocation of photosynthate from the symbiont to host, or reduced availability of inorganic carbon substrates for photosynthesis. Supporting this latter point, a glycosylphosphatidylinositol-linked carbonic anhydrase (GPI-CA), which may play a role in dissolved inorganic carbon translocation (Moya et al. 2008), was downregulated in the D1a-colonised hosts.

Similarly, the results reveal that the mobilisation of stored lipids and fatty acids was required by the host to support its central metabolism when harbouring Symbiodinium D1a, perhaps because of either a reduction or absence in the translocation of the required fatty acids and lipogenesis precursors from the Dla symbiont. This complements evidence that host lipid content, and the identity and abundance of translocated fatty acids are also influenced by symbiont type (Zhukova and Titlyanov 2003; Cooper et al. 2011a; Cooper et al. 2011b). For instance, Symbiodinium isolated from Pocillopora damicornis and Seriatopora caliendrum differed in the proportion of C18:4n-3 and C22:6n-3 (Zhukova and Titlyanov 2003). Moreover, fatty acids and phospholipids are prerequisites for the $G_{0} / G_{1}$ cell cycle phase of Symbiodinium, and are essential for anchoring Symbiodinium surface glycan involved in recognition of the symbionts (Wood - Charlson et al. 2006; Wang et al. 2013). Therefore the exchange of lipids and fatty acids has been suggested to be essential for the successful recognition and establishment of the endosymbiosis (Wang et al. 2013). Given that change in lipid and fatty acid composition has been shown to reflect changes in the nutrition, ecology and ultimately health of corals (Oku et al. 2003b; Saunders et al. 2006), endosymbiont type and novel associations may thus affect the long term survival of the holobiont.

Although the amount of carbon translocated by each symbiont type was not assessed here, the increased catabolism of stored energy in the presence of Symbiodinium D1a under the current experimental conditions suggests that increased host heterotrophy would be required to sustain this symbiosis to the same extent as the normal (i.e. B1-colonised) association (Loram et al. 
2007; Leal et al. 2015). Thus, the data not only support the importance of trophic plasticity for the survival of these associations (Grottoli et al. 2006; Leal et al. 2015), but also suggest that once host energy stores are exhausted, inadequate nutritional exchange between host and symbiont is likely to be a limiting factor for the maintenance of a novel symbiosis.

\subsubsection{Disruption of oxidative homeostasis}

Cellular responses vary with the dose of ROS experienced by cells: at lower levels, ROS have positive roles, initiating essential signalling cascades and promoting adaptations to new biotic or abiotic conditions, while at higher levels ROS can damage diverse cellular macromolecules, including proteins, lipids and DNA, arrest the cell cycle, and induce apoptosis (Lesser 2006; Ayer et al. 2014). Intricate control of the redox environment is therefore essential for the maintenance of active signalling pathways while avoiding pathogenic responses to oxidative stress, and features in the establishment and selection of specific partners in multiple mutualistic symbioses (Lesser 2006; Moné et al. 2014). For example, ROS regulation plays an important role in the tolerance of resident bacteria and elimination of pathogenic ones in Drosophila melanogaster (Ha et al. 2009). The results of the current study demonstrated a disruption of cellular oxidative homeostasis in the heterologous Symbiodinium D1a-colonised host, suggesting that the regulation of the oxidative environment is likewise an important process when establishing and maintaining a novel cnidarian-dinoflagellate associations (Moné et al. 2014). In particular, the production of oxidative-stress response proteins is consistent with an accumulation of ROS and RNS in the D1a-colonised host tissues. This observation is compatible with the known role of ROS and RNS in the cnidarian bleaching cascade (Weis 2008; Lesser 2011), as well as previous reports that the successful infection of Aiptasia with homologous symbionts results in a downregulation of host NO production pathways (Detournay et al. 2012), while infection of Aiptasia with unsuitable or dysfunctional symbionts leads to heightened host NO synthesis (Hawkins 2013). Furthermore, cnidarian host transcriptomic analysis has previously revealed that symbiont tolerance mechanisms likely involve complex changes to handle the production of ROS by the photosynthesising symbionts (Mohamed et al. 2016). Thus, the results support the involvement of similar mechanisms in determining the specificity and collapse of the cnidarian dinoflagellate symbiosis.

More specifically, the analysis revealed a higher abundance in D1a-colonised hosts of multiple enzymatic antioxidants, such as peroxidases, catalases and enzymes involved in glutathione production, and non-enzymatic antioxidants, such as uric acid and the MAAs tyrosine and derivative tyramine (Dunlap and Yamamoto 1995; Kim et al. 2001; Shick and Dunlap 2002; 
Rosic and Dove 2011). Selection of the luminescent bacterium Vibrio fischeri in the colonisation of the light organ of the bob-tail squid is a result of RNS detoxifying enzymes produced by only competent bacteria (Davidson et al. 2004), and such antioxidant provisioning, either by a novel Symbiodinium type and/or by the host in response to colonisation by a novel symbiont type, may similarly provide a mechanism for preventing symbiosis breakdown, and allowing for the establishment of novel cnidarian-dinoflagellate symbioses (Moné et al. 2014). Elevated antioxidant defences in D1a-colonised hosts could enhance the thermo- and/or oxidative-tolerance of the holobiont, perhaps helping to explain the stress resilience conferred by this symbiont type (Berkelmans and Van Oppen 2006; Stat and Gates 2011). While the origin of the host antioxidants in this study (i.e. host or symbiont), could not be determined from the methods used, it certainly seems plausible that the enhanced host antioxidant pools seen in response to Symbiodinium Dla may explain the ability of this heterologous symbiont type to form a functional symbiosis with Aiptasia, despite the other potentially negative functional consequences observed (e.g. nutritional cost).

\subsubsection{Molecular signalling pathways}

When ROS overwhelm the antioxidant capacity of an organism, a signalling cascade is initiated via G protein-coupled receptors (GPCRs). GPCRs are involved in signalling pathways for various cellular responses such as the modulation of host gene expression (Peng et al. 2010). Moreover, GPCRs have recently been identified in the symbiosomes isolated from Aiptasia pallida (Peng et al. 2010), and so could play a pivotal role in the molecular transport and intimate cross-talk between the partners. For instance, GPCRs are important in phosphatidylinositol signalling pathways, which were upregulated in the D1a-colonised anemones. Furthermore, there was a difference in the abundance and transport of inositol isoforms between the B1- and D1a-colonised hosts. Inositol has highly conserved roles in cellular recognition, signalling and development, and is reportedly translocated between the symbiont and cnidarian host (Burriesci et al. 2012; Hillyer 2016). Moreover, inositol and inositol-phosphates are thought to participate in apoptosis and autophagy - important mechanisms in symbiosis regulation (Dunn et al. 2004; Dunn et al. 2007; Weis 2008; Hillyer 2016). Interestingly, the abundance of inositol isoforms is distinctive between Symbiodinium types and is strongly affected by changes in temperature (Klueter et al. 2015). My results therefore support an important signalling role for inositol in symbiosis establishment and maintenance. Going forward, it will be of interest to explore how symbiont-derived metabolites, particularly inositol compounds, feature in the molecular signalling pathways of the cnidarian host. 
Enzymatic or non-enzymatic oxidation of 20-carbon long PUFAs, like arachidonic acid (C20:4), produces eicosanoid signalling molecules that function in diverse physiological and pathological systems, and in the cnidarian-dinoflagellate system have been reported to play a key role in coral immunity, initiating the caspase-3 apoptosis pathway (Savchenko et al. 2010; Libro et al. 2013; Lõhelaid et al. 2015). The results show an upregulation of C20:4 and cytochrome enzymes that catalyse C20:4 to signalling derivatives (Lesser 2006). Simultaneously upregulated was acyl-CoA synthetase long-chain family member 4 (ACSL4), which is distinguished by its preference for metabolising ARA into arachidonoyl-CoA. If arachidonoyl-CoA is subsequently inserted into lipid membranes and oxidised due to cellular oxidative stress, ferroptosis cell death is induced (Magtanong et al. 2016). Nevertheless, I also observed a significantly higher abundance of dihomo- $\gamma$-linolenic acid (DGLA) (C20:3) in D1acolonised hosts, which acts as an antagonist of the ARA leukotriene-signalling cascade, serving to counter the inflammatory effects of ARA. DGLA is a product of elongation of C18:3n-6, which in turn is a product of desaturation of C18:2n-6, the latter of which has been previously suggested to be a symbiont-only ability (Dunn et al. 2012); both of which were significantly more abundant in D1a-colonised host tissues. Collectively, this indicates that eicosanoid signalling is active in the Dla-colonised hosts, which may indicate an alternative mechanism to the caspase signalling pathway identified in Fungia scutaria larvae for executing apoptosis in response to a novel symbiont type (Dunn and Weis 2009). These various stressrelated metabolites provide interesting biomarkers for future investigations into cellular signalling mechanisms in the cnidarian-dinoflagellate symbiosis.

\subsection{Conclusion}

The results from this study highlights the power of statistical integration of 'omics' datasets in providing a greater understanding of the cellular processes in action (Cavill et al. 2015). Integrated pathway analysis is a hypothesis driving technique, providing new avenues for research and potential biomarkers for further investigation. Here, it has provided novel information about the molecular processes involved symbiosis specificity in the coraldinoflagellate symbiosis, in which nutrient exchange and modulation of cellular oxidative status may allow for the formation and persistence of evolutionary novelties in the cnidariandinoflagellate symbiosis. Nevertheless, it should be recognised that these responses were observed under the optimal growth conditions for the homologous Symbiodinium-Aiptasia association, which may not be the optimal conditions for the heterologous D1a association. 
Furthermore, there remain unanswered questions regarding whether the sugars, lipids, fatty acid, proteins, and amino acids are host derived or symbiont derived; such information will help to further resolve the complex biological mechanisms of the symbiosis. Stable isotope labelling will allow us to trace their origin (Hiller et al. 2010; Burriesci et al. 2012). 


\section{CHAPTER 4}

Symbiont type affects the identity and quantity of organic carbon translocation and metabolism: Implications for partner switching in the cnidarian-dinoflagellate symbiosis

\subsection{Introduction}

Metabolic interactions in the symbiosis between reef-building corals and diverse dinoflagellates in the genus Symbiodinium allow for the persistence and growth of highly productive coral reef ecosystems in nutrient-limited waters (Muscatine and Porter 1977). The symbionts are hosted within cnidarian gastrodermal cells, and are separated from the host cytoplasm by a perisymbiotic ("symbiosome") membrane of both host and algal origin (Wakefield et al. 2000; Wakefield and Kempf 2001), which is involved in controlling metabolic exchange between the partners (Rands et al. 1993; Peng et al. 2010). The coral host contributes dissolved inorganic carbon, nitrogen, phosphorus, as well as amino acids, lipids and fatty acids (Wang and Douglas 1999; Imbs et al. 2014), and a fixed position in the water column for the endosymbionts, enabling photosynthesis and production of organic carbon via the Calvin-Benson "C3" photosynthetic pathway (Davy et al. 2012). Studies with radio- and stable-isotope labelled carbon and nitrogen tracers revealed that the Symbiodinium translocate a portion of the organic products to the coral host, contributing to host respiration, growth calcification and reproduction (Muscatine and Hand 1958; Tanaka et al. 2006; Burriesci et al. 2012; Pernice et al. 2012; Kopp et al. 2015). Symbiont-derived metabolites identified in coral tissues include soluble, low molecular weight compounds such as glucose, glycerol, and amino acids including alanine, leucine and tyrosine (Trench 1971b; Wang and Douglas 1999; Whitehead and Douglas 2003; Burriesci et al. 2012; Hillyer et al. 2016a), to more complex molecules such as fatty acids, organic acids, and lipids (Von Holt and Von Holt 1968b; Papina et al. 2003; Whitehead and Douglas 2003; Grottoli et al. 2004; Baumann et al. 2014; Kopp et al. 2015; Hillyer 2016; Hillyer et al. 2016a).

Taxonomic diversity within the Symbiodinium genus has resulted in a nomenclature of cladal designations (A-I; with multiple sub-clades, or "types"), and this diversity is reflected in variance in the ecology and physiology of the symbionts, including thermal tolerance and photosynthetic carbon flux (Berkelmans and Van Oppen 2006; Loram et al. 2007; Starzak et al. 2014; Leal et al. 2015). Thus, the adaptive capacity of corals to environmental perturbations such as thermal bleaching events, is not only a result of the host response, but also of the 
genotype(s) of the associated symbionts (Blackall et al. 2015). Plasticity in corals' symbiotic associations, i.e. their capacity to associate with different Symbiodinium partners that are better adapted to different environmental conditions, may allow corals to acclimatise to changing ocean conditions, yet many corals form associations with specific algal genotypes (Baker 2003; Berkelmans and Van Oppen 2006; Loram et al. 2007; Silverstein et al. 2012; Blackall et al. 2015). The capacity to change the resident symbionts suggests two potential adaptive mechanisms to environmental change: either dynamic shifts in the in hospite Symbiodinium community composition (symbiont 'shuffling'), or the establishment and persistence of a novel symbiosis with algal symbionts acquired anew from the surrounding seawater (symbiont 'switching') (Buddemeier and Fautin 1993; Fautin and Buddemeier 2004). The potential for the formation and perpetuation of a novel partnership is believed to be restricted to specific Symbiodinium genotypes, coral species and life stages, symbiont acquisition strategies, environmental conditions, or a combination of these variables (Goulet 2006; Coffroth et al. 2010; Kemp et al. 2014; Boulotte et al. 2016). However, recent fine-scale genotyping of an adult coral following a series of consecutive bleaching events demonstrated that the acquisition of a new Symbiodinium type from the surrounding environment is possible for adult corals (Boulotte et al. 2016).

The successful establishment of a novel symbiosis is likely to require multiple molecular processes, such as inter-partner recognition followed by a 'winnowing' mechanism (Nyholm and McFall-Ngai 2004; Wood - Charlson et al. 2006; Dunn and Weis 2009; Davy et al. 2012). Once compatible symbionts are enveloped in the symbiosome membrane, the establishment of a functional symbiosis likely requires a physiological and hence ecological benefit to the holobiont (Berkelmans and Van Oppen 2006; Jones et al. 2008). For example, the persistence of a novel symbiosis will likely require sufficient nutrient cycling between the partners in order to maintain functional cellular homeostasis (Chapter 3), and a viable symbiosis under the prevailing environmental conditions (Loram et al. 2007). In this regard, Symbiodinium metabolites vary in quantity and identity by genotype when in culture, and their metabolite profiles have been found to be affected by thermal stress (Klueter et al. 2015). These differences in metabolite profiles may extend to the compounds translocated to the cnidarian host (Trench 1979; Miralles et al. 1989; Bil et al. 1991; Loram et al. 2007; Starzak et al. 2014). Furthermore, the amount of carbon translocated to the host has been shown to depend on the resident symbiont (Trench 1979; Miralles et al. 1989; Bil et al. 1991; Loram et al. 2007; Cantin et al. 2009; Starzak et al. 2014; Leal et al. 2015). In particular, corals acquire less carbon from members of Symbiodinium clade D, in comparison to conspecifics harbouring other clades 
under normal conditions (Cantin et al. 2009; Leal et al. 2015), leading to slower coral growth (Little et al. 2004; Jones and Berkelmans 2010; Cunning et al. 2015a). However, the consequences of switching to a heterologous Symbiodinium genotype in terms of the identity of translocated photosynthetic carbon metabolites are currently unknown.

Here, I combine stable isotope labelling and gas chromatography-mass spectrometry to determine the identity and quantity of photosynthetic compounds transferred from multiple Symbiodinium genotypes to the model sea anemone host Aiptasia sp. Exposure to ${ }^{13} \mathrm{C}$-enriched media under normal photosynthetic conditions will result in the fixation of ${ }^{13} \mathrm{C}$-enriched carbon products in symbiont intracellular pools (Hillyer 2016), resulting in a variety of ${ }^{13} \mathrm{C}$-enriched metabolic intermediates and end-products. These metabolites may be used in symbiont metabolism or biosynthesis, or transferred to the host (Whitehead and Douglas 2003; Burriesci et al. 2012; Dunn et al. 2012; Kopp et al. 2015; Hillyer 2016). Consequently, the detection and quantification of ${ }^{13} \mathrm{C}$-enriched compounds in the host tissues can then be assumed to be a product of direct translocation or downstream metabolism of photosynthetically-fixed compounds translocated from symbiont to host. Presence of isotopic enrichment in detected metabolites can be identified by characteristic shifts in the masses of corresponding ions in individual compounds, and can be used to quantitatively estimate the translocation of specific metabolites (Burriesci et al. 2012; Hiller et al. 2013; Weindl et al. 2015; Hillyer 2016; Weindl et al. 2016a; Weindl et al. 2016b).

The focus of this chapter was to determine the effect of symbiont-type on the identity and quantity of translocated carbon compounds, and how the patterns of translocation relate to symbiosis specificity. The simultaneous identification and quantification of isotopically labelled metabolites was investigated in Aiptasia experimentally colonised with either homologous symbiont S. minutum (referred to in this study by its ITS2 type B1), or heterologous S. trenchii (ITS2 type D1a). The potential of D1a to provide resilience and assist in host recovery makes it an especially interesting candidate for investigations into the nutritional implications of partner switching (Berkelmans and Van Oppen 2006; Stat and Gates 2011). The findings from this study contribute to our understanding of symbiont-type effects on metabolite translocation and downstream metabolism, and hence the adaptive potential of the symbiosis. 


\subsection{Materials and Methods}

\subsubsection{Experimental organism}

Symbiotic Aiptasia sp. $(n \sim 750)$ originally collected from Hawaii were harvested from a longterm laboratory stock and maintained in $0.22 \mu \mathrm{m}$ aerated filtered seawater (FSW). Temperature was maintained at $25{ }^{\circ} \mathrm{C}$ with light provided by AQUA-GLO T8 fluorescent bulbs at $\sim 95 \mu \mathrm{mol}$ photons $\mathrm{m}^{-2} \mathrm{~s}^{-1}$ (light:dark $=12 \mathrm{~h}: 12 \mathrm{~h}$ ). Anemones were fed twice a week with freshly hatched Artemia sp. nauplii.

\subsubsection{Experimental design}

Anemones were rendered aposymbiotic following the menthol-bleaching procedure (Chapter 2). Whole anemones $(n=20)$ from the bleaching treatment were examined by confocal microscopy (Olympus Provis AX70, at 100× magnification) to detect the chlorophyll autofluorescence of any residual dinoflagellates. Anemones were selected at random and placed in a 'relaxation solution' [50\% FSW, 50\% $0.37 \mathrm{~mol}^{-1}$ magnesium chloride $\left(\mathrm{MgCl}_{2}\right)$ ] for 15 min before being moved to a FluoroDishTM glass-bottom confocal dish (World Precision Instruments, FL, USA). The autofluorescence emission of the Symbiodinium cells was excited using a $559 \mathrm{~nm}$ laser and captured at $647 \pm 10 \mathrm{~nm}$. This confirmed the aposymbiotic state of the anemones.

Two different Symbiodinium cultures were used, comprised of the homologous type $S$. minutum (ITS2 type B1) originally isolated from laboratory Aiptasia stock, and a heterologous type S. trenchii (D1a, culture ID Ap2). Symbiodinium cells were sub-cultured from a laboratory stock and grown in silica-free F/2 medium (AusAqua Pty, SA, Australia) for 6 weeks before use in the colonisation study. The cultures were maintained at $25{ }^{\circ} \mathrm{C}$ with light provided by cool white fluorescent lamps (Phillips, 18W 840), at an irradiance of $100 \mu \mathrm{mol}$ photons $\mathrm{m}^{-2} \mathrm{~s}^{-1}$ with a $12 \mathrm{~h}: 12 \mathrm{~h}$ light:dark photoperiod.

\subsubsection{Symbiont colonisation of aposymbiotic Aiptasia}

Aposymbiotic anemones ( $n \sim 240$ per treatment) were divided into $12 \times 250 \mathrm{~mL}$ plastic sterile beakers (LabServ, Auckland, New Zealand) each with 20 anemones, containing $200 \mathrm{~mL}$ of $0.22 \mu \mathrm{m}$ aerated FSW. Anemones were starved for seven days prior to colonisation. One drop of the Symbiodinium cells at $\sim 3 \times 10^{6}$ cells $\mathrm{mL}^{-1}$ was pipetted onto the oral disc of each anemone (except for the aposymbiotic treatment where a drop of $0.22 \mu \mathrm{m}$ FSW only was used), followed by a dilute suspension of Artemia sp. nauplii to enhance phagocytosis (Davy et 
al. 1997). The aposymbiotic anemones were exposed to Symbiodinium cells for $24 \mathrm{~h}$ before the $0.22 \mu \mathrm{m}$ FSW was changed. This colonisation procedure was repeated weekly for 3 more weeks (i.e. 4 weeks in total), during which the temperature and light regimes were maintained as per pre-colonisation.

\subsubsection{Measurement of $\mathrm{O}_{2}$ flux}

On the sampling day, maximum photosynthetic and dark respiratory $\mathrm{O}_{2}$ fluxes were measured to compare the symbiosis functionality of anemones colonised by homologous type B1 and heterologous type D1a symbionts as per the methods of Starzak et al. (2014). Briefly, individual anemones ( $n=4$ per treatment each sampled from different sample containers) were placed in a glass chamber of volume $10 \mathrm{~mL}$, itself situated in a glass water bath set at $25^{\circ} \mathrm{C}$. The chamber contained $0.22 \mu \mathrm{m}$ FSW stirred via a magnetic spin bar that rotated the medium speed at which the anemones did not appear stressed. A perforated nylon mesh overlaid the stir bar, on which the anemone was placed and allowed to settle for $1 \mathrm{~h}$ prior to oxygen measurements so that it could attach to the mesh and open its tentacles. The chamber was sealed by a glass lid with a rubber O-ring, into which an oxygen electrode (FIBOX 3 fibreoptic oxygen meter; PreSens GmbH, Germany) and temperature probe were inserted. These were connected to the oxygen meter and were used to measure the respiration rate $\left(\mathrm{R}, \mathrm{mL} \mathrm{O}_{2}\right.$ $\mathrm{h}^{-1}$ ) for $30 \mathrm{~min}$ in the dark, by which time a constant rate was observed. The oxygen tension was not allowed to fall below 50\% saturation (Davies 1984). Subsequently, the rate of net photosynthesis was measured for $30 \mathrm{~min}$ at an irradiance of $400 \mu \mathrm{mol}$ photons $\mathrm{m}^{-1} \mathrm{~s}^{-1}$ (lightsaturation for photosynthesis (Starzak et al. 2014)). Light was provided by a Thorn PAR (photosynthetically active radiation) $38150 \mathrm{~W}$ sealed beam-reflector lamp and irradiance on the surface of the chamber was measure by a QSL-100 irradiance meter (Biospherical Instruments Inc.). Net photosynthesis and respiration were corrected for seawater microbial activity using a $0.22 \mu \mathrm{m}$ FSW-only control, and gross photosynthesis ( $\left.\mathrm{P}_{\text {gross }}\right)$ was calculated by the addition of gross photosynthesis to dark respiration (Muscatine et al. 1981).

After the $\mathrm{O}_{2}$-flux measurements were complete, individual anemones were then placed in a 1.5-mL microcentrifuge tube and homogenised in $1 \mathrm{~mL} 0.22 \mu \mathrm{m}$ FSW using a plastic tissue grinder until fully homogenised, and the homogenate then centrifuged at $1,000 \times g$ for 5 min to separate the animal and algal fractions. The animal supernatant was collected, and the algal pellet re-suspended in $1 \mathrm{~mL}$ FSW. Symbiodinium cell densities for $\mathrm{O}_{2}$-flux normalisation were quantified from the algal fraction using Improved Neubauer haemocytometer counts (Boeco, Germany) with a minimum of ten replicate counts per sample (i.e. to a confidence interval 
below 10\%). Cell density was normalised to soluble host protein content of the host fraction, which was assessed by the Bradford assay (Bradford 1976) and bovine-serum albumin (BSA) as the standard. The total gross photosynthetic and respiration rates were adjusted for the respiration chamber volume and normalised to host protein content, while gross photosynthesis was also normalised to algal cell density. The average ratio of gross photosynthesis to respiration $\left(\mathrm{P}_{\text {gross }}: \mathrm{R}\right)$ for $24 \mathrm{~h}$ was calculated for each anemone, by adopting a $12 \mathrm{~h}: 12 \mathrm{~h}$ light:dark cycle and that respiration was constant in the light and dark, providing a proxy for autotrophic potential. Data were assessed for normality and homoscedasticity were assessed using Shapiro-Wilk and Levene's tests, respectively, and a Student $t$-test performed at the $p<$ 0.05 probability level to compare $\mathrm{R}, \mathrm{P}_{\text {gross, }}$, and $\mathrm{P}_{\text {gross }}: \mathrm{R}$ between treatments, using SPSS statistical software (v. 20, IBM Corp).

\subsubsection{Stable isotope labelling and sampling}

Stable isotope labelling was conducted four weeks after initial infection, and anemones were starved for one week prior to labelling. Of the 12 beakers for each treatment, six beakers contained ${ }^{12} \mathrm{C}$-enriched $0.22 \mu \mathrm{m}$ FSW and six contained ${ }^{13} \mathrm{C}$-enriched FSW). At the beginning of the light cycle on the day of sampling $(08: 00 \mathrm{H})$, the $0.22 \mu \mathrm{m}$ FSW was replaced with either

$\mathrm{H}^{12} \mathrm{CO}_{3}{ }^{-}$-or $\mathrm{H}^{13} \mathrm{CO}_{3}{ }^{-}$-enriched $\mathrm{FSW}$ to a final concentration of $4 \mathrm{mM}$ representing a bicarbonate enrichment of approximately 66\% (Tremblay et al. 2012), and buffered to the original seawater $\mathrm{pH}$ of 8.2 with $\mathrm{NaOH}(1 \mathrm{M})$. Samples were incubated for $5 \mathrm{~h}$ under the same light (AQUA-GLO T8 fluorescent bulbs at $\sim 95 \mu \mathrm{mol}$ photons $\mathrm{m}^{-2} \mathrm{~s}^{-1}$ ) and temperature regime $\left(25^{\circ} \mathrm{C}\right)$, to allow for sufficient photosynthetic activity and incorporation of the relevant carbon label, based on methods by Hillyer (2016). Samples were removed from the water bath, the FSW gently discarded, and the beakers sealed and flash frozen in liquid nitrogen to quench their metabolism $(\sim 10 \mathrm{~s})$. Samples were stored at $-80{ }^{\circ} \mathrm{C}$ until processing.

\subsubsection{Metabolite profile sample host-symbiont separation}

All sample-processing steps were performed at $4{ }^{\circ} \mathrm{C}$. Frozen anemones were moved from sample beakers to $15 \mathrm{~mL}$ falcon tubes, and $500 \mu \mathrm{L}$ of cold $\left(4{ }^{\circ} \mathrm{C}\right) \mathrm{MilliQ}$ water was added. Anemones were rapidly homogenised with a mechanical saw-tooth homogeniser (IKA T10 BS5, ThermoFisher Scientific, Auckland, New Zealand) for $1 \mathrm{~min}$ at a mid-speed setting. Once homogenised, $5 \mathrm{~mL}$ of cold $\left(4^{\circ} \mathrm{C}\right)$ MilliQ water was added, the sample thoroughly vortexed and a single $500 \mu \mathrm{L}$ aliquot removed for host protein content and Symbiodinium cell density estimates (Section 4.2.7.). Symbionts were separated by centrifugation $(2,500 \mathrm{x} g$ for $5 \mathrm{~min}$ at $4^{\circ} \mathrm{C}$ ), and the host supernatant transferred to a new $15 \mathrm{~mL}$ falcon tube. Both fractions were 
diluted with $5 \mathrm{~mL}$ of cold MilliQ water, followed by vigorous vortexing for $1 \mathrm{~min}$ and a second centrifugation $\left(2,500 \times \mathrm{g}\right.$ for $5 \mathrm{~min}$ at $\left.4^{\circ} \mathrm{C}\right)$. The host fraction was moved to a new $15 \mathrm{~mL}$ falcon tube, and the residual supernatant on the symbiont pellet discarded. Subsamples $(n=20)$ of the host material were confirmed for Symbiodinium absence by light microscopy (at x40 magnification). The cleaned host fractions were frozen at $-80{ }^{\circ} \mathrm{C}$ for $1 \mathrm{~h}$ and lyophilised (FreeZone ${ }^{\circledR}$ Plus ${ }^{\mathrm{TM}} 4.5 \mathrm{~L}$ Cascade, Labconco, Auckland, New Zealand) for $18 \mathrm{~h}$ at $-105^{\circ} \mathrm{C}$. To the symbiont pellets, $3 \mathrm{~mL} 500 \mu \mathrm{L}$ of cold $\left(4{ }^{\circ} \mathrm{C}\right)$ MilliQ water was added, the sample thoroughly vortexed and a single $500 \mu \mathrm{L}$ aliquot transferred to a $2 \mathrm{~mL}$ microcentrifuge tube (Eppendorf, Lab, Interlab, Auckland, New Zealand). Then $500 \mu \mathrm{L}$ of DNA buffer $(0.4 \mathrm{M}$ $\mathrm{NaCl}, 0.05 \mathrm{M}$ EDTA) with $1 \%(\mathrm{w} / \mathrm{v})$ sodium dodecyl sulphate (SDS) was added, and the samples frozen at $-20^{\circ} \mathrm{C}$ for symbiont identification (Section 4.2.8.). The remaining symbiont material was frozen at $-80^{\circ} \mathrm{C}$.

\subsubsection{Symbiont density and protein quantification}

The aliquot of homogenate (Section 4.2.6.) was centrifuged at $1,000 \times \mathrm{g}$ for $5 \mathrm{~min}$ to separate the animal and algal fractions. The host supernatant was collected, and the algal pellet resuspended in $1 \mathrm{~mL}$ FSW. Symbiodinium cell densities were quantified for metabolite profile normalisation using Improved Neubauer haemocytometer counts (Boeco, Germany) with a minimum of ten replicate counts per sample (i.e. to a confidence interval below $10 \%$ ). Cell density was normalised to soluble protein content of the host supernatant, which was assessed by the Bradford assay (Bradford 1976) and BSA as the standard. To test for differences in Symbiodinium cell densities between the treatments groups, assumptions of normality and homoscedasticity were assessed using Shapiro-Wilk and Levene's tests, respectively, and nonnormal or heteroscedastic data were $\log _{10}$-transformed prior to analysis with Student $t$-tests at the $p<0.05$ probability level using SPSS statistical software (v. 20, IBM Corp).

\subsubsection{Symbiont identification}

Symbiont type of each biological replicate was verified by direct sequencing of two loci that are known to be highly divergent between clades: internal transcribed spacer region 2 of ribosomal DNA (ITS2 rDNA) and chloroplast 23S rRNA (cp23S) (Ladner et al. 2012). DNA was extracted using the CTAB-chloroform extraction protocol (dx.doi.org/10.17504/protocols.io.dyq7vv). Samples were immediately analysed via PCR, using a published thermal cycling regime and reaction mixture (Hill et al. 2014), with the ITS2 primers ITSintfor2 and ITS2rev2, and cp23s primers cp23s1 and cp23s2 (sequences provided in Section 3.2.13). PCR products were cleaned with ExoSAP-IT (USB Corporation, OH, USA) 
and sequenced by Macrogen (Seoul, South Korea). Sequences were aligned with Geneious v. 7.0 (Biomatters, Auckland, NZ), and a BLAST search was carried out against Symbiodinium ITS2 and cp23s sequences in GenBank.

\subsubsection{Intracellular metabolite extraction}

Extraction was based on the methods in Hillyer et al. (2016). Once thoroughly dry, $1 \mathrm{~mL}$ of $100 \%$ methanol $\left(-20{ }^{\circ} \mathrm{C}\right)$ containing the internal standard D-sorbitol-6- ${ }^{13} \mathrm{C}$ (Sigma Aldrich, Auckland, New Zealand) was added to $30 \mathrm{mg}$ of lyophilised host material to extract the semipolar metabolites. The host fractions were sonicated for $30 \mathrm{mins}$ at $4{ }^{\circ} \mathrm{C}$ in an ultrasonic bath (model: 8854, Cole Parmer, USA) and centrifuged (3,000 x $\mathrm{g}$ for $30 \mathrm{~min}$ at $4^{\circ} \mathrm{C}$ ), and the supernatant containing the extracted metabolites was collected and stored at $-80^{\circ} \mathrm{C}$. Extraction was then repeated with $1 \mathrm{~mL}$ of $50 \% \mathrm{MeOH}\left(-20^{\circ} \mathrm{C}\right)$ to extract the polar fraction. Samples were then centrifuged $\left(3,000 \times \mathrm{g}\right.$ for $30 \mathrm{~min}$ at $\left.4^{\circ} \mathrm{C}\right)$ and the supernatant pooled with the first extract. The total extract was then further centrifuged at $16,100 \times \mathrm{g}$ for $15 \mathrm{~min}$ at $4^{\circ} \mathrm{C}$ to ensure the removal of all particulates. Aliquots (total volume $200 \mu \mathrm{L}$ ) were then concentrated under vacuum (Eppendorf Concentrator 5301, Global Science, Auckland) at $30{ }^{\circ} \mathrm{C}$ until dry. The cell debris was frozen at $-20^{\circ} \mathrm{C}$ for protein quantification as determined by a modified Bradford colorimetric method (Smart et al. 2010) with BSA as the standard (Bradford 1976).

Trimethylsilyl ester derivatisation of sugars, sugar alcohols and amino/non-amino acids for GC-MS was performed using an automated on-line method, adapted from established protocols (Roessner et al. 2000). Briefly, all samples were re-dissolved in $20 \mu \mathrm{L}$ of $30 \mathrm{mg} \mathrm{mL} \mathrm{m}^{-1}$ methoxyamine hydrochloride in pyridine and derivatised at $37{ }^{\circ} \mathrm{C}$ for $120 \mathrm{~min}$ with mixing at $500 \mathrm{rpm}$. Twenty microlitres N,O-bis-(trimethylsilyl)trifluoroacetamide (BSTFA) and $1 \mu \mathrm{L}$ retention-time standard mixture comprised of $n$-alkanes were then added and the samples incubated for a further $30 \mathrm{~min}$ with mixing at $500 \mathrm{rpm}$. Each derivatised sample was then allowed to rest for 60 min prior to injection into the GC-MS. GC-MS analysis was performed on an Agilent 7890 gas chromatograph equipped with Gerstel MPS2 multipurpose sampler and coupled to an Agilent 5975C VL mass selective detector, run in splitless mode, with an injection volume of $1 \mu \mathrm{L}$ of each sample and a technical replicate of two injections per sample. Instrument control was performed with Agilent G1701A Revision E.02.01 ChemStation software. The gas chromatograph was fitted with a Varian Factor 4 column (VF-5ms; $30 \mathrm{~m} \times$ $0.25 \mathrm{~mm} \times 0.25 \mathrm{um}+10 \mathrm{~m}$ Ezi-guard). Helium was in constant flow mode at approximately 1 $\mathrm{mL} \min ^{-1}$ with retention-time locking applied. The oven temperature was started at $70{ }^{\circ} \mathrm{C}$, held 
at this temperature for $1 \mathrm{~min}$ and then increased at $7{ }^{\circ} \mathrm{C} \min ^{-1}$ to $325^{\circ} \mathrm{C}$, and finally held at this temperature for $3.5 \mathrm{~min}$.

The mass spectrometer quadrupole temperature was set at $150^{\circ} \mathrm{C}$, with the source set at $250^{\circ} \mathrm{C}$ and the transfer line held at $280^{\circ} \mathrm{C}$. Positive ion electron impact spectra at $70 \mathrm{eV}$ were recorded in scan mode with the following settings: a gain factor of 1.00 , detector threshold of 150 , mass to charge $(\mathrm{m} / \mathrm{z})$ range of $50-600 \mathrm{~m} / \mathrm{z}$ and solvent delay of $6.0 \mathrm{~min}$. Along with the samples, a series of known metabolite mixtures and solvent-only blanks were analysed. The metabolite mixtures contained the major compound classes being targeted, including amino acids (Amino Acid Standard solution AAS18, Sigma-Aldrich, Auckland), fatty acids (Menhaden fish oil, Sigma-Aldrich, Auckland), and sugars $(2.5 \mu \mathrm{M}$ ribitol, ribose, fructose, glucose, arabinose, galactose, mannitol, xylose, mannose and sucrose; Sigma-Aldrich, Auckland), and were analysed to confirm that these compounds could be accurately identified.

\subsubsection{Metabolite data extraction, pre-processing and normalisation}

Metabolite data extraction and analysis were performed with the use of AMDIS (http://chemdata.nist.gov/mass-spc/amdis/) and Agilent MassHunter Workstation Software, Quantitative Analysis, Version B.05.00/Build 5.0.291.0. Compound identification was based on an in-house library of MS spectra, with retention indices based on n-alkane and the standard mixes. Derivative peak areas were used to semi-quantify the concentrations of individual metabolites. Data were normalised to both the final area of the IS D-sorbitol-6- ${ }^{13} \mathrm{C}$ and the cell debris resulting from the samples' intracellular metabolite extraction using a modified Bradford colorimetric method (Smart et al. 2010) (see Section 4.2.9). Data were then tested for normality and homogeneity and $\log _{2}$ transformed where necessary. Data were then autoscaled, and Principal Component Analysis (PCA) was performed using MetaboAnalyst 3.0 (http://www.metaboanalyst.ca) (Xia et al. 2015). Relative metabolite concentrations between the three symbiotic states were evaluated by PCA.

\subsubsection{Relative enriched metabolite abundance}

Labelled compounds were detected using the following software: Automated Mass spectral Deconvolution and Identification System (AMDIS), Metabolite Detector (MD), Non-Targeted Tracer Fate Detection (NTFD), and Mass Isotopolome Analyzer (MIA) (Hiller et al. 2009; Hiller et al. 2013). For each sample, spectral components were separated, detected and identified during deconvolution using MD, according to retention indices and alkane standard runs. Once identifications were confirmed against AMDIS and MassHunter results described 
above, the sample files were imported into NTFD for ${ }^{13} \mathrm{C}$-enrichment analysis. Stable isotopic carbon labelling leads to isotopic isomerism. Mass isotopomers are a group of isomers with the same nominal mass, and the relative abundance of the different mass isotopomers of a molecule is known as the mass isotopomer distribution (MID). The MID is therefore an indication of the isotopic enrichment of a metabolite (i.e. $\mathrm{M}+i$, where $i$ is the increase in nominal mass compared to the lightest isotopomer). The NTFD software detects and identifies isotopic enrichment (MID) by comparing the labelled and unlabelled chromatograms of each sample based on retention time and mass spectral identity (Hiller et al. 2013). The MID for every detected and labelled fragment ion was calculated using the strictest detection settings in NTFD, in order to minimise the generation of false positives (Hiller et al. 2013; Weindl et al. 2015). Compounds were considered enriched if at least two spectral fragments were detected under at least one experimental treatment, with a coefficient of MID determination of at least $\mathrm{R}^{2}=0.98$ and minimal enrichment of $\mathrm{M}+i \geq 0.05$. To identify any non-photoautotrophic label incorporation, $\mathrm{M}+i$ for the aposymbiosis treatment was analysed. Any compounds identified as labelled under aposymbiotic conditions were excluded from further analysis. All host metabolites that were labelled in the symbiotic but not aposymbiotic state were therefore assumed to be a translocated compound or downstream product of the translocated compounds. For each labelled compound, a characteristic ion was manually selected; this was typically the most abundant or closest to the heaviest ion (the ion with the greatest $\mathrm{m} / \mathrm{z}$ ). Compounds were considered enriched if label was present in at least three out of the six replicates. In the case of multiple TMS derivatives of the same metabolite, only the derivative with the largest peak area in the AMDIS and MassHunter results was used. Mean values and the standard error mean (S.E.M.) for each MID associated with this ion were then calculated for each labelled compound, taking into account natural ${ }^{13} \mathrm{C}$ isotope abundance (approximately $1.1 \%$ of the total ion distribution). For each enriched compound, the relative intensity of the non-enriched fraction $(\mathrm{M}+0)$ will decrease, with corresponding increases in labelled fractions $(\mathrm{M}+1, \mathrm{M}+$ $2, \ldots, \mathrm{M}+i)$. The sum of the total enrichment for each compound can therefore be represented as $1-\mathrm{M}+0$ (Hillyer 2016; Weindl et al. 2016b).

\subsubsection{Statistical analysis of relative enriched metabolite abundance}

The relative pool size of each compound between treatments was calculated for the unlabelled $\left(\mathrm{M}_{0}\right)$ and labelled $\left(\mathrm{M}_{i}\right)$ fractions, using the following equations:

$$
\begin{gathered}
\mathrm{M}_{0}=\text { Normalised metabolite abundance } \times(M+0) \\
\mathrm{M}_{\mathrm{i}}=\text { Normalised metabolite abundance } \times(1-\mathrm{M}+0)
\end{gathered}
$$


Subsequently, to account for variations in symbiont density, the normalised relative metabolite abundances were further normalised to symbiont density prior to calculating relative amounts of the unlabelled $\left(\mathrm{M}_{0}\right)$ and labelled $\left(\mathrm{M}_{i}\right)$ fractions. These data were used to support the differences in the relative pools sizes found to be significant. Assumptions of normality and homoscedasticity were assessed using Shapiro-Wilk and Levene's tests, respectively. Nonnormal or heteroscedastic data were $\log _{10}$-transformed prior to analysis with Student $t$-tests at the $p<0.05$ probability level using SPSS statistical software (v. 20, IBM Corp). Data that could not be adequately transformed were analysed using non-parametric Mann-Whitney $U$ rank-sum tests.

\subsubsection{Pathway visualisation}

As metabolically related compounds show very similar MIDs, it is possible to connect compounds with highly similar MIDs into a metabolic network. The Mass Isotopolome Analyser (MIA) software clusters isotopically enriched compounds based on their pairwise similarity in MID. A Needleman-Wunsch alignment was performed on the MIDs to minimise the absolute differences in relative mass isotopomer abundances using a gap penalty of 0.4 . Subsequently, the pairwise distances of all aligned MIDs were determined using the Canberra distance metric and normalised by the sum of the compounds' MID vectors. As MIDs are a sign of proximity to a metabolic network, compounds that have similar MIDs are considered to be connected and visualised in a metabolite pathway diagram. Visual exploration of the metabolites in the respective metabolic pathways was illustrated using Vanted (v2.6.2) (Junker et al. 2006; Rohn et al. 2012).

\section{3. $\quad$ Results}

\subsubsection{Colonisation success and $\mathrm{O}_{2}$ flux}

Aposymbiotic Aiptasia sp. were successfully colonised by either homologous type B1 or heterologous type D1a, however homologous Symbiodinium cell densities were significantly larger ( 1.5-fold) than heterologous symbiont densities (Table $4.1 ; t=2.362$, df $=10, p=$ 0.04). Nevertheless, holobiont respiration ( $\mathrm{R} ; t=-0.162$, $\mathrm{df}=4, p=0.879$ ), gross photosynthesis ( $\left.\mathrm{P}_{\text {gross }} ; t=1.614, \mathrm{df}=4, p=0.182\right)$, and the ratio of gross photosynthesis to respiration ( $\mathrm{P}_{\text {gross }}: \mathrm{R} ; t=0.574$, df $=4, p=0.597$ ), were not significantly affected by whether the association was homologous or heterologous (Table 4.1). 
Table 4.1. Mean values for symbiont cell density and oxygen measurements of homologous type B1 and heterologous type D1a colonised Aiptasia. Values represent data means \pm S.E.M $(n=6)$. Respiration $(\mathrm{R})$ gross photosynthesis (Pgross) and the ratio are described.

\begin{tabular}{lll}
\hline Variable & Homologous type B1 & Heterologous type D1a \\
\hline $\begin{array}{l}\text { Symbiont cell density }\left(\text { cells } \mathrm{mg}^{-1}\right. \\
\text { protein) }\end{array}$ & $11222405.5 \pm 1163722.7$ & $7287005.8 \pm 1192616.2$ \\
$\mathrm{R}\left(\mu \mathrm{g} \mathrm{O} 2 \mathrm{mg}^{-1}\right.$ protein $\left.^{-1}\right)$ & $0.0257 \pm 0.0145$ & $0.0287 \pm 0.0117$ \\
$\mathrm{P}_{\text {gross }}\left(\mu \mathrm{g} \mathrm{O} 2 \mathrm{mg}^{-1}\right.$ protein $\left.^{-1}\right)$ & $0.0237 \pm 0.0081$ & $0.0097 \pm 0.0032$ \\
$\mathrm{P}_{\text {gross }}: \mathrm{R}$ & $1.249 \pm 0.2683$ & $0.976 \pm 0.3017$ \\
\hline
\end{tabular}

\subsubsection{Alterations in host free metabolite pools induced by symbiotic state}

Non-targeted GC-MS identified 81 metabolites in the Aiptasia host tissue extracts (Table S4.1). Principle component analysis (PCA) revealed a distinct spatial separation of the symbiotic states, with the heterologous Dla-colonised cluster intermediate between the aposymbiotic and homologous B1-colonised clusters along PC axis 1 (37.6\% of the explained variation) (Figure 4.1).

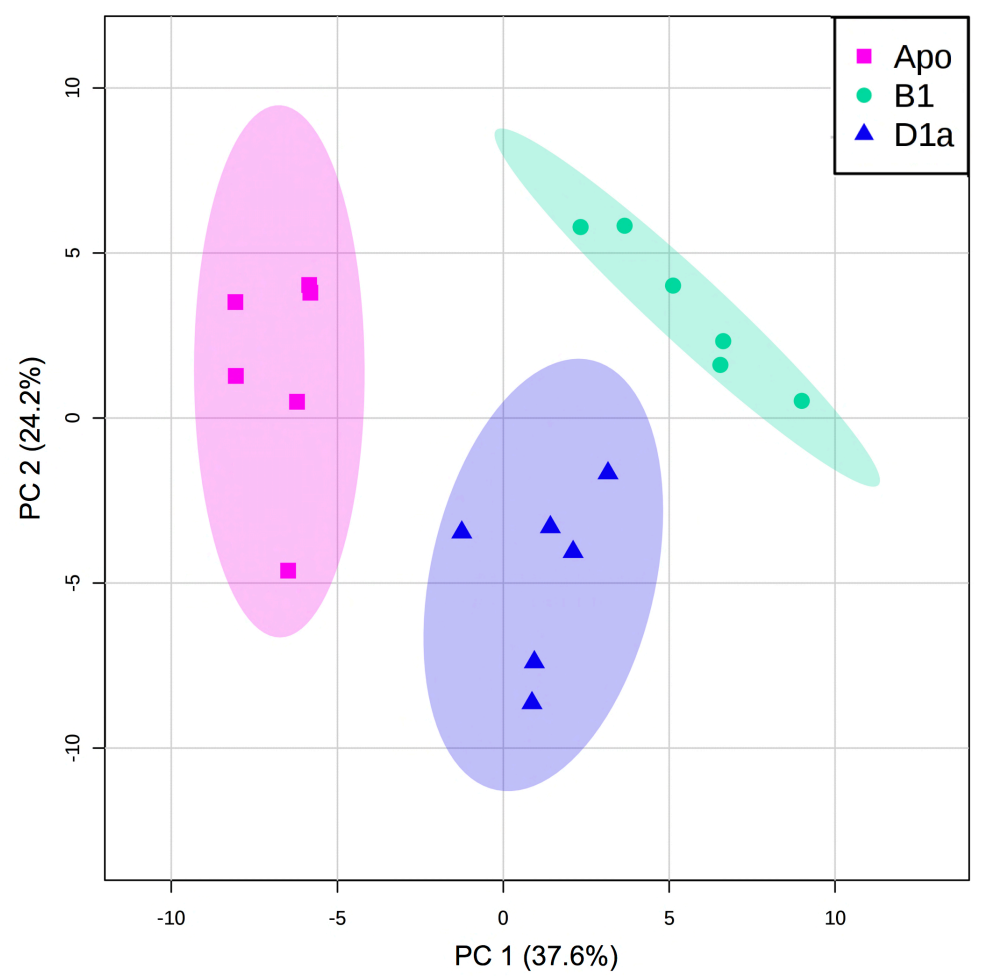

Figure 4.1 PCA scores plot of host metabolite profiles, with $95 \%$ confidence intervals. The relative abundance of the metabolites detected in host free pools between the three symbiotic states: aposymbiotic (Apo), homologous (B1), and heterologous (D1a) $(n=6)$. 


\subsubsection{Enrichment analysis of total host metabolite pools}

Non-targeted metabolite isotopomer enrichment analysis revealed that 31 of the 81 metabolites identified were labelled (Table S4.2). Aspartic acid was labelled in the aposymbiotic host and thus was removed from further analyses.

Comparative enrichment analysis revealed that nine metabolites were labelled in both homologous B1-colonised and heterologous D1a-colonised host tissues. These metabolites included sugars and sugar alcohols, amino acids and fatty acids (Table 4.2). The inositol isoform scyllo-inositol had the highest percentage label in the homologous B1-colonised host (33\% labelled), followed by glucose, mannitol and palmitoleic acid (23\% labelled each) (Table 4.2). In heterologous D1a-colonised host tissues, glucose and mannitol showed the highest percentage label (39\% labelled each) (Table 4.2). The greatest difference was found for scylloinositol, for which the labelled fraction of was 40-fold more abundant in B1- than D1acolonised host pools, and the unlabelled fraction was 11-fold more abundant (Table 4.2).

To investigate functional differences between symbioses, the total relative abundance of the labelled and unlabelled fractions of each enriched metabolite was compared between Symbiodinium types (Table S4.3; Figure 4.2). Both the unlabelled and labelled fractions (of the total relative abundance) of glucose, scyllo-inositol, mannitol, and myristic acid (C14:0) were significantly more abundant in B1- than D1a-colonised host pools (Table S4.3; Figure 4.2). The labelled fractions of glutamate and palmitoleic acid (C16:1) were also significantly more abundant in B1- than D1a-colonised host pools (Table S4.3; Figure 4.2). The total pools of glucose (Mann Whitney $U<0.001 ; n=12, p=0.002$ ), scyllo-inositol (Students $t$-Test; $t=$ 5.906, $\mathrm{df}=5.027, p=0.002$ ), mannitol (Students $t$-Test; $t=6.509$, $\mathrm{df}=5.108, p<0.001$ ), and myristic acid (Students $t$-Test; $t=11.154, \mathrm{df}=10, p<0.001$ ) were significantly more abundant in B1-colonised host tissues (Table S4.3; Figure 4.2). In contrast, none of the enriched metabolites found in both symbiotic states were significantly more abundant in the D1a- than B1- colonised anemones. 
Table 4.2. Fold difference and percent label of the mean net metabolite abundance for the nine commonly labelled metabolites. Font colour of the fold difference in relative abundance (B1/D1a) denotes fold larger (red) or fold lower (blue) in homologous B1-colonised host pools compared to heterologous Dla-colonised host pools. Bold font denotes significantly different relative abundances as described in Table S4.3 $(p<0.05)$.

\begin{tabular}{|c|c|c|c|}
\hline & Labelled pool & Unlabelled pool & $\%$ Labelled \\
\hline Metabolite & $\begin{array}{l}\text { Fold difference } \\
\text { (B1/D1a) }\end{array}$ & $\begin{array}{l}\text { Fold difference } \\
\text { (B1/D1a) }\end{array}$ & $\begin{array}{l}\text { Homologous (B1) } \\
\text { Heterologous (D1a) }\end{array}$ \\
\hline Arginine & 1.27 & 1.27 & 12 \\
\hline Glucose & 4.47 & 9.81 & \begin{tabular}{l|l|l}
23 & 39
\end{tabular} \\
\hline Glutamate & 2.37 & -1.11 & \begin{tabular}{l|l}
7 & 17
\end{tabular} \\
\hline scyllo-Inositol & 40.31 & 10.91 & $\begin{array}{lll}12 & 33\end{array}$ \\
\hline Leucine & 1.39 & 1.1 & 10 \\
\hline Mannitol & 5.05 & 10.69 & 23 \\
\hline Myristic acid C14:0 & 2.04 & 5.14 & \begin{tabular}{l|l}
8 & 18
\end{tabular} \\
\hline Palmitoleic acid C16:1 & 3.85 & -1.16 & $\begin{array}{ll}6 & 23\end{array}$ \\
\hline Stearic acid C18:0 & 1.06 & 1.46 & 13 \\
\hline
\end{tabular}



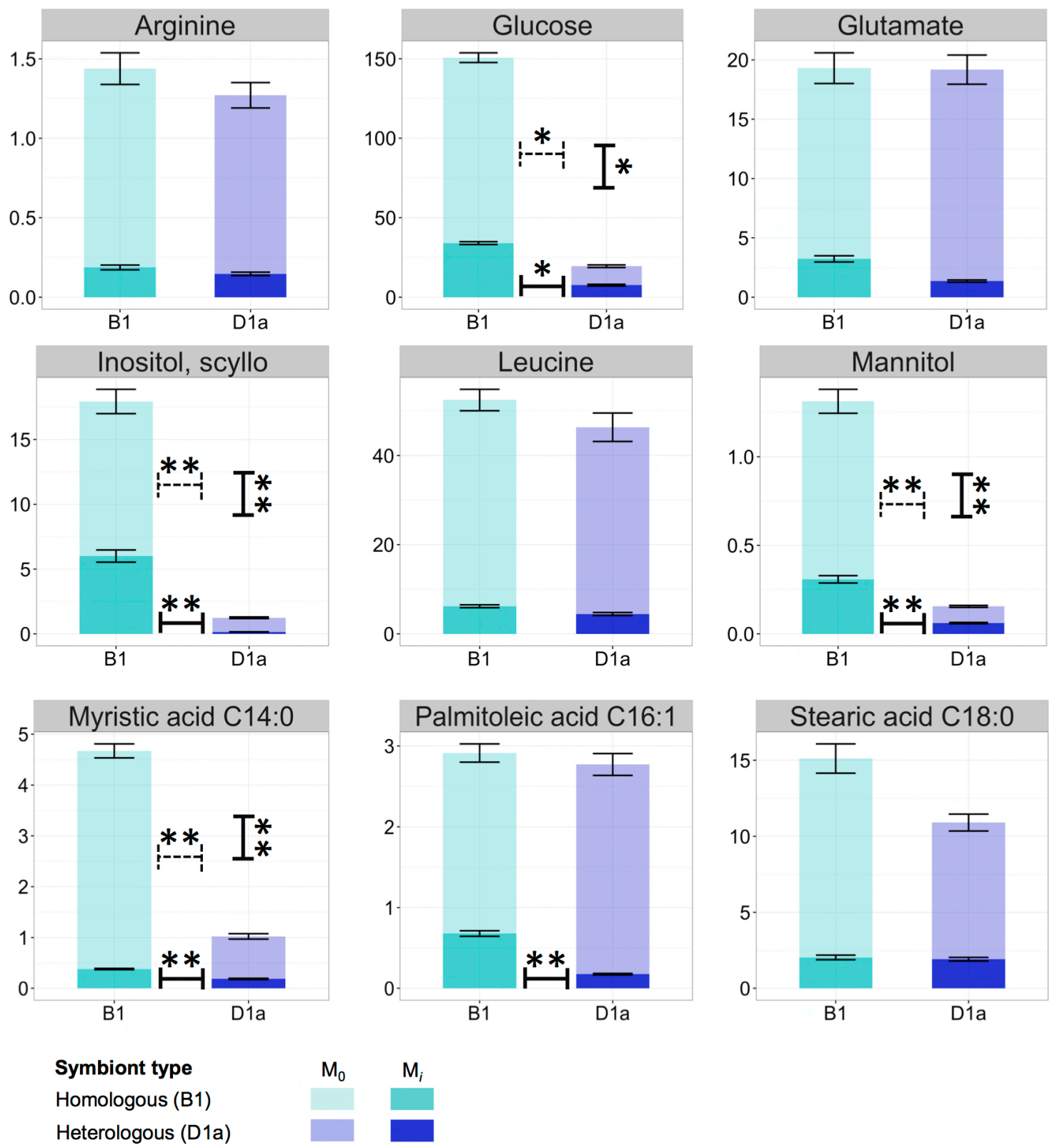

Figure 4.2 Mean net relative abundance and enrichment of metabolites found in both homologous and heterologous host tissues. Data are normalised relative mean abundances + S.E.M of the unlabelled $\left(\mathrm{M}_{0}\right)$ fraction and the labelled $\left(\mathrm{M}_{i}\right)$ fraction of the total pool. Dashed and solid horizontal brackets represent significant differences between the symbiont types in $\mathrm{M}_{0}$ and $\mathrm{M}_{i}$, respectively. Vertical brackets represent significant differences between the total pools. Asterisks indicate level of significance as described in Table S4.3; * $p<0.05$, ** $p<$ 0.001 .

\subsubsection{Enrichment analysis per symbiont cell}

Following normalisation to allow for differences in symbiont cell density, both the labelled and unlabelled fractions of scyllo-inositol, glucose and mannitol continued to be significantly more abundant in B1-colonised tissues (Table S4.4). The unlabelled fraction of myristic acid was significantly more abundant in B1-colonised tissues after normalisation, however the labelled 
fraction was not significant once normalised to symbiont cell density. The labelled fraction of palmitoleic acid (C18:1) continued to be significantly larger in the presence of B1, being up to 7-fold more abundant (Table S4.4). However, the labelled fraction of glutamate was not significant once normalised to symbiont cell density. Again, none of the labelled or unlabelled fractions of these compounds were significantly more abundant in the presence of D1a than B1.

\subsubsection{Symbiont specific metabolite translocation}

Non-targeted enrichment analysis revealed Symbiodinium type-specific labelled metabolites in the host pools. A total of 18 labelled metabolites were identified solely in homologous B1colonised host pools, with no label being detected in these same metabolites in the heterologous D1a-colonised host (Table 4.3).

In B1-colonised host pools, Symbiodinium type-specific labelling included the sugars fructose, sucrose, maltose and xylose. Sucrose and fructose showed especially high levels of enrichment (34\% and 22\%, respectively). The saturated fatty acid palmitic acid (C16:0), and unsaturated fatty acids oleic acid (C18:1n-9) and linoleic acid (C18:2n-6), and another isoform of inositol, myo-inositol, was also labelled in only B1-colonised host tissues.

Three unidentified metabolites were enriched in B1-colonised host tissues and are hence described as 'Unknown', followed by their relative retention times. The mass spectral information and the similarity of their MIDs to other labelled compounds in the dataset provided putative compound classifications (Weindl et al. 2016b): Unknown_19.53 was similar to fructose (Appendix Figure A1); Unknown_22.54 to stearic acid, Unknown_28.8 to maltose (Appendix Figure A2).

Conversely, three labelled metabolites were detected in the metabolite pools of D1a-colonised anemones that were not labelled in the homologous host tissues (Table 4.3). These consisted of tyramine, and the two amino acids glutamine and ornithine (Table 4.3). These metabolites showed low levels of labelling, at less than $7 \%$ each. 
Table 4.3. Enriched compounds found in either homologous (green) or heterologous (blue) host tissues. The mean net relative abundance of unlabelled $\left(\mathrm{M}_{0}\right)$ and labelled $\left(\mathrm{M}_{i}\right)$ fractions $( \pm$ S.E.M), and percentage labelled of the total ion abundance.

\begin{tabular}{|c|c|c|c|}
\hline Metabolite & $\mathbf{M}_{0}$ & $\mathbf{M}_{i}$ & $\%$ Labelled \\
\hline \multicolumn{4}{|c|}{ Homologous type B1-colonised host tissues only } \\
\hline Fructose & $2.59+0.85$ & $0.72+0.24$ & 22 \\
\hline Galactosylglycerol & $0.62+0.07$ & $0.07+0.01$ & 10 \\
\hline Gluconic acid & $0.86+0.18$ & $0.19+0.04$ & 18 \\
\hline Glyceric acid & $2.31+0.18$ & $0.27+0.02$ & 10 \\
\hline Guanosine & $0.70+0.12$ & $0.04+0.01$ & 6 \\
\hline Palmitic acid C16:0 & $36.92+4.17$ & $3.96+0.45$ & 10 \\
\hline Inositol, myo & $5.78+0.73$ & $0.46+0.06$ & 7 \\
\hline Maltose & $36.17+3.96$ & $5.17+0.57$ & 12 \\
\hline Linoleic acid C18:2 & $2.88+0.38$ & $0.18+0.02$ & 6 \\
\hline Oleic acid C18:1 & $11.48+1.13$ & $0.74+0.07$ & 6 \\
\hline Pyroglutamic acid & $26.27+4.46$ & $4.12+0.70$ & 14 \\
\hline Serine & $27.78+3.53$ & $1.39+0.18$ & 5 \\
\hline Sucrose & $8.58+0.91$ & $4.44+0.47$ & 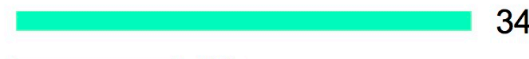 \\
\hline Threonic acid & $1.95+0.26$ & $0.27+0.04$ & 12 \\
\hline Unknown_22.54 & $0.73+0.14$ & $0.07+0.01$ & 9 \\
\hline Unknown_28.8 & $0.64+0.07$ & $0.11+0.01$ & 15 \\
\hline Unknown_19.53 & $0.37+0.06$ & $0.14+0.02$ & 27 \\
\hline Xylose & $6.83+1.32$ & $0.37 \pm 0.07$ & 5 \\
\hline \multicolumn{4}{|c|}{ Heterologous type D1a-colonised host tissues only } \\
\hline Glutamine & $0.04+0.02$ & $0.003+0.001$ & 6 \\
\hline Ornithine & $0.78+0.13$ & $0.06+0.01$ & 7 \\
\hline Tyramine & $11.14+3.63$ & $0.77+.025$ & 6 \\
\hline
\end{tabular}

\subsubsection{Pathway visualisation}

Metabolite identification, a priori knowledge of metabolic pathways, and pairwise similarity in MID of the isotopically-enriched metabolites, enabled analysis of their metabolic proximity and correlation of biosynthetic pathways with the associated Symbiodinium type (Weindl et al. 2016b) (Figure 4.3). In the B1-colonised host, labelling occurred in a variety of processes involved in central metabolism (glycolysis and pentose phosphate pathways), and glycerolipid and fatty acid synthesis. In contrast, in the D1a-colonised host, labelling was primarily associated with the urea cycle and fatty acid synthesis (Figure 4.3). 


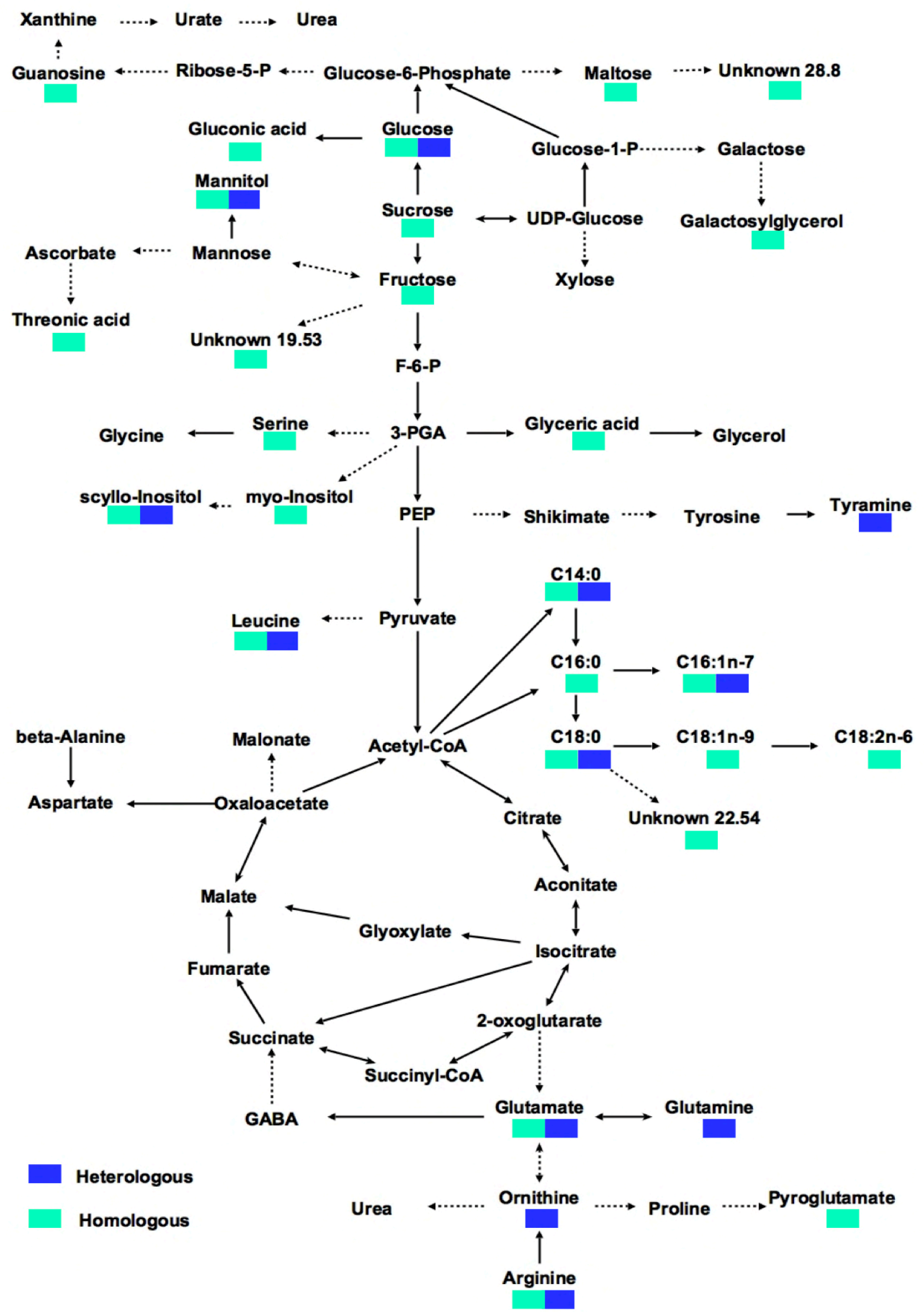

Figure 4.3 Summary schematic of the enriched compounds detected in the host free pools in homologous type-B1 and heterologous type-D1a associations. Green or blue squares denote isotopic labelling was detected in the homologous B1-colonised or heterologous D1acolonised host tissues, respectively. For simplicity, not all labelled metabolites are shown. 


\subsection{Discussion}

Although the diversity of Symbiodinium has the potential to provide the host with increased functional range and resilience to physical stressors such as elevated temperature, many corals form specific associations with distinct Symbiodinium species. One key consideration in the hosts' selection of compatible algal symbionts is the role played by nutritional exchange. How symbiont type affects the identity and quantity of translocated carbon compounds and the downstream pathways, and how the patterns of translocation relate to symbiosis specificity remain unclear. My results demonstrate the nutritional implications of hosting a novel symbiont type (Symbiodinium D1a) in the cnidarian-dinoflagellate symbiosis. While there is some similarity in the identity and quantity of translocated metabolites when hosting homologous Symbiodinium B1 and heterologous Symbiodinium D1a, there are notable qualitative and quantitative differences with respect to both the translocated metabolites and their consequences for downstream metabolite pools. Specifically, the diversity and abundance of many enriched metabolites were lower in the presence of D1a. Given that metabolic exchange is a central feature of the symbiosis, such differences may have consequences for the persistence of novel host-symbiont combinations, and the adaptive capacity of reef corals to climate change.

\subsubsection{Colonisation dynamics}

Homologous Symbiodinium B1 reached higher cell densities than did heterologous Symbiodinium D1a; this is consistent with previous studies of clade D colonisation in Aiptasia (Leal et al. 2015). Similarly, most colonisation studies have shown that homologous symbiont types are more readily accepted by their hosts, and that heterologous types are either rejected or suffer lower population growth rates if they are accepted (Kinzie 1974; Kinzie and Chee 1979; Schoenberg and Trench 1980a; Trench 1987; Davy et al. 1997b; Trench 1997; Weis et al. 2001; Belda-Baillie et al. 2002; Rodriguez-Lanetty et al. 2003; Starzak et al. 2014). This is likely because of the underlying cellular processes involved symbiosis establishment, sorting and proliferation, though the exact nature of these processes remains unclear (Davy et al. 2012). Nevertheless, at the time of sampling, the results show that D1a-colonised Aiptasia had a similar autotrophic potential and symbiosis functionality to that seen in the presence of B1. This highlights the potential impact of symbiont density on photosynthetic activity in B1colonised hosts, with carbon limitation and hence reductions in photosynthetic rate per symbiont cell possibly occurring at the higher symbiont densities, irrespective of symbiont type. While the more minor differences in the observed enriched metabolite profiles may result 
from differences in the symbiont density at the time of sampling (B1 densities were 35.1\% higher than D1a), any significant differences in abundance $>35 \%$ may be considered a direct result of the symbiont type and not symbiont density effects. All statistically significant metabolites had a fold change $>2$ (i.e. $>100 \%$ difference in abundance). For example, I observed significantly large fold increases in the enrichment of glucose (4.5-fold), scylloinositol (40-fold), and mannitol (5-fold) in the presence of B1, and the various differences in the identity of the enriched compounds between the homologous vs. heterologous symbiotic states, strongly suggest that these differences are a direct result of the symbiont type.

\subsubsection{Symbiont type-specific modifications to glucose availability and lipogenesis}

My results corroborate evidence that glucose is a major translocated photosynthetic product in the cnidarian-dinoflagellate symbiosis (Whitehead and Douglas 2003; Burriesci et al. 2012; Hillyer 2016; Hillyer et al. 2016a). Both symbiont types seemingly translocated glucose, reiterating the importance of this metabolite to cellular homeostasis and ultimately the survival of the holobiont (Burriesci et al. 2012). Given the similar autotrophic potential, it would be expected that the rates of carbon fixation and energy production would be similar, and thus enriched glucose pools would exhibit similar relative abundances. Conversely, a decline in the abundance of labelled glucose suggests either a reduction in the production of glucose de novo, or less translocated glucose. Here, the relative abundance of the enriched glucose pools was 4.5-fold larger in B1-colonised hosts, and this difference remained when data were normalised to symbiont cell density. This suggests greater glucose synthesis de novo and translocation of the enriched compound in the homologous B1-colonised hosts.

Furthermore, the unlabelled pools of glucose were 10-fold larger in the B1-colonised hosts. Unlabelled metabolite pools have been derived from something other than symbiont autotrophy or downstream metabolism of translocated labelled compounds, such as host gluconeogenesis, or pools are turning over slowly and/or are very large and not yet enriched. In this regard, the total glucose pools were 8-fold more abundant in B1-colonised hosts, and thus the large pools were likely due to slow turnover and the accumulation of glucose; this likely allows for the storage of excess glucose as glycogen deposits in the B1-colonised hosts, so providing a nutritional benefit (Kopp et al. 2015). However the methods used here were unable to detect glycogen pool enrichment, and so this warrants further investigation.

Meanwhile, the notable smaller glucose pools in heterologous D1a-colonised host tissues suggest either (1) reduced glucose production de novo; (2) reduced translocation from the symbiont; (3) increased turnover of glucose metabolism; or (4) a combination of the above. 
Given the central role of glucose in energy metabolism, reduced availability of glucose when in symbiosis with D1a would thus necessitate the use of stored energy by the host (e.g., via gluconeogenesis) or increased heterotrophy to support its respiratory and metabolic requirements. Furthermore, glucose is an important source of organic carbon and is a precursor for many biomolecules including lipids and vitamins $\mathrm{C}$, thus reduced availability of glucose has potential longer-term implications for host growth and survival. These data support the growth costs attributed with hosting Symbiodinium members of clade D under normal conditions (Cantin et al. 2009; Jones and Berkelmans 2010; Cunning et al. 2015a), as well as the requirement for an increased heterotrophic contribution when harbouring a heterologous symbiont (Loram et al. 2007; Starzak et al. 2014; Leal et al. 2015). A similar mechanism is observed during dysbiosis as a result of coral bleaching, as the coral host metabolism may become more reliant on heterotrophically-derived carbon and the mobilisation of limited stored energy reserves to support its energetic needs (Loram et al. 2007; Hughes et al. 2010; Hughes and Grottoli 2013; Baumann et al. 2014; Leal et al. 2015; Hillyer et al. 2016a). Thus, the data not only the importance of trophic plasticity for the resilience of novel associations, but also suggest that once host energy stores are exhausted, inadequate nutritional exchange is likely to be an influencing factor in the maintenance of a novel symbiosis with D1a.

Further to the translocation of central metabolites such as glucose, the saturated fatty acids myristic acid (C14:0) and stearic acid (C18:0) were also enriched in both the symbiotic associations, with both labelled and unlabelled pools of myristic acid being significantly more abundant in the presence of B1. In comparison, pools of both labelled and unlabelled stearic acid were similarly abundant in the two symbiotic associations. However, in the presence of B1 alone, enrichment was detected in the products of stearic acid elongation (C18:1n-9 and C18:2n-6). C14:0, C18:0, C18:1n-9 and C18:2n-6 are all fatty acid and lipogenesis precursors, and similarly, the lipogenesis intermediate galactosylglycerol was only enriched in homologous B1-colonised host pools. The enhanced presence of lipogenesis intermediates suggests that de novo fatty acid and triglyceride lipid synthesis pathways are more active in the host and/or symbiont when in the homologous symbiotic state. More specifically, the observed enrichment of fatty acids in the homologous state is, presumably, a result of translocation of these metabolites, their precursors, or their lipogenesis products, as opposed to the degradation of enriched lipid stores. Meanwhile, the absence of labelled lipogenesis intermediates in the D1a-colonised hosts suggests that the enriched fatty acids were immediately utilised in central metabolic functions. This concurs with evidence that symbiont type has been shown to affect the abundance of stored lipids in corals (Cooper et al. 2011a). 
Taken together, the host exhibited increased fatty acid and lipid biosynthesis when infected with the homologous Symbiodinium B1, but little evidence for this when infected with Symbiodinium D1a. These data further supports the metabolic costs associated with hosting clade D symbionts under normal conditions found in previous studies (Cantin et al. 2009; Jones and Berkelmans 2011; Stat and Gates 2011; Jones and Berkelmans 2012; PErnice et al. 2015; Cunning et al. 2015a). For instance, juvenile corals harbouring members of clade D received more carbon than conspecifics hosting clade C (Cantin et al. 2009), and cnidarians hosting clade D exhibit slower growth rates (Little et al. 2004; Jones and Berkelmans 2010; Cunning et al. 2015a). Symbiotic associations with lower autotrophic contributions can compensate for such losses with increased feeding and digestion (Grottoli et al. 2006; FerrierPagès et al. 2011; Hughes and Grottoli 2013), and this has recently been shown in Aiptasia sp. when a heterologous association with clade D symbionts (Leal et al. 2015). Ultimately, this will have important consequences for the potential survival of this, and perhaps other, host species when in symbiosis with this heterologous Symbiodinium type.

\subsubsection{Symbiont type effects on host fatty acid and lipid composition}

Corals predominantly receive unsaturated fatty acids from their algal symbionts (Papina et al. 2003). My results show that the mono-unsaturated fatty acid palmitoleic acid (C16:1n-7) was enriched in both the homologous and heterologous symbiotic states, although a greater amount of enrichment was detected in the presence of B1. The enrichment of palmitoleic acid in both symbioses, despite the precursor palmitic acid (C16:0) only being enriched in the presence of B1, suggests that palmitoleic acid is likely a form in which saturated fatty acids are translocated from symbiont to host, independent of the symbiotic types tested here. Furthermore, C18:2n-6 was enriched in the homologous B1-colonised host pools only. As cnidarian hosts do not possess the $\Delta^{12}$ desaturase enzymes required to synthesise this metabolite from oleic acid C18:1n-9 (Dunn et al. 2012), it seems reasonable to assume that C18:2n-6 is translocated by the symbiont, highlighting a key difference between the physiology of Symbiodinium B1 and D1a. Of significance, C18:2n-6 is a precursor molecule to essential fatty acids; of which eicosapentaenoic acid (C20:5n-3), docosahexaenoic acid (C22:6n-3), and arachidonic acid (C20:4n-6) are the most important (Teece et al. 2011; Urich 2013). Similarly, C16:1n-7 is necessary for the synthesis of C16 polyunsaturated fatty acids in corals, and C16:0 and C16:1n-7 represent two of the main components of total lipids in the cnidarian-dinoflagellate consortium (Bishop and Kenrick 1980; Harland et al. 1991; A1Moghrabi et al. 1996; Zhukova and Titlyanov 2006; Kneeland et al. 2013). While corals are able to acquire essential fatty acids from the symbiont (Papina et al. 2003; Teece et al. 2011; 
Imbs et al. 2014) or through heterotrophic consumption, a change in fatty acid and lipid composition has been shown to reflect changes in nutrition, ecology and ultimately the health of corals (Oku et al. 2003b; Saunders et al. 2006), and thus is likely to influence the fate of a novel symbiosis.

\subsubsection{Symbiont type-specific impacts on the host oxidative environment}

Both partners of the coral-dinoflagellate symbiosis express a diverse repertoire of nonenzymatic and enzymatic antioxidants to prevent oxidative damage (Richier et al. 2005; Richier et al. 2006; Merle et al. 2007; Ganot et al. 2011; Krueger et al. 2015). Multiple antioxidant metabolites and precursors were labelled in both the homologous and heterologous symbiotic states, including mannitol and glutamate, consistent with the production of antioxidants by Symbiodinium, and the expression of a larger isoform diversity and activity of antioxidants when in symbiosis (Dykens and Shick 1982; Richier et al. 2005). However, the abundance and diversity of these antioxidants differed between the two symbiotic states.

Modulation of the oxidative environment and redox-related signal transduction have been suggested to feature prominently in the evolution of novel mutualistic symbioses (Moné et al. 2014). For example, reactive oxygen species (ROS) regulation plays an important role in the tolerance of resident bacteria and elimination of pathogenic ones in Drosophila melanogaster (Ha et al. 2009). Similar oxidative regulatory mechanisms may therefore occur in the cnidarian-dinoflagellate symbiosis; indeed, besides the role of ROS and reactive nitrogen species (RNS) accumulation in the bleaching cascade of the cnidarian-dinoflagellate symbiosis (Weis 2008; Lesser 2011), successful infection of Aiptasia with homologous symbionts results in a downregulation of host nitric oxide production pathways (Detournay et al. 2012), while infection of Aiptasia with unsuitable or dysfunctional symbiont cells may lead to heightened host nitric oxide synthesis (Hawkins 2013) and evidence of host oxidative stress (Chapter 3).

Mannitol was enriched in both associations, but homologous B1-colonised hosts showed a 5fold and 10-fold increase in the abundance of labelled and unlabelled pools, respectively. Mannitol acts as a compatible solute in algae, with its functions including osmoregulation, sugar storage, and non-enzymatic scavenging of hydroxyl radicals (Lesser 1997). Many plant pathogens have the ability to synthesise mannitol, and there is growing evidence that phytopathogenic fungi use mannitol to suppress ROS mediated defences in the host (Jennings et al. 1998). Therefore mannitol translocation may provide a mechanism for avoiding host defences via redox homeostasis in the establishment and maintenance of the cnidariandinoflagellate symbiosis (Voolstra et al. 2009; Schnitzler and Weis 2010). In this regard, the 
lower abundance of mannitol in Dla-colonised hosts may therefore indicated increase turnover in order to maintain oxidative homeostasis. Of note though, mannitol is also known to be a primary product and storage form of sugar derived from photosynthesis in algae (Bidwell 1967; Loescher et al. 1992; Obata et al. 2013). Although the role of mannitol translocation in the cnidarian-dinoflagellate symbiosis is unclear, more successful colonisations of Symbiodinium may involve increased mannitol translocation; this warrants further investigation.

Further to the translocation of antioxidant metabolites and precursors, was evidence of labelling in glutamate in both associations. Glutamate and glutamine are precursors for the antioxidant glutathione (Yellowlees et al. 2008; Meyer and Weis 2012), and supply of the precursors may serve to maintain high levels of glutathione and avoid oxidative damage (Amores-Sánchez and Medina 1999). Enriched glutamate was 2.4-fold more abundant in B1colonised hosts, however pools were not significantly larger after normalisation to symbiont density and the unlabelled pool abundance was similar in both the homologous and heterologous symbiotic states before and after normalisation to symbiont density. Meanwhile, only D1a-colonised hosts exhibited enrichment of glutamine pools, however the percentage labelled of the total abundance was low (7\%) and total pools of glutamine were 7.5-fold larger in the B1-colonised hosts. This indicates either high turnover and/or downstream metabolism of these precursors and potentially increased glutathione metabolism in D1a-colonised hosts, perhaps caused by an imbalance in the oxidative environment in the heterologous host.

Furthermore, my analysis revealed enrichment of the shikimate bi-product tyramine in D1acolonised anemones only. The shikimate pathway produces mycosporine-like amino acids (MAAs), which act as non-enzymatic antioxidants, with functional roles as ROS scavengers and UV-absorbing compounds (Banaszak and Trench 1995; Dunlap and Yamamoto 1995; Kim et al. 2001; Shick and Dunlap 2002; Rosic and Dove 2011). The shikimate pathway has been identified in both symbiotic cnidarians and their dinoflagellate partners, and ROS stress has been shown to increase biosynthesis of MAA compounds (Rosic and Dove 2011). Moreover, MAA compounds have been suggested to be translocated between the partners (Shick 2004; Rosic and Dove 2011). Enrichment in shikimate bi-products may therefore indicate antioxidant-provisioning by D1a, or increased shikimate metabolism using labelled compounds when anemones host D1a, providing mechanisms for modulating the oxidative environment.

Collectively, the translocation and increased turnover of mannitol, high turnover and downstream metabolism of glutathione precursors, and D1a-specific MAA enrichment, support evidence that a heterologous association causes a disruption in host cell oxidative homeostasis 
(Chapter 3). The translocation of antioxidant metabolites is a potential mechanism of thermoand oxidative-tolerance afforded to the holobiont by the Symbiodinium cells (Krueger et al. 2014), and different genotypes may provide different amounts or varieties of antioxidants (Klueter et al. 2015). In this regard, a greater thermo- and oxidative-tolerance is attributed to Symbiodinium type D1a (Berkelmans and Van Oppen 2006; Stat and Gates 2011), and may be linked to the photoprotective and antioxidant compound repertoire of clade D (McGinty et al. 2012; Wietheger 2012). Therefore, the antioxidant response of type D1a may explain its ability to form the functional novel association presented here.

\subsubsection{Evidence for the increased activity of the urea cycle in the heterologous association}

Glutamate and glutamine also play important roles in the urea cycle, along with ornithine and arginine. Arginine was labelled here in both the homologous and heterologous associations, however the D1a-colonised anemones also exhibited enrichment in ornithine and glutamine pools while B1-colonised anemones did not. Ammonium is assimilated either by the dinoflagellate via the glutamine synthase/glutamine:2-oxoglutare aminotransferase (GS/GOGAT) cycle (Roberts et al. 1999; Roberts et al. 2001; Pernice et al. 2012), or by the cnidarian host through the action of glutamine synthase and glutamate dehydrogenase (Wang and Douglas 1998; Yellowlees et al. 2008). Therefore the abundance of both glutamate and glutamine are supposed to be a sensitive indicator of nitrogen assimilation following uptake of ammonium by the dinoflagellate (Flynn et al. 1994). When considered in a pathway context, these data suggest that the labelled compounds were being utilised in the urea cycle in the D1acolonised host. This is of particular interest given that nitrogen cycling within the symbiosis includes ammonium produced via the urea cycle (Wang and Douglas 1998; Grover et al. 2006; Yellowlees et al. 2008). It is possible that translocated compounds were utilised in the urea cycle in the heterologous association in order to support symbiont photosynthesis, and therefore allow for the persistence of a functional symbiosis.

Similarly, increases in the urea cycle have been proposed as a mechanism to promote symbiont replication (Rees 1989). Therefore, the urea cycle activity here may potentially encourage the proliferation of heterologous D1a symbionts. Further to this, there is evidence that control of nitrogen provision (particularly excretory ammonium) to the symbiont is important for the regulation of host-symbiont biomass e.g. (Smith and Muscatine 1986; Muscatine et al. 1989b; McAuley and Cook 1994; Davy et al. 2012). Therefore, as Symbiodinium cell densities were larger in B1-colonised anemones, the absence of enrichment in ornithine and glutamine in this 
symbiosis suggests a reduction in ammonium producing pathways, which could act to limit symbiont growth and biomass, and prevent overgrowth of host tissues.

\subsubsection{Inositol and maltose signalling for a successful symbiosis}

Interestingly, scyllo-inositol was enriched in host pools in both homologous and heterologous associations, however the abundance of both the labelled and unlabelled pools was substantially larger in the B1-colonised anemones. Indeed, the labelled pool was 40-fold more abundant when B1 was present. Similarly, the unlabelled fraction was 10-fold more abundant when anemones were colonised by B1. Inositol signalling and metabolism have recently been described as common pathways in Symbiodinium (Rosic et al. 2015), while different Symbiodinium cultures exhibit different expression patterns of inositol isoforms in response to temperature and light treatments (Klueter et al. 2015). Moreover, multiple putative sugar/inositol co-transporters have been identified in cnidarian transcriptomes, and were upregulated in symbiotic versus aposymbiotic anemones (Lehnert et al. 2014; Baumgarten et al. 2015). Inositol and inositol phosphate signalling feature prominently in plant signal transduction (Gillaspy 2011), perhaps indicating that they play a comparable role in the symbiosis, particularly if the transporters are located on the symbiosome (Lehnert et al. 2014). Although the exact role of inositol signalling in the symbiosis is unclear, increasing evidence suggests that inositol compounds are likely to participate in the symbiosis signalling, specificity and dysfunction (Dunn et al. 2004; Dunn et al. 2007; Weis 2008; Hillyer 2016);

\section{Chapter 3).}

Also of interest with respect to inter-partner signalling, both maltose and an unidentified product of maltose metabolism were labelled in B1-colonised hosts only, and total pools of maltose were 46-fold larger in B1-colonised host tissues; this is consistent with the findings of Hillyer et al. (2016), who found maltose in the host fraction of both Aiptasia and the coral Acropora aspera. Maltose is the chief photosynthetically-derived metabolite translocated in the Chlorella-Hydra symbiosis, where it plays a role in microbe assisted molecular patterning (MAMP) recognition of the algal symbionts (Hohman et al. 1982; McAuley and Smith 1982a). It is therefore conceivable that maltose also plays a role in the establishment and maintenance of the cnidarian-dinoflagellate symbiosis, though this requires further investigation. 


\subsection{Conclusion: Metabolic flux and the fate of the symbiosis}

Both partners can influence the fate of a novel association, from the host side by the intensity of the immune response, and from the symbiont side by the degree of biological perturbations inflicted on the host (Moné et al. 2014). Taxonomic variation in Symbiodinium metabolite profiles exists (Klueter et al. 2015), and given that nutrient cycling is central to the symbiosis, may contribute to the observed host-symbiont specificity, although the mechanisms of symbiosis-specificity are likely to be more complex, particularly in regards to the physiological, ecological, biogeographical diversity of Symbiodinium (Baker 2003). Nevertheless, my results show that the identity and quantity of photosynthate translocated from the symbiont differs when Aiptasia harbours different Symbiodinium types, and that this can cause differences in the downstream metabolite profile of the host (Figure 4.4). In turn, unless the symbiosis is able to adapt to these differences (e.g. via heterotrophic compensation (Grottoli et al. 2006; Hughes and Grottoli 2013; Leal et al. 2015)), this will likely affect the physiology of the symbiosis and potentially its long-term chance of survival.

Of particular note, pathway activity in B1-colonised hosts was consistent with lipogenesis and glycolysis, the regulation of host-symbiont biomass and/or molecular signalling, while D1acolonised hosts had a lower diversity of labelled metabolites, with an emphasis on urea synthesis. Further investigation, utilising more Symbiodinium genotypes spanning a variety of physiological traits, and preferably attaining similar cell densities will help to shed further light on these mechanisms, as will the identification of thus far unidentified metabolites. Moreover, studies investigating the role of antioxidants in symbiosis establishment and maintenance, and in symbiosis-specificity, should be a priority for future research. The application of nontargeted stable isotope metabolomics approaches as applied here will provide a powerful means of further understanding the role played by nutritional exchange in the formation of novel symbiotic associations, and the capacity of reef corals to adapt to climate change. 


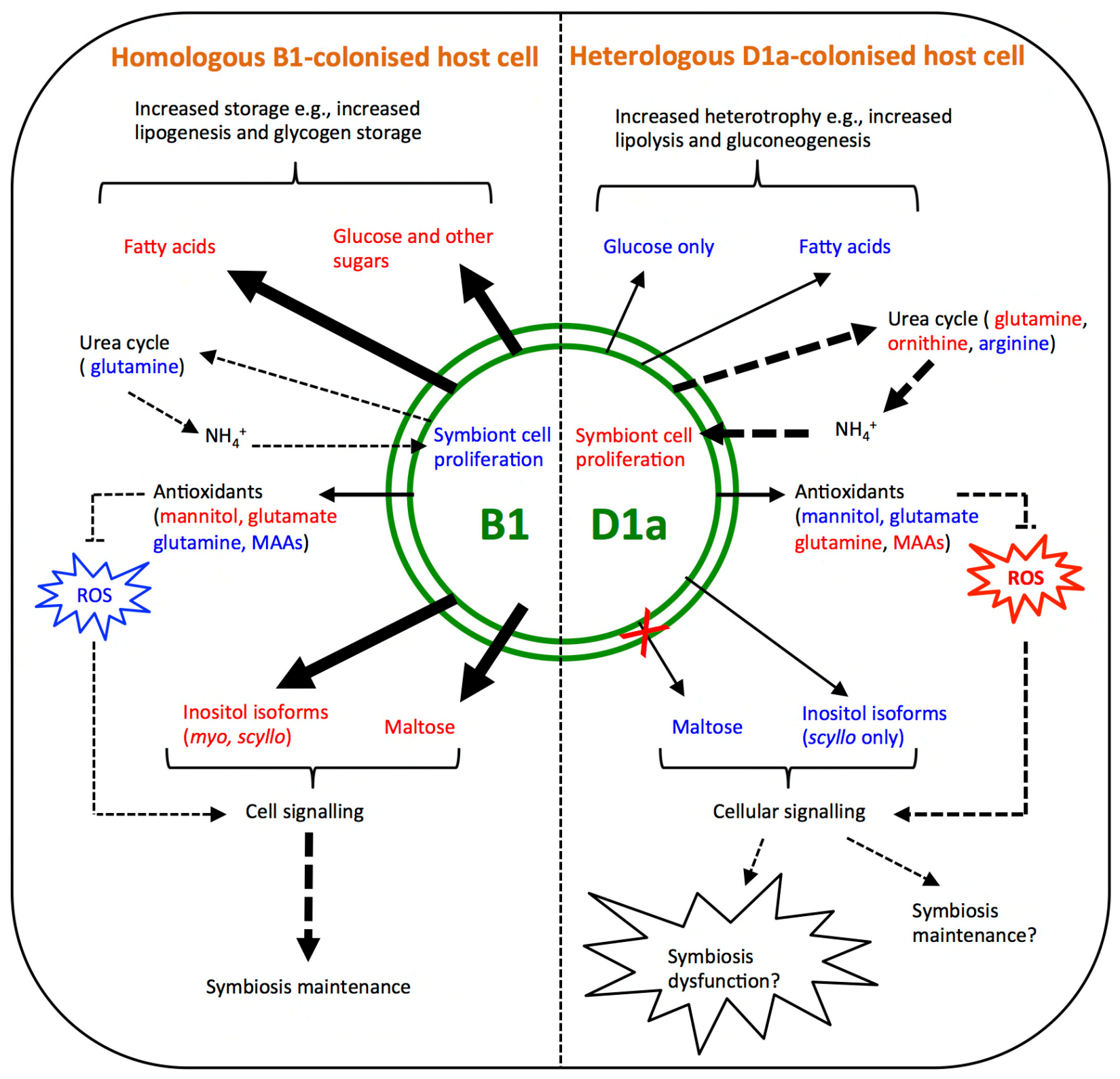

Figure 4.4 Summary diagram of the metabolic flux and cellular consequences of harbouring different Symbiodinium types. The metabolites identified as enriched in the homologous B1-colonised host cell (left) and heterologous D1a-colonised host cell (right) indicate the downstream metabolic response of the host cell and how this might affect the fate of the symbiosis. Font colour indicates larger (red) or smaller (blue) relative abundance. Arrow thickness indicates activity, with thick arrows indicating a very active pathway and thinner arrows relatively less activity. Dashed arrows are putative responses. 


\section{CHAPTER 5}

\section{Metabolite pools of the reef building coral Montipora capitata are unaffected by Symbiodinium community composition}

\subsection{Introduction}

Photosynthetic symbioses are prevalent in the marine environment, allowing the animals to thrive in oligotrophic environments (Venn et al. 2008). The association between dinoflagellates of the genus Symbiodinium and coral reef cnidarians represents a key photosynthetic symbiosis in oceans (Freudenthal 1962; Trench 1987). Within cnidarian tissues, Symbiodinium are typically located within the symbiosome membrane complex in host gastrodermal cells (Roth et al. 1988; Wakefield and Kempf 2001). The cnidarian host capitalise on the organic products of photosynthesis from the symbiont, including glycerol, alanine, fatty acids and lipids, although most recent studies point to glucose as being the major translocated photosynthetic product in the symbiosis (Whitehead and Douglas 2003; Burriesci et al. 2012; Hillyer et al. 2016a; Chapter 4). These metabolites contribute significantly to a coral's energetic budget as well as promoting deposition of the calcium carbonate skeleton (Muscatine and Cernichiari 1969; Muscatine 1973; Muscatine and Porter 1977; Wang and Douglas 1999; Burriesci et al. 2012; Kopp et al. 2015). In return, the host provides access to dissolved inorganic nutrients (carbon, nitrogen and phosphorus) and may also exchange host-derived amino acids, lipids and fatty acids (Wang and Douglas 1999; Imbs et al. 2014). This reciprocal exchange is central to the ecological and evolutionary success of coral reefs (Muscatine and Porter 1977). However, environmental stress, such as increased seawater temperature, can affect the stability of this relationship when photosynthesis is disrupted, compromising the mutually beneficial state and ultimately resulting in the breakdown of the symbiosis (known as coral bleaching) (Weis 2008). This loss of a functional symbiosis is reversible, but can result in mortality when prolonged or severe (Hoegh-Guldberg 1999; McClanahan 2004; Montano et al. 2010). Owing to the large scale at which heat-induced coral bleaching and mortality can occur, it is considered a major extinction threat for many reef coral species (Hoegh-Guldberg et al. 2007; Carpenter et al. 2008).

A coral species can adapt to a new environmental regime (i.e. light, temperature, depth) by associating with different Symbiodinium types (Iglesias-Prieto et al. 2004; Sampayo et al. 
2007; Frade et al. 2008). This is because of the considerable molecular, ecological, and physiological diversity within the genus Symbiodinium, with nine clades (A-I) and numerous sub-clades ('types') known to exist (Pochon and Gates 2010). Physiological diversity has been shown at the clade and type levels with respect to a range of functional characteristics, including stress tolerance levels (Buddermeier and Fautin 1993; Loram et al. 2007; Sampayo et al. 2008b). As a result, different symbiont types confer different phenotypes to the coral host, including thermal resistance to prevent coral bleaching (Little et al. 2004; Mieog et al. 2009; Jones and Berkelmans 2011). For instance, corals harbouring symbionts from clade D typically demonstrate higher thermotolerances than conspecifics hosting clade $\mathrm{C}$ members (Glynn et al. 2001; Berkelmans and Van Oppen 2006; Sampayo et al. 2008b; Stat and Gates 2011; Silverstein et al. 2015).

Although flexibility in the Symbiodinium community composition may allow for the maintenance of symbiotic function and holobiont stability following environmental disturbance (Jones et al. 2008; Silverstein et al. 2015; Boulotte et al. 2016), these changes are mostly temporary and a dominant type tends to persist in the long term, indicating long-term fidelity and stability in these associations (Thornhill et al. 2006; Sampayo et al. 2008a; Coffroth et al. 2010; Sampayo et al. 2016). For example, in a 32-month reciprocal depth transplantation experiment, Seriatopora hystrix colonies maintained a dominant association with type C3nt in response to prolonged environmental change, and Stylophora pistillata and Pocillopora damicornis colonies underwent temporary changes but these changes were restricted to 'hostcompatible' symbionts and reversion to original symbionts occurred within 7-12 months (Sampayo et al. 2016). These patterns may be a consequence of the functional attributes of specific symbionts; for example, as nutritional exchange is fundamentally important to the symbiosis, these patterns may be associated with the quantity and identity of nutrients translocated to the host by different symbiont types. In support of this hypothesis, different Symbiodinium types exhibit different metabolite profiles when in culture (Klueter et al. 2015), and symbiont genotypic diversity affects the quantity and identity of material translocated to the host (Trench 1971a; Miralles et al. 1989; Loram et al. 2007; Cantin et al. 2009; Starzak et al. 2014; Leal et al. 2015; Chapter 4), ultimately impacting host growth rate (Little et al. 2004; Jones and Berkelmans 2011; Cunning et al. 2015a). More specifically, under optimal environmental conditions for growth, clade $\mathrm{C}$ symbionts show greater photosynthate contribution to the cnidarian host than do clade D symbionts (Cantin et al. 2009), and higher growth rates were observed for Pocillopora damicornis when dominated by clade $\mathrm{C}$ than when dominated by clade D (Cunning et al. 2015a). Furthermore, recent utilisation of nanoscale 
secondary ion mass spectrometry (nanoSIMS) has shown that Symbiodinium types from clades $\mathrm{C}$ and $\mathrm{D}$ that form natural associations with the coral Isopora palifera possessed different metabolic capabilities; specifically, the $\mathrm{C}$ type symbionts fixed and passed significantly more carbon and nitrogen to the coral host than the D type symbionts (Pernice et al. 2015). As a result, it is unclear whether, in the long term, nutritional costs might outweigh the ecological benefits of changes in Symbiodinium community composition for reef survival (Ortiz et al. 2013; Cunning et al. 2015a).

Despite the potential impacts on host nutrition under 'normal' conditions, some corals (e.g. Pocillopora damicornis and Montipora capitata) that host multiple symbiont types can form stable, dominant associations with clade D Symbiodinium (McGinley et al. 2012; Cunning et al. 2016). One reason for this may relate to changes in the host's feeding behaviour, as corals that receive a lower autotrophic contribution from their symbionts can compensate for this with increased heterotrophic feeding and digestion (Grottoli et al. 2006; Ferrier-Pagès et al. 2011; Hughes and Grottoli 2013); this has also recently been shown in the sea anemone Aiptasia sp. when in association with clade D Symbiodinium (Leal et al. 2015). The potential may therefore exist for corals to successfully adjust their dominant symbiont type and survive in the face of climate change, though we still need to know more about the physiological implications of shifting the dominant symbiont type. In particular, we know little about the effect of Symbiodinium community composition on the identity and quantity of metabolite pools in the host, and how this might impact upon the functional capacity of a holobiont to persist.

Metabolite profiling with the use of gas chromatography (GC) coupled with mass spectrometry (MS) is a powerful tool for accurately identifying and quantifying metabolites in a biological sample, and has contributed to our understanding of the complex molecular interactions in biological systems, including symbioses such as the Medicago truncatula-mycorrhizal symbiosis (Bino et al. 2004; Schliemann et al. 2008; Rasmussen et al. 2009). The high sensitivity of MS coupled with its ability to detect and quantify a wide range of metabolites from complex mixtures (i.e. cellular extracts) makes it well suited for the analysis of the major and minor compounds found in cnidarian and algal cells (Schauer et al. 2005; Viant 2007; Gordon and Leggat 2010; Davy et al. 2012; Lehnert et al. 2014; Hillyer et al. 2016a; Hillyer et al. 2016b; Sogin et al. 2016).

The focus of this study was to compare the intracellular free metabolite profiles of both the coral host and dinoflagellate symbiont with respect to natural variations in the Symbiodinium community composition. I conducted an in situ field survey in Kaneohe Bay, Hawai'i, of the 
metabolite profiles of Montipora capitata, a dominant reef-building coral that forms a symbiosis with Symbiodinium in clades C and D (LaJeunesse and Thornhill 2011; Stat et al. 2013; Cunning et al. 2016). Direct field sampling was conducted to avoid any changes in symbiont community composition and to capture the metabolite profile under field conditions, and supporting environmental data and photophysiological measures were collected to aid data interpretation. The results show that both host and symbiont metabolite profiles were unaffected by Symbiodinium community composition, despite previous studies leading me to expect otherwise. This novel and unexpected finding suggests that homologous symbiont types (i.e. those usually found in a particular host species) may behave similarly and/or that the holobiont can adjust its physiology to allow for a shift in its symbiont population, so maintaining metabolic homeostasis. If so, this has major implications for the physiology and survival of reef corals under changing environmental regimes, and under future climate change scenarios where shifts in the resident symbiont population occur.

\subsection{Materials and Methods}

\subsubsection{Study design and location}

To determine the metabolite profiles associated with different, naturally-occurring Symbiodinium community compositions, I selected 40 M. capitata colonies in Kāne'ohe Bay, O'ahu, Hawai'i, USA, from three patch reefs located in the northern (Reef 44: $21^{\circ} 28^{\prime} 36.4^{\prime \prime} \mathrm{N}$, $157^{\circ} 50^{\prime} 01.0^{\prime} \mathrm{W}$ ), central (Reef 25: $21^{\circ} 27^{\prime} 40.3^{\prime \prime} \mathrm{N}, 157^{\circ} 49^{\prime} 20.1^{\prime \prime} \mathrm{W}$ ) and southern (HIMB [Hawai'i Institute of Marine Biology]: $21^{\circ} 26^{\prime} 06.0^{\prime \prime} \mathrm{N}, 157^{\circ} 47^{\prime} 27.9^{\prime}$ ' W) regions of the Bay (Figure 5.1). Samples were taken from the three separate reefs to increase the likelihood of sampling different Symbiodinium community compositions, at 1-3 m depth along a 50-100 m transect following the reef edge ( $n=40$ colonies total). These colonies have been tagged and the Symbiodinium composition repeatedly sampled since 24 October 2014, for a study on coral bleaching and recovery patterns (Cunning et al. 2016). The colonies were therefore known to possess a variety of Symbiodinium community compositions: clade C only; clade D only; or a mixture of clades $\mathrm{C}$ and $\mathrm{D}$. 


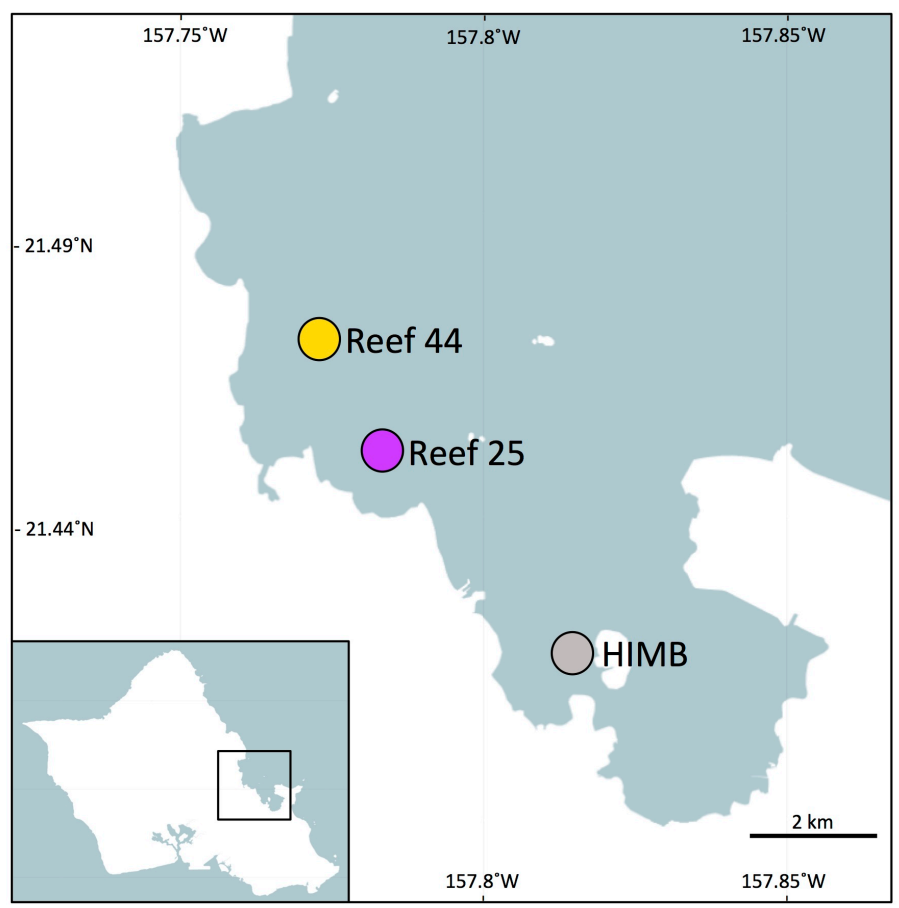

Figure 5.1 Study reef locations in Kāne'ohe Bay, O'ahu, Hawai'i, USA. HIMB: Hawai'i Institute of Marine Biology. Insert: Island of O'ahu, Hawai'i, USA, with Kāne'ohe Bay selected.

\subsubsection{Reef temperatures}

Temperature data were collected at $2 \mathrm{~m}$ depth at each reef site, using calibrated HOBO Pendant UA-002-08 loggers (Onset Corp., Bourne, MA, USA) (Ritson-Williams and Gates 2016). Daily mean values for temperature values $\left({ }^{\circ} \mathrm{C}\right)$ were calculated for each reef for two months prior to sampling $\left(20^{\text {th }}\right.$ July $2015-21^{\text {st }}$ September 2015). Data were analysed with a repeatedmeasures ANOVA (RMANOVA; SPSS statistical software, v. 20, IBM Corp), to test for differences in water temperature between sites that might influence coral metabolite profiles.

\subsubsection{Metabolite sample collection}

Single small branch fragments $(\sim 5 \times 5 \mathrm{~cm})$ of the coral $M$. capitata were removed from the unshaded upper surface of each colony sampled $\left(20^{\text {th }}\right.$ September $2015 ; n=40$ total fragments; Permit SAP-2016-58). A small $\left(\sim 1 \mathrm{~cm}^{2}\right)$ subsample of each colony was removed and placed in a $50 \mathrm{~mL}$ Falcon tube containing $50 \mathrm{~mL}$ seawater, for pulse amplitude modulated (PAM) measurements (Section 5.2.4). Metabolite samples were quickly transferred to a sterile Whirlpak bag (Micro-Analtyix, Auckland, NZ), sealed, and submersed in liquid nitrogen. The samples were frozen within $\sim 30 \mathrm{~s}$ of initial sampling. Samples were stored at $-80^{\circ} \mathrm{C}$ until transport via dry shipper to Victoria University of Wellington for fragment processing. 


\subsubsection{Photosynthesis indicators}

Dark-acclimated maximum quantum yield of photosystem II $\left(\mathrm{F}_{\mathrm{v}} / \mathrm{F}_{\mathrm{m}}\right)$ was measured with a diving PAM fluorometer (Walz, Effeltrich, Germany), following 30-min dark-acclimation of a small $\left(\sim 1 \mathrm{~cm}^{2}\right)$ fragment subsampled from each metabolite sample prior to quenching (see Section 5.2.3). PAM settings were maintained at: measuring light 4 , saturation intensity 8 , saturation width $1 \mathrm{~s}$, gain 4 and damping 2. Measurements were taken a standard distance of 5 $\mathrm{mm}$ from each sample. Mean estimates with standard error (S.E.M.) were calculated based on triplicate measurements from each sample. These fragments were not used for metabolite profile analysis. To test for differences in $\mathrm{F}_{\mathrm{v}} / \mathrm{F}_{\mathrm{m}}$ between the treatments groups, assumptions of normality and homoscedasticity were assessed using Shapiro-Wilk and Levene's tests, respectively, and non-normal or heteroscedastic data were $\log _{10}$-transformed prior to analysis with ANOVA at the $p<0.05$ probability level using SPSS statistical software (v. 20, IBM Corp).

\subsubsection{Metabolite sample processing}

All sample-processing steps were performed at $4^{\circ} \mathrm{C}$. Coral fragments were first defrosted on ice. Tissue was then removed from the skeleton via airbrush into a sterile Whirl-pak bag into 5 $\mathrm{mL}$ chilled MQ at $4^{\circ} \mathrm{C}$. The tissue homogenates were moved to $15 \mathrm{~mL}$ Falcon tubes and mechanically homogenised rapidly using a saw-tooth homogeniser (IKA T10 BS5, ThermoFisher Scientific, Auckland, New Zealand) for $1 \mathrm{~min}$ at a mid-speed setting. Each sample was thoroughly vortexed, and a single $500 \mu \mathrm{L}$ aliquot removed and stored at $-20^{\circ} \mathrm{C}$ for the final Symbiodinium cell density, protein content and chlorophyll $a$ measurements (see Sections 5.2.6 and 5.2.7).

Symbionts were pelleted by centrifugation $\left(2,500 \times \mathrm{g}\right.$ for $5 \mathrm{~min}$ at $\left.4^{\circ} \mathrm{C}\right)$. The supernatant comprised of host material was transferred and diluted with $5 \mathrm{~mL}$ of cold MilliQ water, followed by vigorous vortexing for $1 \mathrm{~min}$ and a second centrifugation $(2,500 \mathrm{x} g$ for $5 \mathrm{~min}$ at $\left.4^{\circ} \mathrm{C}\right)$ to remove residual symbiont cells $\left(2,500 \mathrm{x} g\right.$ for $5 \mathrm{~min}$ at $\left.4^{\circ} \mathrm{C}\right)$. Each symbiont pellet fraction was washed with $5 \mathrm{~mL}$ cold $\left(4^{\circ} \mathrm{C}\right)$ MilliQ water, the tube inverted, and a $500 \mu \mathrm{L}$ aliquot collected, to which $500 \mu \mathrm{L}$ DNA buffer $(0.4 \mathrm{M} \mathrm{NaCl}, 0.05 \mathrm{M}$ EDTA) with $1 \%(\mathrm{w} / \mathrm{v})$ sodium dodecyl sulphate (SDS) were added and the samples frozen at $-20^{\circ} \mathrm{C}$ for symbiont identification (Section 5.2.8). The remaining symbiont fraction was centrifuged $(2,500 \mathrm{x} g$ for 5 min at $4^{\circ} \mathrm{C}$ ) to remove residual host material. The cleaned host and symbiont fractions were frozen at $-80{ }^{\circ} \mathrm{C}$ for $1 \mathrm{~h}$ and lyophilised (FreeZone ${ }^{\circledR}$ Plus ${ }^{\mathrm{TM}} 4.5 \mathrm{~L}$ Cascade, Labconco, Auckland, New Zealand) for $18 \mathrm{~h}$ at $-105{ }^{\circ} \mathrm{C}$. Once thoroughly dry, $15 \mathrm{mg}$ of the symbiont 
fraction and $30 \mathrm{mg}$ of the host fraction were weighed out for metabolite extraction (Section 5.2.9).

\subsubsection{Quantification of symbiont cell density}

The algal pellet and host supernatant were separated by centrifugation $(3,000 \times \mathrm{g}$ for $10 \mathrm{~min})$. The Symbiodinium cells were re-suspended in $1 \mathrm{~mL}$ FSW and quantified using Improved Neubauer haemocytometer counts (Boeco, Germany) with a minimum of ten replicate counts per sample (i.e. to a confidence interval below 10\%). Cell density was normalised to soluble host protein content of the host fraction (triplicate measurements per sample), which was assessed by the Bradford assay (Bradford 1976). To test for differences in Symbiodinium cell densities between the treatments groups, assumptions of normality and homoscedasticity were assessed using Shapiro-Wilk and Levene's tests, respectively. As the data failed to meet the assumptions of normality, data were assessed using the non-parametric Kruskal-Wallis test at the $p<0.05$ probability level using SPSS statistical software (v. 20, IBM Corp).

\subsubsection{Chlorophyll a content}

Symbiont chlorophyll $a(\mathrm{Chl} a)$ content was quantified with approximately 10,000 cells (based on symbiont cell density calculated in Section 5.2.6) by N, N-dimethylformamide (DMF) extraction carried out in darkness over $48 \mathrm{~h}$ at $4^{\circ} \mathrm{C}$. Extracts were then centrifuged $(16000 \times \mathrm{g}$ for $5 \mathrm{~min}$ ) and the absorbance of triplicate $200 \mu \mathrm{L}$ aliquots was measured in 96-well plates (UVStar, Greiner Bio-One GmbH, Germany) at $646.8 \mathrm{~nm}, 663.8 \mathrm{~nm}$ and $750 \mathrm{~nm}$ using a microplate reader (Enspire ${ }^{\circledR} 2300$, Perkin-Elmer, Waltham, MA, USA). Chl $a$ concentrations were determined after optical path length correction $(0.555 \mathrm{~cm})$ using the equations of Porra et al. (1989). To test for differences in Chl a concentrations between the treatments groups, assumptions of normality and homoscedasticity were assessed using Shapiro-Wilk and Levene's tests, respectively, and analysed with ANOVA at the $p<0.05$ probability level using SPSS statistical software (v. 20, IBM Corp).

\subsubsection{Symbiont community identification}

DNA was extracted using the CTAB-chloroform extraction protocol (dx.doi.org/10.17504/protocols.io.dyq7vv). Quantitative PCR was used to determine the proportion of clade C and D Symbiodinium in each sample. Assays quantifying specific actin loci in clade C and D Symbiodinium were performed following the methods of Cunning and Baker (2013). Based on both internal transcribed spacer 2 (ITS2) and actin gene sequencing conducted by Cunning et al (2016), only specific clade C- and D-types were expected, for 
which target specificity of clade level primers was confirmed (Cunning et al. 2016). The multiplexed Symbiodinium clade C and D assay included $50 \mathrm{nM}$ clade C forward primer (CActF, 5-CCAGGTGCGATGTCGATATTC-3), $75 \mathrm{nM}$ clade $\mathrm{C}$ reverse primer (CactR, 5TGGTCATTCGCTCACCAATG-3), $100 \mathrm{nM}$ clade C Taqman probe (CactProbe, 5 -VICAGGATCTCTATGCCAACG-MGB-3), $50 \mathrm{nM}$ clade D forward primer (DactF, 5GGCATGGGGTAAGCACTTCTT-3), 75 nM clade D reverse primer (DactR, 5GATCCTTGAACTAGCCTTGGAAAC-3) and $100 \mathrm{nM}$ clade D Taqman probe (DactProbe, 5 -6FAM-CAAGAACGATACCGCC-MGB-3).

Thermal cycling conditions consisted of initial incubation at $50^{\circ} \mathrm{C}$ for 2 min and $95^{\circ} \mathrm{C}$ for 10 min, followed by 40 cycles of denaturation at $95^{\circ} \mathrm{C}$ for $10 \mathrm{~s}$ and annealing/extension at $60^{\circ} \mathrm{C}$ for $1 \mathrm{~min}$. Cycle threshold $(C t)$ values were calculated by the StepOnePlus software package using automatic baseline calculations and a set fluorescence threshold $\left(\Delta R_{n}\right)$ of 0.01 . Samples were run in duplicate, and positive amplifications were counted when both technical replicates produced $C t$ thresholds $<40$ and when no-template reactions showed no amplification. $C t$ values were normalised for variation in fluorescence intensity between the two reporter dyes $\left(\mathrm{FAM}^{\mathrm{TM}}\right.$ and VIC®) calculated from average $C t$ values of copy number standard curves (ranging from $10^{1}$ to $10^{6}$ ), which revealed that clade $\mathrm{C}(\mathrm{VIC} \AA)$ probe fluoresced $2.28 \pm 0.29$ (S.E.M.) cycles higher than the clade D $\left(\mathrm{FAM}^{\mathrm{TM}}\right)$ probe. Differences in the Symbiodinium composition among reefs were assessed by chi-squared test.

\subsubsection{Intracellular metabolite extraction}

Extraction was based on the methods in Chapter 4. Once thoroughly dry, $1 \mathrm{~mL} 100 \%$ methanol $\left(-20{ }^{\circ} \mathrm{C}\right)$ containing the internal standard (IS) D-sorbitol-6- ${ }^{13} \mathrm{C}$ (Sigma Aldrich, Auckland, New Zealand) was added to $30 \mathrm{mg}$ lyophilised host material to extract the semipolar metabolites. The host fraction was sonicated for $30 \mathrm{~min}$ at $4{ }^{\circ} \mathrm{C}$ in an ultrasonic bath (model: 8854 , Cole Parmer, USA). To the symbiont fraction, $200 \mu \mathrm{L} 100 \%$ methanol $\left(-20^{\circ} \mathrm{C}\right.$ and with IS) were added to $15 \mathrm{mg}$ lyophilised symbiont material, and symbiont cell walls were lysed by bead-milling with acid-washed glass beads ( $\sim 10 \mathrm{mg}$ of $710-1180 \mu \mathrm{m}$; Sigma-Aldrich, Auckland, New Zealand). Bead-milling was at a frequency of $50 \mathrm{~Hz}$ for $3 \mathrm{~min}$ (Tissuelyser II, Qiagen Inc., Hilden, Germany). A further $800 \mu \mathrm{L} \mathrm{MeOH}$ (at $-20^{\circ} \mathrm{C}$ with IS) were added to the lysed cells. Both fractions were centrifuged $\left(3,000 \mathrm{x} g\right.$ for $30 \mathrm{~min}$ at $\left.4^{\circ} \mathrm{C}\right)$, and the supernatant containing the extracted metabolites was collected and stored at $-80{ }^{\circ} \mathrm{C}$. Extraction was then repeated for both the host and symbiont material with $1 \mathrm{~mL} 50 \% \mathrm{MeOH}\left(-20{ }^{\circ} \mathrm{C}\right)$ to extract the polar fraction. Samples were then centrifuged $\left(3,000 \times \mathrm{g}\right.$ for $30 \mathrm{~min}$ at $\left.4^{\circ} \mathrm{C}\right)$ and the supernatant 
pooled with the first extract. The total extract was then centrifuged at $16,100 \mathrm{x} g$ for $15 \mathrm{~min}$ at $4^{\circ} \mathrm{C}$ to ensure the removal of all particulates. Aliquots (total volume $200 \mu \mathrm{L}$ host, $150 \mu \mathrm{L}$ symbiont) were then concentrated under vacuum (Eppendorf Concentrator 5301, Global Science, Auckland, New Zealand) at $30{ }^{\circ} \mathrm{C}$ until dry. The cell debris left over from both fractions was frozen at $-20^{\circ} \mathrm{C}$ for protein quantification, as determined by a modified Bradford colorimetric method (Smart et al. 2010) with BSA as the standard (Bradford 1976).

Trimethylsilyl ester derivatisation of sugars, sugar alcohols and amino/non-amino acids for GC-MS was performed using an automated on-line derivatisation, adapted from established protocols (Roessner et al. 2000). Briefly, all samples were re-dissolved in $20 \mu \mathrm{L}$ of $30 \mathrm{mg} \mathrm{mL}^{-1}$ methoxyamine hydrochloride in pyridine and derivatised at $37{ }^{\circ} \mathrm{C}$ for 120 min with mixing at $500 \mathrm{rpm}$. Twenty microlitres N,O-bis-(trimethylsilyl)trifluoroacetamide (BSTFA) and $1 \mu \mathrm{L}$ retention-time standard mixture comprised of $n$-alkanes were then added and the samples incubated for a further 30 min with mixing at $500 \mathrm{rpm}$. Each derivatised sample was then allowed to rest for $60 \mathrm{~min}$ prior to injection into the GC-MS. GC-MS analysis was performed on an Agilent 7890 gas chromatograph equipped with a Gerstel MPS2 multipurpose sampler and coupled to an Agilent 5975C VL mass selective detector, run in splitless mode, with an injection volume of $1 \mu \mathrm{L}$ of each sample and a technical replicate of two injections per sample. Instrument control was performed with Agilent G1701A Revision E.02.01 ChemStation software. The gas chromatograph was fitted with a Varian Factor 4 column (VF-5ms; $30 \mathrm{~m} \times$ $0.25 \mathrm{~mm} \times 0.25 \mathrm{um}+10 \mathrm{~m}$ Ezi-guard). Helium (Ultra High Purity) was in constant flow mode at approximately $1 \mathrm{~mL} \mathrm{~min}^{-1}$ with retention-time locking applied. The oven temperature was started at $70^{\circ} \mathrm{C}$, held at this temperature for $1 \mathrm{~min}$ and then increased at $7^{\circ} \mathrm{C} \min ^{-1}$ to $325^{\circ} \mathrm{C}$, and finally held at this temperature for $3.5 \mathrm{~min}$.

The mass spectrometer quadrupole temperature was set at $150^{\circ} \mathrm{C}$, with the source set at $250^{\circ} \mathrm{C}$ and the transfer line held at $280^{\circ} \mathrm{C}$. Positive ion electron impact spectra at $70 \mathrm{eV}$ were recorded in scan mode with the following settings: a gain factor of 1.00, detector threshold of 150, mass to charge $(\mathrm{m} / \mathrm{z})$ range of $50-600 \mathrm{~m} / \mathrm{z}$ and solvent delay of $6.0 \mathrm{~min}$. Along with the samples, a series of known metabolite mixtures and solvent-only blanks were analysed. The metabolite mixtures contained the major compound classes being targeted, including amino acids (Amino Acid Standard solution AAS18, Sigma-Aldrich, Auckland), fatty acids (Menhaden fish oil, Sigma-Aldrich, Auckland), and sugars (2.5 $\mu \mathrm{M}$ ribitol, ribose, fructose, glucose, arabinose, galactose, mannitol, xylose, mannose and sucrose, Sigma-Aldrich, Auckland), and were analysed to confirm that these compounds could be accurately identified. 


\subsubsection{Metabolite data extraction, pre-processing, normalisation and statistical analysis}

Metabolite data extraction and analysis were performed with the use of AMDIS (http://chemdata.nist.gov/mass-spc/amdis/) and Agilent MassHunter Workstation Software, Quantitative Analysis, Version B.05.00/Build 5.0.291.0. Compound identification was based on an in-house library of MS spectra, with retention indices based on n-alkane and fatty acid standards (Menhaden fish oil; Sigma-Aldrich, Auckland). Derivative peak area was used to semi-quantify the concentrations of individual metabolites. Data were normalised to the final area of the IS D-sorbitol- $6-{ }^{13} \mathrm{C}$ and the protein content of the cell debris resulting from the samples' intracellular metabolite extraction using a modified Bradford colorimetric method (Smart et al. 2010) (Section 5.2.9). The host metabolite profiles were then normalised to Symbiodinium cell density to account for differences between samples and hence translocation of photosynthetic products, and the symbiont metabolite profiles to symbiont cell number (Section 5.2.6).

Data were then tested for normality and homogeneity, and $\log _{2}$ transformed where necessary. Data were then autoscaled, and statistical analysis was performed using MetaboAnalyst 3.0 (http://www.metaboanalyst.ca) (Xia et al. 2015). Relative metabolite concentrations of both the host and symbiont fractions were evaluated by Principle Component Analysis (PCA) and grouped according to Symbiodinium community composition. Significance Analysis of Microarrays (and Metabolites) (SAM) was performed to identify individual metabolites in the host and symbiont fractions that varied with Symbiodinium composition. SAM is designed to address the false discovery rate problems (FDR) that arise from running multiple tests on highdimensional data (Xia and Wishart 2011). Furthermore, it is considered robust for detecting differences between treatments with unequal small sample sizes and unequal variances in metabolomic datasets (Yang et al. 2006; Vinaixa et al 2012). It also implements permutation testing to reliably identify significant individual metabolites. It first assigns a significance score to each variable (metabolite) based on its change relative to the standard deviation of repeated measurements. Then it chooses variables with scores greater than a defined significance threshold (delta; d(i)) and compares their relative difference to the distribution estimated by random permutations of the class labels (Symbiodinium community composition). For each threshold, a certain proportion of the variables in the permutation set will be found to be significant by chance. The number is used to calculate the false detection rate (FDR). In this way, SAM is able to perform permutation testing, contrary to t-tests and ANOVAs. Significant individual metabolites were determined based on a FDR corrected significance value, $p<0.05$. 


\subsection{Results}

\subsubsection{Reef temperatures}

To test for any potential impacts of water temperature on metabolite profiles, I compared the temperature across the three sampling sites. Temperature was not different between reefs from $20^{\text {th }}$ July $2015-21^{\text {st }}$ September 2015 (RMANOVA, time $\mathrm{x}$ temperature, $F_{59,179}=0.544, p=$ 0.465) (Figure 5.2). All temperatures were between $28.15^{\circ} \mathrm{C}$ (Reef HIMB) and $28.42^{\circ} \mathrm{C}$ (Reef $44)$ at the time of sample collection.

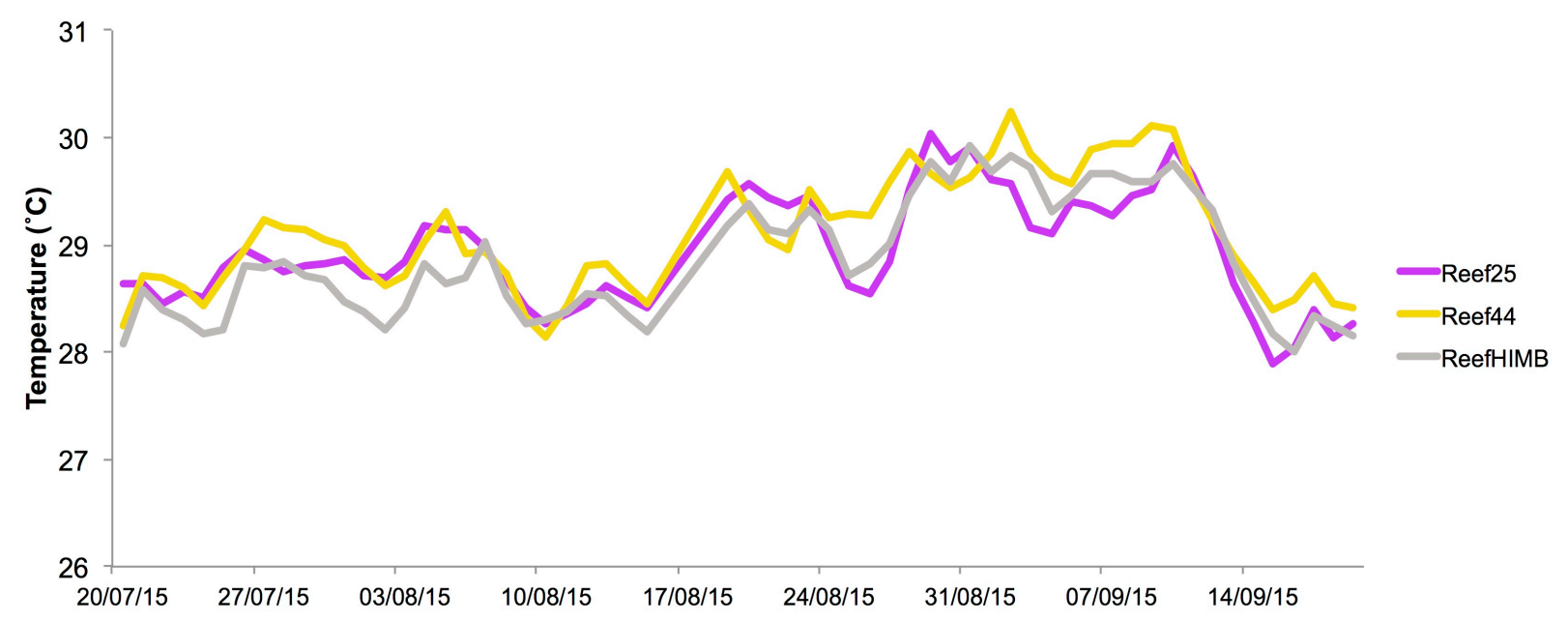

Figure 5.2 Daily mean temperatures from the three reef locations for two months prior to sample collection.

\subsubsection{Symbiodinium community structure}

Quantitative PCR assays detected clade C and D Symbiodinium either alone or in mixed communities (Table 5.1). Across all colonies, 50\% contained C only, $42.5 \%$ contained both $\mathrm{C}$ and D, whilst only 7.5\% contained D only. All four Symbiodinium combinations were observed at Reefs 25 and 44, while D-only colonies were not observed at Reef HIMB. The Symbiodinium community compositions were not significantly different between the reef locations (Figure 5.3) (Chi-square test, $X^{2}=1.56, p=0.955$ ). All reefs were predominantly clade C-only, with $60 \%, 44 \%$ and $43 \%$ hosting clade C-only at Reef HIMB, Reef 25 and Reef 44 , respectively. 
Table 5.1 Quantitative PCR results for the Symbiodinium community composition of the M. capitata samples. Values are number of colonies within each reef that host each of the different symbiont community types, plus the total for each symbiont community type across all three reef sites. Labels indicate Symbiodinium composition: $\mathrm{C}=$ clade C-only; $\mathrm{Cd}=$ clade $\mathrm{C}$ dominant with less abundant clade $\mathrm{D} ; \mathrm{Dc}=$ clade $\mathrm{D}$ dominant with less abundant clade $\mathrm{C} ; \mathrm{D}=$ clade D-only.

\begin{tabular}{llll|l}
\hline Symbiont composition & Reef HIMB & Reef 25 & Reef 44 & Total \\
\hline C & 7 & 7 & 6 & 20 \\
Cd & 1 & 3 & 2 & 6 \\
Dc & 2 & 4 & 5 & 11 \\
D & 0 & 2 & 1 & 3 \\
\hline Total & 10 & 16 & 14 & 40 \\
\hline
\end{tabular}

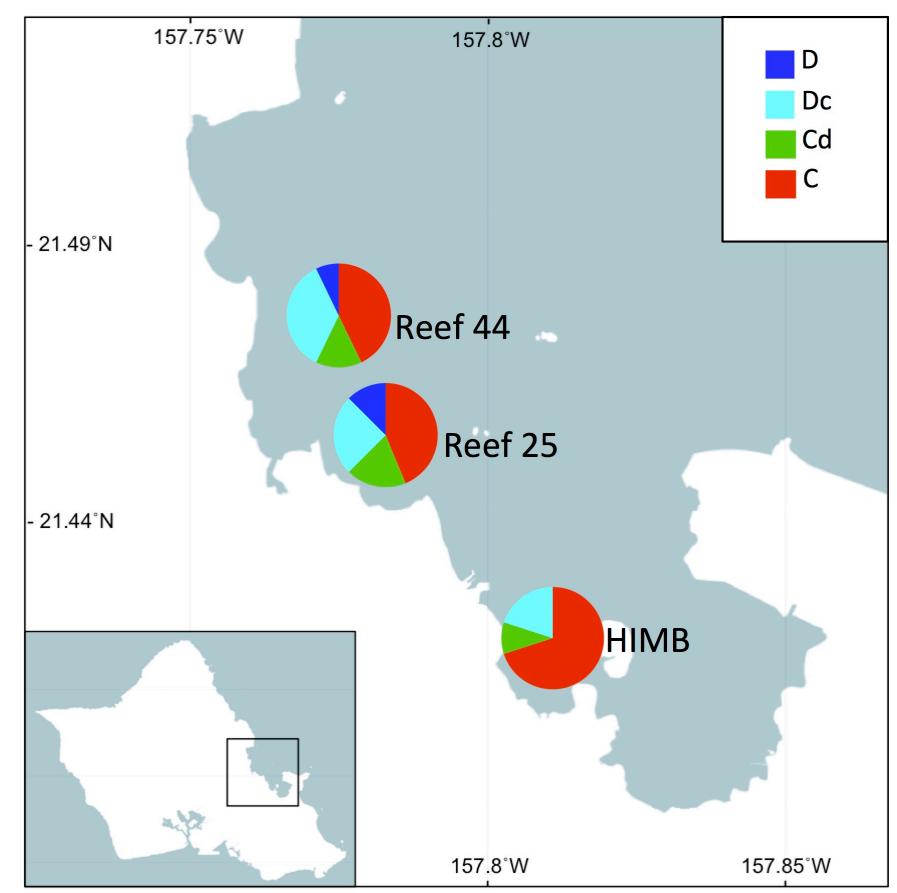

Figure 5.3 Spatial patterns in Symbiodinium community composition at each of the reef sites in Kāne'ohe Bay, O'ahu, Hawai'i, USA. HIMB: Hawai'i Institute of Marine Biology. Insert: Island of O'ahu, Hawai'i, USA, with Kāne'ohe bay selected. Labels indicate Symbiodinium composition: $\mathrm{C}=$ clade $\mathrm{C}$-only; $\mathrm{Cd}=$ clade $\mathrm{C}$ dominant with less abundant clade $\mathrm{D} ; \mathrm{Dc}=$ clade D dominant with less abundant clade $\mathrm{C} ; \mathrm{D}=$ clade $\mathrm{D}$-only.

The similar temperature at each reef location suggests that this environmental variable is unlikely to contribute to any differences seen in the metabolite analysis. Furthermore, the host and symbiont metabolite profiles were compared between the three reef locations using PCA and SAM analyses, and were found to be unaffected by site (Figure S5.1). As a result, I pooled samples across the reef sites to provide the minimum sample size of $n=3$ recommended for metabolomics analyses (Villas-Boas et al. 2007; Grootveld 2012). 


\subsubsection{Photo-biological and symbiosis biomass parameters}

Various photo-biological and biomass parameters of different Symbiodinium communities were measured as a proxy for photosynthetic capacity (Table 5.2). The maximum photosynthetic yield of photosystem II $\left(\mathrm{F}_{\mathrm{v}} / \mathrm{F}_{\mathrm{m}}\right)$ did not differ between these different communities (one-way ANOVA, $\left.F_{3,39}=1.434, p=0.249\right)$. Furthermore, chlorophyll $a$ concentration per cell and symbiont cell density did not differ significantly between the different communities (one-way ANOVA, $F_{3,39}=1.443, p=0.246$ and Kruskal-Wallis, $H_{3,39}=5.294, p=0.152$, respectively).

Table 5.2 Photobiological characteristics of individual $M$. capitata colonies hosting different Symbiodinium community compositions. Data are means \pm S.E.M. Labels indicate Symbiodinium composition: $\mathrm{C}=$ clade C-only $(n=20)$; $\mathrm{Cd}=$ clade $\mathrm{C}$ dominant with less abundant clade $\mathrm{D}(n=6) ; \mathrm{Dc}=$ clade $\mathrm{D}$ dominant with less abundant clade $\mathrm{C}(n=11) ; \mathrm{D}=$ clade D-only $(n=3)$.

\begin{tabular}{lllll}
\hline Parameter & C & Cd & Dc & D \\
\hline $\mathrm{F}_{\mathrm{v}} / \mathrm{F}_{\mathrm{m}}$ & $0.469 \pm 0.034$ & $0.573 \pm 0.045$ & $0.537 \pm 0.040$ & $0.512 \pm 0.028$ \\
$\mathrm{Chl} a$ content $\left(\mathrm{pg} \mathrm{cell}{ }^{-1}\right)$ & $4.00 \pm 0.55$ & $4.13 \pm 0.66$ & $5.16 \pm 0.66$ & $2.30 \pm 0.66$ \\
$\begin{array}{l}\text { Symbiodinium cell } \\
\text { density (cells } \mu \mathrm{g}^{-1}\end{array}$ & $64.73 \pm 12.63$ & $56.91 \pm 9.39$ & $105 \pm 19.34$ & $63.77 \pm 22.21$ \\
protein) & & & & \\
\hline
\end{tabular}

\subsubsection{Host metabolite pools}

Non-targeted GC-MS analysis identified 54 metabolites in $M$. capitata host tissues, including carbohydrates, amino acids, fatty acids, lipogenesis intermediates and organic acids (Table S5.1). The most abundant metabolite was scyllo-inositol. There were 15 metabolites that were distinctive of the host metabolite profiles (i.e. the metabolite was not detected in symbiont pools): 1-octadecanol, cholesterol, citric acid, fructose, glutamine, guanosine, isoleucine, nicotinic acid, phenylalanine, ribose, ribose-5-phosphate, ribonic acid, succinic acid, valine and xylose.

PCA analysis of the host metabolite profiles revealed no apparent spatial separation of the samples relative to the Symbiodinium community composition (Figure 5.4A). All groups and the 95\% confidence intervals show substantial overlap along both PC axes 1 and 2 (52\% and $17.7 \%$ of the explained variation, respectively). Furthermore, Significance Analysis of Microarrays (and Metabolites) (SAM) reported that no individual metabolites differed in response to symbiont composition (SAM, $p>0.05$, FDR $>0.05$; Figure 5.4B). 


\subsubsection{Symbiont metabolite profiles}

Non-targeted GC-MS analysis identified 43 metabolites in symbiont pools, largely consisting of sugars, amino acids, fatty acids and organic acids (Table S5.2). Those metabolites distinctive of the symbiont metabolite profiles (i.e. metabolites not detected in the host pools) included: aspartic acid, dodecanoic acid (C12:0), fumaric acid, galactonic acid, linoleic acid (C18:2n-6) and gamma-linolenic acid (C18:3n-6). The most abundant metabolite was galactonic acid.

Symbiont metabolite profiles showed no distinctive spatial separations in response to Symbiodinium community composition (Figure 5.4C). The distribution of samples was similar along both PC axes 1 and 2, accounting for 43.3 and $13.7 \%$ of the explained variation, respectively. SAM analysis did not identify any metabolites in the symbiont pools that were different in the different symbiont communities ( $p>0.05$, FDR $>0.05$; Figure 5.4D). 
A

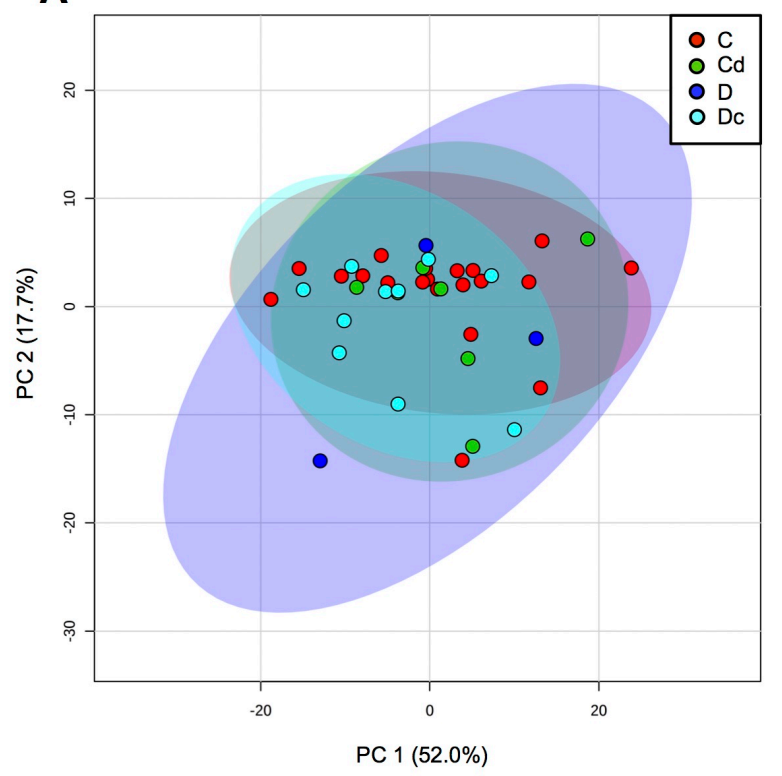

C

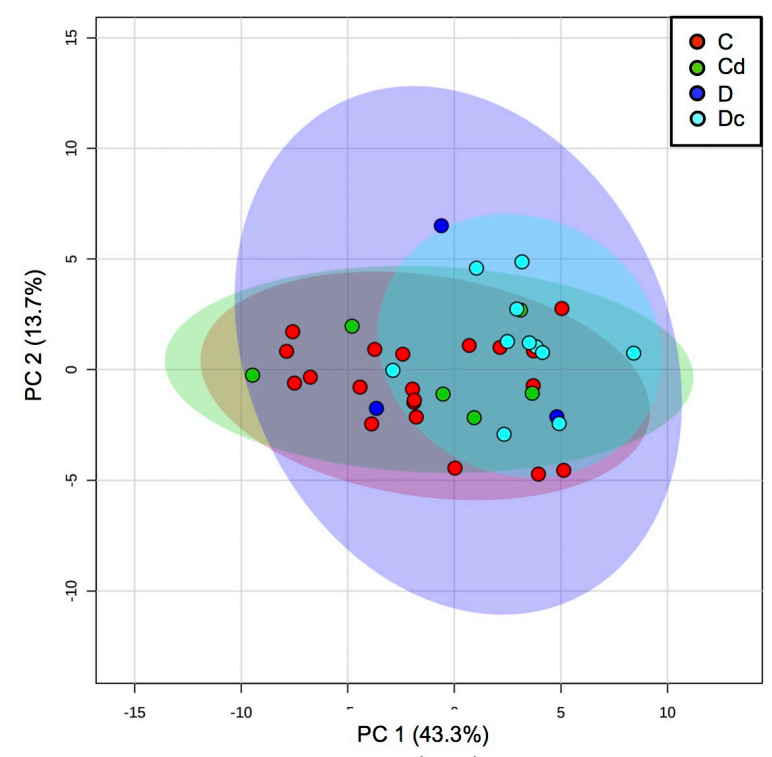

B
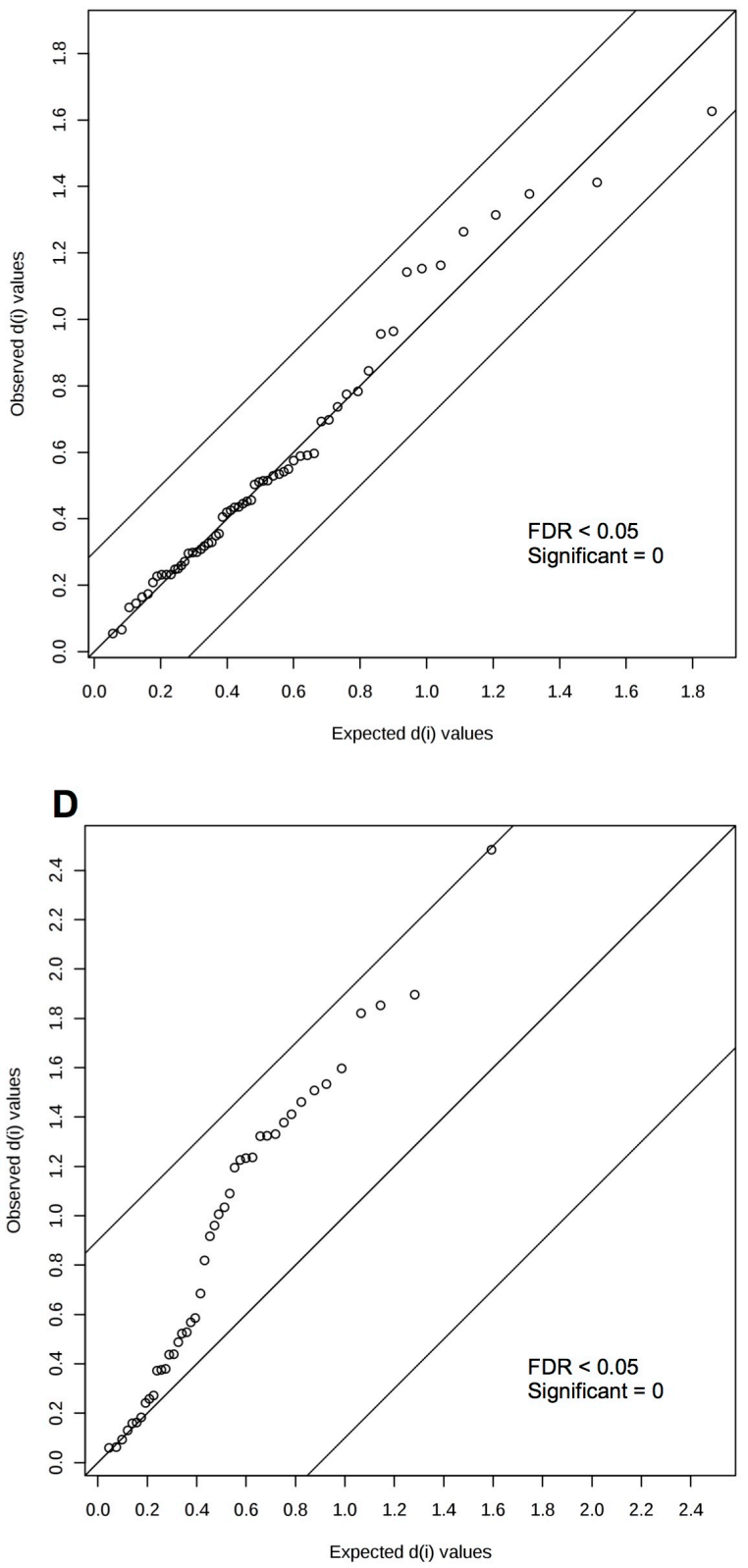

Figure 5.4 Host and symbiont metabolite profiles grouped according to Symbiodinium community composition, with $\mathbf{9 5 \%}$ confidence intervals. A) PCA analysis of the relative abundance of the metabolites detected in host free-metabolite pools in response to the four different Symbiodinium community compositions; B) SAM analysis for the identification of individual metabolites that may vary between the symbiont community types in the host metabolite profiles. C) PCA analysis of the relative abundance of the metabolites detected in symbiont free-metabolite pools in response to the four different Symbiodinium community compositions (with 95\% confidence regions); D) SAM analysis for the identification of individual metabolites that may vary between the symbiont community types in the symbiont metabolite profiles. Labels indicate Symbiodinium composition: C = clade C-only $(n=20)$; Cd $=$ clade $\mathrm{C}$ dominant with less abundant clade $\mathrm{D}(n=6)$; $\mathrm{Dc}=$ clade $\mathrm{D}$ dominant with less abundant clade $\mathrm{C}(n=11) ; \mathrm{D}=$ clade $\mathrm{D}$-only $(n=3)$. 


\subsection{Discussion}

Flexibility of the Symbiodinium community composition plays a role in the ability of corals to withstand environmental change, although there may be nutritional consequences of a change to the symbiont community that cause it to become less beneficial. In such a case, there may be a "trade-off" between this loss of nutritional potential and the advantage of being able to persist in a changing environment, though it is unclear what the longer-term consequences of this loss in nutritional potential might be (Ortiz et al. 2013; Cunning et al. 2015a), or indeed whether the symbiotic interaction state (i.e. whether it is more or less beneficial) is fixed over time. The results of the current study contribute to our understanding of this topic and provide a platform for further studies of shifts in the nutritional potential of corals, by demonstrating that the metabolite profiles of the host and the symbionts are similar in M. capitata under 'normal' environmental conditions, irrespective of whether it is dominated by Symbiodinium clade $\mathrm{C}$ or $\mathrm{D}$, clades that are widely considered to be more and less nutritionally beneficial partners of reef corals, respectively.

\subsubsection{Symbiont community composition does not affect the metabolite profile of Montipora capitata}

M. capitata in Kāne'ohe Bay formed a symbiosis with either clade C or clade D Symbiodinium, or both clades simultaneously, with either clade C-dominance or clade D-dominance. On both a global scale and specifically in Kāne'ohe Bay, clade D-associated M. capitata is currently understood to be extremely rare (Franklin et al. 2012; Cunning et al. 2016), which corresponds with the data here. Nevertheless, the similar symbiont cell densities and photo-biological performance seen here suggest that all the coral colonies were in a similarly functional and stable symbiosis at the time of sampling, regardless of the Symbiodinium community composition. These results are consistent with prior evidence of the presence and stability of these different host-symbiont pairings in Kāne'ohe Bay (LaJeunesse and Thornhill 2011; Franklin et al. 2012; Stat et al. 2013; Cunning et al. 2016). The apparent stability of the different host-symbiont combinations was further supported by there being no variation in the identity or quantity of free metabolite pools in either the host tissues or symbionts in the different host-symbiont pairings. This latter finding was somewhat surprising, as symbiont diversity has previously been linked to differences in the translocated compounds derived from photosynthesis, including sugars and amino acids, in different host species (Trench 1979; Miralles et al. 1989), while certain symbiont types translocate relatively little photosynthate (Loram et al. 2007; Cantin et al. 2009; Starzak et al. 2014). Furthermore there is evidence for 
taxonomic variation in the metabolite profiles of Symbiodinium cells in culture (Klueter et al. 2015). Of particular relevance to the current study, an association with clade D under normal conditions was shown to come at the cost of reduced carbon translocation (Cantin et al. 2009; Pernice et al. 2015), altered photokinetics and energetics (Jones and Berkelmans 2011; Jones and Berkelmans 2012), and ultimately reduced reproduction and growth of the coral host (Little et al. 2004; Jones and Berkelmans 2010; Cunning et al. 2015a). Why my results are inconsistent with these previous studies is uncertain, but there are three possible reasons, relating to the host, symbiont or both in combination: 1) the host may compensate for a reduced nutritional input from its symbionts via increased heterotrophy; 2) the identity and quantity of translocated photosynthetically-fixed metabolites may be controlled by the symbiont community and/or the host, perhaps through selective pressures acting to optimise the symbiosis over time; and/or 3) metabolic pathway activity may differ between the associations, but yield similar profiles of the free-metabolites. These potential explanations are discussed in more detail below.

\subsubsection{Host heterotrophic compensation}

The observed similarity in the free metabolite profiles of the host does not necessarily indicate similarity in the translocation of organic products from the algal symbionts. Indeed, symbiotic cnidarians rely not only on autotrophic sources of carbon from the symbiont, but also on heterotrophy, by feeding on planktonic organisms (Falkowski et al. 1984; Leal et al. 2015). This trophic plasticity is critical for the resilience of these symbiotic associations during environmental fluctuations (Grottoli et al. 2006; Hughes and Grottoli 2013). Therefore, host heterotrophy may act to compensate for any differences in the autotrophic contribution of different symbiont types, such as by increased feeding and digestion when in symbiosis with a less nutritionally beneficial symbiont type (Grottoli et al. 2006; Ferrier-Pagès et al. 2011; Hughes and Grottoli 2013). Indeed, this has recently been shown in Aiptasia sp. when in an association with heterologous clade D symbionts, i.e. symbionts that are not normally found in this host species (Leal et al. 2015). Similarly, given that the metabolite profiles of some Symbiodinium types differ in culture (Klueter et al. 2015), the lack of any variation in the symbiont metabolite profiles seen in the current study may be due to plasticity in the metabolites translocated from the host to the symbiont (Papina et al. 2003; Chen et al. 2015). However, heterotrophic compensation may come at a metabolic cost for the host, as heterotrophic processes are metabolically demanding (Sebens 2002), requiring mobilisation of energy stores (Rodrigues and Grottoli 2007) and, in the case of corals, the assimilation of photosynthetic carbon to support host respiration (Falkowski et al. 1984; Tremblay et al. 2016). 
Moreover, while heterotrophic carbon is used in host biomass, autotrophic carbon is used in energy production, so one may not directly compensate for the other (Bachar et al. 2007; Tremblay et al. 2015). Therefore, while host heterotrophic compensation may provide shortterm relief, it may ultimately come at a metabolic cost to the symbiosis that will likely affect the persistence of a sub-optimal host-symbiont pairing. If such events are occurring in the present study, then it could help to explain the relative scarcity of D-only corals given that symbionts of this clade have been shown to be relatively poor at meeting the nutritional demands of their host (Little et al. 2004; Jones and Berkelmans 2010; Cunning et al. 2015a). Measurement of heterotrophic uptake by the corals used in this study would help to elucidate this matter. However, if true, clade D-dominated hosts would have comparably fewer energy stores and reduced coral growth in the long-term, casting doubt over the ability of thermallytolerant clade D Symbiodinium to ameliorate the effects of global warming (Stat and Gates 2011; Ortiz et al. 2013; Ortiz et al. 2014; Cunning et al. 2015a).

\subsubsection{Modifications to the composition and quantity of translocated photosynthate by the symbionts and/or the host}

Taxonomic variation in the metabolite profiles of Symbiodinium in culture has been demonstrated (Klueter et al. 2015), however this does not necessarily mean that the metabolite profiles of different Symbiodinium types in hospite will differ. The similarity in the metabolite profiles irrespective of symbiont community composition may be due to adaptation in terms of the quality and quantity of synthesised and translocated photosynthetic metabolites, and may be due to two non-mutually exclusive reasons: (1) plasticity in the metabolites synthesised and translocated by the symbiont; and (2) host control of the synthesis and translocation of specific photosynthetic metabolites. In the case of the former, under similar environmental conditions, a single type of Symbiodinium may synthesise and translocate different metabolites depending on the host genotype. However, to the best of my knowledge this has not yet been investigated. Meanwhile, chemical signal(s) (so called "host release factors") and cell signalling molecules may be responsible for the release of photosynthate and control photosynthetic performance (Muscatine et al. 1972; Gates et al. 1995; Wang and Douglas 1997; Grant et al. 1998; Withers et al. 1998; Gates et al. 1999; Cook and Davy 2001; Grant et al. 2001; Grant et al. 2003; Grant et al. 2006b), and therefore may be responsible for homogeneity in the translocation of photosynthate irrespective of the symbiont type, providing similarity in the metabolite profiles detected here. However, a study by Sutton and Hoegh-Guldberg et al. (1990) demonstrated that different symbionts behave differently in the same host release factor (HRF). Furthermore, it is important to note that studies into the presence and role of HRF's in the symbiosis were carried 
out in vitro (as opposed to in hospite) (Muscatine 1967; Sutton and Hoegh-Guldberg 1990; Gates et al. 1995; Wang and Douglas 1997; Cook and Davy 2001), and so whether HRFs actually exist in hospite and are involved in the mechanisms regulating carbon translocation remains unclear. Moreover, neither explanation would be compatible with the differential rates of carbon translocation previously detected in clade C- and D-dominant corals (Little et al. 2004; Jones and Berkelmans 2010; Cunning et al. 2015a).

A more likely alternative is that the clade C and D symbionts have coevolved with the host, with the quality and/or quantity of metabolites exchanged being adjusted over time to optimise symbiotic performance, either at the individual cell or symbiont community level. Indeed, even subtle changes in mixed symbiont populations (e.g. dominant $\mathrm{C}$ compensating for background D) may optimise the symbiotic potential, and allow for the maintenance of metabolic homeostasis and stress tolerance (Loram et al. 2007; Cunning and Baker 2014; Cunning et al. 2015c). How such metabolic adjustments might occur is unknown, but could arise through direct selection on the symbionts by the host in favour of more beneficial members of the symbiont population or environmental selection on the holobiont based upon its overall metabolic performance (Thrall et al. 2006; Wolinska and King 2009). Similar mechanisms are thought to promote the selection of beneficial microbial symbionts, e.g. as occurred in the evolution of the dominant gut symbiont Bacteriodes thetaiotaomicron in humans (e.g. Ley et al. 2006). Moreover, the evolution of stable beneficial bacterial associations from parasitic lineages predominantly relies on vertical transmission (i.e. symbiont acquired directly from the parent) (Ott et al. 2014), and interestingly, the fitness of the jellyfish Cassiopea xamachana increases when its symbiont (Symbiodinium microadriaticum) is acquired via vertical transmission as opposed to horizontal acquisition (i.e. initially aposymbiotic host acquires symbionts from the environment) (Sachs and Wilcox 2006). The Hawaiian coral Montipora capitata used in the current study acquires its symbiont via vertical transmission (PadillaGamiño et al. 2012), and therefore host selective pressures acting on the symbiont community seems the most likely scenario to explain the selection and persistence of different beneficial host-symbiont combinations in this coral. This is a fascinating area for future study, and the measurement of metabolic fluxes over multiple generations of the host, for example with the use of stable isotope tracers, could shed some light on this matter. 
5.4.1.3. Compensatory adjustments to metabolic pathway activity may generate similar metabolite profiles in the presence of different symbiont types

While the free-metabolite profiles of both partners were indistinguishable between the different Symbiodinium community compositions, this does not necessarily indicate similarity in the metabolic pathway activity of the different associations. For example, given evidence that clade $\mathrm{C}$ symbionts translocate relatively more carbon than clade D symbionts (Cantin et al. 2009), and that coral growth is faster for Pocillopora damicornis dominated by clade C under normal conditions, than conspecifics dominated by clade D (Cunning et al. 2015a), metabolic pathway activity of the clade C-dominant hosts was perhaps directed towards growth and storage of metabolites. The increased turnover of pathways forming phospholipids, proteins and nucleic acids, and the increased production of glycogen stores in the clade C-dominated host tissues would consequently reduce the abundance of free-metabolite pools. Conversely, clade D-dominant corals receive relatively less carbon and exhibit slower growth rates (Jones and Berkelmans 2010; Jones and Berkelmans 2011; Jones and Berkelmans 2012), so the available nutrients were perhaps rapidly turned-over in glycolysis and energy-producing pathways to ensure metabolic homeostasis and functional cellular respiration in the host. Such compensatory adjustments to metabolic activity could ultimately lead to relative uniformity in the downstream metabolite profile of the host. However, different metabolic pathways require different substrates, and produce different intermediates and end-products, so if such differences in metabolic activity were occurring here, then some variation in the host's metabolite profiles would be expected; this was not seen.

The similarity of the symbiont metabolite profiles was at first surprising given the different profiles reported for different Symbiodinium types in culture (Klueter et al. 2015). However, given the similarity of the various photophysiological and symbiont cell density parameters measured, it is conceivable that cell proliferation, photosynthate production, symbiont cell metabolism, and the corresponding pathways, were potentially turning over at equivalent rates in the different symbioses, generating similar metabolite profiles. Much more detailed analysis of symbiont physiology is required, however, to determine whether this is truly the case.

\subsubsection{Metabolic homeostasis as a requirement for the persistence of different, and} potentially novel, host-symbiont pairings: the implications for holobiont adaptation

Over time, a coral species can establish symbioses with different symbiont types, and despite there being clear links between symbiont type and nutritional performance (Cantin et al. 2009; Starzak et al. 2014; Leal et al. 2015; Pernice et al. 2015), if the symbiont remains in symbiosis 
with a particular host for long enough, there may be adjustment of host and/or symbiont physiology to afford an optimal holobiont interaction. Of the scenarios presented above, host selective pressure acting on the symbiont community seems the most likely way that this could be achieved. This would in turn lead to a convergence of the metabolite pools in the different host-symbiont combinations. Subsequently, such physiological adjustments may facilitate the evolution of novel host-symbiont combinations, including associations with more thermotolerant but initially less nutritionally beneficial symbiont types, such as from clade D (Stat et al. 2011; Cunning et al. 2015b; Leal et al. 2015). In this regard, the capacity for the acquisition of novel clade $\mathrm{D}$ symbionts from the surrounding environment in adult colonies has recently been shown in Pocillopora damicornis and Stylophora pistillata colonies following two consecutive bleaching events (Boulotte et al. 2016). Although the newly acquired D types remained in low abundance during the study by Boulotte et al. (2016), over time, if the holobionts' metabolic homeostasis is maintained, host selective pressure may allow these symbionts to become dominant, particularly if they provide an ecological benefit when environmental conditions change. Ultimately, such shifts along the parasitism-mutualism continuum mean that mutualistic symbionts can evolve from opportunistic or parasitic symbionts, such as thermotolerant clade D Symbiodinium, and aid the future survival of coral reefs (Berkelmans and van Oppen 2006; Sachs et al. 2011; Stat et al. 2011; Boulotte et al. 2016).

\subsection{Conclusion}

Suboptimal symbioses between host and symbiont lineages that have not co-evolved may be a result of a difference in the identity of translocated material between different symbiont types (Chapter 4). However the evidence presented here suggests that flexible associations may have evolved mechanisms of coping with variable metabolic contributions, so countering the potential nutritional costs often associated with more thermotolerant Symbiodinium genotypes, such as those belonging to Clade D. This has important implications for our understanding of the potential for flexibility in the coral-Symbiodinium association to provide an adaptive mechanism in response to climate change. Indeed, ecosystem-scale modelling brings into question whether symbiont traits might ameliorate environmental change, if the physiological costs of hosting a particular symbiont type outweigh the ecological benefits (Ortiz et al. 2013; Ortiz et al. 2014). However, these models are based on studies demonstrating a negative effect of clade D on coral growth, a pattern that is evidently environment- and host species-dependent 
(Cunning et al. 2016). Nevertheless, more studies in other flexible coral-dinoflagellate associations, combined with the analysis of the wider metabolome including lipids and proteins, are required to support the findings presented here.

Determining the nutritional implications at the individual metabolite level, as demonstrated here, will help to elucidate the specific physiological costs and/or benefits associated with certain symbiont types. This study also highlights the requirement for studying host and symbiont metabolism in situ, rather than each partner in isolation. Clearly, metabolite profiling with a variety of environmental and host-symbiont combinations has to potential to elucidate the dynamic regulation of the symbiosis, and the adaptive potential of the association. 


\section{Chapter 6}

\section{General Discussion}

\subsection{Summary}

Currently, major gaps exist in our understanding of the nutritional flux between cnidarians and their phototrophic algal symbionts, but greater in-depth knowledge is of paramount importance for understanding the potential for coral reefs to adapt to environmental change. This thesis aimed to determine the molecular and metabolic implications of cnidarians harbouring different Symbiodinium types, to further our understanding of the potential for partner switching as a mechanism for corals to survive climate change. The development and optimisation of a method involving menthol-induced aposymbiosis was integral to this work (Chapter 2). Transcriptomic and metabolomic techniques were used to explore the molecular and metabolic pathway activity affected in the host tissues of a model symbiosis (Aiptasia sp.) when experimentally colonised with different Symbiodinium types, and in both symbiotic partners (symbiont and host) of a coral (Montipora capitata) that naturally harbours different Symbiodinium community compositions. Several important discoveries about the potential for the adaptation of flexible associations were made in this work: most importantly, that heterologous (i.e. not originally found in the host) Symbiodinium types affect the abundance and diversity of metabolites translocated to the host, and that the persistence of novel symbioses is likely to require modification to and/or compensation for the metabolic exchange between the partners (Chapters 3-5). This corroborates, and adds substantially to, previous research demonstrating that Symbiodinium genotypic diversity affects the quantity and identity of translocated material to the host (Trench 1971a; Miralles et al. 1989; Loram et al. 2007; Cantin et al. 2009; Starzak et al. 2014; Leal et al. 2015), and that coral's are able to compensate for reduced autotrophy through increased heterotrophy and catabolism of energy stores (Grottoli et al. 2006; Hughes and Grottoli 2013; Tremblay et al. 2014; Leal et al. 2015; Tremblay et al. 2015).

A further significant discovery arising from this work is that modulation of the oxidative environment is likely to influence the establishment, maintenance and breakdown of novel symbioses (Chapters 3 and 4). Pathway analysis revealed a disruption to the oxidative state in Aiptasia sp. when colonised with a heterologous symbiont (Chapter 3), and differences in the translocation of antioxidants and their precursors further supports a role for the oxidative environment as a mediator of partner communication that regulates the symbiosis (Chapter 4). This supports and builds upon evidence that symbiosis specificity and collapse are likely to 
involve similar mechanisms (Weis 2008; Schnitzler and Weis 2010; Davy et al. 2012; Detournay et al. 2012). Indeed, besides the role of reactive oxygen and nitrogen species (collectively abbreviated as RONS) in the bleaching cascade of the cnidarian-dinoflagellate symbiosis, successful infection of Aiptasia sp. with homologous symbionts results in a downregulation of host NO production pathways (Detournay et al. 2012), while infection of Aiptasia sp. with unsuitable or dysfunctional symbiont cells may lead to heightened host NO synthesis (Hawkins 2013). Furthermore, transcriptomic analysis revealed that symbiont tolerance likely involves complex changes required to handle the production of RONS by the symbionts (Mohamed et al. 2016). This response is compatible with mechanisms of establishing specificity in other obligate mutualisms, e.g. ROS regulation plays an important role in the tolerance of resident bacteria and elimination of pathogenic ones in Drosophila melanogaster (Ha et al. 2009; Moné et al. 2014).

Although the exact process is unclear, it is evident here (Chapters 3-5), and from our understanding of other symbioses (Davidson et al. 2004; Ha et al. 2009; Moné et al. 2014; Farnese et al. 2016), that optimal nutritional exchange and regulation of the oxidative environment are likely to play a critical role in the horizontal establishment and maintenance of novel cnidarian-dinoflagellate associations. From these findings, this thesis proposes a conceptual molecular model for the adaptation of novel host-symbiont pairings (Figure 6.1).

The cascade begins with nutritional exchange between the partners, whereby the host translocates sufficient dissolved inorganic carbon (DIC), organic and inorganic nitrogen (N) and phosphorus $(\mathrm{P})$ to the symbiont to support its metabolism and photosynthesis (Wang and Douglas 1999; Imbs et al. 2014; Ferrier-Pagès et al. 2016) . In return, the symbionts translocate photosynthetically-fixed carbon, including sugars, amino acids, fatty acids and lipids, contributing significantly to the host cnidarian's energetic budget, as well as promoting deposition of calcium carbonate skeletons in corals (Muscatine and Cernichiari 1969; Muscatine 1973; Muscatine and Porter 1977; Wang and Douglas 1999; Kopp et al. 2015). In a heterologous association, suboptimal or incompatible nutritional exchange from the symbiont results in the catabolism of energy stores (Chapter 4), which, combined with a host immune response to an unfamiliar symbiont (Mohamed et al. 2016), results in increased RONS generation (Chapter 3). The antioxidant repertoire of the host and/or symbiont may subsequently determine the dosage of ROS experienced by the cell and ultimately the molecular response. At low doses, gene expression may be altered, for instance serving to reduce host defence responses (Ha et al. 2009; Wang et al. 2010; Ayer et al. 2014). This supports evidence of host immune attenuation or avoidance in the successful establishment of 
the symbiosis (Rodriguez-Lanetty et al. 2006a; Voolstra et al. 2009; Schnitzler and Weis 2010; Lehnert et al. 2014; Mohamed et al. 2016). At slightly higher ROS dosages, molecular signalling pathways may be activated, potentially contributed to by the translocation of signalling compounds from the symbiont (Chapter 4), which in turn will have a wide variety of molecular consequences (Richier et al. 2005; Lesser 2006, 2012). If antioxidant capacities are overwhelmed, high levels of ROS will damage DNA, proteins and membranes (Lesser 1997; Lesser 2006, 2011), and signal apoptosis/autophagic mechanisms (as is thought to contribute to symbiont expulsion in response to thermal- and light-induced stress (Lesser 2006; Weis 2008; Lesser 2011, 2012)), causing the breakdown of the novel symbiosis.

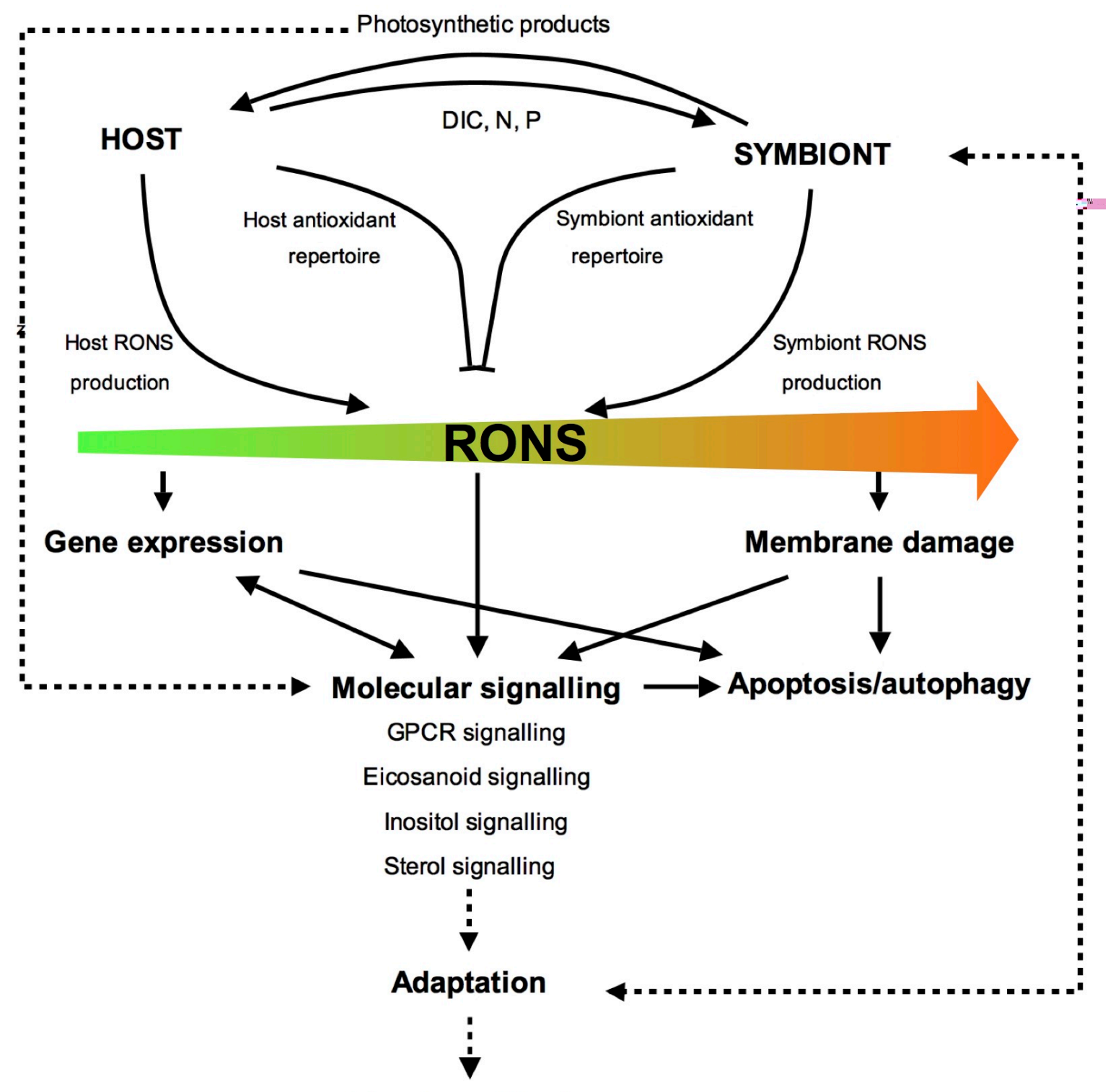

Novel symbiosis

Figure 6.1. Conceptual diagram for the molecular mechanisms behind the establishment of novel cnidarian-dinoflagellate symbioses. For detailed description see main text. RONS, reactive oxygen and nitrogen species. 
The findings from this thesis and the proposed conceptual model raise several important questions regarding the mechanisms that promote symbiosis flexibility: (1) If a disruption in oxidative homeostasis is a controlling factor for the molecular response to a novel symbiont, will the formation and persistence of evolutionary novelties in the symbiosis depend on the colonising symbionts' antioxidant repertoire? (2) How do symbiont-derived metabolites feature in the molecular signalling pathways of the host's cells and ultimately the success of the symbiosis? (3) What are the ecological implications of these findings for the survival of coral reefs? These questions will be considered further here.

\subsection{Mechanisms promoting flexibility}

\subsubsection{Will the formation of evolutionary novelties in the cnidarian-dinoflagellate} symbiosis depend on the symbionts' antioxidant repertoire?

RONS are produced through multiple mechanisms, depending on the cell and tissue types. In the cnidarian-dinoflagellate symbiosis, the host generates RONS at the cell membranes, mitochondria, peroxisomes and endoplasmic reticulum, while a major source of RONS is from chloroplasts of the photosynthesising-symbiont cell (Venn et al. 2008). RONS have many conserved functions, such as ROS- and NO- signalling and apoptosis, but the cellular response depends on the dose of RONS experienced. In order to allow for the opportunity for the symbiosis to adapt, RONS may need to be maintained at low levels, and the dosage of ROS may depend on the detoxifying capacity of the symbiont, host, or both (Figure 6.1). The balance between RONS production and removal in the symbiosis (redox homeostasis) is tightly controlled by the expression of a wide diversity of antioxidant mechanisms in both partners (Dykens and Shick 1982; Furla et al. 2005; Richier et al. 2005; Bou-Abdallah et al. 2006; Palmer et al. 2009; McGinty et al. 2012; Krueger et al. 2014; Krueger et al. 2015). Enzymatic antioxidants include ROS-scavenging superoxide dismutases (SODs), catalases and peroxidases, or disulphide reduction (e.g. thioredoxin, glutaredoxin), as well as non-enzymatic antioxidant molecules such as ascorbate, glutathione and uric acid (Dunlap and Yamamoto 1995; Dunlap et al. 1998; Meyer and Weis 2012; Krueger et al. 2015). The genus Symbiodinium has a diverse enzymatic antioxidant repertoire, and Symbiodinium type-specific isoforms have been described (Krueger et al. 2015). Indeed, peroxidase phylogenies have distinguished separate Symbiodinium clades, even to the point of resolving sub-cladal diversity between the Symbiodinium types C1 and C3 (Krueger et al. 2015). Furthermore, non-enzymatic antioxidants can be translocated from the symbiont to the host, and the abundance and diversity 
of these differ between symbiont types (Chapter 4). Some Symbiodinium types are considered more tolerant to oxidative stress than others (Wietheger et al. 2015), and may be linked to differential antioxidant responses (Roberty et al. 2016). It is therefore possible that the differential activity of the antioxidants will allow for some Symbiodinium types to be better equipped than others at reducing RONS toxicity when forming novel symbioses. A similar role for antioxidants in providing microbial-specific competency is observed in the establishment of other mutualisms. For instance, microbe-contaminated food induces an oxidative burst controlled by the $\mathrm{H}_{2} \mathrm{O}_{2}$-producing dual oxidase (DUOX) in the midgut of Drosophila melanogaster (Ha et al. 2005a), and host defence strongly depends on an immune-regulated catalase (Ha et al. 2005b). However, DUOX is negatively regulated in response to colonisation by resident bacteria (Ha et al. 2009), suggesting that ROS regulation plays an important role in the tolerance of resident bacteria and elimination of pathogenic ones. Furthermore, RONS play a role in the selection of the symbiotic partner in the bobtail squid (Davidson et al. 2004). Selection of the luminescent bacterium Vibrio fischeri in the colonisation of the light organ of the squid, while excluding non-symbiotic bacteria (the winnowing process; Nyholm and McFall-Ngai 2004) is partly linked to the chemical properties of the mucus coating the light organ epithelium (Davidson et al. 2004). Nitric oxide (NO) levels dictate the aggregation of bacteria in the epithelial mucus, and only competent bacteria producing specific NOdetoxifying catalase and luciferase enzymes, especially a flavohaemoglobin, allow them to colonise the squid (Wang et al. 2010).

Some Symbiodinium types are considered as 'generalists', able to associate with a large variety of cnidarian hosts, while conversely some symbionts have a smaller range of competent hosts and are considered as 'specialists' (Fabina et al. 2012). Host transmission mode is strongly correlated with symbiont specificity (i.e. generalist or specialist), with horizontallytransmitting corals being more likely to associate with generalist symbionts. Symbiodinium trenchii (ITS2 type D1a) used in this thesis is considered a 'generalist', and is known to associate with a wide range of coral species, and has recently been shown to have the ability to form novel associations via horizontal transmission in adult corals (Boulotte et al. 2016). D1a is commonly thought to provide increased thermal and oxidative resistance to cnidarians during periods of increased thermal and light stress (Berkelmans and Van Oppen 2006; Stat and Gates 2011). One mechanism believed to provide the increased oxidative (and thermal) stress-tolerance is the antioxidant repertoire of the symbiont (McGinty et al. 2012; Krueger et al. 2014; Wietheger et al. 2015). As mentioned earlier, the collapse of the symbiosis and establishment of novel associations evidently involves similar molecular responses (Chapter 
3). It stands to reason, therefore, that the oxidative stress-tolerance capacity of D1a and other oxidative stress-tolerant generalists may provide the fundamental prerequisite for establishing a novel association. In support of this, the generalist Symbiodinium type A1 possesses a higher oxidative stress tolerance relative to some other symbiont types, and demonstrated an investment of energetic resources into antioxidant production (Wietheger 2012; Wietheger et al. 2015). Symbiodinium type B1 may also be considered a generalist due to its symbiosis with a wide variety of cnidarian hosts (LaJeunesse 2002; Finney et al. 2010), and is also considered moderately robust to oxidative stress (Wietheger 2012; Wietheger et al. 2015). The temperate Symbiodinium californium in clade E produces higher levels of ROS and antioxidants relative to other types (Wietheger 2012; Wietheger et al. 2015), and although it is not thought to colonise scleractinian corals, clade E (S. voratum; Jeong et al 2014) was on occasion able to colonise at higher cell densities in Aiptasia sp. than its homologous type B1 (Starzak et al. 2014; but see Xiang et al. 2013). Further investigations into the expression, activity and abundance of antioxidant isoforms relative to the symbiosis specificity of individual Symbiodinium types would provide intriguing insights into why some Symbiodinium are generalists, and some specialists.

\subsubsection{How do symbiont-derived metabolites feature in the molecular signalling pathways of the host's cells?}

Intercellular molecular signals regulate many biochemical processes involved in the symbiosis, including host-symbiont recognition and phagocytosis, induction of host cell immunity and symbiont tolerance, metabolic exchange, and symbiosis dysfunction and breakdown (Weis 2008; Davy et al. 2012; Rosic et al. 2015). Transcriptomic analyses of symbiotic versus aposymbiotic gene expression in numerous anemone systems (Aiptasia sp., Anthopleura elegantissima, and Anemonia viridis) have revealed multiple potential molecular signalling proteins and mechanisms involved in the establishment, maintenance and breakdown of the symbiosis (Rodriguez-Lanetty et al. 2006a; Sunagawa et al. 2009; Ganot et al. 2011; Moya et al. 2012; Lehnert et al. 2014). Some of these proteins could be localised to the gastrodermis of the host (where the symbionts are contained in host-derived symbiosomes). Subsequently, research has begun to shed light on the function of some of the differentially-expressed gastrodermal genes in the hopes of identifying symbiosome-specific transporters (Dani et al. 2014). An example is the immunolocalisation experiments of Niemann-Pick type C (NPC1 and NPC2) immune regulation and sterol-trafficking proteins by Dani et al (2014). Although the exact process is unclear, the authors revealed an important role for NPC proteins in sterol transport and immune regulation in the stability and dysfunction of the cnidarian-dinoflagellate 
symbiosis. This comes from prior knowledge of NPC proteins in other biological systems. For example, NPC1 is well characterised in humans because of its role in the Niemann-Pick type C disease, which is a neurovisceral lipid storage disorder. Impairment of NPC1 disrupts intracellular lipid transport and results in the accumulation of cholesterol and glycosphingolipids in late endosomal/lysosomal compartments (Scott and Ioannou, 2004; Infante et al. 2008 a,b; Vance 2010). Additionally, several studies indicate that the NPC1 gene has a crucial role in binding cell-surface viral glyco-proteins of the Ebola virus in entry to human cells (Carette et al. 2011; Côté et al. 2011). The localisation of the NPC1 protein to the symbiosome could therefore be consistent with NPC1 being involved in the recognition of the symbiont cell-surface glycome (Logan et al. 2010), as well as participating in sterol trafficking from the algal symbionts to the cnidarian host. This is consistent with the upregulation of AvNPC2-d activity detected in Anemonia viridis in a stable symbiotic state (Dani et al. 2014). Differential expression of a NPC2 variant was observed in response to symbiotic state in Aiptasia sp. in Chapter 3. Interestingly, however, the expression patterns indicated a downregulation in the NPC2 variant in the symbiotic versus aposymbiotic state, which is contrary to previous studies in Aiptasia sp. (Lehnert et al. 2014; Oakley et al. 2016). Furthermore, while AvNPC2-d was downregulated during stress in A. viridis (Dani et al. 2014), NPC2 gene expression was higher in Aiptasia sp. in the heterologous association compared to the homologous association (Chapter 3). Meanwhile, no evidence for symbiontto-host translocation of sterol compounds was observed in stable isotope analysis under photosynthetic conditions, in any of the symbiotic states in Aiptasia sp. (Chapter 4). These results correlate with the absence of stable isotope labelled sterols in previous metabolite profiling studies in Aiptasia sp. (Hillyer 2016). Nevertheless, stable isotope analysis in Acropora aspera did reveal labelled pools of campesterol in the host tissues (Hillyer 2016), and there is also transcriptomic evidence for NPC1 protein expression in Anemonia viridis during symbiosis establishment (Dani et al. 2014), and NPC1 expression was upregulated during the day in Acropora millepora (Bertucci et al. 2015). Considered together, these results indicate a role for the NPC1/NPC2 system in sterol trafficking in cnidarians, although the response may not be homogeneous across cnidarians or environments.

Inositol derivatives are found in both the dinoflagellate symbiont (Klueter et al. 2015) and cnidarian host (Burriesci et al. 2012), and stable isotope analysis revealed large differences in the translocation of inositol and inositol phosphate isoforms between the symbiotic states (Chapter 4). This is consistent with previous studies that have identified inositol in the repertoire of photosynthetic products translocated from symbiont to host, in both Aiptasia sp. 
and corals (Acropora aspera) (Hillyer 2016). Along with functions as compatible solutes, inositol derivatives have highly-conserved functions in cellular recognition, signalling and development (Garrett et al. 2013; Rosic et al. 2015), and may function in the transduction of symbiosis dysfunction and cnidarian bleaching (Hillyer 2016). Inositol occurs in several different stereoisomers, of which the myo-form is the most abundant (Loewus and Dickinson 1982). It is used as a building-block of a cellular 'language' that plays various roles in signal transduction. Both lipids and phosphates can be added to myo-inositol, and then removed (Mitchell 2011), resulting in the production of new intracellular secondary messenger molecules that impart specific information to the cell (Gillaspy 2011). chiro-inositol is correlated with stress tolerance in plants (Ishitani et al., 1996; Nelson et al., 1998), while scyllo-inositol acts as an excellent carbon source in the nitrogen-fixing rhizobacterium Sinorhizobium meliloti, that forms a symbiosis with legumes (Kohler et al. 2010). Interestingly, scyllo-inositol was the isoform labelled irrespective of symbiont type, while myo-inositol was only labelled in a homologous association (Chapter 4). In addition, the relative abundance of labelled scyllo-inositol was much higher in homologous host tissues (Chapter 4). Inositol isoforms were found to be the most important predictor metabolites of temperature and light intensity across four Symbiodinium types (S. microadriaticum (type A1), S. minutum (type B1), S. psygmophilum (type B2) and S. trenchii (type D1a) (Klueter et al. 2015)). Myo-inositol concentration was found to be highest in B1 (Klueter et al. 2015), and thermal stress of an Aiptasia-B1 symbiosis induced significant increases in both myo- and scyllo-inositol translocation from symbiont to host (Hillyer 2016). Most interestingly, labelled scyllo-inositol pools in the host increased by ca. 1300\% compared to non-thermally-stressed controls (Hillyer 2016). In Montipora capitata, neither host nor symbiont pools of myo- and scyllo-inositol were affected by the homologous Symbiodinium community composition (Chapter 5). Given their prevalence in the cnidarian-dinoflagellate symbiosis, and evidence for symbiont-specific translocation, increased abundance during thermal stress, and a metabolic role in other symbioses, inositol compounds are clearly an important symbiont-derived metabolite in the cnidarian-dinoflagellate symbiosis.

Furthermore, the symbiotic interaction and signalling between legumes and rhizobacteria is mediated via phosphatidylinositol-regulated endocytosis (Peleg-Grossman et al. 2007). Indeed, rhizobial mutants that are deficient in the catabolism of inositol are often less competitive for nodule formation in the rhizobacterial-legume symbiosis (Gillaspy 2011). Interestingly, phosphatidylinositol signalling pathways were upregulated in response to a novel symbiont (Chapter 3). Inositol phosphates play major roles in calcium-signalling pathways and the 
induction of encystment of dinoflagellates (Tsim et al. 1997), and are also thought to play a role in the activity of cell apoptosis and autophagy (Boehning et al. 2005; Chakraborty et al. 2008; Gillaspy 2011), both modes of cell death, which are elevated during thermal stress and play a role in coral bleaching (Dunn et al. 2004; Dunn et al. 2007; Weis 2008). Glycosylphosphatidylinositol (GPI) is important for cell recognition and detection of microbes by the host (Davy et al. 2012; Rosic et al. 2015), and a GPI-linked carbonic anhydrase, with potential roles in DIC translocation to the symbiont, was downregulated in the heterologous host (Chapter 4). Inositol signalling pathways may therefore play an important role in the establishment, maintenance and breakdown of the symbiosis (Figure 6.2).

Despite the intriguing potential for inositol signalling pathways in the coral-dinoflagellate symbiosis, little research has been directed specifically at these compounds. Given the potential role for inositol signal transduction in coral bleaching and the evidence presented here that it may be involved in symbiosis specificity, targeted research on inositol signalling is a priority in the field. Conclusive evidence for the role of inositol translocation and signalling may come from: 1) In vitro studies with Symbiodinium mutants in which the genes involved in inositol metabolism, transport and signalling are silenced, followed by an evaluation of the capacity of these Symbiodinium cells to establish symbiosis with the host (however, we are still a long way from achieving this); 2) targeted metabolite profiling of inositol compounds in response to different biotic and abiotic factors; and/or 3) experimental supplementation/removal of inositol followed by evaluation of the symbiosis in response to different biotic and abiotic stressors. 


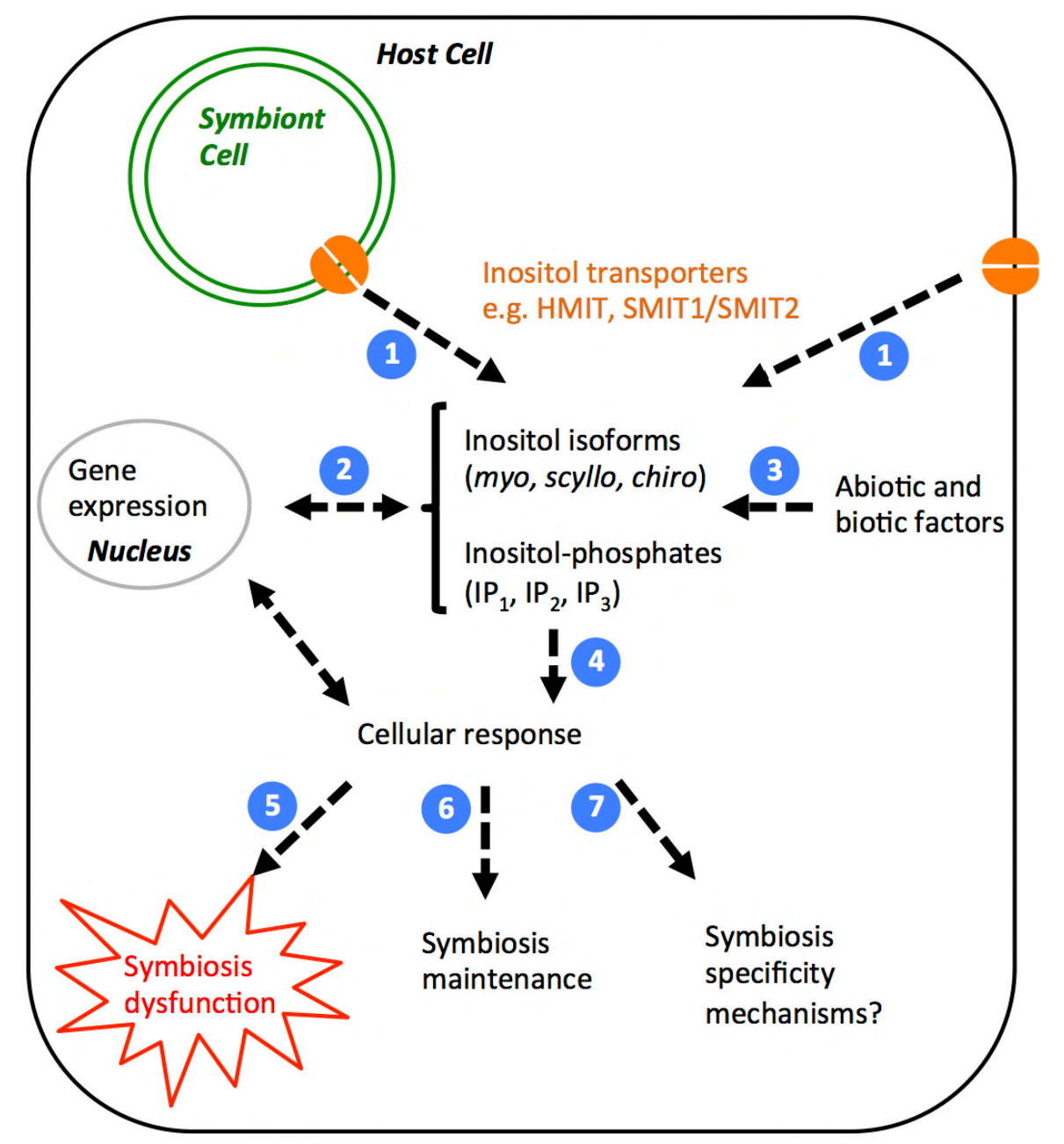

Figure 6.2 Potential inositol-signalling pathways. (1) Sugar/inositol co-transporters import sugars and inositol into the cells. (2) Inositol isoforms may then combine with phosphate groups to affect the gene expression of the cell. (3) Abiotic factors such as thermal stress, and biotic factors such as symbiont type may influence the abundance and form of inositol pools in the cell. (4) Depending on the inositol compound, a variety of cellular responses may result. (5) Inositol causes symbiosis dysfunction by activating $\mathrm{Ca}^{2+}$ influx that causes apoptotic and autophagic mechanisms. (6) Alternatively, inositol may act as a major carbon source for the cell, or maintain cellular signalling pathways that allow the tolerance of a symbiont. (7) However, the incorrect inositol isoform and/or abundance of inositol compounds may feature in symbiosis specificity mechanisms by activating specific phosphatidylinositol-signalling pathways.

\subsection{Implications for the survival of coral reefs}

Climate change is likely to be an important driver for changes in species composition and population structure on coral reefs. Current trends indicate the loss of stress-sensitive corals, and replacement by stress-tolerant corals able to survive the ecological disturbance. For instance, at the high-latitude reef of Sesoko Island (Okinawa, Japan), stress-sensitive branching pocilloporid corals were replaced by stress-tolerant massive corals such as poritids and brain corals following two mass bleaching events (in 1998 and 2001) (Van Woesik et al. 2011). The 
capacity to associate with different symbiont types (symbiotic flexibility) may afford these stress-sensitive corals the opportunity to withstand environmental fluctuations over time (Buddemeier and Fautin 1993; Fautin and Buddemeier 2004). My results with Aiptasia sp. demonstrate that potential adaptive mechanisms for symbiont flexibility in cnidarians entail the adaptation of optimal nutritional exchange and redox homeostasis (Chapters 3 and 4). Furthermore, symbiotic corals may possess compensatory mechanisms that allow for the persistence of an association with more thermotolerant/less nutritionally beneficial Symbiodinium types (Chapter 5). These adaptive mechanisms may be more prevalent than previously thought; for instance, until recently, the de novo acquisition of Symbiodinium types from the environment had not been demonstrated in corals, however the advent of improved molecular resolutions may show a greater potential for symbiotic flexibility and mechanisms of environmental acclimatisation in corals (Boulotte et al. 2016).

Mechanisms of host tolerance to environmental stressors are likely to influence the survival and distribution of coral communities (Wicks et al. 2011), but may also play a role in the ability of corals to change their symbionts. Indeed, studies of altered bleaching patterns over recent years suggest that at least some coral populations are adapting to contemporary warming (Maynard et al. 2008; Guest et al. 2012; Pratchett et al. 2013), and these adaptations may extend to allow corals to form functional symbioses with different Symbiodinium types. Host innate immune responses, apoptotic processes to remove cells damaged by photo- and temperature-related damage, and antioxidant and ROS removal strategies feature in the host's response to bleaching (Weis 2008; Baird et al. 2009a). Whether the result of differential mortality of stress-susceptible coral hosts (Sampayo et al. 2016), or due to genetic adaptation of the host (Howells et al. 2016; Jin et al. 2016), environmental variables will have cumulative effects on coral tolerance and resistance mechanisms (Nordemar et al. 2003; Schlöder and D'Croz 2004; Wooldridge 2009; Wicks et al. 2011). In light of the findings described in this thesis, that similar mechanisms are likely to be involved in the thermo- and oxidative-stress bleaching response and symbiosis specificity (Chapters 3 and 4; Figure 6.1), frequent exposure to thermal/oxidative stress may result in modification of host stress tolerance or resistance mechanisms, diminishing symbiosis-specificity processes and allowing for successful colonisation by novel symbiont types. In support of this, Boulotte et al. (2016) recently detected the acquisition of novel Symbiodinium types in the corals Pocillopora damicornis and Stylophora pistillata following consecutive bleaching events. Of significance, these corals employ vertical symbiont transmission as a life history trait (Baird et al. 2009b; Stat et al. 2015), and therefore show that these symbiotic associations are more flexible than 
previously thought (Boulotte et al. 2016), which has important implications for the prediction of coral assemblage changes following recovery from mass bleaching events. However, this hypothesis is contrary to evidence that corals that are predominantly sensitive to environmental disturbance display high flexibility in terms of the Symbiodinium species they can associate with (Putnam et al. 2012). For instance, corals that are able to form flexible associations with multiple Symbiodinium assemblages such as Acropora spp. are some of the most environmentally sensitive corals, while comparatively resistant corals such as Porites spp. exhibit low flexibility in Symbiodinium composition. Nevertheless, strategies by both partners are likely to influence the capacity for a novel symbiosis to evolve, and given the increasing frequency with which thermal stress anomalies are occurring, how temperature anomalies influence host stress tolerance mechanisms, and whether this will drive mechanisms enabling changes within the Symbiodinium community, is worthy of future investigation.

My results suggest that maintenance of a novel association may be restricted to those species that are able to withstand prolonged metabolic fluctuations, can adapt to a new nutrient regime, and/or possess a diverse antioxidant repertoire (Chapter 3-5). Current evidence suggests that more stress-tolerant Symbiodinium species, such as members of clades A and D, are typically less nutritionally beneficial ('parasitic') (Berkelmans and Van Oppen 2006; Jones et al. 2008; Jones and Berkelmans 2010; Jones and Berkelmans 2011; Stat and Gates 2011; Lesser et al. 2013). However, the nutritional contribution of the symbiont may not be of much concern if the coral is able to shift to more heterotrophic mechanisms to support its metabolic requirements (Hughes and Grottoli 2013). Tropical corals can obtain up to $70 \%$ of their own nutrients via heterotrophy (Houlbreque and Ferrier - Pagès 2009), and increased heterotrophy is observed in corals in response to decreased water clarity (Anthony and Fabricius 2000), decreased algal photosynthesis (Hoogenboom et al. 2010), or during thermal stress (Grottoli et al. 2006; Baumann et al. 2014). This compensatory mechanism may provide the opportunity for the maintenance and persistence of novel symbioses (Chapter 5). Furthermore, nutritional costs associated with clade D are lost in warmer waters (Cunning et al. 2015a), and so the increasing frequency of sea surface temperature anomalies may mean that nutritional limitations become less important, and thermal tolerance more important. The newly acquired symbionts may subsequently undergo a range of scenarios that will shape the future species composition or the reef; 1) the novel symbiont may constitute part of the background symbiont community (rare biosphere), providing the ability for corals to rapidly shuffle their symbionts in response to future warming events (Boulotte et al. 2016); 2) rapid symbiont adaptation via genetic recombination of Symbiodinium (e.g. Wilkinson et al. 2015) to reduce the pressure on 
heterotrophic compensatory mechanisms; 3) differential mortality of coral colonies that did not form the novel symbiosis will result in the prevalence of the novel association (Sampayo et al. 2016), or 4) hosts may select members of the novel symbiont population that are more beneficial than the others, resulting in a shift toward a more mutualistic state over time (Sachs and Wilcox 2006).

The effects of global climate change will not be homogeneous over space or time. Indeed, many coral reefs have formed with an immense diversity of cnidarian-dinoflagellate combinations, and the symbiont community can reflect spatial and temporal differences (Baker 2003; Santos et al. 2009; Stat et al. 2013). This is because biotic and abiotic factors influence the balance of symbiotic interaction costs and benefits (LaJeunesse et al. 2009; Cunning and Baker 2014; Cunning et al. 2015c). In light of evidence presented here, symbiotic flexibility is likely to become more prevalent on coral reefs, either as a result of increased environmental fluctuations providing the opportunity for selection or adaptation of flexible associations, or differential mortality of specific associations. This study provides a step change in our understanding the functional adaptation as to how cnidarians may form novel associations and survive climate change.

\subsection{Priorities for future work.}

This thesis provides a number of methodological advances that can be used in future studies to determine the molecular and metabolic implications of biotic and abiotic factors. Chapter 2 describes a simple and effective method of producing aposymbiotic anemones using menthol, which will rapidly provide large stocks of experimental organisms to study the cnidarian system and symbiosis. This method was adapted from a protocol optimised for the coral Stylophora pistillata (Wang et al. 2012). However the ability to colonise scleractinian coral hosts with different symbiont types was not examined here, or in the study by Wang et al. (2012). Given that the coral, symbiont and combinations of the two partners are likely to influence a variety of molecular responses, applying and optimising this approach to provide aposymbiotic scleractinian corals that can be re-colonised with a variety of Symbiodinium types should be a priority for future research.

Combining different levels of the 'omics cascade to study complex systems in an untargeted way provides novel insights into the underlying molecular pathways (Chapter 3). 'Omics studies, including genomics, transcriptomics, proteomics and metabolomics, have profoundly advanced our understanding of the cnidarian-dinoflagellate symbiosis in terms of the 
underlying molecules and their interactions (Gordon and Leggat 2010; Meyer and Weis 2012). However, lists of genes, proteins or metabolites only provide a partial or incomplete catalogue of the system, while a pathway-level analysis provides an improved understanding of the underlying biology and mechanistic aspects of the system (Chaston and Douglas 2012; Cavill et al. 2015). Statistically integrating parallel transcriptomic, proteomic and metabolomic data sets improves the sensitivity and confidence of pathway associations (Kamburov et al. 2011). Simultaneously, it is a hypothesis-driving technique, highlighting a number of molecular processes and candidate biomarkers within molecular processes that can be targeted in future studies (Cavill et al. 2015). To the best of my knowledge, integrating transcriptomic and metabolomic analysis has never been applied to a symbiotic system before, and here has provided an unbiased, non-targeted approach to study the underlying mechanisms of symbiosis specificity in the cnidarian-dinoflagellate symbiosis, as well as key biomarkers for future investigations. Applying untargeted integrated 'omics to other host-symbiont combinations, and under various climate change scenarios will further contribute to our understanding of this complex symbiosis. Improving the accessibility of current transcriptome and metabolite profiling data sets will facilitate these efforts.

Here, I focused on the heterologous symbiont S. trenchii (referred to in the study by its ITS2 type D1a), because it frequently populates corals that have recovered from bleaching (Stat and Gates 2011; Kemp et al. 2014; Boulotte et al. 2016). This has been attributed to its ability to associate with a variety of coral hosts and its relative thermal tolerance, although this is not a ubiquitous response (Thornhill et al. 2009; Stat and Gates 2011). The potential of D1a to provide resilience and assist in host recovery makes it an especially interesting candidate for investigations into the molecular and physiological bases of symbiosis (Berkelmans and Van Oppen 2006; Stat and Gates 2011). Conducting similar experiments with a variety of Symbiodinium types representing a range of colonisation strategies (generalists versus specialists), thermal sensitivities, and nutrient and antioxidant repertoires, will provide interesting comparisons to this study. Similarly, only the host tissues were examined in Chapters 3 and 4 , due to the inability to acquire sufficient symbiont biomass for symbiont fraction analysis; comparable data from the symbiont would, however, better enable a wholesystem understanding of the mechanisms involved. This will allow us to address some interesting questions that have arisen from this work, such as whether different Symbiodinium types express a different abundance and isoform variety of antioxidants and inositol, and whether any of the transporter expression profiles align with those of the host, indicating potential symbiosome-localised transporters. 


\subsection{Conclusions}

This thesis is novel in its application of integrated transcriptomics and metabolomics analyses, providing a greater understanding of the cellular processes involved in the cnidarian dinoflagellate symbiosis. Specifically, I identified the molecular processes and pathway activities affected in the host tissues of a model symbiosis (Aiptasia sp.) when colonised with different Symbiodinium types. This work demonstrated that colonisation with a novel symbiont type produces a gene and metabolite expression pattern intermediate between associating with the homologous symbiont and no symbiont at all. Pathway activity illustrated that an increase in the metabolism of stored energy, disruption of oxidative homeostasis, and molecular signalling processes were involved in colonisation by a novel Symbiodinium type. Subsequently, stable isotope labelling $\left({ }^{13} \mathrm{C}\right)$ GC-MS analysis provided evidence that the identity and relative abundance of photosynthetically-fixed carbon compounds contributed to host pools varies in response to different symbiont types. Finally, the intracellular metabolite pools of each symbiotic partner (both symbiont and host) in a coral (Montipora capitata) that naturally harbours genotypically different symbiont populations were compared. In this case, where all of these different naturally occurring symbiont types can be considered as 'homologous', there were no differences in the metabolite profiles of the corals or symbiotic dinoflagellates between the different coral-dinoflagellate associations. The combined results allowed for the development of a conceptual model to explain the potential onset and establishment of novel cnidarian-dinoflagellate symbioses. This model centres on optimal nutritional exchange and regulation of the oxidative state. The methodology described will facilitate future investigations into the regulation and collapse of the cnidarian-dinoflagellate symbiosis.

Given the potential for flexibility in the symbiosis to provide ecological benefits to coral hosts, understanding the cellular and molecular mechanisms driving symbiosis specificity is essential if we are to understand how coral reefs are likely to respond to climate change, and will also help to refine evolutionary models that predict the future of coral reefs. 


\section{REFERENCES}

Abrego D, Van Oppen MJH, Willis BL (2009a) Onset of algal endosymbiont specificity varies among closely related species of Acropora corals during early ontogeny. Molecular Ecology 18:3532-3543

Abrego D, Van Oppen MJH, Willis BL (2009b) Highly infectious symbiont dominates initial uptake in coral juveniles. Molecular Ecology 18:3518-3531

Ackman RG, Tocher C, McLachlan J (1968) Marine phytoplankter fatty acids. Journal of the Fisheries Board of Canada 25:1603-1620

Al-Moghrabi S, Goiran C, Allemand D, Speziale N, Jaubert J (1996) Inorganic carbon uptake for photosynthesis by the symbiotic coral-dinoflagellate association II. Mechanisms for bicarbonate uptake. Journal of Experimental Marine Biology and Ecology 199:227-248

Alexander RM (1964) Visco-elastic properties of the mesogloea of jellyfish. Journal of Experimental Biology 41:363-369

Allemand D, Tambutté É, Zoccola D, Tambutté S (2011) Coral calcification, cells to reefs. In Coral reefs: an ecosystem in transition. Springer Netherlands, pp119-150

Allwood JW, Heald J, Lloyd AJ, Goodacre R, Mur LA (2012) Separating the inseparable: the metabolomic analysis of plant-pathogen interactions. Methods in Molecular Biology 860:31-49

Altschul SF, Gish W, Miller W, Myers EW, Lipman DJ (1990) Basic local alignment search tool. Journal of Molecular Biology 215:403-410

Amores-Sánchez MI, Medina MÁ (1999) Glutamine, as a precursor of glutathione, and oxidative stress. Molecular Genetics and Metabolism 67:100-105

Anthony KR, Fabricius KE (2000) Shifting roles of heterotrophy and autotrophy in coral energetics under varying turbidity. Journal of Experimental Marine Biology and Ecology 252:221-253

Aranda M, DeSalvo MK, Bayer T, Medina M, Voolstra CR (2012) Evolutionary insights into scleractinian corals using comparative genomic hybridizations. BMC Genomics 13:501

Arif C, Daniels C, Bayer T, Banguera - Hinestroza E, Barbrook A, Howe CJ, LaJeunesse TC, Voolstra CR (2014) Assessing Symbiodinium diversity in scleractinian corals via next - generation sequencing - based genotyping of the ITS2 rDNA region. Molecular Ecology 23:4418-4433

Armstrong FAJ, Stearns CR, Strickland JDH (1967) The measurement of upwelling and subsequent biological process by means of the Technicon Autoanalyzer ${ }^{\circledR}$ and associated equipment. Deep Sea Research and Oceanographic Abstracts 14:381-389

Ayer A, Gourlay CW, Dawes IW (2014) Cellular redox homeostasis, reactive oxygen species and replicative ageing in Saccharomyces cerevisiae. FEMS Yeast Research 14:60-72

Bachar A, Achituv Y, Pasternak Z, Dubinsky Z (2007) Autotrophy versus heterotrophy: the origin of carbon determines its fate in a symbiotic sea anemone. Journal of Experimental Marine Biology and Ecology 349:295-298

Baghdasarian G, Muscatine L (2000) Preferential expulsion of dividing algal cells as a mechanism for regulating algal-cnidarian symbiosis. The Biological Bulletin 199:278-286

Baillie BK, Belda-Baillie CA, Silvestre V, Sison M, Gomez AV, Gomez ED, Monje V (2000) Genetic variation in Symbiodinium isolates from giant clams based on random-amplified-polymorphic DNA (RAPD) patterns. Marine Biology 136:829-836

Baird AH, Cumbo VR, Leggat W, Rodriguez-Lanetty M (2007) Fidelity and flexibility in coral symbioses. Marine Ecology Progress Series 347:307-309

Baird AH, Bhagooli R, Ralph PJ, Takahashi S (2009a) Coral bleaching: the role of the host. Trends in Ecology \& Evolution 24:16-20

Baird AH, Guest JR, Willis BL (2009b) Systematic and biogeographical patterns in the reproductive biology of scleractinian corals. Annual Reviews in Ecology and Evolution 40:551-571

Baker AC (2001) Ecosystems: reef corals bleach to survive change. Nature 411:765-766

Baker AC (2003) Flexibility and specificity in coral-algal symbiosis: Diversity, ecology, and biogeography of Symbiodinium. Annual Review of Ecology, Evolution, and Systematics 34:661-689

Baker AC, Starger CJ, McClanahan TR, Glynn PW (2004) Coral reefs: corals' adaptive response to climate change. Nature 430:741-741

Baker DM, Andras JP, Jordán-Garza AG, Fogel ML (2013) Nitrate competition in a coral symbiosis varies with temperature among Symbiodinium clades. The ISME Journal 7:1248-1251

Banaszak AT, Trench RK (1995) Effects of ultraviolet (UV) radiation on marine microalgal-invertebrate symbioses. II. The synthesis of mycosporine-like amino acids in response to exposure to UV in Anthopleura elegantissima and Cassiopeia xamachana. Journal of Experimental Marine Biology and Ecology 194:233-250

Banaszak AT, LaJeunesse TC, Trench RK (2000) The synthesis of mycosporine-like amino acids (MAAs) by cultured, symbiotic dinoflagellates. Journal of Experimental Marine Biology and Ecology 249:219-233 
Baran R, Reindl W, Northen TR (2009) Mass spectrometry based metabolomics and enzymatic assays for functional genomics. Current Opinion in Microbiology 12:547-552

Barneah O, Benayahu Y, Weis VM (2006) Comparative proteomics of symbiotic and aposymbiotic juvenile soft corals. Marine Biotechnology 8:11-16

Barott KL, Venn AA, Perez SO, Tambutté S, Tresguerres M (2015) Coral host cells acidify symbiotic algal microenvironment to promote photosynthesis. Proceedings of the National Academy of Sciences 112:607612

Barshis DJ, Stillman JH, Gates RD, Toonen RJ, Smith LW, Birkeland C (2010) Protein expression and genetic structure of the coral Porites lobata in an environmentally extreme Samoan back reef: does host genotype limit phenotypic plasticity? Molecular Ecology 19:1705-1720

Barshis DJ, Ladner JT, Oliver TA, Seneca FO, Traylor-Knowles N, Palumbi SR (2013) Genomic basis for coral resilience to climate change. Proceedings of the National Academy of Sciences of the United States of America 110:1387-1392

Barshis DJ, Ladner JT, Oliver TA, Palumbi SR (2014) Lineage-specific transcriptional profiles of Symbiodinium spp. unaltered by heat stress in a coral host. Molecular Biology and Evolution 31:1343-1352

Battey JF, Patton JS (1984) A reevaluation of the role of glycerol in carbon translocation in zooxanthellaecoelenterate symbiosis. Marine Biology 79:27-38

Baumann J, Grottoli AG, Hughes AD, Matsui Y (2014) Photoautotrophic and heterotrophic carbon in bleached and non-bleached coral lipid acquisition and storage. Journal of Experimental Marine Biology and Ecology 461:469-478

Baumgarten S, Simakov O, Esherick LY, Liew YJ, Lehnert EM, Michell CT, Li Y, Hambleton EA, Guse A, Oates M E (2015) The genome of Aiptasia, a sea anemone model for coral symbiosis. Proceedings of the National Academy of Sciences 112:11893-11898

Bay LK, Cumbo VR, Abrego D, Kool JT, Ainsworth TD, Willis BL (2011) Infection dynamics vary between Symbiodinium types and cell surface treatments during establishment of endosymbiosis with coral larvae. Diversity 3:356-374

Bayer T, Aranda M, Sunagawa S, Yum LK, Desalvo MK, Lindquist E, Coffroth MA, Voolstra CR, Medina M (2012) Symbiodinium transcriptomes: genome insights into the dinoflagellate symbionts of reef-building corals. PloS ONE 7:e35269

Belda-Baillie CA, Baillie BK, Maruyama T (2002) Specificity of a model cnidarian-dinoflagellate symbiosis. The Biological Bulletin 202:74-85

Bellantuono AJ, Hoegh-Guldberg O, Rodriguez-Lanetty M (2011) Resistance to thermal stress in corals without changes in symbiont composition. Proceedings of the Royal Society B: Biological Sciences 279:11001107

Berkelmans R, Van Oppen MJH (2006) The role of zooxanthellae in the thermal tolerance of corals: a 'nugget of hope' for coral reefs in an era of climate change. Proceedings of the Royal Society of London B: Biological Sciences 273:2305-2312

Bertucci A, Tambutté S, Supuran CT, Allemand D, Zoccola D (2011) A new coral carbonic anhydrase in Stylophora pistillata. Marine Biotechnology 13:992-1002

Bertucci A, Moya A, Tambutté S, Allemand D, Supuran CT, Zoccola D (2013) Carbonic anhydrases in anthozoan corals-A review. Bioorganic \& Medicinal Chemistry 21:1437-1450

Bertucci A, Foret S, Ball E, Miller DJ (2015) Transcriptomic differences between day and night in Acropora millepora provide new insights into metabolite exchange and light-enhanced calcification in corals. Molecular Ecology 24:4489-4504

Bidwell RGS (1967) Photosynthesis and metabolism in marine algae: VII. Products of photosynthesis in fronds of Fucus vesiculosus and their use in respiration. Canadian Journal of Botany 45:1557-1565

Bil KY, Kolmakov P, Parnik T, Titlyanov E, Muskatine L (1991) Photosynthetic products in zooxanthellae of the symbiotic corals Stylophora pistillata and Seriatopora coliendrum situated at various depths. Fiziol Rast (Moscow) 38:846-854

Bino RJ, Hall RD, Fiehn O, Kopka J, Saito K, Draper J, Nikolau BJ, Mendes P, Roessner-Tunali U, Beale MH (2004) Potential of metabolomics as a functional genomics tool. Trends in Plant Science 9:418-425

Birkemeyer C, Luedemann A, Wagner C, Erban A, Kopka J (2005) Metabolome analysis: the potential of in vivo labeling with stable isotopes for metabolite profiling. Trends in Biotechnology 23:28-33

Bishop DG, Kenrick JR (1980) Fatty acid composition of symbiotic zooxanthellae in relation to their hosts. Lipids 15:799-804

Björkman K, Karl DM (1994) Bioavailability of inorganic and organic phosphorus compounds to natural assemblages of microorganisms in Hawaiian coastal waters. Marine Ecology Progress Series Oldendorf 111:265-273

Blackall LL, Wilson B, van Oppen MJH (2015) Coral — the world's most diverse symbiotic ecosystem. Molecular Ecology 24:5330-5347

Blank RJ, Huss VA (1989) DNA divergency and speciation in Symbiodinium (Dinophyceae). Plant Systematics and Evolution 163:153-163 
Boehning D, van Rossum DB, Patterson RL, Snyder SH (2005) A peptide inhibitor of cytochrome c/inositol 1, 4, 5-trisphosphate receptor binding blocks intrinsic and extrinsic cell death pathways. Proceedings of the National Academy of Sciences of the United States of America 102:1466-1471

Bongaerts P, Riginos C, Ridgway T, Sampayo EM, van Oppen MJH, Englebert N, Vermeulen F, Hoegh-Guldberg O (2010) Genetic divergence across habitats in the widespread coral Seriatopora hystrix and its associated Symbiodinium. PloS ONE 5:e10871

Bou-Abdallah F, Chasteen ND, Lesser MP (2006) Quenching of superoxide radicals by green fluorescent protein. Biochimica et Biophysica Acta (BBA)-General Subjects 1760:1690-1695

Boulotte NM, Dalton SJ, Carroll AG, Harrison PL, Putnam HM, Peplow LM, van Oppen MJH (2016) Exploring the Symbiodinium rare biosphere provides evidence for symbiont switching in reef-building corals. The ISME Journal. 10:2693-2701

Bradford MM (1976) A rapid and sensitive method for the quantitation of microgram quantities of protein utilizing the principle of protein-dye binding. Analytical Biochemistry 72:248-254

Brading P, Warner ME, Davey P, Smith DJ, Achterberg EP, Suggett DJ (2011) Differential effects of ocean acidification on growth and photosynthesis among phylotypes of Symbiodinium (Dinophyceae). Limnology and Oceanography 56:927-938

Brading P, Warner ME, Smith DJ, Suggett DJ (2013) Contrasting modes of inorganic carbon acquisition amongst Symbiodinium (Dinophyceae) phylotypes. New Phytologist 200:432-442

Brown MR, Jeffrey S (1992) Biochemical composition of microalgae from the green algal classes Chlorophyceae and Prasinophyceae. 1. Amino acids, sugars and pigments. Journal of Experimental Marine Biology and Ecology 161:91-113

Brown BE, Le Tissier MDA, Bythell JC (1995) Mechanisms of bleaching deduced from histological studies of reef corals sampled during a natural bleaching event. Marine Biology 122:655-663

Brown BE (1997) Coral bleaching: causes and consequences. Coral Reefs 16:S129-S138

Buddemeier RW, Fautin DG (1993) Coral bleaching as an adaptive mechanism. Bioscience 43:320-326

Bundy JG, Davey MP, Viant MR (2009) Environmental metabolomics: a critical review and future perspectives. Metabolomics 5:3-21

Burke L., Selig L., Spalding M. (2002) Reefs at risk in Southeast Asia. The World Resources Institute, Washington, DC, USA

Burriesci MS, Raab TK, Pringle JR (2012) Evidence that glucose is the major transferred metabolite in dinoflagellate-cnidarian symbiosis. Journal of Experimental Biology 215:3467-3477

Byler KA, Carmi-Veal M, Fine M, Goulet TL (2013) Multiple symbiont acquisition strategies as an adaptive mechanism in the coral Stylophora pistillata. PloS ONE 8:e59596

Campbell BJ, Yu L, Heidelberg JF, Kirchman DL (2011) Activity of abundant and rare bacteria in a coastal ocean. Proceedings of the National Academy of Sciences 108:12776-12781

Cantin NE, van Oppen MJH, Willis BL, Mieog JC, Negri AP (2009) Juvenile corals can acquire more carbon from high-performance algal symbionts. Coral Reefs 28:405-414

Carette JE, Raaben M, Wong AC, Herbert AS, Obernosterer G, Mulherkar N, Kuehne AI, Kranzusch PJ, Griffin AM, Ruthel G (2011) Ebola virus entry requires the cholesterol transporter Niemann-Pick C1. Nature 477:340-343

Carpenter KE, Abrar M, Aeby G, Aronson RB, Banks S, Bruckner A, Chiriboga A, Cortés J, Delbeek JC, DeVantier L (2008) One-third of reef-building corals face elevated extinction risk from climate change and local impacts. Science 321:560-563

Cavill R, Jennen D, Kleinjans J, Briedé JJ (2015) Transcriptomic and metabolomic data integration. Briefings in Bioinformatics 17: 891-901

Chakraborty A, Koldobskiy MA, Sixt KM, Juluri KR, Mustafa AK, Snowman AM, Van Rossum DB, Patterson RL, Snyder SH (2008) HSP90 regulates cell survival via inositol hexakisphosphate kinase-2. Proceedings of the National Academy of Sciences 105:1134-1139

Chaston J, Douglas AE (2012) Making the most of "omics" for symbiosis research. The Biological Bulletin 223:2129

Chen H-K, Song S-N, Wang L-H, Mayfield AB, Chen Y-J, Chen W-NU, Chen C-S (2015) A compartmental comparison of major lipid species in a coral-Symbiodinium endosymbiosis: Evidence that the coral host regulates lipogenesis of its cytosolic lipid bodies. PloS ONE 10:e0132519

Chen M-C, Cheng Y-M, Sung P-J, Kuo C-E, Fang L-S (2003) Molecular identification of Rab7 (ApRab7) in Aiptasia pulchella and its exclusion from phagosomes harboring zooxanthellae. Biochemical and Biophysical Research Communications 308:586-595

Chen M-C, Cheng Y-M, Hong M-C, Fang L-S (2004) Molecular cloning of Rab5 (ApRab5) in Aiptasia pulchella and its retention in phagosomes harboring live zooxanthellae. Biochemical and Biophysical Research Communications 324:1024-1033

Chen W-N, Kang H-J, Weis VM, Mayfield AB, Jiang P-L, Fang L-S, Chen C-S (2012) Diel rhythmicity of lipidbody formation in a coral-Symbiodinium endosymbiosis. Coral Reefs 31:521-534 
Coffroth MA, Santos SR, Goulet TL (2001) Early ontogenetic expression of specificity in a cnidarian-algal symbiosis. Marine Ecology Progress Series 222:85-96

Coffroth MA, Santos SR (2005) Genetic diversity of symbiotic dinoflagellates in the genus Symbiodinium. Protist 156:19-34

Coffroth MA, Poland DM, Petrou EL, Brazeau DA, Holmberg JC (2010) Environmental symbiont acquisition may not be the solution to warming seas for reef-building corals. PloS ONE 5:e13258

Colley NJ, Trench R (1983) Selectivity in phagocytosis and persistence of symbiotic algae by the scyphistoma stage of the jellyfish Cassiopeia xamachana. Proceedings of the Royal Society of London Series B, Biological Sciences 219:61-82

Cook CB (1985) Equilibrium populations and long-term stability of mutualistic algae and invertebrate hosts. In: The biology of mutualism: Ecology and Evolution pp 171-191

Cook CB, D'Elia CF, Muller-Parker G (1988) Host feeding and nutrient sufficiency for zooxanthellae in the sea anemone Aiptasia pallida. Marine Biology 98:253-262

Cook CB, Davy SK (2001) Are free amino acids responsible for the' host factor' effects on symbiotic zooxanthellae in extracts of host tissue? Hydrobiologia 461:71-78

Cooksey KE, Cooksey B (1972) Turnover of photosynthetically fixed carbon in reef corals. Marine Biology 15:289-292

Cooper TF, Lai M, Ulstrup KE, Saunders SM, Flematti GR, Radford B, van Oppen MJH (2011a) Symbiodinium genotypic and environmental controls on lipids in reef building corals. PloS ONE 6:e20434

Cooper TF, Ulstrup KE, Dandan SS, Heyward AJ, Kuhl M, Muirhead A, O'Leary RA, Ziersen BE, van Oppen MJH (2011b) Niche specialization of reef-building corals in the mesophotic zone: metabolic trade-offs between divergent Symbiodinium types. Proceedings Biological Sciences / The Royal Society 278:18401850

Côté M, Misasi J, Ren T, Bruchez A, Lee K, Filone CM, Hensley L, Li Q, Ory D, Chandran K (2011) Small molecule inhibitors reveal Niemann-Pick C1 is essential for Ebola virus infection. Nature 477:344-348

Creek DJ, Chokkathukalam A, Jankevics A, Burgess KE, Breitling R, Barrett MP (2012) Stable isotope-assisted metabolomics for network-wide metabolic pathway elucidation. Analytical Chemistry 84:8442-8447

Croft D, Mundo AF, Haw R, Milacic M, Weiser J, Wu G, Caudy M, Garapati P, Gillespie M, Kamdar MR (2014) The Reactome pathway knowledgebase. Nucleic Acids Research 42:D472-D477

Crossland CJ, Barnes DJ (1977) Nitrate assimilation enzymes from two hard corals, Acropora acuminata and Goniastrea australensis. Comparative Biochemistry and Physiology Part B: Comparative Biochemistry 57:151-157

Crossland CJ, Barnes DJ, Borowitzka MA (1980) Diurnal lipid and mucus production in the staghorn coral Acropora acuminata. Marine Biology 60:81-90

Cumbo VR, Baird AH, van Oppen MJH (2013) The promiscuous larvae: flexibility in the establishment of symbiosis in corals. Coral Reefs 32:111-120

Cunning R, Baker AC (2014) Not just who, but how many: the importance of partner abundance in reef coral symbioses. Frontiers in Microbiology 5: 400

Cunning R, Gillette P, Capo T, Galvez K, Baker AC (2015a) Growth tradeoffs associated with thermotolerant symbionts in the coral Pocillopora damicornis are lost in warmer oceans. Coral Reefs 34:155-160

Cunning R, Yost DM, Guarinello ML, Putnam HM, Gates RD (2015b) Variability of Symbiodinium communities in waters, sediments, and corals of thermally distinct reef pools in American Samoa. PloS ONE 10:e0145099

Cunning R, Vaughan N, Gillette P, Capo T, Mate J, Baker AC (2015c) Dynamic regulation of partner abundance mediates response of reef coral symbioses to environmental change. Ecology 96:1411-1420

Cunning R, Ritson-Williams R, Gates RD (2016) Patterns of bleaching and recovery of Montipora capitata in Kāne 'ohe Bay, Hawai 'i, USA. Marine Ecology Progress Series 551:131-139

D'Elia CF, Domotor SL, Webb KL (1983) Nutrient uptake kinetics of freshly isolated zooxanthellae. Marine Biology 75:157-167

Dani V, Ganot P, Priouzeau F, Furla P, Sabourault C (2014) Are Niemann - Pick type C proteins key players in cnidarian-dinoflagellate endosymbioses? Molecular Ecology 23:4527-4540

Dani V, Priouzeau F, Pagnotta S, Carette D, Laugier J-P, Sabourault C (2016) Thermal and menthol stress induce different cellular events during sea anemone bleaching. Symbiosis 69:1-18

David M, Dzamba M, Lister D, Ilie L, Brudno M (2011) SHRiMP2: sensitive yet practical short read mapping. Bioinformatics 27:1011-1012

Davidson SK, Koropatnick TA, Kossmehl R, Sycuro L, McFall - Ngai MJ (2004) NO means 'yes' in the squid - Vibrio symbiosis: nitric oxide (NO) during the initial stages of a beneficial association. Cellular Microbiology 6:1139-1151

Davies PS (1984) The role of zooxanthellae in the nutritional energy requirements of Pocillopora eydouxi. Coral Reefs 2:181-186

Davies PS (1991) Effect of daylight variations on the energy budgets of shallow-water corals. Marine Biology 108:137-144 
Davy SK, Lucas IA, Turner JR (1996) Carbon budgets in temperate anthozoan-dinoflagellate symbioses. Marine Biology 126:773-783

Davy SK, Turner JR, Lucas IA (1997a) The nature of temperate anthozoan-dinoflagellate symbioses. Proceedings of the 7th International Coral Reef Symposium 2:1307-1312

Davy SK, Lucas IA, Turner JR (1997b) Uptake and persistence of homologous and heterologous zooxanthellae in the temperate sea anemone Cereus pedunculatus (Pennant). The Biological Bulletin 192:208-216

Davy SK, Cook CB (2001) The relationship between nutritional status and carbon flux in the zooxanthellate sea anemone Aiptasia pallida. Marine Biology 139:999-1005

Davy SK, Allemand D, Weis VM (2012) Cell biology of cnidarian-dinoflagellate symbiosis. Microbiology and Molecular Biology Reviews 76:229-261

Day AA, Udvardi MK (1993) Metabolite exchange across symbiosome membranes. Symbiosis 48: 493-523

Day DA, Kaiser BN, Thomson R, Udvardi MK, Moreau S, Puppo A (2001) Nutrient transport across symbiotic membranes from legume nodules. Functional Plant Biology 28:669-676

Deboer ML, Krupp DA, Weis VM (2007) Proteomic and transcriptional analyses of coral larvae newly engaged in symbiosis with dinoflagellates. Comparative Biochemistry and Physiology Part D: Genomics Proteomics 2:63-73

DeSalvo MK, Sunagawa S, Voolstra CR, Medina M (2010a) Transcriptomic responses to heat stress and bleaching in the elkhorn coral Acropora palmata. Marine Ecology Progress Series 402:97-113

DeSalvo MK, Sunagawa S, Fisher PL, Voolstra CR, Iglesias-Prieto R, Medina M (2010b) Coral host transcriptomic states are correlated with Symbiodinium genotypes. Molecular ecology 19:1174-1186

Detournay O, Schnitzler CE, Poole A, Weis VM (2012) Regulation of cnidarian-dinoflagellate mutualisms: Evidence that activation of a host TGFbeta innate immune pathway promotes tolerance of the symbiont. Developmental and Comparative Immunology 38:525-537

Díaz-Almeyda E, Thomé PE, El Hafidi M, Iglesias-Prieto R (2011) Differential stability of photosynthetic membranes and fatty acid composition at elevated temperature in Symbiodinium. Coral Reefs 30:217-225

Douglas AE (1994) Symbiotic interactions. Oxford University Press Oxford

Downs CA, Kramarsky-Winter E, Martinez J, Kushmaro A, Woodley CM, Loya Y, Ostrander GK (2009) Symbiophagy as a cellular mechanism for coral bleaching. Autophagy 5:211-216

Downs CA, McDougall KE, Woodley CM, Fauth JE, Richmond RH, Kushmaro A, Gibb SW, Loya Y, Ostrander GK, Kramarsky-Winter E (2013) Heat-stress and light-stress induce different cellular pathologies in the symbiotic dinoflagellate during coral bleaching. PloS ONE 8:e77173

Dudler N, Miller DJ (1988) Characterization of two glutamate dehydrogenases from the symbiotic microalga Symbiodinium microadriaticum isolated from the coral Acropora formosa. Marine Biology 97:427-430

Duerden JE (1902) West Indian madreporarian polyps. US Government Printing Office

Dunlap WC, Yamamoto Y (1995) Small-molecule antioxidants in marine organisms: antioxidant activity of mycosporine-glycine. Comparative Biochemistry and Physiology Part B: Biochemistry and Molecular Biology 112:105-114

Dunlap WC, Yamamoto Y, Inoue M, Kashiba - Iwatsuki M, Yamaguchi M, Tomita K (1998) Uric acid photo oxidation assay: in vitro comparison of sunscreening agents. International Journal of Cosmetic Science 20:1-18

Dunn SR, Thomason J, Le Tissier M, Bythell J (2004) Heat stress induces different forms of cell death in sea anemones and their endosymbiotic algae depending on temperature and duration. Cell Death \& Differentiation 11:1213-1222

Dunn SR, Schnitzler CE, Weis VM (2007) Apoptosis and autophagy as mechanisms of dinoflagellate symbiont release during cnidarian bleaching: every which way you lose. Proceedings of the Royal Society of London B: Biological Sciences 274:3079-3085

Dunn SR, Weis VM (2009) Apoptosis as a post-phagocytic winnowing mechanism in a coral-dinoflagellate mutualism. Environmental Microbiology 11:268-276

Dunn SR, Thomas MC, Nette GW, Dove SG (2012) A lipidomic approach to understanding free fatty acid lipogenesis derived from dissolved inorganic carbon within cnidarian-dinoflagellate symbiosis. PloS ONE 7:e46801

Dunn SR, Bythell JC, Le Tissier MD, Burnett WJ, Thomason JC (2002) Programmed cell death and cell necrosis activity during hyperthermic stress-induced bleaching of the symbiotic sea anemone Aiptasia sp. Journal of Experimental Marine Biology and Ecology 272:29-53

Dunstan GA, Volkman JK, Jeffrey SW, Barrett SM (1992) Biochemical composition of microalgae from the green algal classes Chlorophyceae and Prasinophyceae. 2. Lipid classes and fatty acids. Journal of Experimental MarineBbiology and Ecology 161:115-134

Dykens JA, Shick JM (1982) Oxygen production by endosymbiotic algae controls superoxide dismutase activity in their animal host. Nature 297: 579-580

Edmunds PJ, Pochon X, Levitan DR, Yost DM, Belcaid M, Putnam HM, Gates RD (2014) Long-term changes in Symbiodinium communities in Orbicella annularis in St. John, US Virgin Islands. Marine Ecology Progress Series 506:129-144 
Fabina NS, Putnam HM, Franklin EC, Stat M, Gates RD (2012) Transmission mode predicts specificity and interaction patterns in coral-Symbiodinium networks. PloS ONE 7:e44970

Fabina NS, Putnam HM, Franklin EC, Stat M, Gates RD. (2013). Symbiotic specificity, association patterns, and function determine community responses to global changes: defining critical research areas for coralSymbiodinium symbioses. Global Change Biology 19: 3306-3316.

Fabricius KE, Mieog JC, Colin PL, Idip D, van Oppen MJH (2004) Identity and diversity of coral endosymbionts (zooxanthellae) from three Palauan reefs with contrasting bleaching, temperature and shading histories. Molecular Ecology 13:2445-2458

Falkowski PG, Dubinsky Z, Muscatine L, Porter JW (1984) Light and the bioenergetics of a symbiotic coral. Bioscience 34:705-709

Falkowski PG, Dubinsky Z, Muscatine L, McCloskey L (1993) Population control in symbiotic corals. Bioscience 43:606-611

Farnese FS, Menezes-Silva PE, Gusman GS, Oliveira JA (2016) When bad guys become good ones: The key Role of reactive oxygen species and nitric oxide in the plant responses to abiotic stress. Frontiers in Plant Science 7: 471

Fautin DG, Buddemeier RW (2004) Adaptive bleaching: a general phenomenon Coelenterate Biology 2003 530: $459-467$

Fay SA, Weber MX (2012) The occurrence of mixed infections of Symbiodinium (Dinoflagellata) within individual hosts. Journal of Phycology 48:1306-1316

Ferrier-Pagès C, Peirano A, Abbate M, Cocito S, Negri A, Rottier C, Riera P, Rodolfo-Metalpa R, Reynaud S (2011) Summer autotrophy and winter heterotrophy in the temperate symbiotic coral Cladocora caespitosa. Limnology and Oceanography 56:1429-1438

Ferrier - Pagès C, Godinot C, D'Angelo C, Wiedenmann J, Grover R (2016) Phosphorus metabolism of reef organisms with algal symbionts. Ecological Monographs 86:262-277

Fiehn O (2001) Combining genomics, metabolome analysis, and biochemical modelling to understand metabolic networks. Comparative and Functional Genomics 2:155-168

Finney JC, Pettay DT, Sampayo EM, Warner ME, Oxenford HA, LaJeunesse TC (2010) The relative significance of host-habitat, depth, and geography on the ecology, endemism, and speciation of coral endosymbionts in the genus Symbiodinium. Microbial Ecology 60:250-263

Fitt WK, Trench RK (1981) Spawning, development, and acquisition of zooxanthellae by Tridacna squamosa (Mollusca, Bivalvia). The Biological Bulletin 161:213-235

Fitt WK, Trench RK (1983) Endocytosis of the symbiotic dinoflagellate Symbiodinium microadriaticum Freudenthal by endodermal cells of the scyphistomae of Cassiopeia xamachana and resistance of the algae to host digestion. Journal of Cell Science 64:195-212

Fleury BG, Coll JC, Tentori E, Duquesne S, Figueiredo L (2000) Effect of nutrient enrichment on the complementary (secondary) metabolite composition of the soft coral Sarcophyton ehrenbergi (Cnidaria: Octocorallia: Alcyonaceae) of the Great Barrier Reef. Marine Biology 136:63-68

Flynn KJ, Jones KJ, Raine R, Richard J, Flynn K (1994) Use of intracellular amino-acid-analysis as an indicator of the physiological status of natural dinoflagellate populations. Marine Ecology Progress Series 103:175186

Frade PR, De Jongh F, Vermeulen F, Van Bleijswijk J, Bak, RPM. (2008) Variation in symbiont distribution between closely related coral species over large depth ranges. Molecular Ecology 17:691-703

Franklin EC, Stat M, Pochon X, Putnam HM, Gates RD (2012) GeoSymbio: a hybrid, cloud - based web application of global geospatial bioinformatics and ecoinformatics for Symbiodinium-host symbioses. Molecular Ecology Resources 12:369-373

Fransolet D, Roberty S, Plumier J-C (2012) Establishment of endosymbiosis: The case of cnidarians and Symbiodinium. Journal of Experimental Marine Biology and Ecology 420-421:1-7

Freudenthal HD (1962) Symbiodinium gen. nov. and Symbiodinium microadriaticum sp. nov., a Zooxanthella: Taxonomy, life cycle, and morphology. The Journal of Protozoology 9:45-52

Fujise L, Yamashita H, Suzuki G, Koike K (2013) Expulsion of zooxanthellae (Symbiodinium) from several species of scleractinian corals: comparison under non-stress conditions and thermal stress conditions. Journal of Coral Reef Studies 15:29-36

Furla P, Allemand D, Shick JM, Ferrier-Pagès C, Richier S, Plantivaux A, Merle P-L, Tambutté S (2005) The symbiotic anthozoan: a physiological chimera between alga and animal. Integrative and Comparative Biology 45:595-604

Ganot P, Moya A, Magnone V, Allemand D, Furla P, Sabourault C (2011) Adaptations to endosymbiosis in a cnidarian-dinoflagellate association: differential gene expression and specific gene duplications. PLoS Genetics 7:e1002187

Garrett TA, Schmeitzel JL, Klein JA, Hwang JJ, Schwarz JA (2013) Comparative lipid profiling of the cnidarian Aiptasia pallida and its dinoflagellate symbiont. PloS ONE 8:e57975

Gates RD, Baghdasarian G, Muscatine L (1992) Temperature stress causes host cell detachment in symbiotic cnidarians: implications for coral bleaching. The Biological Bulletin 182:324-332 
Gates RD, Hoegh-Guldberg O, McFall-Ngai MJ, Bil KY, Muscatine L (1995) Free amino acids exhibit anthozoan" host factor" activity: They induce the release of photosynthate from symbiotic dinoflagellates in vitro. Proceedings of the National Academy of Sciences 92:7430-7434

Gates RD, Bil KY, Muscatine L (1999) The influence of an anthozoan "host factor" on the physiology of a symbiotic dinoflagellate. Journal of Experimental Marine Biology and Ecology 232:241-259

Gibbin EM, Davy SK (2013) Intracellular pH of symbiotic dinoflagellates. Coral Reefs 32:859-863

Gillaspy GE (2011) The cellular language of myo - inositol signaling. New Phytologist 192:823-839

Glynn PW (1996) Coral reef bleaching: facts, hypotheses and implications. Global Change Biology 2:495-509

Glynn PW, Maté JL, Baker AC, Calderón MO (2001) Coral bleaching and mortality in Panama and Ecuador during the 1997-1998 El Niño-Southern Oscillation event: spatial/temporal patterns and comparisons with the 1982-1983 event. Bulletin of Marine Science 69:79-109

Godinot C, Ferrier-Pagès C, Grover R (2009) Control of phosphate uptake by zooxanthellae and host cells in the scleractinian coral Stylophora pistillata. Limnology and Oceanography 54:1627

Gómez-Cabrera MdC, Ortiz J, Loh W, Ward S, Hoegh-Guldberg O (2008) Acquisition of symbiotic dinoflagellates (Symbiodinium) by juveniles of the coral Acropora longicyathus. Coral Reefs 27:219-226

Gordon BR, Leggat W (2010) Symbiodinium-invertebrate symbioses and the role of metabolomics. Marine Drugs 8:2546-2568

Gordon BR, Leggat W, Motti CA (2013) Extraction protocol for nontargeted NMR and LC-MS metabolomicsbased analysis of hard coral and their algal symbionts. Metabolomics Tools for Natural Product Discovery: Methods and Protocols 1055:129-147

Goulet TL (2006) Most corals may not change their symbionts. Marine Ecology Progress Series 321:1-7

Goulet TL, Cook CB, Goulet D (2005) Effect of short-term exposure to elevated temperatures and light levels on photosynthesis of different host-symbiont combinations in the Aiptasia pallida/Symbiodinium symbiosis. Limnology and Oceanography 50:1490-1498

Grabherr MG, Haas BJ, Yassour M, Levin JZ, Thompson DA, Amit I, Adiconis X, Fan L, Raychowdhury R, Zeng Q (2011) Full-length transcriptome assembly from RNA-Seq data without a reference genome. Nature Biotechnology 29:644-652

Grant AJ, Rémond M, Hinde R (1998) Low molecular-weight factor from Plesiastrea versipora (Scleractinia) that modifies release and glycerol metabolism of isolated symbiotic algae. Marine Biology 130:553-557

Grant AJ, Rémond M, Withers K, Hinde R (2001) Inhibition of algal photosynthesis by a symbiotic coral. Hydrobiologia 461:63-69

Grant AJ, Trautman DA, Frankland S, Hinde R (2003) A symbiosome membrane is not required for the actions of two host signalling compounds regulating photosynthesis in symbiotic algae isolated from cnidarians. Comparative Biochemistry and Physiology Part A: Molecular \& Integrative Physiology 135:337-345

Grant AJ, Trautman DA, Menz I, Hinde R (2006a) Separation of two cell signalling molecules from a symbiotic sponge that modify algal carbon metabolism. Biochemical and Biophysical Research Communications 348:92-98

Grant AJ, Rémond M, Starke-Peterkovic T, Hinde R (2006b) A cell signal from the coral Plesiastrea versipora reduces starch synthesis in its symbiotic alga, Symbiodinium sp. Comparative Biochemistry and Physiology Part A: Molecular \& Integrative Physiology 144:458-463

Grasshoff K, Ehrhardt M, and Kremling K (1983) Methods of seawater analysis, second revised and extended edition. John Wiley \& Sons

Grasshoff K, Kremling K, and Ehrhardt M (2009) Methods of seawater analysis, third, completely revised and extended edition. John Wiley \& Sons

Grottoli AG, Rodrigues LJ, Juarez C (2004) Lipids and stable carbon isotopes in two species of Hawaiian corals, Porites compressa and Montipora verrucosa, following a bleaching event. Marine Biology 145:621-631

Grottoli AG, Rodrigues LJ, Palardy JE (2006) Heterotrophic plasticity and resilience in bleached corals. Nature 440:1186-1189

Grottoli AG, Rodrigues LJ (2011) Bleached Porites compressa and Montipora capitata corals catabolize $\delta 13 \mathrm{C}$ enriched lipids. Coral Reefs 30:687-692

Grover R, Maguer J-F, Allemand D, Ferrier-Pagès C (2006) Urea uptake by the scleractinian coral Stylophora pistillata. Journal of Experimental Marine Biology and Ecology 332:216-225

Grover R, Maguer J-F, Allemand D, Ferrier-Pagès C (2008) Uptake of dissolved free amino acids by the scleractinian coral Stylophora pistillata. Journal of Experimental Biology 211:860-865

Guest JR, Baird AH, Maynard JA, Muttaqin E, Edwards AJ, Campbell SJ, Yewdall K, Affendi YA, Chou LM (2012) Contrasting patterns of coral bleaching susceptibility in 2010 suggest an adaptive response to thermal stress. PloS ONE 7:e33353

Gusmão LC (2010) Systematics and evolution of sea anemones (Cnidaria: Actiniaria: Hormathiidae) symbiotic with hermit crabs. Molecular Phylogenetics and Evolution 56:868-877

Guy C, Kaplan F, Kopka J, Selbig J, Hincha DK (2008) Metabolomics of temperature stress. Physiologia Plantarum 132:220-235 
Ha E-M, Oh C-T, Bae YS, Lee W-J (2005a) A direct role for dual oxidase in Drosophila gut immunity. Science 310:847-850

Ha E-M, Oh C-T, Ryu J-H, Bae Y-S, Kang S-W, Jang I-H, Brey PT, Lee W-J (2005b) An antioxidant system required for host protection against gut infection in Drosophila. Developmental Cell 8:125-132

Ha E-M, Lee K-A, Seo YY, Kim S-H, Lim J-H, Oh B-H, Kim J, Lee W-J (2009) Coordination of multiple dual oxidase-regulatory pathways in responses to commensal and infectious microbes in Drosophila gut. Nature Immunology 10:949-957

Haeseler G, Maue D, Grosskreutz J, Bufler J, Nentwig B, Piepenbrock S, Dengler R, Leuwer M (2002) Voltagedependent block of neuronal and skeletal muscle sodium channels by thymol and menthol. European Journal of Anaesthesiology 19:571-579

Hagedorn M, Carter V, Zuchowicz N, Phillips M, Penfield C, Shamenek B, Vallen EA, Kleinhans FW, Peterson K, White M (2015) Trehalose is a chemical attractant in the establishment of coral symbiosis. PloS ONE 10:e 0117087

Hans M, Wilhelm M, Swandulla D (2012) Menthol suppresses nicotinic acetylcholine receptor functioning in sensory neurons via allosteric modulation. Chemical Senses 37:463-469

Harii S, Yasuda N, Rodriguez-Lanetty M, Irie T, Hidaka M (2009) Onset of symbiosis and distribution patterns of symbiotic dinoflagellates in the larvae of scleractinian corals. Marine Biology 156:1203-1212

Harland AD, Fixter LM, Davies PS, Anderson RA (1991) Distribution of lipids between the zooxanthellae and animal compartment in the symbiotic sea anemone Anemonia viridis: Wax esters, triglycerides and fatty acids. Marine Biology 110:13-19

Harland AD, Navarro JC, Davies PS, Fixter LM (1993) Lipids of some Caribbean and Red Sea corals: total lipid, wax esters, triglycerides and fatty acids. Marine Biology 117:113-117

Harland AD, Davies PS (1995) Symbiont photosynthesis increases both respiration and photosynthesis in the symbiotic sea anemone Anemonia viridis. Marine Biology 123:715-722

Hartle-Mougiou K, D'angelo C, Smith EG, Burt J, West P, Wiedenmann J (2012) Diversity of zooxanthellae from corals and sea anemones after long-term aquarium culture. Journal of the Marine Biological Association of the United Kingdom 92:687-691

Hawkins TD, Davy SK (2012) Nitric oxide production and tolerance differ among Symbiodinium types exposed to heat stress. Plant and Cell Physiology 53:1889-1898

Hawkins TD (2013) Bleaching of the Cnidarian-Dinoflagellate Symbiosis: Aspects of Innate Immunity and the Role of Nitric Oxide. PhD Thesis, Victoria University of Wellington

Hawkins TD, Bradley BJ, Davy SK (2013) Nitric oxide mediates coral bleaching through an apoptotic-like cell death pathway: evidence from a model sea anemone-dinoflagellate symbiosis. The FASEB Journal 27:4790-4798

Hawkins TD, Hagemeyer J, Warner ME (2016a) Temperature moderates the infectiousness of two conspecific Symbiodinium strains isolated from the same host population. Environmental Microbiology 18:5204-5217

Hawkins TD, Hagemeyer JC, Hoadley KD, Marsh AG, Warner ME (2016b) Partitioning of respiration in an animal-algal symbiosis: Implications for different aerobic capacity between Symbiodinium spp. Frontiers in physiology 7:128

Hayes RL, Goreau NI (1977) Intracellular crystal-bearing vesicles in the epidermis of scleractinian corals, Astrangia danae (Agassiz) and Porites porites (Pallas). The Biological Bulletin 152:26-40

Hemond EM, Vollmer SV (2015) Diurnal and nocturnal transcriptomic variation in the Caribbean staghorn coral, Acropora cervicornis. Molecular Ecology 24:4460-4473

Herre EA, Knowlton N, Mueller UG, Rehner SA (1999) The evolution of mutualisms: exploring the paths between conflict and cooperation. Trends in Ecology \& Evolution 14:49-53

Hill R, Fernance C, Wilkinson SP, Davy SK, Scott A (2014) Symbiont shuffling during thermal bleaching and recovery in the sea anemone Entacmaea quadricolor. Marine Biology 161:2931-2937

Hiller K, Hangebrauk J, Jäger C, Spura J, Schreiber K, Schomburg D (2009) MetaboliteDetector: comprehensive analysis tool for targeted and nontargeted GC/MS based metabolome analysis. Analytical Chemistry 81:3429-3439

Hiller K, Metallo CM, Kelleher JK, Stephanopoulos G (2010) Nontargeted elucidation of metabolic pathways using stable-isotope tracers and mass spectrometry. Analytical Chemistry 82:6621-6628

Hiller K, Wegner A, Weindl D, Cordes T, Metallo CM, Kelleher JK, Stephanopoulos G (2013) NTFD—a standalone application for the non-targeted detection of stable isotope-labeled compounds in GC/MS data. Bioinformatics 29:1226-1228

Hillyer KE (2016) Thermal Stress and Bleaching in the Cnidarian-Dinoflagellate Symbiosis: The Application of Metabolomics. PhD Thesis, Victoria University of Wellington

Hillyer KE, Tumanov S, Villas-Boas SG, Davy SK (2016a) Metabolite profiling of symbiont and host during thermal stress and bleaching in a model cnidarian-dinoflagellate symbiosis. Journal of Experimental Biology 219:516-527

Hillyer KE, Dias DA, Lutz A, Wilkinson SP, Roessner U, Davy SK (2016b) Metabolite profiling of symbiont and host during thermal stress and bleaching in the coral Acropora aspera. Coral Reefs 219:516-527 
Hines A, Oladiran GS, Bignell JP, Stentiford GD, Viant MR (2007) Direct sampling of organisms from the field and knowledge of their phenotype: key recommendations for environmental metabolomics. Environmental Science \& Technology 41:3375-3381

Hirai MY, Yano M, Goodenowe DB, Kanaya S, Kimura T, Awazuhara M, Arita M, Fujiwara T, Saito K (2004) Integration of transcriptomics and metabolomics for understanding of global responses to nutritional stresses in Arabidopsis thaliana. Proceedings of the National Academy of Sciences of the United States of America 101:10205-10210

Hirai MY, Klein M, Fujikawa Y, Yano M, Goodenowe DB, Yamazaki Y, Kanaya S, Nakamura Y, Kitayama M, Suzuki H (2005) Elucidation of gene-to-gene and metabolite-to-gene networks in Arabidopsis by integration of metabolomics and transcriptomics. Journal of Biological Chemistry 280:25590-25595

Hirose M, Reimer JD, Hidaka M, Suda S (2008) Phylogenetic analyses of potentially free-living Symbiodinium spp. isolated from coral reef sand in Okinawa, Japan. Marine Biology 155:105-112

Hoegh-Guldberg O (1999) Climate change, coral bleaching and the future of the world's coral reefs. Marine and Freshwater Research 50:839-866

Hoegh-Guldberg O, Mumby PJ, Hooten AJ, Steneck RS, Greenfield P, Gomez E, Harvell CD, Sale PF, Edwards AJ, Caldeira K (2007) Coral reefs under rapid climate change and ocean acidification. Science 318:17371742

Hoegh-Guldberg O, Ortiz JC, Dove S (2011) The future of coral reefs. Science 334:1494

Hoeksema BW (1991) Control of bleaching in mushroom coral populations (Scleractinia: Fungiidae) in the Java Sea: Stress tolerance and interference by life history strategy. Marine Ecology Progress series Oldendorf 74:225-237

Hoeksema BW, Matthews JL (2011) Contrasting bleaching patterns in mushroom coral assemblages at Koh Tao, Gulf of Thailand. Coral Reefs 30:95-95

Hohman TC, McNeil PL, Muscatine L (1982) Phagosome-lysosome fusion inhibited by algal symbionts of Hydra viridis. The Journal of Cell Biology 94:56-63

Hoogenboom M, Beraud E, Ferrier-Pagès C (2010) Relationship between symbiont density and photosynthetic carbon acquisition in the temperate coral Cladocora caespitosa. Coral Reefs 29:21-29

Hosoi K (1938) Contribution to the biochemistry of the coral. 1. On the occurrence of glycogen and its content in the polyp of Fungia actiniformis var palawensis Döderlein. Palao Tropical Biology Station 3:447-451

Houlbreque F, Ferrier - Pagès C (2009) Heterotrophy in tropical scleractinian corals. Biological Reviews 84:1-17

Howells EJ, Beltran VH, Larsen NW, Bay LK, Willis BL, van Oppen MJH (2012) Coral thermal tolerance shaped by local adaptation of photosymbionts. Nature Climate Change 2:116-120

Howells EJ, Abrego D, Meyer E, Kirk NL, Burt JA (2016) Host adaptation and unexpected symbiont partners enable reef - building corals to tolerate extreme temperatures. Global Change Biology 22:2702-2714

Hu Z-L, Bao J, Reecy JM (2008) CateGOrizer: a web-based program to batch analyze gene ontology classification categories. Online Journal of Bioinformatics 9:108-112

Hughes AD, Grottoli AG, Pease TK, Matsui Y (2010) Acquisition and assimilation of carbon in non-bleached and bleached corals. Marine Ecology Progress Series 420:91-101

Hughes AD, Grottoli AG (2013) Heterotrophic compensation: a possible mechanism for resilience of coral reefs to global warming or a sign of prolonged stress? PloS ONE 8:e81172

Hulpiau P, Driege Y, Staal J, Beyaert R (2016) MALT1 is not alone after all: identification of novel paracaspases. Cellular and Molecular Life Sciences 73:1103-1116

Hume B, D’angelo C, Burt J, Baker AC, Riegl B, Wiedenmann J (2013) Corals from the Persian/Arabian Gulf as models for thermotolerant reef-builders: prevalence of clade C3 Symbiodinium, host fluorescence and ex situ temperature tolerance. Marine Pollution Bulletin 72:313-322

Hunter RL, LaJeunesse TC, Santos SR (2007) Structure and evolution of the rDNA Internal Transcribed Spacer (ITS) region 2 in the symbiotic dinoflagellates (Symbiodinium, Dinophyta). Journal of Phycology 43:120128

Iglesias-Prieto R, Trench RK (1997) Acclimation and adaptation to irradiance in symbiotic dinoflagellates. II. Response of chlorophyll-protein complexes to different photon-flux densities. Marine Biology 130:23-33

Iglesias-Prieto R, Beltran VH, LaJeunesse TC, Reyes-Bonilla H, Thome PE (2004) Different algal symbionts explain the vertical distribution of dominant reef corals in the eastern Pacific. Proceedings of the Royal Society of London Series B-Biological Sciences 271:1757-1763

Imbs AB, Rodkina SA (2004) Isolation of 2-methyl branched unsaturated very long fatty acids from marine sponge Halichondria panicea and identification of them by GC-MS and NMR. Chemistry and Physics of Lipids 129:173-181

Imbs AB, Latyshev NA, Dautova TN, Latypov YY (2010) Distribution of lipids and fatty acids in corals by their taxonomic position and presence of zooxanthellae. Marine Ecology Progress Series 409:65-75

Imbs AB, Yakovleva IM (2012) Dynamics of lipid and fatty acid composition of shallow-water corals under thermal stress: an experimental approach. Coral Reefs 31:41-53 
Imbs AB, Yakovleva IM, Dautova TN, Bui LH, Jones P (2014) Diversity of fatty acid composition of symbiotic dinoflagellates in corals: evidence for the transfer of host PUFAs to the symbionts. Phytochemistry 101:76-82

Infante RE, Abi-Mosleh L, Radhakrishnan A, Dale JD, Brown MS, Goldstein JL (2008) Purified NPC1 protein I. Binding of cholesterol and oxysterols to a 1278-amino acid membrane protein. Journal of Biological Chemistry 283:1052-1063

Infante RE, Radhakrishnan A, Abi-Mosleh L, Kinch LN, Wang ML, Grishin NV, Goldstein JL, Brown MS (2008) Purified NPC1 Protein II. Localization of sterol binding to a 240-amino acid soluble luminal loop. Journal of Biological Chemistry 283:1064-1075

Isa Y, Yamazato K (1984) The distribution of carbonic anhydrase in a staghorn coral Acropora hebes (Dana). Galaxea 3:25-36

Jackson AE, Yellowlees D (1990) Phosphate uptake by zooxanthellae isolated from corals. Proceedings of the Royal Society of London Series B: Biological Sciences 242:201-204

Jennings DB, Ehrenshaft M, Pharr DM, Williamson JD (1998) Roles for mannitol and mannitol dehydrogenase in active oxygen-mediated plant defense. Proceedings of the National Academy of Sciences 95:15129-15133

Jeong, H.J., Yoo, Y.D., Kang, N.S., Lim, A.S., Seong, K.A., Lee, S.Y., Lee, M.J., Lee, K.H., Kim, H.S., Shin, W., Nam, S.W., Yih, W. \& Lee, K. (2012). Heterotrophic feeding as a newly identified survival strategy of the dinoflagellate Symbiodinium. Proceedings of the National Academy of Sciences USA, 109: 12604-12609.

Jiang P-L, Pasaribu B, Chen C-S (2014) Nitrogen-deprivation elevates lipid levels in Symbiodinium spp. by lipid droplet accumulation: morphological and compositional analyses. PloS ONE 9:e87416

Jimbo M, Yanohara T, Koike K, Koike K, Sakai R, Muramoto K, Kamiya H (2000) The d-galactose-binding lectin of the octocoral Sinularia lochmodes: characterization and possible relationship to the symbiotic dinoflagellates. Comparative Biochemistry and Physiology Part B: Biochemistry and Molecular Biology 125:227-236

Jimbo M, Yamashita H, Koike K, Sakai R, Kamiya H (2010) Effects of lectin in the scleractinian coral Ctenactis echinata on symbiotic zooxanthellae. Fisheries Science 76:355-363

Jimbo M, Suda Y, Koike K, Nakamura-Tsuruta S, Kominami J, Kamei M, Hirabayashi J, Sakai R, Kamiya H (2013) Possible involvement of glycolipids in lectin-mediated cellular transformation of symbiotic microalgae in corals. Journal of Experimental Marine Biology and Ecology 439:129-135

Jin YK, Lundgren P, Lutz A, Raina J-B, Howells EJ, Paley AS, Willis BL, van Oppen MJH (2016) Genetic markers for antioxidant capacity in a reef-building coral. Science Advances 2:e1500842

Jokiel PL, Brown EK (2004) Global warming, regional trends and inshore environmental conditions influence coral bleaching in Hawaii. Global Change Biology 10:1627-1641

Jolley E, Smith DC (1978) The green hydra symbiosis. New Phytologist 81:637-645

Jolley E, Smith DC (1980) The green hydra symbiosis. II. The biology of the establishment of the association. Proceedings of the Royal Society of London Series B Biological Sciences 207:311-333

Jones R, Hoegh - Guldberg O, Larkum A, Schreiber U (1998) Temperature - induced bleaching of corals begins with impairment of the $\mathrm{CO} 2$ fixation mechanism in zooxanthellae. Plant, Cell \& Environment 21:12191230

Jones AM, Berkelmans R, van Oppen MJH, Mieog JC, Sinclair W (2008) A community change in the algal endosymbionts of a scleractinian coral following a natural bleaching event: field evidence of acclimatization. Proceedings of the Royal Society B: Biological Sciences 275:1359-1365

Jones AM, Berkelmans R (2010) Potential costs of acclimatization to a warmer climate: growth of a reef coral with heat tolerant vs. sensitive symbiont types. PloS ONE 5:e10437

Jones AM, Berkelmans R (2011) Tradeoffs to thermal acclimation: Energetics and reproduction of a reef coral with heat tolerant Symbiodinium type-D. Journal of Marine Biology 2011:1-12

Jones AM, Berkelmans R (2012) The photokinetics of thermo-tolerance in Symbiodinium. Marine Ecology 33:490-498

Junker BH, Klukas C, Schreiber F (2006) VANTED: a system for advanced data analysis and visualization in the context of biological networks. BMC Bioinformatics 7:1

Kamburov A, Cavill R, Ebbels TM, Herwig R, Keun HC (2011) Integrated pathway-level analysis of transcriptomics and metabolomics data with IMPaLA. Bioinformatics 27:2917-2918

Kaniewska P, Campbell PR, Kline DI, Rodriguez-Lanetty M, Miller DJ, Dove S, Hoegh-Guldberg O (2012) Major cellular and physiological impacts of ocean acidification on a reef building coral. PloS ONE 7:e34659

Kellogg R, Patton J (1983) Lipid droplets, medium of energy exchange in the symbiotic anemone Condylactis gigantea: a model coral polyp. Marine Biology 75:137-149

Kemp DW, Hernandez-Pech X, Iglesias-Prieto R, Fitt WK, Schmidt GW (2014) Community dynamics and physiology of Symbiodinium spp. before, during, and after a coral bleaching event. Limnology and Oceanography 59:788-797

Kérouel R, Aminot A (1997) Fluorometric determination of ammonia in sea and estuarine waters by direct segmented flow analysis. Marine Chemistry 57:265-275

Kerr KF (2009) Comments on the analysis of unbalanced microarray data. Bioinformatics 25:2035-2041 
Kim CS, Lim WA, Cho YC (2001) Mycosporine-like amino acids as the UV sunscreen with oxygen radical scavenging activity. Bulletin of National Fisheries Research and Development Agency Korea 60:65-71

Kinzie RA (1974) Experimental infection of aposymbiotic gorgonian polyps with zooxanthellae. Journal of Experimental Marine Biology and Ecology 15:335-345

Kinzie RA, Chee GS (1979) The effect of different zooxanthellae on the growth of experimentally reinfected hosts. The Biological Bulletin 156:315-327

Klueter A, Crandall JB, Archer FI, Teece MA, Coffroth MA (2015) Taxonomic and environmental variation of metabolite profiles in marine dinoflagellates of the genus Symbiodinium. Metabolites 5:74-99

Kneeland J, Hughen K, Cervino J, Hauff B, Eglinton T (2013) Lipid biomarkers in Symbiodinium dinoflagellates: new indicators of thermal stress. Coral Reefs 32:923-934

Koga R, Tsuchida T, Fukatsu T (2003) Changing partners in an obligate symbiosis: a facultative endosymbiont can compensate for loss of the essential endosymbiont Buchnera in an aphid. Proceedings of the Royal Society of London Series B: Biological Sciences 270:2543-2550

Kohler PR, Zheng JY, Schoffers E, Rossbach S (2010) Inositol catabolism, a key pathway in Sinorhizobium meliloti for competitive host nodulation. Applied and environmental microbiology 76:7972-7980

Kolde R (2013) pheatmap: Pretty Heatmaps. R package version 7. 61

Kopp C, Pernice M, Domart-Coulon I, Djediat C, Spangenberg JE, Alexander DT, Hignette M, Meziane T, Meibom A (2013) Highly dynamic cellular-level response of symbiotic coral to a sudden increase in environmental nitrogen. mBio 4:e00052-00013

Kopp C, Wisztorski M, Revel J, Mehiri M, Dani V, Capron L, Carette D, Fournier I, Massi L, Mouajjah D (2014) MALDI-MS and NanoSIMS imaging techniques to study cnidarian-dinoflagellate symbioses. Zoology 118:125-131

Kopp C, Domart-Coulon I, Escrig S, Humbel BM, Hignette M, Meibom A (2015) Subcellular investigation of photosynthesis-driven carbon assimilation in the symbiotic reef coral Pocillopora damicornis. mBio 6:e02299-02214

Krueger T, Becker S, Pontasch S, Dove S, Hoegh - Guldberg O, Leggat W, Fisher PL, Davy SK (2014) Antioxidant plasticity and thermal sensitivity in four types of Symbiodinium sp. Journal of phycology 50:1035-1047

Krueger T, Fisher PL, Becker S, Pontasch S, Dove S, Hoegh-Guldberg O, Leggat W, Davy SK (2015) Transcriptomic characterization of the enzymatic antioxidants FeSOD, MnSOD, APX and KatG in the dinoflagellate genus Symbiodinium. BMC evolutionary biology 15:1

Krupp DA, Weis VM (2006) Two atypical carbonic anhydrase homologs from the planula larva of the scleractinian coral Fungia scutaria. The Biological Bulletin 211:18-30

Kuo J, Chen MC, Lin CH, Fang LS (2004) Comparative gene expression in the symbiotic and aposymbiotic Aiptasia pulchella by expressed sequence tag analysis. Biochemical and Biophysical Research Communications 318:176-186

Kuo J, Liang Z-C, Lin C-H (2010) Suppression subtractive hybridization identifies genes correlated to symbiotic and aposymbiotic sea anemone associated with dinoflagellate. Journal of Experimental Marine Biology and Ecology 388:11-19

Kvennefors EC, Leggat W, Hoegh-Guldberg O, Degnan BM, Barnes AC (2008) An ancient and variable mannosebinding lectin from the coral Acropora millepora binds both pathogens and symbionts. Developmental and Comparative Immunology 32:1582-1592

Ladner JT, Barshis DJ, Palumbi SR (2012) Protein evolution in two co-occurring types of Symbiodinium: an exploration into the genetic basis of thermal tolerance in Symbiodinium clade D. BMC Evolutionary Biology 12:217

LaJeunesse TC (2001) Investigating the biodiversity, ecology, and phylogeny of endosymbiotic dinoflagellates in the genus Symbiodinium using the ITS region: in search of a "species" level marker. Journal of Phycology 37:866-880

LaJeunesse TC (2002) Diversity and community structure of symbiotic dinoflagellates from Caribbean coral reefs. Marine Biology 141:387-400

LaJeunesse TC, Thornhill D, Cox E, Stanton F, Fitt W, Schmidt G (2004) High diversity and host specificity observed among symbiotic dinoflagellates in reef coral communities from Hawaii. Coral Reefs 23:596603

LaJeunesse TC (2005) "Species" radiations of symbiotic dinoflagellates in the Atlantic and Indo-Pacific since the Miocene-Pliocene transition. Molecular Biology and Evolution 22:570-581

LaJeunesse TC, Lee S, Bush S, Bruno JF (2005) Persistence of non-Caribbean algal symbionts in Indo-Pacific mushroom corals released to Jamaica 35 years ago. Coral Reefs 24:157-159

LaJeunesse TC, Bonilla HR, Warner M, Wills M, Schmidt G, Fitt W (2008) Specificity and stability in high latitude eastern Pacific coral-algal symbioses. Limnology and Oceanography 53:719

LaJeunesse TC, Smith RT, Finney J, Oxenford H (2009) Outbreak and persistence of opportunistic symbiotic dinoflagellates during the 2005 Caribbean mass coral 'bleaching'event. Proceedings of the Royal Society of London B: Biological Sciences 276:4139-4148 
LaJeunesse TC, Fitt W, Schmidt G (2010a) The reticulated chloroplasts of zooxanthellae (Symbiodinium) and differences in chlorophyll localization among life cycle stages. Coral Reefs 29:627-627

LaJeunesse TC, Pettay DT, Sampayo EM, Phongsuwan N, Brown B, Obura DO, Hoegh-Guldberg O, Fitt WK (2010b) Long-standing environmental conditions, geographic isolation and host-symbiont specificity influence the relative ecological dominance and genetic diversification of coral endosymbionts in the genus Symbiodinium. Journal of Biogeography 37:785-800

LaJeunesse TC, Smith R, Walther M, Pinzón J, Pettay DT, McGinley M, Aschaffenburg M, Medina-Rosas P, Cupul-Magaña AL, Pérez AL (2010c) Host-symbiont recombination versus natural selection in the response of coral-dinoflagellate symbioses to environmental disturbance. Proceedings of the Royal Society of London B: Biological Sciences 283:rspb20100385

LaJeunesse TC, Thornhill DJ (2011) Improved resolution of reef-coral endosymbiont (Symbiodinium) species diversity, ecology, and evolution through psbA non-coding region genotyping. PloS ONE 6:e29013

LaJeunesse TC, Parkinson JE, Reimer JD (2012) A genetics - based description of Symbiodinium minutum sp. nov. and S. psygmophilum sp. nov.(Dinophyceae), two dinoflagellates symbiotic with cnidaria. Journal of Phycology 48:1380-1391

LaJeunesse TC, Wham DC, Pettay DT, Parkinson JE, Keshavmurthy S, Chen CA (2014) Ecologically differentiated stress-tolerant endosymbionts in the dinoflagellate genus Symbiodinium (Dinophyceae) Clade D are different species. Phycologia 53:305-319

Lauretta D, Häussermann V, Brugler MR, Rodríguez E (2014) Isoparactis fionae sp. nov.(Cnidaria: Anthozoa: Actiniaria) from Southern Patagonia with a discussion of the family Isanthidae. Organisms Diversity \& Evolution 14:31-42

Leal MC, Nunes C, Kempf S, Reis A, Da Silva TL, Serôdio J, Cleary D,FR Calado R (2013) Effect of light, temperature and diet on the fatty acid profile of the tropical sea anemone Aiptasia pallida. Aquaculture Nutrition 19:818-826

Leal MC, Hoadley K, Pettay DT, Grajales A, Calado R, Warner ME (2015) Symbiont type influences trophic plasticity of a model cnidarian-dinoflagellate symbiosis. Journal of Experimental Biology 218:858-863

Leggat W, Marendy EM, Baillie B, Whitney SM, Ludwig M, Badger MR, Yellowlees D (2002) Dinoflagellate symbioses: strategies and adaptations for the acquisition and fixation of inorganic carbon. Functional Plant Biology 29:309-322

Leggat W, Yellowlees D, Medina M (2011a) Recent progress in Symbiodinium transcriptomics. Journal of Experimental Marine Biology and Ecology 408:120-125

Leggat W, Seneca F, Wasmund K, Ukani L, Yellowlees D, Ainsworth TD (2011b) Differential responses of the coral host and their algal symbiont to thermal stress. PloS ONE 6:e26687

Lehnert EM, Burriesci MS, Pringle JR (2012) Developing the anemone Aiptasia as a tractable model for cnidariandinoflagellate symbiosis: the transcriptome of aposymbiotic A. pallida. BMC Genomics 13:271

Lehnert EM, Mouchka ME, Burriesci MS, Gallo ND, Schwarz JA, Pringle JR (2014) Extensive differences in gene expression between symbiotic and aposymbiotic cnidarians. G3 4:277-295

Lesser M (1997) Oxidative stress causes coral bleaching during exposure to elevated temperatures. Coral Reefs 16:187-192

Lesser MP (2006) Oxidative stress in marine environments: biochemistry and physiological ecology. Annual Review of Physiology 68:253-278

Lesser MP (2011) Coral bleaching: causes and mechanisms In Coral reefs: an ecosystem in transition, pp405-419: Springer

Lesser MP (2012) Oxidative stress in tropical marine ecosystems. In Oxidative Stress in Aquatic Ecosystems pp919: Blackwell publishing

Lesser MP, Stat M, Gates RD (2013) The endosymbiotic dinoflagellates (Symbiodinium sp.) of corals are parasites and mutualists. Coral Reefs 32:603-611

Lewis DH, Smith DC (1971) The autotrophic nutrition of symbiotic marine coelenterates with special reference to hermatypic corals. I. Movement of photosynthetic products between the symbionts. Proceedings of the Royal Society of London Series B Biological Sciences 178:111-129

Lewis JB, Price WS (1975) Feeding mechanisms and feeding strategies of Atlantic reef corals. Journal of Zoology 176:527-544

Ley RE, Peterson DA, Gordon JI (2006) Ecological and evolutionary forces shaping microbial diversity in the human intestine. Cell 124:837-848

Li H-H, Huang Z-Y, Ye S-P, Lu C-Y, Cheng P-C, Chen S-H, Chen C-S (2014) Membrane labeling of coral gastrodermal cells by biotinylation: The proteomic identification of surface proteins involving cnidariadinoflagellate endosymbiosis. PloS ONE 9:e85119

Libro S, Kaluziak ST, Vollmer SV (2013) RNA-seq profiles of immune related genes in the staghorn coral Acropora cervicornis infected with white band disease. PloS ONE 8:e81821

Lin K-L, Wang J-T, Fang L-S (2000) Participation of glycoproteins on zooxanthellal cell walls in the establishment of a symbiotic relationship with the sea anemone, Aiptasia pulchella. Zoological Studies 39:172-178 
Little AF, van Oppen MJH, Willis BL (2004) Flexibility in algal endosymbioses shapes growth in reef corals. Science 304:1492-1494

Loescher WH, Tyson RH, Everard JD, Redgwell RJ, Bieleski RL (1992) Mannitol synthesis in higher plants evidence for the role and characterization of a NADPH-dependent mannose 6-phosphate reductase. Plant Physiology 98:1396-1402

Loewus FA, Dickinson DB (1982) Cyclitols Plant Carbohydrates I. In Plant Carbohydrates I: Intracellular Carbohydrates pp193-216: Springer

Logan DD, LaFlamme AC, Weis VM, Davy SK (2010) Flow-cytometric characterization of the cell - surface glycans of symbiotic dinoflagellates (Symbiodinium spp.) 1. Journal of Phycology 46:525-533

Logares R, Audic S, Bass D, Bittner L, Boutte C, Christen R, Claverie J-M, Decelle J, Dolan JR, Dunthorn M (2014) Patterns of rare and abundant marine microbial eukaryotes. Current Biology 24:813-821

Lõhelaid H, Teder T, Samel N (2015) Lipoxygenase-allene oxide synthase pathway in octocoral thermal stress response. Coral Reefs 34:143-154

Loram JE, Trapido-Rosenthal HG, Douglas AE (2007) Functional significance of genetically different symbiotic algae Symbiodinium in a coral reef symbiosis. Molecular Ecology 16:4849-4857

Love MI, Huber W, Anders S (2014) Moderated estimation of fold change and dispersion for RNA-seq data with DESeq2. Genome Biology 15:1

Magtanong L, Ko PJ, Dixon SJ (2016) Emerging roles for lipids in non-apoptotic cell death. Cell Death \& Differentiation 23:1099-1109

Manning MM, Gates RD (2008) Diversity in populations of free-living Symbiodinium from a Caribbean and Pacific reef. Limnology and Oceanography 53:1853

Margulis L (1973) Symbiosis and evolution. Readings in Biology and Man 225:48-57

Marlow HQ, Martindale MQ (2007) Embryonic development in two species of scleractinian coral embryos: Symbiodinium localization and mode of gastrulation. Evolution \& Development 9:355-367

Matthews JL, Sproles AE, Oakley CA, Grossman AR, Weis VM, Davy SK (2016) Menthol-induced bleaching rapidly and effectively provides experimental aposymbiotic sea anemones (Aiptasia sp.) for symbiosis investigations. Journal of Experimental Biology 219:306-310

May P, Wienkoop S, Kempa S, Usadel B, Christian N, Rupprecht J, Weiss J, Recuenco-Munoz L, Ebenhöh O, Weckwerth W (2008) Metabolomics-and proteomics-assisted genome annotation and analysis of the draft metabolic network of Chlamydomonas reinhardtii. Genetics 179:157-166

Maynard J, Anthony K, Marshall P, Masiri I (2008) Major bleaching events can lead to increased thermal tolerance in corals. Marine Biology 155:173-182

McAuley PJ, Smith DC (1982a) The green hydra symbiosis. V. Stages in the intracellular recognition of algal symbionts by digestive cells. Proceedings of the Royal Society of London Series B Biological Sciences 216:7-23

McAuley PJ, Smith DC (1982b) The green hydra symbiosis. VII. Conservation of the host cell habitat by the symbiotic algae. Proceedings of the Royal Society of London Series B Biological Sciences 216:415-426

McAuley PJ (1994) Amino acid content of zooxanthellae freshly isolated from Pocillopora damicornis. Pacific Science 48:247-253

McAuley PJ, Cook CB (1994) Effects of host feeding and dissolved ammonium on cell division and nitrogen status of zooxanthellae in the hydroid Myrionema amboinense. Marine Biology 121:343-348

McClanahan TR (2004) The relationship between bleaching and mortality of common corals. Marine Biology 144:1239-1245

McGinley MP, Aschaffenburg MD, Pettay DT, Smith RT, LaJeunesse TC, Warner ME (2012) Transcriptional response of two core photosystem genes in Symbiodinium spp. exposed to thermal stress. PloS ONE 7:e50439

McGinty ES, Pieczonka J, Mydlarz LD (2012) Variations in reactive oxygen release and antioxidant activity in multiple Symbiodinium types in response to elevated temperature. Microbial Ecology 64:1000-1007

McKemy DD, Neuhausser WM, Julius D (2002) Identification of a cold receptor reveals a general role for TRP channels in thermosensation. Nature 416:52-58

McNeil PL, Smith DC (1982) The green hydra symbiosis. IV: Entry of symbionts into digestive cells. Proceedings of the Royal Society of London Series B Biological Sciences 216:1-6

Merle P-L, Sabourault C, Richier S, Allemand D, Furla P (2007) Catalase characterization and implication in bleaching of a symbiotic sea anemone. Free Radical Biology and Medicine 42:236-246

Mews LK, Smith D (1982) The green hydra symbiosis. VI. What is the role of maltose transfer from alga to animal? Proceedings of the Royal Society of London Series B Biological Sciences 216:397-413

Meyer E, Aglyamova GV, Matz MV (2011) Profiling gene expression responses of coral larvae (Acropora millepora) to elevated temperature and settlement inducers using a novel RNA - Seq procedure. Molecular Ecology 20:3599-3616

Meyer E, Weis VM (2012) Study of cnidarian-algal symbiosis in the "omics" age. The Biological Bulletin 223:4465 
Mieog JC, Olsen JL, Berkelmans R, Bleuler-Martinez SA, Willis BL, van Oppen MJH (2009) The roles and interactions of symbiont, host and environment in defining coral fitness. PloS ONE 4:e6364

Milacic M, Haw R, Rothfels K, Wu G, Croft D, Hermjakob H, D'Eustachio P, Stein L (2012) Annotating cancer variants and anti-cancer therapeutics in reactome. Cancers 4:1180-1211

Miller DJ, Yellowlees D (1989) Inorganic nitrogen uptake by symbiotic marine cnidarians: a critical review. Proceedings of the Royal Society of London B Biological Sciences 237:109-125

Miralles J, Diop M, Ferrer A, Kornprobst J-M (1989) Fatty acid composition of five zoantharia from the senegalese coast: Palythoa Dartevellei pax, P. monodi pax, P. Variabilis duerden, P. Senegalensis pax and their associated zooxanthellae and $P$. senegambiensis carter and its commensal, the decapoda diogenes ovatus miers. Comparative Biochemistry and Physiology Part B: Comparative Biochemistry 94:91-97

Moberg F, Folke C (1999) Ecological goods and services of coral reef ecosystems. Ecological Economics 29:215233

Mohamed AR, Cumbo V, Harii S, Shinzato C, Chan CX, Ragan MA, Bourne DG, Willis BL, Ball EE, Satoh N (2016) The transcriptomic response of the coral Acropora digitifera to a competent Symbiodinium strain: the symbiosome as an arrested early phagosome. Molecular Ecology 25: 3127-3141

Moné Y, Monnin D, Kremer N (2014) The oxidative environment: a mediator of interspecies communication that drives symbiosis evolution. Proceedings of the Royal Society of London B: Biological Sciences 281:20133112

Montano S, Seveso D, Galli P, Obura DO (2010) Assessing coral bleaching and recovery with a colour reference card in Watamu Marine Park, Kenya. Hydrobiologia 655:99-108

Moya A, Tambutté S, Bertucci A, Tambutté E, Lotto S, Vullo D, Supuran CT, Allemand D, Zoccola D (2008) Carbonic anhydrase in the scleractinian coral Stylophora pistillata characterization, localization, and role in biomineralization. Journal of Biological Chemistry 283:25475-25484

Moya A, Ganot P, Furla P, Sabourault C (2012) The transcriptomic response to thermal stress is immediate, transient and potentiated by ultraviolet radiation in the sea anemone Anemonia viridis. Molecular Ecology 21:1158-1174

Muller - Parker G, Lee KW, Cook CB (1996) Changes in the ultrastructure of symbiotic zooxanthellae (Symbiodinium sp. Dinophyceae) in fed and starved sea anemones maintained under high and low light Journal of Phycology 32:987-994

Muller-Parker G, D’Elia CF (1997) Interactions between corals and their symbiotic algae. In: Birkeland, C. (Ed.), Life and death of coral reefs. Chapman and Hall, New York pp 96-113

Muller - Parker G, Davy SK (2001) Temperate and tropical algal-sea anemone symbioses. Invertebrate Biology 120:104-123

Murphy MP (2009) How mitochondria produce reactive oxygen species. Biochemical Journal 417:1-13

Murphy J, Riley JP (1962) A modified single solution method for the determination of phosphate in natural waters. Analytica Chimica Acta 27:31-36

Muscatine L, Hand C (1958) Direct evidence for the transfer of materials from symbiotic algae to the tissues of a coelenterate. Proceedings of the National Academy of Sciences of the United States of America 44:1259

Muscatine L (1965) Symbiosis of hydra and algae-III. Extracellular products of the algae. Comparative Biochemistry and Physiology 16:77-92

Muscatine L (1967) Glycerol excretion by symbiotic algae from corals and Tridacna and its control by the host. Science 156:516-519

Muscatine L, Karakashian SJ, Karakashian MW (1967) Soluble extracellular products of algae symbiotic with a ciliate, a sponge and a mutant Hydra. Comparative Biochemistry and Physiology 20:1-12

Muscatine L (1973) Nutrition of corals. Biology and Geology of Coral Reefs 2:77-115

Muscatine L (1990) The role of symbiotic algae in carbon and energy flux in reef corals. In: Dubinsky Z (ed) Ecosystems of the world: Coral reefs. Elsevier, Amsterdam, pp75 - 87

Muscatine L, Cernichiari E (1969) Assimilation of photosynthetic products of zooxanthellae by a reef coral. The Biological Bulletin 137:506-523

Muscatine L, Porter JW (1977) Reef corals: mutualistic symbioses adapted to nutrient-poor environments. Bioscience 27:454-460

Muscatine L, Pool RR (1979) Regulation of numbers of intracellular algae. Proceedings of the Royal Society of London Series B Biological Sciences 204:131-139

Muscatine L, Pool RR, Cernichiari E (1972) Some factors influencing selective release of soluble organic material by zooxanthellae from reef corals. Marine Biology 13:298-308

Muscatine L, Pool RR, Trench R (1975) Symbiosis of algae and invertebrates: aspects of the symbiont surface and the host-symbiont interface. Transactions of the American Microscopical Society 94:450-469

Muscatine L, McCloskey LR, Marian RE(1981) Estimating the daily contribution of carbon from zooxanthellae to coral animal respiration. Oceanography 26:601-611

Muscatine L, Falkowski PG, Dubinsky Z (1983) Carbon budgets in symbiotic associations. In Schenk, H.E.A and W. Schwemmler (Eds.) Endocytobiology II. Intracellular spaces as oligogenetic system. Walter de Gruyter \& co., Berlin, New York. pp649-658 
Muscatine L, Falkowski PG, Porter JW, Dubinsky Z (1984) Fate of photosynthetic fixed carbon in light-and shadeadapted colonies of the symbiotic coral Stylophora pistillata. Proceedings of the Royal Society of London Series B Biological Sciences 222:181-202

Muscatine L, Porter JW, Kaplan IR (1989a) Resource partitioning by reef corals as determined from stable isotope composition. Marine Biology 100:185-193

Muscatine L, Falkowski PG, Dubinsky Z, Cook PA, McCloskey LH (1989b) The effect of external nutrient resources on the population dynamics of zooxanthellae in a reef coral. Proceedings of the Royal Society of London B: Biological Sciences 236:311-324

Muscatine L, Grossman D, Doino J (1991) Release of symbiotic algae by tropical sea anemones and corals after cold shock. Marine Ecological Progress Series 77:233-243

Muscatine L, Goiran C, Land L, Jaubert J, Cuif J-P, Allemand D (2005) Stable isotopes $\left(\delta^{13} \mathrm{C}\right.$ and $\left.\delta^{15} \mathrm{~N}\right)$ of organic matrix from coral skeleton. Proceedings of the National Academy of Sciences of the United States of America 102:1525-1530

Nitschke MR, Davy SK, Cribb TH, Ward S (2014) The effect of elevated temperature and substrate on free-living Symbiodinium cultures. Coral Reefs 34:1-11

Nordemar I, Nyström M, Dizon R (2003) Effects of elevated seawater temperature and nitrate enrichment on the branching coral Porites cylindrica in the absence of particulate food. Marine Biology 142:669-677

Nyholm SV, McFall-Ngai M (2004) The winnowing: establishing the squid-Vibrio symbiosis. Nature Reviews Microbiology 2:632-642

Oakley CA, Schmidt GW, Hopkinson BM (2014) Thermal responses of Symbiodinium photosynthetic carbon assimilation. Coral Reefs 33:501-512

Oakley CA, Ameismeier MF, Peng L, Weis VM, Grossman AR, Davy SK (2016) Symbiosis induces widespread changes in the proteome of the model cnidarian Aiptasia. Cellular Microbiology 18:1009-1023

Obata T, Schoenefeld S, Krahnert I, Bergmann S, Scheffel A, Fernie AR (2013) Gas-chromatography massspectrometry (GC-MS) based metabolite profiling reveals mannitol as a major storage carbohydrate in the coccolithophorid alga Emiliania huxleyi. Metabolites 3:168-184

Okazawa M, Terauchi T, Shiraki T, Matsumura K, Kobayashi S (2000) 1 - Menthol - induced [Ca2+] i increase and impulses in cultured sensory neurons. Neuroreport 11:2151-2155

Oku H, Yamashiro H, Onaga K (2003a) Lipid biosynthesis from [14C] - glucose in the coral Montipora digitata. Fisheries Science 69:625-631

Oku H, Yamashiro H, Onaga K, Sakai K, Iwasaki H (2003b) Seasonal changes in the content and composition of lipids in the coral Goniastrea aspera. Coral Reefs 22:83-85

Oliver TA, Palumbi SR (2010) Many corals host thermally resistant symbionts in high-temperature habitat. Coral Reefs 30:241-250

Ortiz JC, González - Rivero M, Mumby PJ (2013) Can a thermally tolerant symbiont improve the future of Caribbean coral reefs? Global change biology 19:273-281

Ortiz JC, González-Rivero M, Mumby PJ (2014) An ecosystem-level perspective on the host and symbiont traits needed to mitigate climate change impacts on Caribbean coral reefs. Ecosystems 17:1-13

Ott BM, Cruciger M, Dacks AM, Rio RV (2014) Hitchhiking of host biology by beneficial symbionts enhances transmission. Scientific reports 4:5825

Padilla-Gamiño JL, Pochon X, Bird C, Concepcion GT, Gates RD (2012) From parent to gamete: vertical transmission of Symbiodinium (Dinophyceae) ITS2 sequence assemblages in the reef building coral Montipora capitata. PloS ONE 7:e38440

Palmer CV, Modi CK, Mydlarz LD (2009) Coral fluorescent proteins as antioxidants. PloS ONE 4:e7298

Pang ZP, Südhof TC (2010) Cell biology of Ca 2+-triggered exocytosis. Current Opinion in Cell Biology 22:496505

Papina M, Meziane T, van Woesik R (2003) Symbiotic zooxanthellae provide the host-coral Montipora digitata with polyunsaturated fatty acids. Comparative Biochemistry and Physiology Part B: Biochemistry and Molecular Biology 135:533-537

Parkinson JE, Banaszak AT, Altman NS, LaJeunesse TC, \& Baums IB (2015). Intraspecific diversity among partners drives functional variation in coral symbioses. Scientific reports 5:15667.

Parkinson JE, Baumgarten S, Michell CT, Baums IB, LaJeunesse TC, Voolstra CR (2016) Gene expression variation resolves species and individual strains among coral-associated dinoflagellates within the genus Symbiodinium. Genome Biology and Evolution 8:665-680

Patton JS, Abraham S, Benson AA (1977) Lipogenesis in the intact coral Pocillopora capitata and its isolated zooxanthellae: evidence for a light-driven carbon cycle between symbiont and host. Marine Biology 44:235-247

Peier AM, Moqrich A, Hergarden AC, Reeve AJ, Andersson DA, Story GM, Earley TJ, Dragoni I, McIntyre P, Bevan S (2002) A TRP channel that senses cold stimuli and menthol. Cell 108:705-715

Peleg-Grossman S, Volpin H, Levine A (2007) Root hair curling and Rhizobium infection in Medicago truncatula are mediated by phosphatidylinositide-regulated endocytosis and reactive oxygen species. Journal of Experimental Botany 58:1637-1649 
Peng SE, Wang YB, Wang LH, Chen WNU, Lu CY, Fang LS, Chen CS (2010) Proteomic analysis of symbiosome membranes in Cnidaria-dinoflagellate endosymbiosis. Proteomics 10:1002-1016

Pernice M, Meibom A, Van Den Heuvel A, Kopp C, Domart-Coulon I, Hoegh-Guldberg O, Dove S (2012) A single-cell view of ammonium assimilation in coral-dinoflagellate symbiosis. The ISME Journal 6:13141324

Pernice M, Levy O (2014) Novel tools integrating metabolic and gene function to study the impact of the environment on coral symbiosis. Frontiers in microbiology 5:448

Pernice M, Dunn SR, Tonk L, Dove S, Domart - Coulon I, Hoppe P, Schintlmeister A, Wagner M, Meibom A (2015) A nanosims study of dinoflagellate functional diversity in reef - building corals. Environmental Microbiology 17:3570-3580

Pettay DT, Wham DC, PINZoN JH, Lajeunesse TC (2011) Genotypic diversity and spatial-temporal distribution of Symbiodinium clones in an abundant reef coral. Molecular Ecology 20:5197-5212

Pettay DT, Wham DC, Smith RT, Iglesias-Prieto R, LaJeunesse TC (2015) Microbial invasion of the Caribbean by an Indo-Pacific coral zooxanthella. Proceedings of the National Academy of Sciences 112:7513-7518

Phelps TJ, Palumbo AV, Beliaev AS (2002) Metabolomics and microarrays for improved understanding of phenotypic characteristics controlled by both genomics and environmental constraints. Current Opinion in Biotechnology 13:20-24

Pochon X, Gates RD (2010) A new Symbiodinium clade (Dinophyceae) from soritid foraminifera in Hawai'i. Molecular Phylogenetics and Evolution 56:492-497

Pochon X, Stat M, Takabayashi M, Chasqui L, Chauka LJ, Logan DDK, Gates RD (2010) Comparison of endosymbiotic and free-living Symbiodinium (Dinophyceae) diversity in a Hawaiian reef environment. Journal of Phycology 46:53-65

Poole AZ, Kitchen SA, Weis VM (2016) The role of complement in cnidarian-dinoflagellate symbiosis and immune challenge in the sea anemone Aiptasia pallida. Frontiers in microbiology 7:519

Porto I, Granados C, Restrepo JC, Sanchez JA (2008) Macroalgal-associated dinoflagellates belonging to the genus Symbiodinium in Caribbean reefs. PloS ONE 3:e2160

Pratchett MS, McCowan D, Maynard JA, Heron SF (2013) Changes in bleaching susceptibility among corals subject to ocean warming and recurrent bleaching in Moorea, French Polynesia. PloS ONE 8:e70443

Putnam HM, Stat M, Pochon X, Gates RD (2012) Endosymbiotic flexibility associates with environmental sensitivity in scleractinian corals. Proceedings of the Royal Society B: Biological Sciences :rspb20121454

Rader BA, Nyholm SV (2012) Host/microbe interactions revealed through "omics" in the symbiosis between the Hawaiian bobtail squid Euprymna scolopes and the bioluminescent bacterium Vibrio fischeri. The Biological Bulletin 223:103-111

Rands ML, Loughman BC, Douglas AE (1993) The symbiotic interface in an alga-invertebrate symbiosis. Proceedings of the Royal Society of London B: Biological Sciences 253:161-165

Rasmussen S, Parsons AJ, Newman JA (2009) Metabolomics analysis of the Lolium perenne-Neotyphodium lolii symbiosis: more than just alkaloids? Phytochemistry Reviews 8:535-550

Rees TAV (1989) The green hydra symbiosis and ammonium II. Ammonium assimilation and release by freshly isolated symbionts and cultured algae. Proceedings of the Royal society of London Series B Biological sciences 235:365-382

Reid G, Babes A, Pluteanu F (2002) A cold - and menthol - activated current in rat dorsal root ganglion neurones: properties and role in cold transduction. The Journal of Physiology 545:595-614

Reynolds JM, Bruns BU, Fitt WK, Schmidt GW (2008) Enhanced photoprotection pathways in symbiotic dinoflagellates of shallow-water corals and other cnidarians. Proceedings of the National Academy of Sciences 105:13674-13678

Richier S, Furla P, Plantivaux A, Merle P-L, Allemand D (2005) Symbiosis-induced adaptation to oxidative stress. Journal of Experimental Biology 208:277-285

Richier S, Sabourault C, Courtiade J, Zucchini N, Allemand D, Furla P (2006) Oxidative stress and apoptotic events during thermal stress in the symbiotic sea anemone, Anemonia viridis. Febs Journal 273:41864198

Ritson-Williams R, Gates RD (2016) Kaneohe Bay Seawater Temperature Data 2014 and 2015 [Data set]. Zenodo

Roberts JM, Davies PS, Fixter LM, Preston T (1999) Primary site and initial products of ammonium assimilation in the symbiotic sea anemone Anemonia viridis. Marine Biology 135:223-236

Roberts JM, Fixter LM, Davies PS (2001) Ammonium metabolism in the symbiotic sea anemone Anemonia viridis. Hydrobiologia 461:25-35

Roberty S, Furla P, Plumier JC (2016) Differential antioxidant response between two Symbiodinium species from contrasting environments. Plant, Cell \& Environment 39:2713-2724

Rodrigues LJ, Grottoli AG (2007) Energy reserves and metabolism as indicators of coral recovery from bleaching. Limnology and Oceanography 52:1874-1882

Rodrigues LJ, Grottoli AG, Pease TK (2008) Lipid class composition of bleached and recovering Porites compressa Dana, 1846 and Montipora capitata Dana, 1846 corals from Hawaii. Journal of Experimental Marine Biology and Ecology 358:136-143 
Rodriguez-Lanetty M, Chang S-J, Song J-I (2003) Specificity of two temperate dinoflagellate-anthozoan associations from the north-western Pacific Ocean. Marine Biology 143:1193-1199

Rodriguez-Lanetty M, Krupp DA, Weis VM (2004) Distinct ITS types of Symbiodinium in Clade C correlate with cnidarian/dinoflagellate specificity during onset of symbiosis. Marine Ecology Progress Series 275:97102

Rodriguez-Lanetty M, Phillips WS, Weis VM (2006a) Transcriptome analysis of a cnidarian-dinoflagellate mutualism reveals complex modulation of host gene expression. BMC Genomics 7:23

Rodriguez-Lanetty M, Wood-Charlson EM, Hollingsworth LL, Krupp DA, Weis VM (2006b) Temporal and spatial infection dynamics indicate recognition events in the early hours of a dinoflagellate/coral symbiosis. Marine Biology 149:713-719

Roessner U, Wagner C, Kopka J, Trethewey RN, Willmitzer L (2000) Simultaneous analysis of metabolites in potato tuber by gas chromatography-mass spectrometry. The Plant Journal 23:131-142

Rohn H, Junker A, Hartmann A, Grafahrend-Belau E, Treutler H, Klapperstück M, Czauderna T, Klukas C, Schreiber F (2012) VANTED v2: a framework for systems biology applications. BMC Systems Biology 6:1

Rosic NN, Dove S (2011) Mycosporine-like amino acids from coral dinoflagellates. Applied and Environmental Microbiology 77:8478-8486

Rosic NN, Ling EYS, Chan C-KK, Lee HC, Kaniewska P, Edwards D, Dove S, Hoegh-Guldberg O (2015) Unfolding the secrets of coral-algal symbiosis. The ISME Journal 9:844-856

Roth KE, Jeon K, Stacey G (1988) Homology in endosymbiotic systems: the term "Symbiosome.”. In: Palacios R, Verma D (eds) Molecular Genetics of Plant-Microbe Interactions. APS Press, St. Paul, MN., pp220-225

Rowan R, Powers DA (1992) Ribosomal RNA sequences and the diversity of symbiotic dinoflagellates (zooxanthellae). Proceedings of the National Academy of Sciences 89:3639-3643

Rowan R, Knowlton N (1995) Intraspecific diversity and ecological zonation in coral-algal symbiosis. Proceedings of the National Academy of Sciences 92:2850-2853

Rowan R, Knowlton N, Baker A, Jara J (1997) Landscape ecology of algal symbionts creates variation in episodes of coral bleaching. Nature 388:265-269

Rowan R (2004) Coral bleaching: thermal adaptation in reef coral symbionts. Nature 430:742-742

Rumble SM, Lacroute P, Dalca AV, Fiume M, Sidow A, Brudno M (2009) SHRiMP: accurate mapping of short color-space reads. PLoS Computational Biology 5:e1000386

Sachs JL, Wilcox TP (2006) A shift to parasitism in the jellyfish symbiont Symbiodinium microadriaticum. Proceedings of the Royal Society of London B: Biological Sciences 273:425-429

Sachs JL, Skophammer RG, Regus JU (2011) Evolutionary transitions in bacterial symbiosis. Proceedings of the National Academy of Sciences 108:10800-10807

Sampayo EM, Franceschinis L, Hoegh-Guldberg O, Dove S (2007) Niche partitioning of closely related symbiotic dinoflagellates. Molecular Ecology 16: 3721-3733

Sampayo EM, Ridgway T, Bongaerts P, Hoegh-Guldberg O (2008a) Bleaching susceptibility and mortality of corals are determined by fine-scale differences in symbiont type. Proceedings of the National Academy of Sciences 105: 10444-10449

Sampayo EM, Ridgway T, Bongaerts P, Hoegh-Guldberg O (2008b) Bleaching susceptibility and mortality of corals are determined by fine-scale differences in symbiont type. Proceedings of the National Academy of Sciences of the United States of America 105:10444-10449

Sampayo EM, Dove S, Lajeunesse TC (2009) Cohesive molecular genetic data delineate species diversity in the dinoflagellate genus Symbiodinium. Molecular Ecology 18:500-519

Sampayo EM, Ridgway T, Franceschinis L, Roff G, Hoegh-Guldberg O, Dove S (2016) Coral symbioses under prolonged environmental change: living near tolerance range limits. Scientific Reports 6:36271

Santos SR, Shearer TL, Hannes AR, Coffroth MA (2004) Fine - scale diversity and specificity in the most prevalent lineage of symbiotic dinoflagellates (Symbiodinium, Dinophyceae) of the Caribbean. Molecular Ecology 13:459-469

Santos SR, Toyoshima J, KINZIE III RA (2009) Spatial and temporal dynamics of symbiotic dinoflagellates (Symbiodinium: Dinophyta) in the perforate coral Montipora capitata. Galaxea, Journal of Coral Reef Studies 11:139-147

Saunders SM, Radford B, Bourke SA, Thiele Z, Bech T, Mardon J (2006) A rapid method for determining lipid fraction ratios of hard corals under varying sediment and light regimes. Environmental Chemistry 2:331336

Savchenko T, Walley JW, Chehab EW, Xiao Y, Kaspi R, Pye MF, Mohamed ME, Lazarus CM, Bostock RM, Dehesh K (2010) Arachidonic acid: an evolutionarily conserved signaling molecule modulates plant stress signaling networks. The Plant Cell 22:3193-3205

Sawyer SJ, Muscatine L (2001) Cellular mechanisms underlying temperature-induced bleaching in the tropical sea anemone Aiptasia pulchella. Journal of Experimental Biology 204:3443-3456 
Schauer N, Steinhauser D, Strelkov S, Schomburg D, Allison G, Moritz T, Lundgren K, Roessner-Tunali U, Forbes MG, Willmitzer L, Fernie AR, Kopka J (2005) GC-MS libraries for the rapid identification of metabolites in complex biological samples. FEBS letters 579:1332-1337

Schliemann W, Ammer C, Strack D (2008) Metabolite profiling of mycorrhizal roots of Medicago truncatula. Phytochemistry 69:112-146

Schlöder C, D'Croz L (2004) Responses of massive and branching coral species to the combined effects of water temperature and nitrate enrichment. Journal of Experimental Marine Biology and Ecology 313:255-268

Schmeitzel JL, Klein J, Smith M, Schwarz J, Garrett TA (2012) Comparative lipidomic analysis of the symbiosis between Aiptasia pallida and Symbiodinium. The FASEB Journal 26:789.781

Schnitzler CE, Weis VM (2010) Coral larvae exhibit few measurable transcriptional changes during the onset of coral-dinoflagellate endosymbiosis. Marine Genomics 3:107-116

Schnitzler CE, Hollingsworth LL, Krupp DA, Weis VM (2012) Elevated temperature impairs onset of symbiosis and reduces survivorship in larvae of the Hawaiian coral, Fungia scutaria. Marine Biology 159:633-642

Schoenberg DA, Trench RK (1980a) Genetic Variation in Symbiodinium (= Gymnodinium) microadriaticum Freudenthal, and specificity in its symbiosis with marine invertebrates. III. Specificity and infectivity of Symbiodinium microadriaticum. Royal Society of London Proceedings Series B 207:445-460

Schoenberg DA, Trench RK (1980b) Genetic variation in Symbiodinium (= Gymnodinium) microadriaticum Freudenthal, and specificity in its symbiosis with marine invertebrates. I. Isoenzyme and soluble protein patterns of axenic cultures of Symbiodinium microadriaticum. Proceedings of the Royal Society of London Series B Biological Sciences 207:405-427

Schwarz JA, Krupp DA, Weis VM (1999) Late larval development and onset of symbiosis in the scleractinian coral Fungia scutaria. The Biological Bulletin 196:70-79

Scott C, Ioannou YA (2004) The NPC1 protein: structure implies function. Biochimica et Biophysica Acta (BBA)Molecular and Cell Biology of Lipids 1685:8-13

Sebens KP (2002) Energetic constraints, size gradients, and size limits in benthic marine invertebrates. Integrative and Comparative Biology 42:853-861

Shade A, Jones SE, Caporaso JG, Handelsman J, Knight R, Fierer N, Gilbert JA (2014) Conditionally rare taxa disproportionately contribute to temporal changes in microbial diversity. mBio 5:e01371-01314

Shastri AA, Morgan JA (2007) A transient isotopic labeling methodology for ${ }^{13} \mathrm{C}$ metabolic flux analysis of photoautotrophic microorganisms. Phytochemistry 68:2302-2312

Shick JM (2004) The continuity and intensity of ultraviolet irradiation affect the kinetics of biosynthesis Accumulation and conversion of micosporine-like amino acids (MAAs) in the Sylophora Pistillata. Limnology and Oceanography 49:442-458

Shick JM, Dunlap WC (2002) Mycosporine-like amino acids and related gadusols: biosynthesis, accumulation, and UV-protective functions in aquatic organisms. Annual Review of Physiology 64:223-262

Shinzato C, Shoguchi E, Kawashima T, Hamada M, Hisata K, Tanaka M, Fujie M, Fujiwara M, Koyanagi R, Ikuta $\mathrm{T}$ (2011) Using the Acropora digitifera genome to understand coral responses to environmental change. Nature 476:320-323

Shoguchi E, Tanaka M, Takeuchi T, Shinzato C, Satoh N (2013a) Probing a coral genome for components of the photoprotective scytonemin biosynthetic pathway and the 2-aminoethylphosphonate pathway. Marine Drugs 11:559-570

Shoguchi E, Shinzato C, Kawashima T, Gyoja F, Mungpakdee S, Koyanagi R, Takeuchi T, Hisata K, Tanaka M, Fujiwara M, Hamada M, Seidi A, Fujie M, Usami T, Goto H, Yamasaki S, Arakaki N, Suzuki Y, Sugano S, Toyoda A, Kuroki Y, Fujiyama A, Medina M, Coffroth MA, Bhattacharya D, Satoh N (2013b) Draft assembly of the Symbiodinium minutum nuclear genome reveals dinoflagellate gene structure. Current Biology 23:1399-1408

Silverstein RN, Correa AM, Baker AC (2012) Specificity is rarely absolute in coral-algal symbiosis: implications for coral response to climate change. Proceedings of The Royal Society of London Biological Sciences 279:2609-2618

Silverstein RN, Cunning R, Baker AC (2015) Change in algal symbiont communities after bleaching, not prior heat exposure, increases heat tolerance of reef corals. Global Change Biology 21:236-249

Smart KF, Aggio RB, Van Houtte JR, Villas-Boas SG (2010) Analytical platform for metabolome analysis of microbial cells using methyl chloroformate derivatization followed by gas chromatography-mass spectrometry. Nature Protocols 5:1709-1729

Smith DC, Douglas AE (1987) The biology of symbiosis. Edward Arnold (Publishers) Ltd.

Smith DC (1993) The symbiotic condition. Symbiosis 14:3-15

Smith EG, Ketchum RN, Burt JA (2017) Host specificity of Symbiodinium variants revealed by an ITS2 metahaplotype approach. The ISME Journal. 11:1500-1503

Smith G, Muscatine L (1986) Carbon budgets and regulation of the population-density of symbiotic algae. Endocytobiosis and Cell Research 3:213-238 
Smith G, Muscatine L (1999) Cell cycle of symbiotic dinoflagellates: variation in G1 phase-duration with anemone nutritional status and macronutrient supply in the Aiptasia pulchella-Symbiodinium pulchrorum symbiosis. Marine Biology 134:405-418

Sogin EM, Putnam HM, Anderson PE, Gates RD (2016) Metabolomic signatures of increases in temperature and ocean acidification from the reef-building coral, Pocillopora damicornis. Metabolomics 12:1-12

Stanley Jr GD (2006) Photosymbiosis and the evolution of modern coral reefs. Evolution; International Journal of Organic Evolution 1:3

Starzak DE, Quinnell RG, Nitschke MR, Davy SK (2014) The influence of symbiont type on photosynthetic carbon flux in a model cnidarian-dinoflagellate symbiosis. Marine Biology 161:711-724

Stat M, Morris E, Gates RD (2008) Functional diversity in coral-dinoflagellate symbiosis. Proceedings of the National Academy of Sciences of the United States of America 105:9256-9261

Stat M, Loh WKW, LaJeunesse TC, Hoegh-Guldberg O, Carter DA (2009) Stability of coral-endosymbiont associations during and after a thermal stress event in the southern Great Barrier Reef. Coral Reefs 28:709-713

Stat M, Gates RD (2011) Clade D Symbiodinium in scleractinian corals: A "nugget" of hope, a selfish opportunist, an ominous sign, or all of the above? Journal of Marine Biology 2011:1-9

Stat M, Bird CE, Pochon X, Chasqui L, Chauka LJ, Concepcion GT, Logan D, Takabayashi M, Toonen RJ, Gates RD (2011) Variation in Symbiodinium ITS2 sequence assemblages among coral colonies. PloS ONE 6:e15854

Stat M, Pochon X, Franklin EC, Bruno JF, Casey KS, Selig ER, Gates RD (2013) The distribution of the thermally tolerant symbiont lineage (Symbiodinium clade D) in corals from Hawaii: correlations with host and the history of ocean thermal stress. Ecology and Evolution 3:1317-1329

Stat M, Yost DM, Gates RD (2015) Geographic structure and host specificity shape the community composition of symbiotic dinoflagellates in corals from the Northwestern Hawaiian Islands. Coral Reefs 34:1075-1086

Stat M, Baker AC, Bourne DG, Correa AM, Forsman Z, Huggett MJ, Pochon X, Skillings D, Toonen RJ, van Oppen MJH (2012) 1 Molecular delineation of species in the coral holobiont. Advances in Marine Biology 63:1

Steen RG, Muscatine L (1987) Low temperature evokes rapid exocytosis of symbiotic algae by a sea anemone. The Biological Bulletin 172:246-263

Stern RF, Horak A, Andrew RL, Coffroth M-A, Andersen RA, Küpper FC, Jameson I, Hoppenrath M, Véron B, Kasai F (2010) Environmental barcoding reveals massive dinoflagellate diversity in marine environments. PloS ONE 5:e13991

Strychar KB, Sammarco PW (2009) Exaptation in corals to high seawater temperatures: Low concentrations of apoptotic and necrotic cells in host coral tissue under bleaching conditions. Journal of Experimental Marine Biology and Ecology 369:31-42

Strychar KB, Coates M, Sammarco PW, Piva TJ (2004) Bleaching as a pathogenic response in scleractinian corals, evidenced by high concentrations of apoptotic and necrotic zooxanthellae. Journal of Experimental Marine Biology and Ecology 304:99-121

Sunagawa S, Wilson EC, Thaler M, Smith ML, Caruso C, Pringle JR, Weis VM, Medina M, Schwarz JA (2009a) Generation and analysis of transcriptomic resources for a model system on the rise: the sea anemone Aiptasia pallida and its dinoflagellate endosymbiont. BMC Genomics 10:258

Sutton DC, Hoegh-Guldberg O (1990) Host-zooxanthella interactions in four temperate marine invertebrate symbioses: assessment of effect of host extracts on symbionts. The Biological Bulletin 178:175-186

Swanson R, Hoegh-Guldberg O (1998) Amino acid synthesis in the symbiotic sea anemone Aiptasia pulchella. Marine Biology 131:83-93

Takabayashi M, Adams LM, Pochon X, Gates RD (2012) Genetic diversity of free-living Symbiodinium in surface water and sediment of Hawai 'i and Florida. Coral Reefs 31:157-167

Tanaka Y, Miyajima T, Koike I, Hayashibara T, Ogawa H (2006) Translocation and conservation of organic nitrogen within the coral-zooxanthella symbiotic system of Acropora pulchra, as demonstrated by dual isotope-labeling techniques. Journal of Experimental Marine Biology and Ecology 336:110-119

Taylor DL (1974) Symbiotic marine algae: taxonomy and biological fitness. Symbiosis in the Sea pp. 245-262

Tchernov D, Gorbunov MY, de Vargas C, Narayan Yadav S, Milligan AJ, Haggblom M, Falkowski PG (2004) Membrane lipids of symbiotic algae are diagnostic of sensitivity to thermal bleaching in corals. Proceedings of the National Academy of Sciences of the United States of America 101:13531-13535

Tchernov D, Kvitt H, Haramaty L, Bibby TS, Gorbunov MY, Rosenfeld H, Falkowski PG (2011) Apoptosis and the selective survival of host animals following thermal bleaching in zooxanthellate corals. Proceedings of the National Academy of Sciences 108:9905-9909

Teece MA, Estes B, Gelsleichter E, Lirman D (2011) Heterotrophic and autotrophic assimilation of fatty acids by two scleractinian corals, Montastraea faveolata and Porites astreoides. Limnology and Oceanography 56:1285

Thornhill D, Howells E, Wham D, Steury T, Santos S (2017) Population genetics of reef coral endosymbionts (Symbiodinium, Dinophyceae). Molecular ecology 26:2640-2659 
Thornhill DJ, LaJeunesse TC, Kemp DW, Fitt WK, Schmidt GW (2006) Multi-year, seasonal genotypic surveys of coral-algal symbioses reveal prevalent stability or post-bleaching reversion. Marine Biology 148:711-722

Thornhill DJ, Lajeunesse TC, Santos SR (2007) Measuring rDNA diversity in eukaryotic microbial systems: how intragenomic variation, pseudogenes, and PCR artifacts confound biodiversity estimates. Molecular Ecology 16:5326-5340

Thornhill DJ, Xiang Y, Fitt WK, Santos SR (2009) Reef endemism, host specificity and temporal stability in populations of symbiotic dinoflagellates from two ecologically dominant Caribbean corals. PloS ONE 4:e6262

Thornhill DJ, Xiang Y, Pettay DT, Zhong M, Santos SR (2013) Population genetic data of a model symbiotic cnidarian system reveal remarkable symbiotic specificity and vectored introductions across ocean basins. Molecular Ecology 22:4499-4515

Thornhill DJ, Lewis AM, Wham DC, LaJeunesse TC (2014) Host-specialist lineages dominate the adaptive radiation of reef coral endosymbionts. Evolution; international journal of organic evolution 68:352-367

Thrall PH, Hochberg ME, Burdon JJ, Bever JD (2006) Coevolution of symbiotic mutualists and parasites in a community context. Trends in Ecology and Evolution 22:120-126

Tremblay P, Grover R, Maguer JF, Legendre L, Ferrier-Pages C (2012) Autotrophic carbon budget in coral tissue: a new ${ }^{13} \mathrm{C}$-based model of photosynthate translocation. The Journal of Experimental Biology 215:13841393

Tremblay P, Grover R, Maguer JF, Hoogenboom M, Ferrier-Pagès C (2014) Carbon translocation from symbiont to host depends on irradiance and food availability in the tropical coral Stylophora pistillata. Coral Reefs 33:1-13

Tremblay P, Maguer JF, Grover R, Ferrier-Pagès C (2015) Trophic dynamics of scleractinian corals: stable isotope evidence. Journal of Experimental Biology 218:1223-1234

Tremblay P, Gori A, Maguer JF, Hoogenboom M, Ferrier-Pagès C (2016) Heterotrophy promotes the reestablishment of photosynthate translocation in a symbiotic coral after heat stress. Scientific Reports 6:38112

Trench RK (1971a) The physiology and biochemistry of zooxanthellae symbiotic with marine coelenterates. III. The effect of homogenates of host tissues on the excretion of photosynthetic products in vitro by zooxanthellae from two marine coelenterates. Proceedings of the Royal Society of London Series B Biological Sciences 177:251-264

Trench RK (1971b) The physiology and biochemistry of zooxanthellae symbiotic with marine coelenterates. I. The assimilation of photosynthetic products of zooxanthellae by two marine coelenterates. Proceedings of the Royal Society of London Series B Biological Sciences 177:225-235

Trench RK (1971c) The Physiology and Biochemistry of Zooxanthellae Symbiotic with Marine Coelenterates. II. Liberation of Fixed ${ }^{14} \mathrm{C}$ by Zooxanthellae in vitro. Proceedings of the Royal Society of London Series B Biological Sciences 177:237-250

Trench RK (1979) The cell biology of plant-animal symbiosis. Annual Review of Plant Physiology 30:485-531

Trench RK (1987) Dinoflagellates in non-parasitic symbioses. In: F.J.R. Taylor (ed.) The Biology of Dinoflagellates. Blackwell, Oxford, pp. 530-570

Trench RK (1997) Diversity of symbiotic dinoflagellates and the evolution of microalgal-invertebrate symbioses. Proc 8th Int Coral Reef Symp 2:1275-1286

Tsim S-T, Wong J, Wong YH (1997) Calcium ion dependency and the role of inositol phosphates in melatonininduced encystment of dinoflagellates. Journal of Cell Science 110:1387-1393

Ulstrup KE, Berkelmans R, Ralph PJ, van Oppen MJH (2006) Variation in bleaching sensitivity of two coral species across a latitudinal gradient on the Great Barrier Reef: the role of zooxanthellae. Marine Ecology Progress Series 314:135-148

UniProt Consortium (2014) UniProt: a hub for protein information. Nucleic Acids Research 43:204-212

Urich K (2013) Comparative animal biochemistry. Springer Science \& Business Media

Vacelet J (1981) Algal-sponge symbioses in the coral reefs of New Caledonia: a morphological study. Proc 4th Int Coral Reef Symp Manila 2:713-719

Vance JE (2010) Transfer of cholesterol by the NPC team. Cell Metabolism 12:105-106

van Oppen MJH, Baker AC, Coffroth MA, Willis BL (2009) Bleaching resistance and the role of algal endosymbionts. In: Lough, J.M., van Oppen, M.J.H. (Eds.), Coral Bleaching: Patterns, Processes, Causes and Consequences. Springer Ecological Studies, Berlin, pp. 84-102.

van Woesik R, Sakai K, Ganase A, Loya Y (2011) Revisiting the winners and the losers a decade after coral bleaching. Marine Ecology Progress Series 434:67-76

Venables WN, Smith DM (2005) the R development core team. An Introduction to R Notes on R: A Programming Environment for Data Analysis and Graphics

Venera-Ponton DE, Diaz-Pulido G, Rodriguez-Lanetty M, Hoegh-Guldberg O (2010) Presence of Symbiodinium spp. in macroalgal microhabitats from the southern Great Barrier Reef. Coral Reefs 29:1049-1060 
Venn AA, Loram JE, Douglas AE (2008) Photosynthetic symbioses in animals. Journal of Experimental Botany 59:1069-1080

Viant MR (2007) Metabolomics of aquatic organisms: the new'omics' on the block. Marine Ecology Progress Series 332:301-306

Vinaixa M, Samino S, Saez I, Duran J, Guinovart JJ, Yanes O (2012) A guideline to univariate statistical analysis for LC/MS-based untargeted metabolomics-derived data. Metabolites 2:775-795

Vitelli R, Santillo M, Lattero D, Chiariello M, Bifulco M, Bruni CB, Bucci C (1997) Role of the small GTPase Rab7 in the late endocytic pathway. Journal of Biological Chemistry 272:4391-4397

Volkman J, Jeffrey S, Nichols P, Rogers G, Garland C (1989) Fatty acid and lipid composition of 10 species of microalgae used in mariculture. Journal of Experimental Marine Biology and Ecology 128:219-240

Von Holt C, Von Holt M (1968a) The secretion of organic compounds by zooxanthellae isolated from various types of Zoanthus. Comparative biochemistry and physiology 24:83-92

Von Holt C, Von Holt M (1968b) Transfer of photosynthetic products from zooxanthellae to coelenterate hosts. Comparative biochemistry and physiology 24:73-81

Voolstra CR, Schwarz JA, Schnetzer J, Sunagawa S, Desalvo MK, Szmant AM, Coffroth MA, Medina M (2009) The host transcriptome remains unaltered during the establishment of coral-algal symbioses. Molecular ecology 18:1823-1833

Voolstra CR (2013) A journey into the wild of the cnidarian model system Aiptasia and its symbionts. Molecular ecology 22:4366-4368

Wakefield TS, Kempf SC (2001) Development of host-and symbiont-specific monoclonal antibodies and confirmation of the origin of the symbiosome membrane in a cnidarian-dinoflagellate symbiosis. The Biological Bulletin 200:127-143

Wakefield TS, Farmer MA, Kempf SC (2000) Revised description of the fine structure of in situ" zooxanthellae" genus Symbiodinium. The Biological Bulletin 199:76-84

Wang JT, Douglas AE (1997) Nutrients, signals, and photosynthate release by symbiotic algae (the impact of taurine on the dinoflagellate alga Symbiodinium from the sea anemone Aiptasia pulchella). Plant Physiology 114:631-636

Wang JT, Douglas AE (1998) Nitrogen recycling or nitrogen conservation in an alga-invertebrate symbiosis? The Journal of Experimental Biology 201:2445-2453

Wang JT, Douglas AE (1999) Essential amino acid synthesis and nitrogen recycling in an alga-invertebrate symbiosis. Marine Biology 135:219-222

Wang JT, Chen YY, Tew KS, Meng PJ, Chen CA (2012) Physiological and biochemical performances of mentholinduced aposymbiotic corals. PloS ONE 7:e46406

Wang LH, Lee HH, Fang LS, Mayfield AB, Chen CS (2013) Fatty acid and phospholipid syntheses are prerequisites for the cell cycle of Symbiodinium and their endosymbiosis within sea anemones. PloS ONE 8:e72486

Wang Y, Dunn AK, Wilneff J, McFall - Ngai MJ, Spiro S, Ruby EG (2010) Vibrio fischeri flavohaemoglobin protects against nitric oxide during initiation of the squid-Vibrio symbiosis. Molecular Microbiology 78:903-915

Warner ME, Fitt WK, Schmidt GW (1999) Damage to photosystem II in symbiotic dinoflagellates: a determinant of coral bleaching. Proceedings of the National Academy of Sciences 96:8007-8012

Weindl D, Wegner A, Hiller K (2015) Chapter Eight-Non-targeted Tracer Fate Detection. Methods in Enzymology 561:277-302

Weindl D, Wegner A, Hiller K (2016a) MIA: Non-targeted mass isotopolome analysis. Bioinformatics 32:28752876

Weindl D, Cordes T, Battello N, Sapcariu SC, Dong X, Wegner A, Hiller K (2016b) Bridging the gap between non-targeted stable isotope labeling and metabolic flux analysis. Cancer \& metabolism 4:1

Weis V, Smith G, Muscatine L (1989) A “ $\mathrm{CO}_{2}$ supply” mechanism in zooxanthellate cnidarians: role of carbonic anhydrase. Marine Biology 100:195-202

Weis VM (1991) The induction of carbonic anhydrase in the symbiotic sea anemone Aiptasia pulchella. The Biological Bulletin 180:496-504

Weis VM, Reynolds WS (1999) Carbonic anhydrase expression and synthesis in the sea anemone Anthopleura elegantissima are enhanced by the presence of dinoflagellate symbionts. Physiological and Biochemical Zoology 72:307-316

Weis V, Reynolds W, deBoer M, Krupp D (2001) Host-symbiont specificity during onset of symbiosis between the dinoflagellates Symbiodinium spp. and planula larvae of the scleractinian coral Fungia scutaria. Coral Reefs 20:301-308

Weis VM (2008) Cellular mechanisms of Cnidarian bleaching: stress causes the collapse of symbiosis. Journal of Experimental Biology 211:3059-3066

Weis VM, Davy SK, Hoegh-Guldberg O, Rodriguez-Lanetty M, Pringle JR (2008) Cell biology in model systems as the key to understanding corals. Trends in Ecology and Evolution 23:369-376

Weis VM, Allemand D (2009) Physiology. What determines coral health? Science 324:1153-1155 
Wham DC, LaJeunesse TC (2016) Symbiodinium population genetics: testing for species boundaries and analysing samples with mixed genotypes. Molecular Ecology 12:2699-2712

Whatley JM, John P, Whatley F (1979) From extracellular to intracellular: the establishment of mitochondria and chloroplasts. Proceedings of the Royal Society of London Series B, Biological Sciences 204:165-187

Whatley JM, Whatley F (1981) Chloroplast evolution. New Phytologist 87:233-247

Whitehead LF, Douglas AE (2003) Metabolite comparisons and the identity of nutrients translocated from symbiotic algae to an animal host. Journal of Experimental Biology 206:3149-3157

Whitney SM, Yellowlees D (1995) Preliminary investigations into the structure and activity of ribulose bisphosphate carboxylase from two photosynthetic dinoflagellates. Journal of Phycology 31:138-146

Wicks LC, Gardner JPA, Davy SK (2011) Host tolerance, not symbiont tolerance, determines the distribution of coral species in relation to their environment at a Central Pacific atoll. Coral Reefs 31:389-398

Wietheger A (2012) Coral bleaching resistance vs susceptibility: The role of antioxidant activity in symbiotic Dinoflagellates. PhD Thesis, Victoria University of Wellington

Wietheger A, Fisher PL, Gould KS, Davy SK (2015) Sensitivity to oxidative stress is not a definite predictor of thermal sensitivity in symbiotic dinoflagellates. Marine Biology 162:2067-2077

Wilkinson SP, Fisher PL, van Oppen MJH, Davy SK (2015) Intra-genomic variation in symbiotic dinoflagellates: recent divergence or recombination between lineages? BMC Evolutionary Biology 15:1

Withers KJ, Grant AJ, Hinde R (1998) Effects of free amino acids on the isolated symbiotic algae of the coral Plesiastrea versipora (Lamarck): absence of a host release factor response. Comparative Biochemistry and Physiology Part A: Molecular \& Integrative Physiology 120:599-607

Wolinska J, King KC (2009) Environment can alter selection in host- parasite interactions. Trends in Parasitology 25:236-244

Wood - Charlson EM, Hollingsworth LL, Krupp DA, Weis VM (2006) Lectin/glycan interactions play a role in recognition in a coral/dinoflagellate symbiosis. Cellular microbiology 8:1985-1993

Wood-Charlson EM, Weis VM (2009) The diversity of C-type lectins in the genome of a basal metazoan, Nematostella vectensis. Developmental \& Comparative Immunology 33:881-889

Wooldridge SA (2009) Water quality and coral bleaching thresholds: Formalising the linkage for the inshore reefs of the Great Barrier Reef, Australia. Marine Pollution Bulletin 58:745-751

Wright EM, Turk E (2004) The sodium/glucose cotransport family SLC5. Pflügers Archiv 447:510-518

Wright RM, Aglyamova GV, Meyer E, Matz MV (2015) Gene expression associated with white syndromes in a reef building coral, Acropora hyacinthus. BMC Genomics 16:371

Xia J, Wishart DS (2011) Metabolomic data processing, analysis, and interpretation using MetaboAnalyst. Current protocols in bioinformatics 48:14-10

Xia J, Sinelnikov IV, Han B, Wishart DS (2015) MetaboAnalyst 3.0-making metabolomics more meaningful. Nucleic Acids Research 43:251-257

Xiang T, Hambleton EA, DeNofrio JC, Pringle JR, Grossman AR, Lin S (2013) Isolation of clonal axenic strains of the symbiotic dinoflagellate Symbiodinium and their growth and host specificity. Journal of Phycology 49:447-458

Yakovleva IM, Baird AH, Yamamoto HH, Bhagooli R, Nonaka M, Hidaka M (2009) Algal symbionts increase oxidative damage and death in coral larvae at high temperatures. Marine Ecological Progress Series 378: $105-112$

Yang K, Li J, Gao H (2006) The impact of sample imbalance on identifying differentially expressed genes. $B M C$ Bioinformatics 7:1

Yamashiro H, Oku H, Higa H, Chinen I, Sakai K (1999) Composition of lipids, fatty acids and sterols in Okinawan corals. Comparative Biochemistry and Physiology Part B: Biochemistry and Molecular Biology 122:397407

Yellowlees D, Rees TAV, Fitt WK (1994) Effect of ammonium-supplemented seawater on glutamine synthetase and glutamate dehydrogenase activities in host tissue and zooxanthellae of Pocillopora damicornis and on ammonium uptake rates of the zooxanthellae. Pacific Science 48:291-295

Yellowlees D, Warner M (2003) Photosynthesis in symbiotic algae. In: Larkum, Anthony W.D., Douglas, Susan E., and Raven, John A., (eds.) Photosynthesis in Algae. Advances in Photosynthesis and Respiration, 14. Kluwer Academic Publishers, Dordrecht, The Netherlands, pp. 437-455

Yellowlees D, Rees TAV, Leggat W (2008) Metabolic interactions between algal symbionts and invertebrate hosts. Plant, Cell \& Environment 31:679-694

Yu L, Alva A, Su H, Dutt P, Freundt E, Welsh S, Baehrecke EH, Lenardo MJ (2004) Regulation of an ATG7beclin 1 program of autophagic cell death by caspase-8. Science 304:1500-1502

Yuyama I, Ito Y, Watanabe T, Hidaka M, Suzuki Y, Nishida M (2012) Differential gene expression in juvenile polyps of the coral Acropora tenuis exposed to thermal and chemical stresses. Journal of Experimental Marine Biology and Ecology 430:17-24

Zhang N, Venkateshwaran M, Boersma M, Harms A, Howes-Podoll M, den Os D, Ane JM, Sussman MR (2012) Metabolomic profiling reveals suppression of oxylipin biosynthesis during the early stages of legumerhizobia symbiosis. FEBS letters 586:3150-3158 
Zhukova NV, Aizdaicher NA (1995) Fatty acid composition of 15 species of marine microalgae. Phytochemistry 39:351-356

Zhukova NV, Titlyanov EA (2003) Fatty acid variations in symbiotic dinoflagellates from Okinawan corals. Phytochemistry 62:191-195

Zhukova NV, Titlyanov EA (2006) Effect of light intensity on the fatty acid composition of dinoflagellates symbiotic with hermatypic corals. Botanica Marina 49:339-346 


\section{SUPPLEMENTAL FIGURES AND TABLES}

Supplemental Table S3.1. Transcriptome assembly, mapping, and differential expression statistical values.

\section{De novo Transcriptome Assembly}

Number of reads after quality filtering

168,677

Total number of contigs

63,789

Total subcomponent isogroups

Total after filtering for low counts per transcript

Average contig length (bp)

Maximum contig length (bp)

Minimum contig length (bp)

N50 of all contigs (bp)

\section{Mapping}

Average number of reads per sample after filtering

\section{Differential Expression}

$\begin{array}{lr}\text { Number of transcripts passing thresholds and tested for differential expression } & 35,516 \\ \text { Number of transcripts differentially expressed with symbiont state }(p<0.05) & 514 \\ \text { Number of transcripts differentially expressed with symbiont state }(p<0.1) & 720 \\ \text { Number of transcripts differentially expressed after symbiont transcripts } & 686 \\ \text { removed } & 60 \% \\ \text { Percentage of differentially expressed transcripts with UniProt matches }\end{array}$


Supplementary Table S3.2. Mean quantitative data of the metabolites identified in the host tissues of Aiptasia when aposymbiotic, or colonised with homologous (type B1), or heterologous (type D1a) Symbiodinium. Data are mean relative abundances \pm S.E.M. $(n=5)$.

\begin{tabular}{|c|c|c|c|}
\hline Metabolite & Aposymbiotic & $\begin{array}{c}\text { Homologous type } \\
\text { B1 }\end{array}$ & $\begin{array}{c}\text { Heterologous } \\
\text { type D1a }\end{array}$ \\
\hline 1-Monooleoylglycerol & $2.888+0.132$ & $0.852+0.035$ & $1.589 \pm 0.363$ \\
\hline Adenosine & $2.826+1.433$ & $6.059 \pm 3.113$ & $13.713 \pm 7.951$ \\
\hline Adipic acid & $0.447 \pm 0.109$ & $0.095 \pm 0.038$ & $0.096 \pm 0.043$ \\
\hline Alanine & $25.403+2.076$ & $12.099+0.575$ & $17.356+1.036$ \\
\hline Alanine, beta & $6.282 \pm 0.994$ & $4.685 \pm 0.425$ & $6.628 \pm 0.317$ \\
\hline Arachidonic acid (C20:4) & $1.654 \pm 0.177$ & $1.775 \pm 0.123$ & $2.595 \pm 0.505$ \\
\hline Arachidyl alcohol & $0.039 \pm 0.012$ & $0.035 \pm 0.009$ & $0.049 \pm 0.019$ \\
\hline Aspartic acid & $4.749 \pm 0.874$ & $0.754 \pm 0.173$ & $1.565 \pm 0.473$ \\
\hline Butyric acid & $2.570 \pm 0.874$ & $0.114+0.007$ & $0.385 \pm 0.059$ \\
\hline Campesterol & $0.024+0.008$ & $0.014+0.004$ & $0.026 \pm 0.009$ \\
\hline Cholesterol & $1.736 \pm 0.581$ & $1.044 \pm 0.318$ & $1.393 \pm 0.515$ \\
\hline Citric acid & $1.395 \pm 0.168$ & $0.670 \pm 0.136$ & $0.886 \pm 0.218$ \\
\hline Cysteine & $1.621 \pm 0.550$ & $0.145 \pm 0.035$ & $0.295 \pm 0.098$ \\
\hline Cytosine & $0.263 \pm 0.029$ & $0.153 \pm 0.013$ & $0.351 \pm 0.172$ \\
\hline Docosahexaenoic acid (C22:6n-3) & $2.903 \pm 0.235$ & $2.199 \pm 0.169$ & $3.152+0.440$ \\
\hline Eicosanoic acid (C20:0) & $0.016 \pm 0.001$ & $0.026 \pm 0.016$ & $0.021 \pm 0.007$ \\
\hline Eicosapentaenoic acid (C20:5) & $12.843 \pm 1.023$ & $6.314 \pm 0.665$ & $11.484 \pm 1.360$ \\
\hline Eicosatrienoic acid (C20:3) & $0.269 \pm 0.075$ & $0.175 \pm 0.052$ & $0.350 \pm 0.084$ \\
\hline Erythrose & $1.182 \pm 0.162$ & $1.881 \pm 0.095$ & $1.623 \pm 0.137$ \\
\hline Ethanolamine & $0.440 \pm 0.061$ & $0.359 \pm 0.046$ & $0.567 \pm 0.191$ \\
\hline Fructose & $1.094 \pm 0.289$ & $0.356 \pm 0.105$ & $0.600 \pm 0.227$ \\
\hline Fructose-6-phosphate & $0.716 \pm 0.113$ & $0.134 \pm 0.037$ & $0.420 \pm 0.190$ \\
\hline Galactose & $0.248 \pm 0.063$ & $0.316 \pm 0.051$ & $0.350 \pm 0.091$ \\
\hline Glucopyranose & $0.172 \pm 0.0 .36$ & $0.125 \pm 0.095$ & $0.147 \pm 0.068$ \\
\hline Glucosamine & $0.908 \pm 0.164$ & $0.525 \pm 0.069$ & $1.312 \pm 0.360$ \\
\hline Glucose & $1.333 \pm 0.333$ & $1.572 \pm 0.264$ & $1.645 \pm 0.404$ \\
\hline Glutamate & $0.890 \pm 0.240$ & $0.947 \pm 0.134$ & $0.846 \pm 0.099$ \\
\hline Glycerate 3-phosphate & $1.935 \pm 0.356$ & $0.277 \pm 0.027$ & $0.607 \pm 0.250$ \\
\hline Glyceric acid & $0.269 \pm 0.039$ & $0.446 \pm 0.019$ & $0.661 \pm 0.075$ \\
\hline Glycerol & $0.133 \pm 0.042$ & $0.085 \pm 0.011$ & $0.120 \pm 0.016$ \\
\hline Glycerol 1-hexadecanoate & $0.170 \pm 0.013$ & $0.150 \pm 0.017$ & $0.154 \pm 0.012$ \\
\hline Glycerol 1-octadecanoate & $0.158 \pm 0.008$ & $0.123 \pm 0.031$ & $0.117 \pm 0.013$ \\
\hline Glycerol-2-phosphate & $0.536 \pm 0.185$ & $1.082 \pm 0.265$ & $1.099 \pm 0.337$ \\
\hline Glycerol-3-phosphate & $8.921 \pm 2.998$ & $0.973 \pm 0.378$ & $2.931 \pm 1.476$ \\
\hline Glycine & $19.031 \pm 1.067$ & $12.358 \pm 1.110$ & $17.139 \pm 1.135$ \\
\hline Guanine & $4.121 \pm 0.719$ & $0.327 \pm 0.182$ & $1.221 \pm 0.672$ \\
\hline Heptadecanoic acid (C17:0) & $0.109 \pm 0.005$ & $0.090 \pm 0.009$ & $0.101 \pm 0.009$ \\
\hline Hexadecanol & $0.662 \pm 0.119$ & $1.098 \pm 0.197$ & $0.950 \pm 0.223$ \\
\hline Hexadecatrienoic acid (C16:3) & $0.121 \pm 0.006$ & $0.057 \pm 0.003$ & $0.089 \pm 0.015$ \\
\hline Inosine & $21.814 \pm 2.182$ & $0.958 \pm 0.586$ & $5.066 \pm 2.842$ \\
\hline
\end{tabular}




\begin{tabular}{|c|c|c|c|}
\hline chiro-Inositol & $0.221 \pm 0.158$ & $0.062 \pm 0.009$ & $0.072 \pm 0.007$ \\
\hline myo-Inositol & $1.845 \pm 0.168$ & $0.719 \pm 0.123$ & $1.333 \pm 0.304$ \\
\hline scyllo-Inositol & $1.038 \pm 0.057$ & $0.479 \pm 0.028$ & $0.766 \pm 0.122$ \\
\hline myo-Inositol-1-phosphate & $2.931 \pm 0.077$ & $0.432 \pm 0.091$ & $1.347 \pm 0.672$ \\
\hline Isoleucine & $21.076 \pm 3.244$ & $7.341 \pm 0.770$ & $11.903 \pm 0.683$ \\
\hline Lactose & $0.065 \pm 0.008$ & $0.058 \pm 0.006$ & $0.092+0.028$ \\
\hline Leucine & $48.012 \pm 4.781$ & $21.866 \pm 1.748$ & $39.203 \pm 4.511$ \\
\hline Linoleic acid (C18:2) & $0.769 \pm 0.073$ & $0.257 \pm 0.020$ & $0.747 \pm 0.080$ \\
\hline Linolenic acid (C18:3) & $1.235 \pm 0.122$ & $0.392 \pm 0.037$ & $1.142 \pm 0.098$ \\
\hline Lysine & $6.385 \pm 1.044$ & $2.557 \pm 0.360$ & $5.028 \pm 1.287$ \\
\hline Malic acid & $0.165 \pm 0.019$ & $0.033 \pm 0.004$ & $0.049 \pm 0.007$ \\
\hline Mannitol & $0.037 \pm 0.025$ & $0.029 \pm 0.009$ & $0.014 \pm 0.003$ \\
\hline Mannose & $0.188 \pm 0.037$ & $0.961 \pm 0.786$ & $0.416 \pm 0.248$ \\
\hline Mannose-6-phosphate & $0.051 \pm 0.012$ & $0.006 \pm 0.001$ & $0.012 \pm 0.004$ \\
\hline Methionine & $9.518 \pm 0.922$ & $2.802 \pm 0.160$ & $5.785 \pm 1.062$ \\
\hline Myristic acid (C14:0) & $0.213 \pm 0.029$ & $0.282 \pm 0.045$ & $0.249 \pm 0.065$ \\
\hline N,N-Dimethyllysine & $1.677 \pm 0.723$ & $0.688 \pm 0.357$ & $1.121 \pm 0.575$ \\
\hline Nonadecanoic acid (C19:0) & $0.039 \pm 0.012$ & $0.035+0.009$ & $0.049+0.019$ \\
\hline Oleic acid (C18:1) & $1.009 \pm 0.106$ & $0.457 \pm 0.039$ & $1.112 \pm 0.085$ \\
\hline Palmitoleic acid (C16:1) & $0.637 \pm 0.052$ & $0.245 \pm 0.025$ & $0.561 \pm 0.041$ \\
\hline Pantothenic acid & $0.276 \pm 0.091$ & $0.079 \pm 0.014$ & $0.533 \pm 0.303$ \\
\hline Pentadecanoic acid (C15:0) & $0.047 \pm 0.007$ & $0.042 \pm 0.003$ & $0.047 \pm 0.002$ \\
\hline Phenylalanine & $14.593 \pm 1.203$ & $6.997 \pm 0.546$ & $12.709 \pm 2.842$ \\
\hline Phosphonic acid & $1.338 \pm 0.343$ & $0.111 \pm 0.041$ & $0.345 \pm 0.164$ \\
\hline Phosphoric acid & $13.904 \pm 4.552$ & $3.181 \pm 0.630$ & $5.105 \pm 1.819$ \\
\hline Pinitol & $0.045 \pm 0.012$ & $0.031 \pm 0.005$ & $0.026 \pm 0.002$ \\
\hline Proline & $26.157 \pm 2.7752$ & $9.815 \pm 0.724$ & $14.433 \pm 0.432$ \\
\hline Putrescine & $0.617 \pm 0.143$ & $1.004+0.138$ & $1.113 \pm 0.258$ \\
\hline Pyroglutamic acid & $49.432 \pm 4.065$ & $10.127 \pm 1.919$ & $18.486 \pm 5.693$ \\
\hline Ribitol & $13.034 \pm 0.410$ & $16.488 \pm 1.739$ & $21.560 \pm 1.126$ \\
\hline Ribose & $21.212+1.877$ & $2.795 \pm 1.003$ & $7.665 \pm 2.977$ \\
\hline Serine & $8.577 \pm 0.676$ & $4.523 \pm 0.514$ & $7.352 \pm 0.870$ \\
\hline Succinic acid & $0.655 \pm 0.097$ & $0.095 \pm 0.008$ & $0.172 \pm 0.019$ \\
\hline Sucrose & $0.095 \pm 0.010$ & $0.083 \pm 0.016$ & $0.187 \pm 0.093$ \\
\hline Threonine & $6.320 \pm 0.282$ & $3.117 \pm 0.175$ & $4.697 \pm 0.417$ \\
\hline Thymidine & $0.241 \pm 0.065$ & $0.071 \pm 0.016$ & $0.366 \pm 0.206$ \\
\hline Thymine & $0.394 \pm 0.066$ & $0.096 \pm 0.017$ & $0.147 \pm 0.073$ \\
\hline Triethanolamine & $0.070 \pm 0.006$ & $0.107 \pm 0.012$ & $0.134+0.021$ \\
\hline Tryptophan & $13.665 \pm 1.661$ & $3.409 \pm 0.382$ & $7.644 \pm 2.305$ \\
\hline Tyramine & $13.699 \pm 2.879$ & $2.264 \pm 0.749$ & $10.431+1.898$ \\
\hline Tyrosine & $32.954 \pm 5.578$ & $6.487 \pm 1.171$ & $17.847 \pm 5.496$ \\
\hline Uric acid & $0.703 \pm 0.170$ & $0.044 \pm 0.017$ & $0.257 \pm 0.141$ \\
\hline Uridine & $2.987 \pm 0.561$ & $0.034+0.008$ & $0.225 \pm 0.150$ \\
\hline Valine & $26.608 \pm 3.386$ & $10.491 \pm 0.996$ & $16.172 \pm 0.675$ \\
\hline Xylulose & $0.043 \pm 0.015$ & $0.045 \pm 0.011$ & $0.083 \pm 0.042$ \\
\hline
\end{tabular}


Supplementary Table S3.3: Fold change in mean relative metabolite abundance in the Aiptasia host tissues of heterologous D1a-colonised against a homologous B1-colonised (D1a/B1). $\log _{2}$ fold change (FC) of the D1a mean divided by the B1 mean abundance. Significance value is Students $t$-test between D1a and B1 relative metabolite abundance $(p<$ $0.05 ; n=5)$.

\begin{tabular}{|c|c|c|c|}
\hline Metabolite & Absolute FC & $\log 2(\mathrm{FC})$ & $p$ value \\
\hline 1-Monooleoylglycerol & 1.866 & 0.9 & \\
\hline Adenosine & 2.263 & 1.178 & \\
\hline Adipic acid & 1.015 & 0.022 & \\
\hline Alanine & 1.435 & 0.521 & \\
\hline Alanine, beta & 1.415 & 0.501 & \\
\hline Arachidonic acid (C20:4) & 1.462 & 0.548 & \\
\hline Arachidyl alcohol & 1.407 & 0.493 & \\
\hline Aspartic acid & 2.077 & 1.054 & \\
\hline Butyric acid & 3.382 & 1.758 & $<0.001$ \\
\hline Campesterol & 1.839 & 0.879 & \\
\hline Cholesterol & 1.334 & 0.416 & \\
\hline Citric acid & 1.323 & 0.404 & \\
\hline Cysteine & 2.027 & 1.02 & \\
\hline Cytosine & 2.293 & 1.197 & \\
\hline Docosahexaenoic acid (C22:6n-3) & 1.434 & 0.52 & \\
\hline Eicosanoic acid (C20:0) & 0.803 & -0.317 & \\
\hline Eicosapentaenoic acid (C20:5) & 1.819 & 0.863 & \\
\hline Eicosatrienoic acid (C20:3) & 2.001 & 1.001 & 0.081 \\
\hline Erythrose & 0.863 & -0.213 & \\
\hline Ethanolamine & 1.577 & 0.657 & \\
\hline Fructose & 1.687 & 0.755 & \\
\hline Fructose-6-phosphate & 3.126 & 1.644 & \\
\hline Galactose & 1.107 & 0.146 & \\
\hline Glucopyranose & 1.172 & 0.229 & \\
\hline Glucosamine & 2.501 & 1.322 & 0.023 \\
\hline Glucose & 1.047 & 0.066 & \\
\hline Glutamate & 0.894 & -0.162 & \\
\hline Glycerate 3-phosphate & 2.189 & 1.13 & \\
\hline Glyceric acid & 1.481 & 0.566 & \\
\hline Glycerol & 1.409 & 0.495 & \\
\hline Glycerol 1-hexadecanoate & 1.027 & 0.039 & \\
\hline Glycerol 1-octadecanoate & 1.903 & 0.929 & \\
\hline Glycerol-2-phosphate & 1.016 & 0.023 & \\
\hline Glycerol-3-phosphate & 3.011 & 1.59 & \\
\hline Glycine & 1.485 & 0.57 & \\
\hline Guanine & 3.733 & 1.9 & \\
\hline Heptadecanoic acid (C17:0) & 1.12 & 0.164 & \\
\hline Hexadecanol & 0.865 & -0.209 & \\
\hline Hexadecatrienoic acid (C16:3) & 1.55 & 0.632 & \\
\hline Inosine & 5.287 & 2.402 & \\
\hline chiro-Inositol & 1.164 & 0.219 & \\
\hline
\end{tabular}




\begin{tabular}{|c|c|c|c|}
\hline myo-Inositol & 1.854 & 0.891 & \\
\hline scyllo-Inositol & 1.599 & 0.677 & \\
\hline myo-Inositol-1-phosphate & 3.115 & 1.639 & \\
\hline Isoleucine & 1.621 & 0.697 & \\
\hline Lactose & 1.584 & 0.664 & \\
\hline Leucine & 1.793 & 0.842 & \\
\hline Linoleic acid (C18:2) & 2.906 & 1.539 & $<0.001$ \\
\hline Linolenic acid (C18:3) & 2.913 & 1.543 & $<0.001$ \\
\hline Lysine & 2.911 & 1.541 & \\
\hline Malic acid & 1.462 & 0.548 & \\
\hline Mannitol & 0.469 & -1.091 & \\
\hline Mannose & 0.433 & -1.206 & \\
\hline Mannose-6-phosphate & 2.233 & 1.159 & \\
\hline Methionine & 2.065 & 1.046 & 0.008 \\
\hline Myristic acid (C14:0) & 0.883 & -0.18 & \\
\hline N,N-Dimethyllysine & 1.63 & 0.705 & \\
\hline Nonadecanoic acid (C19:0) & 1.405 & 0.49 & \\
\hline Oleic acid (C18:1) & 2.433 & 1.283 & $<0.001$ \\
\hline Palmitoleic acid (C16:1) & 2.29 & 1.195 & $<0.001$ \\
\hline Pantothenic acid & 6.733 & 2.751 & \\
\hline Pentadecanoic acid (C15:0) & 1.132 & 0.178 & \\
\hline Phenylalanine & 1.816 & 0.861 & \\
\hline Phosphonic acid & 3.119 & 1.641 & \\
\hline Phosphoric acid & 1.605 & 0.682 & \\
\hline Pinitol & 0.845 & -0.244 & \\
\hline Proline & 1.471 & 0.556 & \\
\hline Putrescine & 1.109 & 0.149 & \\
\hline Pyroglutamic acid & 1.826 & 0.868 & \\
\hline Ribitol & 1.308 & 0.387 & \\
\hline Ribose & 2.742 & 1.455 & \\
\hline Serine & 1.625 & 0.701 & \\
\hline Succinic acid & 1.803 & 0.85 & \\
\hline Sucrose & 2.256 & 1.173 & \\
\hline Threonine & 1.507 & 0.592 & \\
\hline Thymidine & 5.141 & 2.362 & \\
\hline Thymine & 1.53 & 0.613 & \\
\hline Triethanolamine & 1.249 & 0.321 & \\
\hline Tryptophan & 2.242 & 1.165 & \\
\hline Tyramine & 4.607 & 2.204 & 0.004 \\
\hline Tyrosine & 2.751 & 1.46 & 0.045 \\
\hline Uric acid & 5.798 & 2.536 & \\
\hline Uridine & 6.659 & 2.735 & \\
\hline Valine & 1.542 & 0.624 & \\
\hline Xylulose & 1.849 & 0.887 & \\
\hline
\end{tabular}


Supplemental Table S4.1. Summary of the mean comparative data for the metabolites identified in the host tissues of Aiptasia when aposymbiotic, or colonised with homologous (type B1), or heterologous (type D1a) Symbiodinium. Data are mean relative abundance \pm S.E.M. $(n=6)$.

\begin{tabular}{|c|c|c|c|c|c|c|}
\hline Metabolite & Aposymbiotic & \pm S.E.M. & $\begin{array}{r}\text { Homologous } \\
\text { type B1 }\end{array}$ & + S.E.M. & $\begin{array}{r}\text { Heterologous } \\
\text { type D1a }\end{array}$ & + S.E.M. \\
\hline Adenosine & 2.781 & \pm 0.252 & 5.017 & \pm 0.543 & 3.682 & \pm 0.192 \\
\hline Alanine & 45.682 & \pm 5.596 & 20.081 & \pm 1.378 & 14.483 & \pm 0.969 \\
\hline Arachidic acid (C20:0) & 0.505 & \pm 0.060 & 0.341 & \pm 0.069 & 0.184 & \pm 0.018 \\
\hline Arginine & 0.015 & \pm 0.002 & 0.027 & \pm 0.006 & 0.053 & \pm 0.014 \\
\hline Aspartic acid & 15.011 & \pm 1.787 & 4.042 & \pm 1.050 & 4.104 & \pm 0.938 \\
\hline Benzoic acid & 0.986 & \pm 0.057 & 1.083 & \pm 0.067 & 0.915 & \pm 0.056 \\
\hline beta-Alanine & 11.147 & \pm 1.902 & 12.047 & \pm 1.017 & 8.691 & \pm 1.564 \\
\hline Butyric acid & 1.230 & \pm 0.199 & 4.713 & \pm 0.536 & 3.091 & \pm 0.650 \\
\hline Campesterol & 0.778 & \pm 0.071 & 0.446 & \pm 0.067 & 0.500 & \pm 0.077 \\
\hline Cholesterol & 29.384 & \pm 2.797 & 19.397 & \pm 2.925 & 18.924 & \pm 1.684 \\
\hline Citric acid & 2.208 & \pm 0.385 & 0.962 & \pm 0.140 & 1.015 & \pm 0.268 \\
\hline Cytosine & 0.289 & \pm 0.038 & 0.243 & \pm 0.029 & 0.247 & \pm 0.022 \\
\hline $\begin{array}{l}\text { Docosahexaenoic acid } \\
\text { (C22:6n-3) }\end{array}$ & 0.591 & \pm 0.065 & 7.653 & \pm 1.525 & 1.002 & \pm 0.168 \\
\hline $\begin{array}{l}\text { Dodecanoic acid } \\
\text { (C12:0) }\end{array}$ & 0.144 & \pm 0.018 & 0.898 & \pm 0.044 & 0.178 & \pm 0.019 \\
\hline Erucic acid (C22:1) & 4.071 & \pm 0.400 & 2.930 & \pm 0.561 & 2.113 & \pm 0.170 \\
\hline Ethanolamine & 1.542 & \pm 0.198 & 2.822 & \pm 0.170 & 1.826 & \pm 0.218 \\
\hline Fructose & 1.082 & \pm 0.186 & 1.265 & \pm 0.112 & 0.995 & \pm 0.090 \\
\hline Fumaric acid & 0.465 & \pm 0.069 & 0.210 & \pm 0.027 & 0.094 & \pm 0.023 \\
\hline Galactose & 0.693 & \pm 0.084 & 4.733 & \pm 0.373 & 2.538 & \pm 2.052 \\
\hline Gluconic acid & 0.052 & \pm 0.007 & 0.347 & \pm 0.056 & 0.082 & \pm 0.010 \\
\hline Glucopyranose & 0.381 & \pm 0.075 & 6.100 & \pm 2.307 & 0.140 & \pm 0.032 \\
\hline Glucose & 1.316 & \pm 0.385 & 148.229 & \pm 7.283 & 18.757 & +2.685 \\
\hline Glutamate & 50.933 & \pm 4.784 & 18.986 & \pm 3.090 & 18.470 & \pm 2.638 \\
\hline Glutamine & 0.060 & \pm 0.007 & 0.411 & \pm 0.065 & 0.055 & \pm 0.004 \\
\hline Glyceric acid & 1.824 & \pm 0.153 & 2.527 & \pm 0.164 & 1.725 & \pm 0.200 \\
\hline Glycerol & 5.210 & \pm 0.369 & 41.457 & \pm 2.293 & 8.863 & \pm 0.772 \\
\hline Glycerol-2-P & 5.942 & \pm 0.787 & 3.954 & \pm 1.247 & 2.596 & \pm 0.426 \\
\hline Glycerol-3-P & 47.157 & \pm 1.285 & 27.031 & \pm 6.548 & 25.235 & \pm 3.550 \\
\hline Glycine & 49.258 & \pm 4.976 & 32.589 & \pm 3.648 & 19.435 & \pm 2.331 \\
\hline Glycolic acid & 1.060 & \pm 0.117 & 0.495 & \pm 0.032 & 0.388 & \pm 0.037 \\
\hline Guanine & 90.490 & \pm 5.289 & 63.531 & \pm 10.542 & 94.621 & \pm 13.601 \\
\hline Guanosine & 1.797 & \pm 0.225 & 0.726 & \pm 0.110 & 0.822 & \pm 0.156 \\
\hline Heptadecan-1-ol & 18.927 & +8.329 & 32.605 & \pm 7.629 & 7.465 & \pm 0.927 \\
\hline Hexadecan-1-ol & 8.836 & \pm 1.581 & 12.079 & \pm 0.767 & 7.585 & \pm 0.949 \\
\hline Hexanoic acid (C6:0) & 0.283 & \pm 0.048 & 0.425 & \pm 0.047 & 0.262 & \pm 0.059 \\
\hline Isoleucine & 22.757 & \pm 2.803 & 23.127 & \pm 2.357 & 17.389 & \pm 2.677 \\
\hline Lactic acid & 2.314 & \pm 0.519 & 2.704 & \pm 0.489 & 1.836 & \pm 0.155 \\
\hline Linoleic acid (C18:2) & 6.239 & \pm 0.249 & 3.015 & \pm 0.398 & 3.785 & \pm 0.348 \\
\hline Linolenic acid (C18:6) & 2.991 & \pm 0.140 & 0.848 & \pm 0.154 & 1.640 & \pm 0.159 \\
\hline Lysine & 8.186 & \pm 1.535 & 5.999 & \pm 0.893 & 7.567 & \pm 1.622 \\
\hline
\end{tabular}




\begin{tabular}{|c|c|c|c|c|c|c|}
\hline Malonic acid & 1.084 & \pm 0.120 & 1.689 & \pm 0.670 & 0.600 & \pm 0.116 \\
\hline Maltose & 0.819 & \pm 0.626 & 40.879 & \pm 4.866 & 0.860 & \pm 0.144 \\
\hline Mannitol & 0.040 & \pm 0.007 & 3.039 & \pm 0.185 & 0.209 & \pm 0.029 \\
\hline Margaric acid (C17:0) & 1.296 & \pm 0.158 & 1.012 & \pm 0.116 & 0.789 & \pm 0.101 \\
\hline Methionine & 2.391 & \pm 0.493 & 0.876 & \pm 0.478 & 1.613 & \pm 0.616 \\
\hline myo-Inositol & 4.187 & \pm 0.343 & 6.137 & \pm 0.755 & 1.772 & \pm 0.177 \\
\hline myo-Inositol-1-P & 1.677 & \pm 0.142 & 1.238 & \pm 0.294 & 1.484 & \pm 0.295 \\
\hline myo-Inositol-2-P & 0.198 & \pm 0.010 & 0.093 & \pm 0.023 & 0.155 & \pm 0.048 \\
\hline Myristic acid (C14:0) & 0.740 & \pm 0.101 & 4.611 & \pm 0.341 & 0.978 & \pm 0.116 \\
\hline Nicotinic acid & 0.488 & \pm 0.104 & 1.167 & \pm 0.090 & 0.433 & \pm 0.052 \\
\hline Nonanoic acid (C9:0) & 0.200 & \pm 0.023 & 0.248 & \pm 0.043 & 0.210 & \pm 0.043 \\
\hline Octadecan-1-ol & 3.329 & \pm 0.518 & 3.533 & \pm 0.284 & 2.757 & \pm 0.405 \\
\hline Octanoic acid (C8:0) & 0.128 & \pm 0.018 & 0.134 & \pm 0.009 & 0.107 & \pm 0.012 \\
\hline Oleic acid (C18:1) & 14.259 & \pm 0.697 & 12.051 & \pm 1.226 & 9.812 & \pm 0.776 \\
\hline Ornithine & 2.506 & \pm 0.449 & 0.499 & \pm 0.092 & 0.803 & \pm 0.134 \\
\hline $\begin{array}{l}\text { Palmitic acid (C16:0) } \\
\text { Palmitoleic acid }\end{array}$ & 27.183 & \pm 6.934 & 40.405 & \pm 5.028 & 14.490 & \pm 1.764 \\
\hline $\begin{array}{l}\text { (C16:1) } \\
\text { Pentadecanoic acid }\end{array}$ & 2.910 & \pm 0.255 & 2.867 & \pm 0.281 & 2.664 & \pm 0.284 \\
\hline$(\mathrm{C} 15: 0)$ & 6.357 & \pm 2.062 & 9.769 & \pm 0.751 & 6.748 & \pm 0.862 \\
\hline Phenylalanine & 26.916 & \pm 2.554 & 26.677 & \pm 2.023 & 25.338 & \pm 2.012 \\
\hline Phosphoric acid & 86.768 & \pm 9.309 & 27.019 & \pm 5.393 & 21.808 & \pm 3.561 \\
\hline Picolinic acid & 2.759 & \pm 0.393 & 0.469 & \pm 0.144 & 0.431 & \pm 0.038 \\
\hline Putrescine & 1.668 & \pm 0.251 & 1.839 & \pm 0.451 & 4.424 & \pm 0.765 \\
\hline Pyroglutamic acid & 105.490 & \pm 6.877 & 29.914 & \pm 5.214 & 29.168 & \pm 4.757 \\
\hline Ribose & 0.044 & \pm 0.004 & 1.743 & \pm 0.380 & 0.175 & \pm 0.017 \\
\hline scyllo-Inositol & 1.714 & \pm 0.153 & 13.796 & \pm 2.257 & 0.925 & \pm 0.111 \\
\hline Serine & 1.071 & \pm 0.416 & 0.327 & \pm 0.028 & 1.186 & \pm 0.689 \\
\hline Stearic acid (C18:0) & 26.498 & \pm 7.163 & 14.971 & \pm 2.412 & 10.429 & \pm 1.219 \\
\hline Succinic acid & 1.635 & \pm 0.153 & 1.014 & \pm 0.149 & 0.433 & \pm 0.053 \\
\hline Sucrose & 0.334 & \pm 0.063 & 12.760 & \pm 1.254 & 11.303 & \pm 1.152 \\
\hline Threonic acid & 0.207 & \pm 0.012 & 2.185 & \pm 0.277 & 0.535 & \pm 0.045 \\
\hline Threonine & 10.498 & \pm 1.175 & 7.435 & \pm 0.815 & 5.301 & \pm 0.645 \\
\hline Tryptophan & 9.486 & \pm 1.319 & 5.435 & \pm 0.665 & 4.243 & \pm 0.293 \\
\hline Tyramine & 11.931 & \pm 2.850 & 15.394 & \pm 3.851 & 11.509 & \pm 3.805 \\
\hline Tyrosine & 72.610 & \pm 12.065 & 72.441 & \pm 5.465 & 52.650 & \pm 3.061 \\
\hline Unknown_18.95 & 0.218 & \pm 0.112 & 1.928 & \pm 0.424 & 0.147 & \pm 0.046 \\
\hline Unknown_19.53 & 1.511 & \pm 0.104 & 0.507 & \pm 0.087 & 0.850 & \pm 0.224 \\
\hline Unknown_22.54 & 0.514 & \pm 0.030 & 0.775 & \pm 0.150 & 0.805 & \pm 0.099 \\
\hline Uracil & 0.881 & \pm 0.149 & 0.202 & \pm 0.042 & 0.121 & \pm 0.023 \\
\hline Urea & 0.530 & \pm 0.041 & 3.945 & \pm 0.624 & 3.268 & \pm 0.624 \\
\hline Valine & 26.342 & \pm 3.059 & 28.462 & \pm 2.353 & 18.704 & \pm 2.828 \\
\hline Xylose & 0.039 & +0.014 & 4.659 & +0.917 & 0.074 & +0.009 \\
\hline
\end{tabular}


Supplemental Table S4.2. Summarised NTFD output for Aiptasia when aposymbiotic, or colonised with homologous (type B1), or heterologous (type D1a) Symbiodinium. Data are the unlabelled $\left(\mathrm{M}_{0}\right)$ and labelled $\left(1-\mathrm{M}_{0}\right)$ proportions detected. See text for calculations $(n=6)$.

\begin{tabular}{|c|c|c|c|c|c|c|}
\hline \multirow[b]{2}{*}{ Metabolite } & \multicolumn{2}{|c|}{ Aposymbiotic } & \multicolumn{2}{|c|}{ Homologous type B1 } & \multicolumn{2}{|c|}{ Heterologous type D1a } \\
\hline & $\mathbf{M}_{0}$ & $1-M_{0}$ & $\mathbf{M}_{0}$ & $1-M_{0}$ & $\mathbf{M}_{0}$ & $1-M_{0}$ \\
\hline Arginine & 0 & 0 & 0.870 & 0.130 & 0.884 & 0.116 \\
\hline Aspartic acid & 0.917 & 0.093 & 0.816 & 0.184 & 0 & 0 \\
\hline Fructose & 0 & 0 & 0.782 & 0.218 & 0 & 0 \\
\hline Galactosylglycerol & 0 & 0 & 0.902 & 0.098 & 0 & 0 \\
\hline Gluconic acid & 0 & 0 & 0.819 & 0.181 & 0 & 0 \\
\hline Glucose & 0 & 0 & 0.774 & 0.226 & 0.610 & 0.390 \\
\hline Glutamate & 0 & 0 & 0.833 & 0.167 & 0.929 & 0.071 \\
\hline Glutamine & 0 & 0 & 0 & 0 & 0.943 & 0.057 \\
\hline Glyceric acid & 0 & 0 & 0.896 & 0.104 & 0 & 0 \\
\hline Guanosine & 0 & 0 & 0.944 & 0.056 & 0 & 0 \\
\hline myo-Inositol & 0 & 0 & 0.926 & 0.074 & 0 & 0 \\
\hline scyllo-Inositol & 0 & 0 & 0.665 & 0.335 & 0.880 & 0.120 \\
\hline Leucine & 0 & 0 & 0.882 & 0.118 & 0.904 & 0.096 \\
\hline Linoleic acid (C18:2) & 0 & 0 & 0.939 & 0.061 & 0 & 0 \\
\hline Maltose & 0 & 0 & 0.875 & 0.125 & 0 & 0 \\
\hline Mannitol & 0 & 0 & 0.765 & 0.235 & 0.606 & 0.394 \\
\hline Myristic acid (C14:0) & 0 & 0 & 0.918 & 0.082 & 0.817 & 0.183 \\
\hline Octadecadienoic acid & 0 & 0 & 0.941 & 0.060 & 0 & 0 \\
\hline Ornithine & 0 & 0 & 0 & 0 & 0.932 & 0.068 \\
\hline Palmitic acid (C16:0) & 0 & 0 & 0.903 & 0.097 & 0 & 0 \\
\hline $\begin{array}{l}\text { Palmitoleic acid } \\
\text { (C16:1) }\end{array}$ & 0 & 0 & 0.767 & 0.233 & 0.936 & 0.064 \\
\hline Pyroglutamic acid & 0 & 0 & 0.864 & 0.136 & 0 & 0 \\
\hline Serine & 0 & 0 & 0.952 & 0.048 & 0 & 0 \\
\hline Stearic acid (C18:0) & 0 & 0 & 0.865 & 0.135 & 0.824 & 0.176 \\
\hline Sucrose & 0 & 0 & 0.659 & 0.341 & 0 & 0 \\
\hline Threonic acid & 0 & 0 & 0.877 & 0.123 & 0 & 0 \\
\hline Tyramine & 0 & 0 & 0 & 0 & 0.936 & 0.064 \\
\hline Unknown_19.53 & 0 & 0 & 0.726 & 0.274 & 0 & 0 \\
\hline Unknown_22.54 & 0 & 0 & 0.911 & 0.089 & 0 & 0 \\
\hline Unknown_28.8 & 0 & 0 & 0.854 & 0.146 & 0 & 0 \\
\hline Xylose & 0 & 0 & 0.949 & 0.051 & 0 & 0 \\
\hline
\end{tabular}


Supplemental Table S4.3. Summary of the test for significance between the net relative abundance of the unlabelled $\left(M_{0}\right)$ and labelled $\left(M_{i}\right)$ fractions of the metabolites identified as enriched in both homologous B1-colonised and heterologous D1a-colonised Aiptasia host tissues. Two-tailed $t$-test statistic $(t)$, degrees of freedom (df), significance value ( $p$ value) are provided. $*=p<0.05, * *=p<0.001$.

\begin{tabular}{|c|c|c|c|c|c|}
\hline Metabolite & Fraction & $t$ & df & \multicolumn{2}{|c|}{$p$ value } \\
\hline Arginine & M0 & 0.472 & 10 & 0.647 & \\
\hline Glucose & M0 & & 10 & 0.002 & $*$ \\
\hline Glutamate & M0 & -0.606 & 10 & 0.558 & \\
\hline scyllo-Inositol & M0 & 11.318 & 10 & $<0.001$ & $* *$ \\
\hline Leucine & M0 & & 10 & 0.24 & \\
\hline Mannitol & M0 & 13.185 & 10 & $<0.001$ & $* *$ \\
\hline Stearic acid (C18:0) & M0 & 1.992 & 10 & 0.074 & \\
\hline Palmitoleic acid (C16:1) & M0 & -1.005 & 10 & 0.339 & \\
\hline Myristic acid (C14:0) & M0 & 11.601 & 10 & $<0.001$ & $* *$ \\
\hline Arginine & $\mathrm{Mi}$ & 1.193 & 10 & 0.26 & \\
\hline Glucose & $\mathrm{Mi}$ & & 10 & 0.002 & $*$ \\
\hline Glutamate & Mi & 3.75 & 10 & 0.004 & $*$ \\
\hline scyllo-Inositol & $\mathrm{Mi}$ & 17.02 & 10 & $<0.001$ & $* *$ \\
\hline Leucine & $\mathrm{Mi}$ & & 10 & 0.937 & \\
\hline Mannitol & $\mathrm{Mi}$ & 9.665 & 10 & $<0.001$ & $* *$ \\
\hline Stearic acid (C18:0) & $\mathrm{Mi}$ & 0.261 & 10 & 0.799 & \\
\hline Palmitoleic acid (C16:1) & $\mathrm{Mi}$ & 9.256 & 10 & $<0.001$ & $* *$ \\
\hline Myristic acid (C14:0) & Mi & 5.013 & 10 & $<0.001$ & $* *$ \\
\hline
\end{tabular}


Supplemental Table S4.4. Summary of the test for significance between the relative abundance of unlabelled $\left(M_{0}\right)$ and labelled $\left(M_{i}\right)$ fractions per symbiont cell of the metabolites identified as enriched in both homologous B1-colonised and heterologous D1acolonised Aiptasia host tissues. Two-tailed $t$-test statistic $(t)$, degrees of freedom (df), significance value ( $p$ value) are provided. $*=p<0.05, * *=p<0.001$. Fold difference is difference in relative mean abundance per symbiont cell between B1 and D1a

\begin{tabular}{|c|c|c|c|c|c|c|}
\hline Metabolite & Fraction & $t$ & df & $p$ value & & $\begin{array}{l}\text { Fold difference } \\
\text { (B1/D1a) }\end{array}$ \\
\hline Arginine & M0 & -2.084 & 10 & 0.064 & & \\
\hline Glucose & M0 & 5.533 & 5.177 & 0.002 & $*$ & $\mathrm{x} 7.2$ \\
\hline Glutamate & M0 & -2.071 & 10 & 0.065 & & \\
\hline scyllo-Inositol & M0 & 8.602 & 10 & 0 & $* *$ & $x 6.7$ \\
\hline Leucine & M0 & -0.678 & 10 & 0.513 & & \\
\hline Mannitol & M0 & 7.151 & 5.297 & $<0.001$ & $* *$ & $x 6.6$ \\
\hline Stearic acid (C18:0) & M0 & -0.02 & 10 & 0.984 & & \\
\hline Palmitoleic acid (C16:1) & M0 & - & - & 0.24 & & \\
\hline Myristic acid (C14:0) & M0 & - & - & 0.002 & $*$ & $\times 3.5$ \\
\hline Arginine & $\mathrm{Mi}$ & 1.301 & 8.385 & 0.228 & & \\
\hline Glucose & $\mathrm{Mi}$ & 4.245 & 10 & 0.002 & $*$ & $\mathrm{x} 3.2$ \\
\hline Glutamate & $\mathrm{Mi}$ & 1.093 & 10 & 0.3 & & \\
\hline scyllo-Inositol & $\mathrm{Mi}$ & 10.048 & 10 & $<0.001$ & $* *$ & $\mathrm{x} 24.2$ \\
\hline Leucine & Mi & -0.366 & 10 & 0.722 & & \\
\hline Mannitol & $\mathrm{Mi}$ & 5.332 & 10 & $<0.001$ & * & $\mathrm{x} 3$ \\
\hline Stearic acid (C18:0) & $\mathrm{Mi}$ & -1.657 & 10 & 0.128 & & \\
\hline Palmitoleic acid (C16:1) & $\mathrm{Mi}$ & - & - & 0.002 & $*$ & $\mathrm{x} 7$ \\
\hline Myristic acid (C14:0) & $\mathrm{Mi}$ & - & - & 0.24 & & \\
\hline
\end{tabular}


Supplemental Table S5.1. Mean quantitative data of the metabolites identified in the host tissues of Montipora capitata grouped according to the Symbiodinium community composition Data are mean relative abundance \pm S.E.M. Labels indicate Symbiodinium composition: $\mathrm{C}=$ clade $\mathrm{C}$-only $(n=20)$; $\mathrm{Cd}=$ clade $\mathrm{C}$ dominant with less abundant clade $\mathrm{D}(n$ $=6) ; \mathrm{Dc}=$ clade $\mathrm{D}$ dominant with less abundant clade $\mathrm{C}(n=11) ; \mathrm{D}=$ clade $\mathrm{D}$-only $(n=3)$.

\begin{tabular}{|c|c|c|c|c|c|c|c|c|c|}
\hline Metabolite & C & \pm & S.E.M & Cd & \pm S.E.M & Dc & \pm S.E.M & $\underline{\mathbf{D}}$ & \pm S.E.M \\
\hline 1-Hexadecanol & $6.92^{-05}$ & \pm & $2.31^{-05}$ & $8.67^{-05}$ & $\pm 3.77^{-05}$ & $4.26^{-05}$ & $\pm 1.46^{-05}$ & $5.97^{-05}$ & $\pm 2.98^{-05}$ \\
\hline 1-Octadecanol & $2.20^{-05}$ & \pm & $7.40^{-06}$ & $3.72^{-05}$ & $\pm 1.43^{-05}$ & $1.41^{-05}$ & $\pm 4.77^{-06}$ & $1.98^{-05}$ & $\pm 9.85^{-06}$ \\
\hline Adenine & $2.50^{-04}$ & \pm & $6.43^{-05}$ & $1.78^{-04}$ & $\pm 5.03^{-05}$ & $1.15^{-04}$ & $\pm 2.91^{-05}$ & $2.10^{-04}$ & $\pm 1.26^{-04}$ \\
\hline Adenosine & $7.82^{-05}$ & \pm & $1.75^{-05}$ & $2.94^{-04}$ & $\pm 2.37^{-04}$ & $2.11^{-04}$ & $\pm 9.29^{-05}$ & $1.83^{-04}$ & $\pm 1.41^{-04}$ \\
\hline Alanine & $1.11^{-04}$ & \pm & $2.80^{-05}$ & $1.20^{-04}$ & $\pm 3.59^{-05}$ & $6.14^{-05}$ & $\pm 1.26^{-05}$ & $1.07^{-04}$ & $\pm 4.80^{-05}$ \\
\hline Benzoic Acid (C4:0) & $1.60^{-05}$ & \pm & $4.92^{-06}$ & $1.76^{-05}$ & $\pm 7.50^{-06}$ & $8.78^{-06}$ & $\pm 3.15^{-06}$ & $8.97^{-06}$ & $\pm 3.22^{-06}$ \\
\hline Campesterol & $1.67^{-05}$ & \pm & $4.47^{-06}$ & $2.62^{-05}$ & $\pm 1.26^{-05}$ & $8.40^{-06}$ & $\pm 2.54^{-06}$ & $1.95^{-05}$ & $\pm 1.38^{-05}$ \\
\hline Cholesterol & $2.34^{-07}$ & \pm & $6.31^{-08}$ & $1.31^{-06}$ & $\pm 8.19^{-07}$ & $1.92^{-07}$ & $\pm 6.01^{-08}$ & $5.05^{-07}$ & $\pm 4.01^{-07}$ \\
\hline Citric acid & $1.52^{-05}$ & \pm & $3.79^{-06}$ & $2.55^{-05}$ & $\pm 9.50^{-06}$ & $8.12^{-06}$ & $\pm 2.31^{-06}$ & $7.22^{-06}$ & $\pm 4.27^{-06}$ \\
\hline Cytosine & $1.04^{-05}$ & \pm & $3.39^{-06}$ & $6.11^{-06}$ & $\pm 1.90^{-06}$ & $5.66^{-06}$ & $\pm 1.26^{-06}$ & $7.57^{-06}$ & $\pm 3.94^{-06}$ \\
\hline Decanoic acid (C10:0) & $5.21^{-06}$ & \pm & $1.33^{-06}$ & $6.77^{-06}$ & $\pm 2.26^{-06}$ & $3.08^{-06}$ & $\pm 1.09^{-06}$ & $2.31^{-05}$ & $\pm 2.09^{-05}$ \\
\hline $\begin{array}{l}\text { Docosahexaenoic acid } \\
\text { (C22:6) }\end{array}$ & $2.04^{-05}$ & \pm & $5.97^{-06}$ & $2.19^{-05}$ & $\pm 1.03^{-05}$ & $1.03^{-05}$ & $\pm 2.68^{-06}$ & $2.60^{-05}$ & $\pm 1.95^{-05}$ \\
\hline $\begin{array}{l}\text { Eicosanoic acid } \\
(\mathrm{C} 20: 0)\end{array}$ & $1.89^{-06}$ & \pm & $4.68^{-07}$ & $5.43^{-06}$ & $\pm 3.39^{-06}$ & $1.02^{-06}$ & $\pm 2.28^{-07}$ & $1.26^{-06}$ & $\pm 6.30^{-07}$ \\
\hline Fructose & $1.64^{-05}$ & \pm & $4.98^{-06}$ & $2.14^{-05}$ & $\pm 7.22^{-06}$ & $1.45^{-05}$ & $\pm 5.79^{-06}$ & $6.71^{-05}$ & $\pm 6.19^{-05}$ \\
\hline Galactose & $8.67^{-05}$ & \pm & $2.79^{-05}$ & $4.81^{-05}$ & $\pm 1.28^{-05}$ & $5.60^{-05}$ & $\pm 1.69^{-05}$ & $9.12^{-05}$ & $\pm 5.35^{-05}$ \\
\hline Glucose & $1.48^{-04}$ & \pm & $4.19^{-05}$ & $1.37^{-04}$ & $\pm 3.18^{-05}$ & $1.37^{-04}$ & $\pm 6.29^{-05}$ & $2.46^{-04}$ & $\pm 1.67^{-04}$ \\
\hline Glutamic acid & $1.49^{-04}$ & \pm & $4.34^{-05}$ & $1.30^{-04}$ & $\pm 6.93^{-05}$ & $5.79^{-05}$ & $\pm 1.27^{-05}$ & $6.51^{-05}$ & $\pm 3.43^{-05}$ \\
\hline Glutamine & $6.96^{-05}$ & \pm & $2.28^{-05}$ & $2.00^{-04}$ & $\pm 1.35^{-04}$ & $4.70^{-05}$ & $\pm 1.55^{-05}$ & $1.01^{-04}$ & $\pm 6.74^{-05}$ \\
\hline Glyceric acid & $5.64^{-05}$ & \pm & $1.20^{-05}$ & $6.12^{-05}$ & $\pm 2.08^{-05}$ & $3.36^{-05}$ & $\pm 1.00^{-05}$ & $2.73^{-05}$ & $\pm 1.38^{-05}$ \\
\hline Glycerol & $7.91^{-05}$ & \pm & $2.18^{-05}$ & $8.65^{-05}$ & $\pm 2.39^{-05}$ & $5.53^{-05}$ & $+2.09^{-05}$ & $1.70^{-04}$ & $\pm 1.34^{-04}$ \\
\hline Glycine & $8.36^{-05}$ & \pm & $1.98^{-05}$ & $8.05^{-05}$ & $\pm 2.50^{-05}$ & $5.07^{-05}$ & $\pm 1.20^{-05}$ & $8.91^{-05}$ & $\pm 4.38^{-05}$ \\
\hline Glycolic acid & $2.99^{-04}$ & \pm & $8.18^{-05}$ & $4.16^{-04}$ & $\pm 1.67^{-04}$ & $1.45^{-04}$ & $\pm 3.59^{-05}$ & $1.16^{-04}$ & $\pm 3.70^{-05}$ \\
\hline Guanine & $7.42^{-06}$ & \pm & $1.96^{-06}$ & $2.77^{-05}$ & $\pm 2.26^{-05}$ & $3.51^{-06}$ & $\pm 7.17^{-07}$ & $5.45^{-06}$ & $\pm 3.45^{-06}$ \\
\hline Guanosine & $4.84^{-06}$ & \pm & $1.26^{-06}$ & $9.38^{-06}$ & $\pm 6.66^{-06}$ & $5.44^{-06}$ & $\pm 2.13^{-06}$ & $2.57^{-06}$ & $\pm 1.17^{-06}$ \\
\hline $\begin{array}{l}\text { Hexadecanoic acid } \\
(\mathrm{C} 16: 0)\end{array}$ & $2.58^{-04}$ & \pm & $7.39^{-05}$ & $7.80^{-04}$ & $\pm 5.36^{-04}$ & $1.25^{-04}$ & $\pm 3.43^{-05}$ & $2.17^{-04}$ & $\pm 1.28^{-04}$ \\
\hline myo-Inositol-2-P & $3.14^{-06}$ & \pm & $6.04^{-07}$ & $2.50^{-06}$ & $\pm 8.03^{-07}$ & $1.58^{-06}$ & $\pm 4.65^{-07}$ & $1.74^{-06}$ & $\pm 8.19^{-07}$ \\
\hline myo-Inositol & $4.11^{-04}$ & \pm & $1.04^{-04}$ & $5.24^{-04}$ & $\pm 1.61^{-04}$ & $2.19^{-04}$ & $\pm 6.26^{-05}$ & $3.87^{-04}$ & $\pm 2.30^{-04}$ \\
\hline scyllo-Inositol & $3.21^{-03}$ & \pm & $9.26^{-04}$ & $3.46^{-03}$ & $\pm 1.06^{-03}$ & $1.69^{-03}$ & $\pm 5.04^{-04}$ & $2.30^{-03}$ & $\pm 9.24^{-04}$ \\
\hline Isoleucine & $5.72^{-05}$ & \pm & $1.50^{-05}$ & $6.06^{-05}$ & $\pm 2.70^{-05}$ & $3.14^{-05}$ & $\pm 5.98^{-06}$ & $4.42^{-05}$ & $\pm 2.16^{-05}$ \\
\hline Lactic acid & $5.06^{-05}$ & \pm & $1.56^{-05}$ & $7.32^{-05}$ & $\pm 4.40^{-05}$ & $2.78^{-05}$ & $\pm 1.06^{-05}$ & $2.91^{-05}$ & $\pm 1.21^{-05}$ \\
\hline Mannose & $4.85^{-04}$ & \pm & $1.48^{-04}$ & $5.53^{-04}$ & $\pm 1.28^{-04}$ & $4.55^{-04}$ & $\pm 1.88^{-04}$ & $8.40^{-04}$ & $\pm 5.37^{-04}$ \\
\hline Myristic acid (C14:0) & $1.74^{-05}$ & \pm & $4.68^{-06}$ & $3.10^{-05}$ & $\pm 1.27^{-05}$ & $1.11^{-05}$ & $\pm 3.06^{-06}$ & $2.63^{-05}$ & $\pm 1.86^{-05}$ \\
\hline Nicotinic acid & $3.50^{-05}$ & \pm & $8.64^{-06}$ & $4.28^{-05}$ & $\pm 1.36^{-05}$ & $2.43^{-05}$ & $\pm 8.28^{-06}$ & $2.02^{-05}$ & $\pm 9.58^{-06}$ \\
\hline Nonanoic acid (C9:0) & $4.63^{-06}$ & \pm & $1.64^{-06}$ & $4.15^{-06}$ & $\pm 1.48^{-06}$ & $2.37^{-06}$ & $\pm 7.74^{-07}$ & $2.98^{-06}$ & $\pm 1.11^{-06}$ \\
\hline Oleic acid (C18:1) & $5.69^{-06}$ & \pm & $1.35^{-06}$ & $8.54^{-06}$ & $\pm 3.29^{-06}$ & $4.03^{-06}$ & $\pm 1.18^{-06}$ & $5.51^{-06}$ & $\pm 2.53^{-06}$ \\
\hline Octanoic acid (C8:0) & $2.33^{-05}$ & \pm & $9.23^{-06}$ & $1.88^{-05}$ & $\pm 8.45^{-06}$ & $1.10^{-05}$ & $\pm 3.06^{-06}$ & $1.17^{-05}$ & $\pm 1.27^{-06}$ \\
\hline $\begin{array}{l}\text { Palmitoleic acid } \\
\text { (C16:1) }\end{array}$ & $2.89^{-06}$ & \pm & $7.81^{-07}$ & $3.66^{-06}$ & $\pm 1.26^{-06}$ & $2.14^{-06}$ & $\pm 6.04^{-07}$ & $7.84^{-06}$ & $\pm 6.26^{-06}$ \\
\hline Phenylalanine & $1.41^{-05}$ & \pm & $5.06^{-06}$ & $8.83^{-06}$ & $\pm 3.21^{-06}$ & $2.90^{-06}$ & $\pm 9.36^{-07}$ & $1.52^{-06}$ & $\pm 1.20^{-06}$ \\
\hline Phosphoric acid & $2.49^{-04}$ & \pm & $6.28^{-05}$ & $3.37^{-04}$ & $\pm 1.23^{-04}$ & $1.21^{-04}$ & $\pm 3.54^{-05}$ & $5.31^{-05}$ & $\pm 2.92^{-05}$ \\
\hline
\end{tabular}




\begin{tabular}{llllll} 
Pyroglutamic acid & $4.38^{-04} \pm 1.13^{-04}$ & $4.78^{-04} \pm 1.84^{-04}$ & $1.92^{-04} \pm 3.28^{-05}$ & $1.67^{-04} \pm 7.93^{-05}$ \\
Ribonic acid & $2.73^{-05} \pm 6.08^{-06}$ & $4.27^{-05} \pm 1.66^{-05}$ & $1.61^{-05} \pm 4.41^{-06}$ & $1.88^{-05} \pm 9.72^{-06}$ \\
Ribose & $5.03^{-05} \pm 1.46^{-05}$ & $5.30^{-05} \pm 1.21^{-05}$ & $4.71^{-05} \pm 2.30^{-05}$ & $7.18^{-05} \pm 5.12^{-05}$ \\
Ribose-5-P & $7.09^{-06} \pm 3.62^{-06}$ & $3.40^{-06} \pm 1.36^{-06}$ & $3.56^{-06} \pm 2.39^{-06}$ & $1.12^{-05} \pm 1.05^{-05}$ \\
Serine & $9.33^{-05} \pm 3.19^{-05}$ & $7.96^{-05} \pm 4.08^{-05}$ & $4.46^{-05} \pm 9.91^{-06}$ & $7.45^{-05} \pm 3.91^{-05}$ \\
Stearic acid (C18:0) & $1.25^{-04} \pm 4.14^{-05}$ & $6.07^{-04} \pm 4.99^{-04}$ & $4.99^{-05} \pm 1.44^{-05}$ & $6.06^{-05} \pm 2.85^{-05}$ \\
Succinic acid & $3.14^{-06} \pm 9.09^{-07}$ & $4.04^{-06} \pm 1.39^{-06}$ & $1.63^{-06} \pm 3.91^{-07}$ & $1.39^{-06} \pm 5.14^{-07}$ \\
Sucrose & $2.47^{-04} \pm 1.54^{-04}$ & $6.89^{-05} \pm 2.68^{-05}$ & $5.08^{-05} \pm 2.28^{-05}$ & $8.95^{-05} \pm 5.40^{-05}$ \\
Threonic acid & $4.73^{-05} \pm 1.09^{-05}$ & $5.00^{-05} \pm 1.70^{-05}$ & $2.16^{-05} \pm 4.72^{-06}$ & $6.60^{-05} \pm 5.04^{-05}$ \\
Threonine & $4.25^{-05} \pm 1.03^{-05}$ & $5.46^{-05} \pm 2.64^{-05}$ & $2.68^{-05} \pm 7.25^{-06}$ & $3.88^{-05} \pm 2.05^{-05}$ \\
Thymine & $4.74^{-05} \pm 1.20^{-05}$ & $2.77^{-05} \pm 1.36^{-05}$ & $3.85^{-05} \pm 1.81^{-05}$ & $2.91^{-05} \pm 1.18^{-05}$ \\
Tyrosine & $3.22^{-06} \pm 8.90^{-07}$ & $4.38^{-06} \pm 3.70^{-06}$ & $1.28^{-06} \pm 4.31^{-07}$ & $2.37^{-06} \pm 1.87^{-06}$ \\
Uracil & $1.85^{-05} \pm 1.03^{-05}$ & $6.15^{-06} \pm 3.73^{-06}$ & $9.63^{-07} \pm 6.39^{-06}$ & $2.80^{-06} \pm 1.58^{-06}$ \\
Valine & $1.49^{-04} \pm 4.07^{-05}$ & $1.43^{-04} \pm 7.59^{-05}$ & $1.34^{-05} \pm 1.08^{-04}$ & $4.99^{-05} \pm 5.83^{-05}$ \\
Xylose & $9.04^{-06} \pm 3.19^{-06}$ & $1.33^{-05} \pm 1.19^{-05}$ & $6.14^{-06} \pm 2.21^{-05}$ & $1.63^{-05} \pm 4.77^{-06}$ \\
\hline
\end{tabular}


Supplemental Table S5.2. Mean quantitative data of the metabolites identified in the symbiont tissues of Montipora capitata grouped according to the Symbiodinium community composition Data are mean relative abundance \pm S.E.M. Labels indicate Symbiodinium composition: $\mathrm{C}=$ clade $\mathrm{C}$-only $(n=20)$; $\mathrm{Cd}=$ clade $\mathrm{C}$ dominant with less abundant clade $\mathrm{D}(n$ $=6) ; \mathrm{Dc}=$ clade $\mathrm{D}$ dominant with less abundant clade $\mathrm{C}(n=11) ; \mathrm{D}=$ clade $\mathrm{D}$-only $(n=3)$.

\begin{tabular}{|c|c|c|c|c|c|c|c|c|c|c|c|}
\hline Metabolite & $\mathbf{C}$ & \pm & S.E.M & Cd & $\underline{+}$ & S.E.M & Dc & \pm & S.E.M & D & \pm S.E.M \\
\hline 1-Hexadecanol & $8.64^{-03}$ & \pm & $1.27^{-03}$ & $6.20^{-03}$ & \pm & $1.23^{-03}$ & $8.57^{-03}$ & \pm & $2.28^{-03}$ & $1.20^{-02}$ & $\pm 3.77^{-03}$ \\
\hline Adenine & $2.67^{-03}$ & \pm & $7.97^{-04}$ & $1.41^{-03}$ & \pm & $4.83^{-04}$ & $3.48^{-03}$ & \pm & $6.87^{-04}$ & $2.41^{-03}$ & $\pm 7.54^{-04}$ \\
\hline Adenosine & $4.56^{-03}$ & + & $2.21^{-03}$ & $2.68^{-03}$ & + & $2.18^{-03}$ & $9.39^{-03}$ & + & $2.62^{-03}$ & $9.62^{-03}$ & $+7.85^{-03}$ \\
\hline Alanine & $2.73^{-01}$ & \pm & $2.96^{-02}$ & $2.69^{-01}$ & \pm & $1.07^{-01}$ & $2.56^{-01}$ & \pm & $3.37^{-02}$ & $2.30^{-01}$ & $\pm 1.04^{-01}$ \\
\hline Aspartic acid & $1.20^{-03}$ & \pm & $2.36^{-04}$ & $8.19^{-04}$ & + & $2.04^{-04}$ & $2.86^{-03}$ & + & $6.81^{-04}$ & $1.65^{-03}$ & $+7.81^{-04}$ \\
\hline Benzoic acid (C4:0) & $6.54^{-03}$ & \pm & $3.18^{-04}$ & $6.91^{-03}$ & \pm & $9.16^{-04}$ & $7.11^{-03}$ & \pm & $2.76^{-04}$ & $6.38^{-03}$ & $\pm 1.11^{-03}$ \\
\hline Campesterol & $7.00^{-03}$ & \pm & $1.68^{-03}$ & $4.51^{-03}$ & \pm & $2.16^{-03}$ & $1.02^{-02}$ & \pm & $2.00^{-03}$ & $1.52^{-02}$ & $\pm 7.98^{-03}$ \\
\hline Cytosine & $2.24^{-04}$ & \pm & $3.03^{-05}$ & $1.94^{-04}$ & \pm & $4.81^{-05}$ & $2.74^{-04}$ & \pm & $3.87^{-05}$ & $2.83^{-04}$ & $\pm 9.46^{-05}$ \\
\hline Decanoic acid (C10:0) & $4.75^{-03}$ & \pm & $1.25^{-03}$ & $2.29^{-03}$ & \pm & $4.59^{-04}$ & $5.19^{-03}$ & \pm & $1.37^{-03}$ & $4.52^{-03}$ & $\pm 3.40^{-03}$ \\
\hline $\begin{array}{l}\text { Docosahexaenoic acid } \\
(\mathrm{C} 22: 6)\end{array}$ & $9.34^{-02}$ & \pm & $1.23^{-02}$ & $7.68^{-02}$ & \pm & $2.17^{-02}$ & $1.27^{-01}$ & \pm & $1.52^{-02}$ & $7.70^{-02}$ & $\pm 1.72^{-03}$ \\
\hline Dodecanoic acid (C12:0) & $3.56^{-03}$ & + & $4.99^{-04}$ & $3.73^{-03}$ & \pm & $1.15^{-03}$ & $4.91^{-03}$ & \pm & $1.14^{-03}$ & $2.14^{-03}$ & $+4.70^{-04}$ \\
\hline Eicosanoic acid (C20:0) & $3.21^{-04}$ & \pm & $3.22^{-05}$ & $3.46^{-04}$ & \pm & $1.17^{-04}$ & $3.84^{-04}$ & \pm & $3.50^{-05}$ & $4.32^{-04}$ & $\pm 1.32^{-04}$ \\
\hline Fumaric acid & $5.27^{-03}$ & \pm & $1.62^{-03}$ & $3.17^{-03}$ & \pm & $1.35^{-03}$ & $4.76^{-03}$ & \pm & $1.41^{-03}$ & $3.49^{-03}$ & $\pm 2.62^{-03}$ \\
\hline Galactonic acid & $3.64^{-01}$ & \pm & $1.01^{-02}$ & $3.63^{-01}$ & \pm & $5.87^{-02}$ & $3.62^{-01}$ & \pm & $1.16^{-02}$ & $3.79^{-01}$ & $\pm 3.70^{-02}$ \\
\hline Galactose & $1.27^{-02}$ & \pm & $6.19^{-03}$ & $5.05^{-03}$ & \pm & $3.67^{-03}$ & $1.82^{-02}$ & \pm & $4.65^{-03}$ & $8.49^{-03}$ & $\pm 6.18^{-03}$ \\
\hline Glucose & $3.53^{-02}$ & \pm & $1.45^{-02}$ & $2.04^{-02}$ & \pm & $1.28^{-02}$ & $6.40^{-02}$ & \pm & $1.38^{-02}$ & $5.97^{-02}$ & $\pm 3.85^{-02}$ \\
\hline Glutamic acid & $7.73^{-04}$ & \pm & $1.10^{-04}$ & $5.11^{-04}$ & \pm & $2.02^{-04}$ & $1.41^{-03}$ & \pm & $2.39^{-04}$ & $8.95^{-04}$ & $\pm 3.07^{-04}$ \\
\hline Glyceric acid & $4.97^{-04}$ & \pm & $1.07^{-04}$ & $2.85^{-04}$ & \pm & $7.93^{-05}$ & $5.11^{-04}$ & \pm & $8.84^{-05}$ & $2.76^{-04}$ & $\pm 1.18^{-04}$ \\
\hline Glycerol & $9.33^{-02}$ & \pm & $1.74^{-02}$ & $4.86^{-02}$ & \pm & $1.21^{-02}$ & $1.71^{-01}$ & \pm & $3.35^{-02}$ & $5.21^{-02}$ & $\pm 1.57^{-02}$ \\
\hline Glycolic acid & $9.58^{-02}$ & \pm & $1.78^{-02}$ & $6.16^{-02}$ & \pm & $1.31^{-02}$ & $9.43^{-02}$ & \pm & $1.15^{-02}$ & $7.49^{-02}$ & $\pm 2.56^{-02}$ \\
\hline Guanine & $3.75^{-03}$ & \pm & $1.08^{-03}$ & $6.51^{-03}$ & \pm & $3.71^{-03}$ & $5.80^{-03}$ & \pm & $1.76^{-03}$ & $3.34^{-03}$ & $\pm 4.06^{-04}$ \\
\hline Hexadecanoic acid C16:0 & $1.81^{-01}$ & \pm & $1.92^{-02}$ & $1.82^{-01}$ & \pm & $5.06^{-02}$ & $2.50^{-01}$ & \pm & $2.26^{-02}$ & $2.14^{-01}$ & $\pm 8.84^{-02}$ \\
\hline myo-Inositol-2-P & $1.52^{-03}$ & \pm & $3.31^{-04}$ & $7.19^{-04}$ & \pm & $2.20^{-04}$ & $1.90^{-03}$ & \pm & $4.66^{-04}$ & $9.41^{-04}$ & $\pm 4.39^{-04}$ \\
\hline myo-Inositol & $1.30^{-02}$ & \pm & $2.79^{-03}$ & $8.83^{-03}$ & \pm & $2.41^{-03}$ & $1.89^{-02}$ & \pm & $2.97^{-03}$ & $1.11^{-02}$ & $\pm 5.39^{-03}$ \\
\hline scyllo-Inositol & $2.18^{-02}$ & \pm & $7.33^{-03}$ & $1.07^{-02}$ & \pm & $2.91^{-03}$ & $2.38^{-02}$ & \pm & $7.00^{-03}$ & $1.88^{-02}$ & $\pm 6.64^{-03}$ \\
\hline Lactic acid & $3.29^{-02}$ & \pm & $2.08^{-03}$ & $5.06^{-02}$ & \pm & $2.15^{-02}$ & $3.50^{-02}$ & \pm & $1.48^{-03}$ & $2.88^{-02}$ & $\pm 2.11^{-03}$ \\
\hline Linoleic acid (C18:2) & $3.62^{-03}$ & \pm & $9.49^{-04}$ & $4.30^{-03}$ & \pm & $1.59^{-03}$ & $6.51^{-03}$ & \pm & $1.15^{-03}$ & $5.08^{-03}$ & $\pm 4.22^{-03}$ \\
\hline Linolenic acid (C18:3) & $1.68^{-01}$ & \pm & $3.09^{-02}$ & $1.22^{-01}$ & \pm & $3.67^{-02}$ & $1.21^{-01}$ & \pm & $2.77^{-02}$ & $1.63^{-01}$ & $\pm 9.08^{-02}$ \\
\hline Mannose & $7.84^{-03}$ & \pm & $4.29^{-03}$ & $2.13^{-03}$ & \pm & $1.37^{-03}$ & $9.16^{-03}$ & \pm & $2.89^{-03}$ & $6.66^{-03}$ & $\pm 5.23^{-03}$ \\
\hline Myristic acid (C14:0) & $3.84^{-02}$ & \pm & $6.22^{-03}$ & $3.03^{-02}$ & \pm & $8.60^{-03}$ & $6.19^{-02}$ & + & $7.49^{-03}$ & $3.76^{-02}$ & $+1.28^{-02}$ \\
\hline Nonanoic acid (C9:0) & $1.70^{-03}$ & \pm & $1.98^{-04}$ & $1.51^{-03}$ & \pm & $2.16^{-04}$ & $1.93^{-03}$ & \pm & $1.86^{-04}$ & $1.51^{-03}$ & $\pm 4.98^{-04}$ \\
\hline Oleic acid (C18:1) & $3.87^{-03}$ & \pm & $6.72^{-04}$ & $6.44^{-03}$ & \pm & $1.87^{-03}$ & $6.26^{-03}$ & \pm & $9.15^{-04}$ & $6.77^{-03}$ & $\pm 4.28^{-03}$ \\
\hline Octanoic acid (C8:0) & $3.87^{-03}$ & \pm & $1.15^{-03}$ & $2.06^{-03}$ & \pm & $3.56^{-04}$ & $4.37^{-03}$ & \pm & $9.65^{-04}$ & $3.04^{-03}$ & $\pm 1.26^{-03}$ \\
\hline Palmitoleic acid (C16:1) & $1.42^{-02}$ & \pm & $3.35^{-03}$ & $1.20^{-02}$ & \pm & $3.78^{-03}$ & $2.57^{-02}$ & \pm & $4.28^{-03}$ & $2.20^{-02}$ & $\pm 1.51^{-02}$ \\
\hline Phosphoric acid & $2.30^{-02}$ & \pm & $3.83^{-03}$ & $1.91^{-02}$ & \pm & $7.57^{-03}$ & $5.71^{-02}$ & \pm & $1.81^{-02}$ & $3.65^{-02}$ & $\pm 1.05^{-02}$ \\
\hline Pyroglutamic acid & $9.13^{-03}$ & \pm & $1.40^{-03}$ & $7.79^{-03}$ & \pm & $1.86^{-03}$ & $1.72^{-02}$ & \pm & $3.29^{-03}$ & $1.77^{-02}$ & $\pm 7.00^{-03}$ \\
\hline Serine & $2.47^{-03}$ & \pm & $3.66^{-04}$ & $2.08^{-03}$ & \pm & $4.52^{-04}$ & $4.25^{-03}$ & \pm & $7.39^{-04}$ & $2.91^{-03}$ & $\pm 1.49^{-03}$ \\
\hline Sucrose & $2.79^{-03}$ & \pm & $4.28^{-04}$ & $4.96^{-03}$ & \pm & $2.46^{-03}$ & $3.24^{-03}$ & \pm & $4.25^{-04}$ & $5.04^{-03}$ & $\pm 2.08^{-03}$ \\
\hline Threonic acid & $9.13^{-04}$ & \pm & $1.80^{-04}$ & $4.99^{-04}$ & \pm & $1.50^{-04}$ & $1.72^{-03}$ & \pm & $4.51^{-04}$ & $1.24^{-03}$ & $\pm 6.91^{-04}$ \\
\hline Threonine & $3.89^{-04}$ & \pm & $6.19^{-05}$ & $4.27^{-04}$ & \pm & $9.98^{-05}$ & $6.94^{-04}$ & \pm & $1.46^{-04}$ & $6.03^{-04}$ & $\pm 2.70^{-04}$ \\
\hline Thymine & $8.06^{-04}$ & \pm & $2.04^{-04}$ & $4.91^{-04}$ & \pm & $1.34^{-04}$ & $1.05^{-03}$ & \pm & $2.25^{-04}$ & $4.68^{-04}$ & $\pm 1.56^{-04}$ \\
\hline Tyrosine & $5.47^{-04}$ & \pm & $1.19^{-04}$ & $4.54^{-04}$ & \pm & $1.31^{-04}$ & $1.67^{-03}$ & \pm & $6.50^{-04}$ & $1.02^{-03}$ & $\pm 6.90^{-04}$ \\
\hline Uracil & $7.79^{-04}$ & + & $1.78^{-04}$ & $6.80^{-04}$ & + & $2.00^{-04}$ & $9.57^{-04}$ & + & $2.17^{-04}$ & $8.98^{-04}$ & $+5.67^{-04}$ \\
\hline
\end{tabular}


Figure S5.1 Montipora capitata host and symbiont metabolite profiles grouped according to reef location. A) PCA analysis of the relative abundance of the metabolites detected in host free pools between the three reef locations (with 95\% confidence regions); B) SAM analysis for the identification of individual metabolites that may vary between the reef locations in the host; C) PCA analysis of the relative abundance of the metabolites detected in symbiont free pools between the three reef locations (with 95\% confidence regions); D) SAM analysis for the identification of individual metabolites that may vary between the reef locations in the symbiont.

A

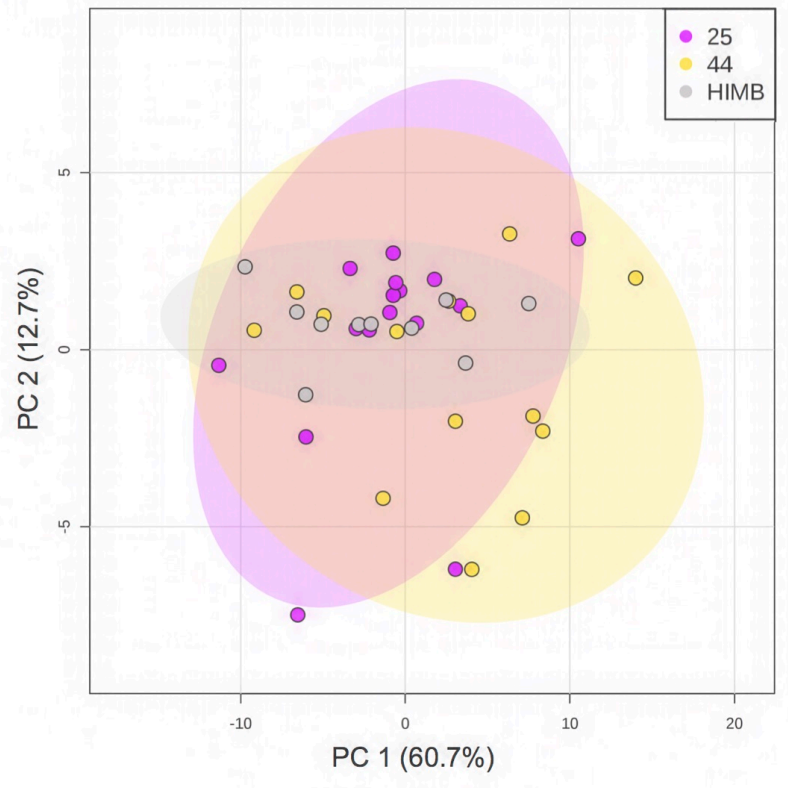

C

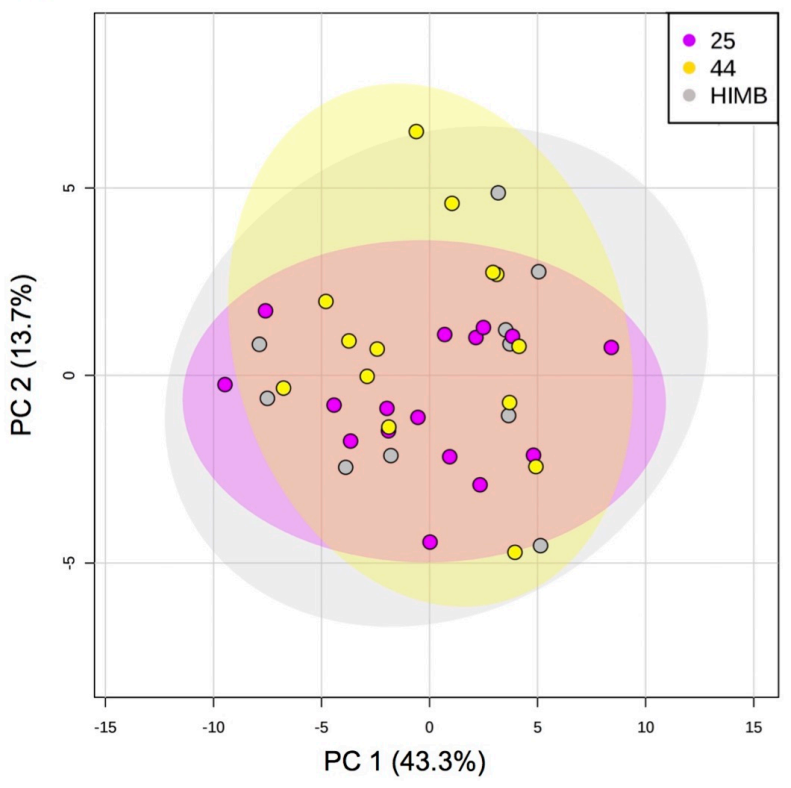

B

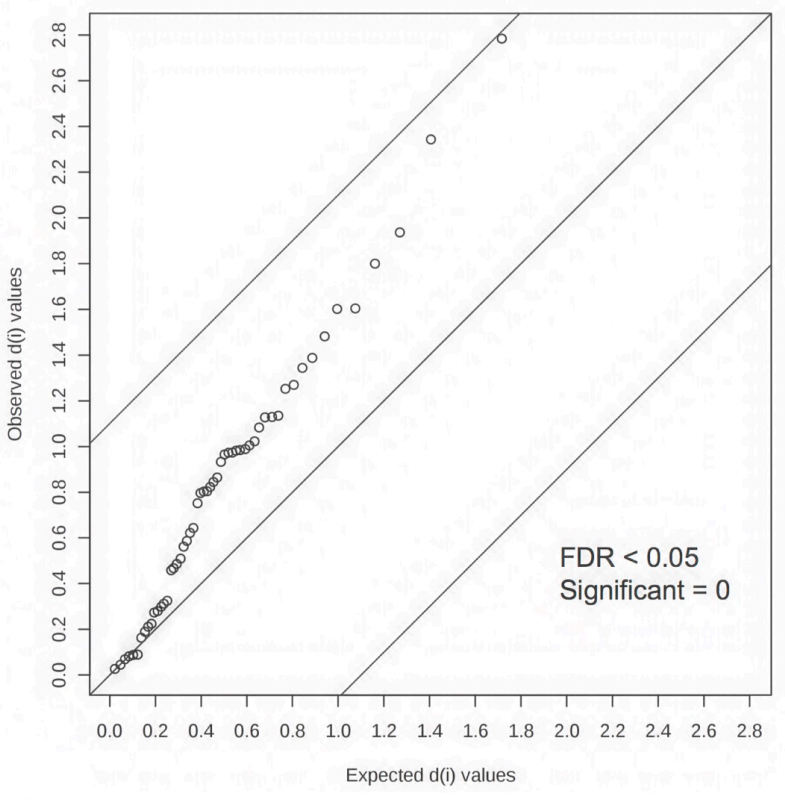

D

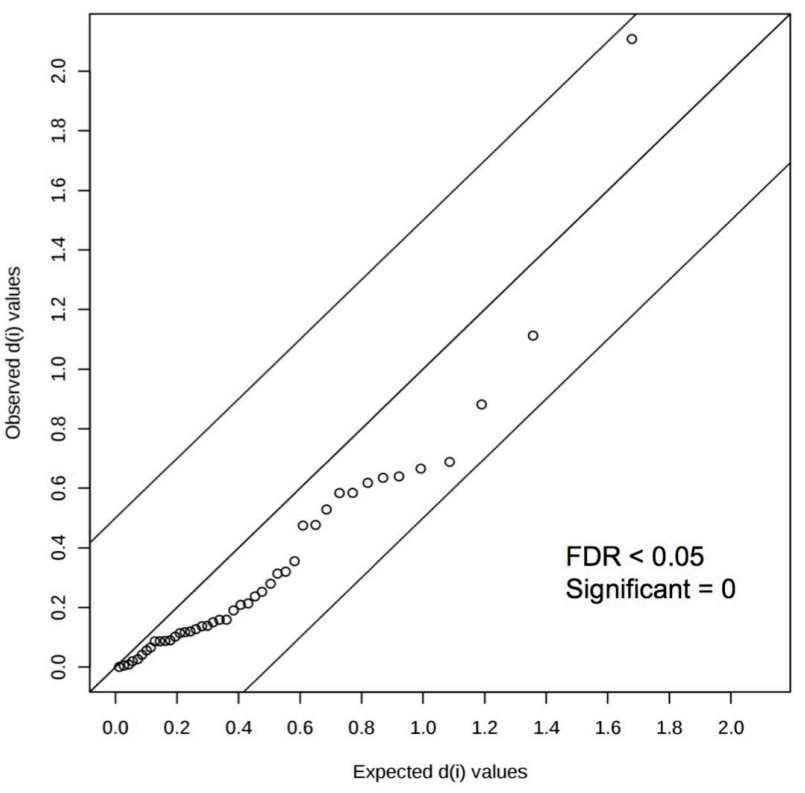




\section{APPENDIX}

This CD contains the following information:

Table A1 A complete list of variance stabilised transcript counts.

Table A2 A list of the 720 differentially expressed genes $($ FDR $<0.1)$.

Table A3 A list of the 686 differentially expressed genes from the host transcriptome including GO terms.

Table A4 A complete list of significantly enriched pathways (FDR $<0.05$ and at least 2 genes or metabolites present) per IMPaLa analysis of heterologous associations against homologous associations.

Figure A1 Putative identification for the Unknown_19.53 labelled compound based on mass isotopomer distribution similarity.

Figure A2 Putative identifications for the Unknown_22.53 and Unknown_28.8 labelled compounds based on mass isotopomer distribution similarity. 


\title{
Menthol-induced bleaching rapidly and effectively provides experimental aposymbiotic sea anemones (Aiptasia sp.) for symbiosis investigations
}

\author{
Jennifer L. Matthews ${ }^{1}$, Ashley E. Sproles ${ }^{1}$, Clinton A. Oakley ${ }^{1}$, Arthur R. Grossman ${ }^{2}$, Virginia M. Weis ${ }^{3}$ and \\ Simon K. Davy ${ }^{1, *}$
}

\begin{abstract}
Experimental manipulation of the symbiosis between cnidarians and photosynthetic dinoflagellates (Symbiodinium spp.) is crucial to advancing the understanding of the cellular mechanisms involved in host-symbiont interactions, and overall coral reef ecology. The anemone Aiptasia sp. is a model for cnidarian-dinoflagellate symbiosis, and notably it can be rendered aposymbiotic (i.e. dinoflagellate-free) and re-infected with a range of Symbiodinium types. Various methods exist for generating aposymbiotic hosts; however, they can be hugely time consuming and not wholly effective. Here, we optimise a method using menthol for production of aposymbiotic Aiptasia. The menthol treatment produced aposymbiotic hosts within just 4 weeks (97-100\% symbiont loss), and the condition was maintained long after treatment when anemones were held under a standard light:dark cycle. The ability of Aiptasia to form a stable symbiosis appeared to be unaffected by menthol exposure, as demonstrated by successful re-establishment of the symbiosis when anemones were experimentally re-infected. Furthermore, there was no significant impact on photosynthetic or respiratory performance of re-infected anemones.
\end{abstract}

KEY WORDS: Cnidarian-dinoflagellate symbiosis, Symbiodinium, Coral reefs

\section{INTRODUCTION}

The symbiosis between corals and dinoflagellate algae of the genus Symbiodinium promotes the success of coral reefs in nutrient-poor tropical seas. However, there are still large gaps in our knowledge about how this symbiosis is established and maintained (Davy et al., 2012; Weis and Allemand, 2009; Weis et al., 2008). Moreover, we do not fully understand how and why this symbiosis breaks down under stress (e.g. bleaching) (Weis, 2008). These knowledge gaps hinder our capacity to understand the function of coral reefs, and to predict how coral reefs might respond to our changing environment.

To elucidate the cellular basis of the coral-dinoflagellate relationship, the symbiotic sea anemone Aiptasia sp. has been widely adopted as a model system because of its robust nature and the ability to produce large populations easily in the laboratory (Weis et al., 2008). Furthermore, Aiptasia sp. can be cleared

\footnotetext{
School of Biological Sciences, Victoria University of Wellington, Wellington 6140, New Zealand. ${ }^{2}$ Department of Plant Biology, The Carnegie Institution, Stanford, CA 94305, USA. ${ }^{3}$ Oregon State University, Department of Integrative Biology, 3029 Cordley Hall, Corvallis, OR 97331, USA

*Author for correspondence (Simon.Davy@vuw.ac.nz)
}

Received 25 July 2015; Accepted 5 November 2015 of symbionts, maintained in an aposymbiotic state for extended periods, and successfully re-infected with a variety of Symbiodinium types (Belda-Baillie et al., 2002; Schoenberg and Trench, 1980; Starzak et al., 2014). The ease of experimental manipulation, combined with the rapidly increasing resolution of genomic, proteomic and metabolic databases for Aiptasia sp., makes this symbiotic anthozoan an ideal candidate for biochemical and genetic experiments that will further our understanding of the cellular processes underlying the cnidarian-dinoflagellate symbiosis (Lehnert et al., 2012; Peng et al., 2010; Sunagawa et al., 2009; Weis et al., 2008).

Currently, the most commonly used method to render anemones (including Aiptasia sp.) aposymbiotic combines temperature stress (heat or cold shock) followed by dark treatment and/or chemical inhibition of photosynthesis with 3-(3,4-dichlorophenyl)1,1-dimethylurea (DCMU) (e.g. Belda-Baillie et al., 2002; Lehnert et al., 2014; Xiang et al., 2013). This is a slow, laborious process that often requires months of preparation (Starzak et al., 2014; Xiang et al., 2013), and may not result in the full eradication of in hospite endosymbionts (Belda-Baillie et al., 2002; Schoenberg and Trench, 1980; Wang and Douglas, 1998).

Recently, Wang et al. (2012) described a method of mentholinduced bleaching in the corals Isopora palifera and Stylophora pistillata. Menthol is known to cause local anaesthetic effects in neuronal and skeletal muscles via blocking voltage-operated sodium channels (Haeseler et al., 2002). This cellular response has led to its use as a marine anaesthetic (Alexander, 1964; Lauretta et al., 2014). Menthol is known to act on a variety of different membrane receptors, including transient receptor potential (TRP) M8, which results in an increase in intracellular $\mathrm{Ca}^{2+}$ concentrations and causes a cold sensation in vertebrates (Hans et al., 2012; McKemy et al., 2002; Okazawa et al., 2000; Peier et al., 2002). As proposed by Wang et al. (2012), the mechanism of menthol-induced bleaching might be attributable to $\mathrm{Ca}^{2+}$-stimulated exocytosis (Pang and Südhof, 2010). Preliminary experiments conducted by Wang et al. (2012) also suggest that menthol inhibition of Symbiodinium photosystem II activity may play a role in the expulsion of the algal cells or the digestion of the Symbiodinium cells by the host. Although the exact mechanism by which menthol induces symbiont expulsion is not yet clear, the findings of Wang and co-authors provide a platform for developing the use of menthol to generate aposymbiotic Aiptasia sp.

We therefore tested the applicability of this approach to Aiptasia sp., in terms of its effectiveness and impact on the capacity for symbiosis re-establishment. In particular, we determined whether menthol can rapidly and effectively produce aposymbiotic anemones, and whether these anemones can be experimentally reinfected with symbiotic dinoflagellates at cell densities similar to 
those of untreated and healthy symbiotic anemones. Ultimately, we describe a method to assist the many researchers around the world who use the Aiptasia model system to further our understanding of the coral-dinoflagellate symbiosis.

\section{MATERIALS AND METHODS}

\section{Experimental organisms}

Symbiotic individuals of Aiptasia sp. $(n=300)$ were harvested from a long-term laboratory stock maintained in $1 \mu \mathrm{m}$ filtered seawater (FSW) at $25^{\circ} \mathrm{C}$, with light provided by AQUA-GLO T8 fluorescent bulbs at $\sim 95 \mu \mathrm{mol}$ photons $\mathrm{m}^{-2} \mathrm{~s}^{-1}$ (12 h:12 h light:dark cycle). Anemones were maintained in $1 \mu \mathrm{m}$ FSW aerated with a Hailea ${ }^{\circledR}$ aquarium air pump at $21 \mathrm{~min}^{-1}$ and at $25^{\circ} \mathrm{C}$ using the $\mathrm{WEIPRO}^{\circledR}$ Temperature Controller MX-1019. These lighting and aquarium conditions were maintained throughout the 19-week experiment. The anemones were evenly divided among the treatments (menthol, cold shock and untreated) and allowed to settle for $72 \mathrm{~h}$ before beginning a 4-week treatment period.

The Symbiodinium culture (ID: FLAp2) was grown at $25^{\circ} \mathrm{C}$ and an irradiance of $100 \mu \mathrm{mol}$ photons $\mathrm{m}^{-2} \mathrm{~s}^{-1}$ on a $12 \mathrm{~h}: 12 \mathrm{~h}$ light:dark cycle. Symbiodinium cells were sub-cultured from a laboratory stock and grown in silica-free f/2 medium (AusAqua Pty, SA, Australia) for 6 weeks before use in the infection study. To genotype the Symbiodinium cells, DNA was extracted as described by Hill et al. (2014) from an algal pellet derived from a $1 \mathrm{ml}$ aliquot of the Symbiodinium suspension. Samples were immediately used for PCR, using the thermal cycling regime and reaction mixture of Hill et al. (2014), and the outer primers ITSintfor2 and ITS2Rev2. PCR products were cleaned with ExoSAP-IT (USB Corporation, $\mathrm{OH}, \mathrm{USA}$ ) and sequenced by the Macrogen Sequencing Service
(Macrogen, Seoul, South Korea). Sequences were aligned with Geneious v. 7.0 (Biomatters, Auckland, NZ) and a BLAST search was carried out against Symbiodinium ITS2 sequences in GenBank. Using this approach, the Symbiodinium genotype was confirmed as B1.

\section{Menthol versus cold-shock treatment}

Menthol (20\% w/v in ethanol; Sigma-Aldrich, Auckland, NZ) was added to $1 \mu \mathrm{m} \mathrm{FSW}$ at a final concentration of $0.19 \mathrm{mmol}^{-1}$. This concentration resulted in successful bleaching without causing mortality. A higher concentration of $0.38 \mathrm{mmol}^{-1}$ as suggested by Wang et al. (2012) was trialled prior to this experiment, but caused mortality of over $50 \%$ of the anemones (J.L.M., unpublished data). The anemones were incubated in the menthol/FSW solution for $8 \mathrm{~h}$, after which the menthol/FSW was removed and the anemones were incubated in $1 \mu \mathrm{m}$ FSW for $16 \mathrm{~h}$. DCMU $\left(100 \mathrm{mmol}^{-1}\right.$ dissolved in EtOH, Sigma-Aldrich) was added to a final concentration of $5 \mu \mathrm{mol} \mathrm{l}^{-1}$ to prevent nuisance algal blooms and to limit the reestablishment of any residual symbiont cells by inhibiting photosynthesis. This 24-h cycle was repeated for four consecutive days. The 8-h menthol/FSW incubation occurred during the 12-h light period. The 4-day treatment was repeated after a 3-day break, during which anemones were maintained under the lighting and temperature conditions stated above, with biweekly feeding with Artemia sp. nauplii.

For cold shock, the water was replaced with $4^{\circ} \mathrm{C} 1 \mu \mathrm{m}$ FSW, and placed in a refrigerator at $4^{\circ} \mathrm{C}$ for $4 \mathrm{~h}$. Afterwards, the water was replaced with $25^{\circ} \mathrm{C} 1 \mu \mathrm{m}$ FSW containing a final concentration of $50 \mu \mathrm{mol} \mathrm{l}^{-1}$ DCMU $\left(100 \mathrm{mmol}^{-1}\right.$ dissolved in EtOH, SigmaAldrich) and held under the lighting and aquarium conditions
A

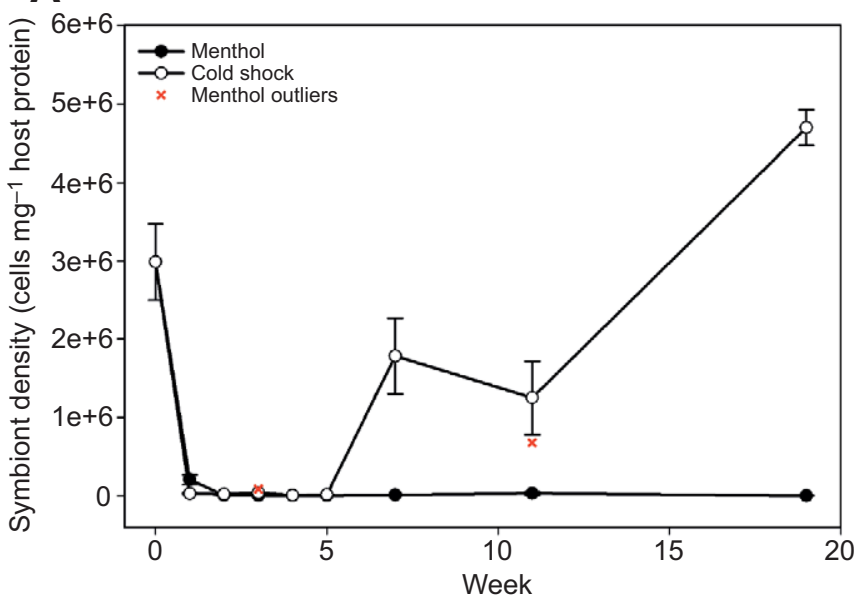

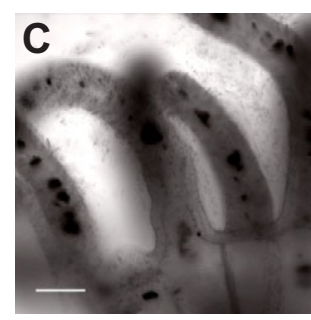

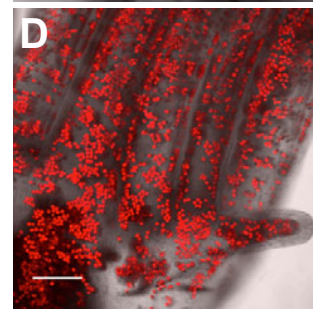

Fig. 1. Effects of menthol and cold shock on algal cell density in Aiptasia sp. (A) Density of Symbiodinium cells in menthol-treated (closed circles) and cold-shocked (open circles) anemones over a 19-week period. The anemones were treated for the first 4 weeks, then left to recover in regular filtered seawater for the remaining 15 weeks. Outliers (defined as exceeding $1.5 \times$ interquartile range) were detected in the menthol-treated anemones at weeks 4 and 11 , and are indicated by a red cross. Values are mean \pm s.e.m.; $n=5$. (B) Confocal microscopy images of anemones during menthol treatment and cold shock (weeks 1-4). Yellow arrows indicate Symbiodinium cells in host tissues. $(C, D)$ Confocal fluorescence images of mentholtreated and cold-shocked anemones, respectively, 15 weeks post-treatment (i.e. week 19). Scale bars are $100 \mu \mathrm{m}$.
B

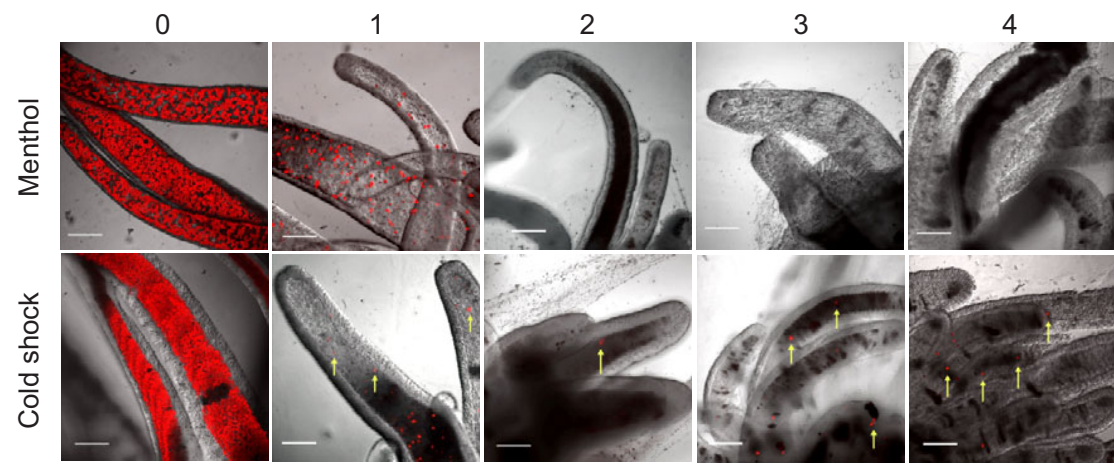


described above. The anemones were incubated under these conditions for 3 days, and the treatment was then repeated. The anemones were unfed for the duration of the cold shock, as per the protocol described by Steen and Muscatine (1987). Anemones were cold shocked a total of eight times (twice per week) during the treatment period.

After the 4-week treatment period, all anemones were maintained in $1 \mu \mathrm{m} \mathrm{FSW}$ at $25^{\circ} \mathrm{C}$ under the irradiance regime described above, with biweekly feeding, for an additional 15 weeks. Their symbiotic status was assessed via confocal fluorescence microscopy and cell density estimates (described below) at weeks 1-5, 7, 9, 11 and 19.

The untreated group of anemones was kept under the same thermal and light regimes as the menthol-treated and cold-shocked anemones, with biweekly feeding throughout the entire 19-week experiment. They were assessed for symbiont density at the same time points as the experimental anemones, to obtain an average symbiont density of untreated, healthy anemones.

\section{Re-infection of aposymbiotic anemones}

The capacity to re-infect menthol-treated anemones with algal symbionts was tested; this was not possible with cold-shocked anemones because they were not fully aposymbiotic. Anemones were infected with cultured homologous Symbiodinium type B1, i.e. symbionts originally isolated from Aiptasia sp. A drop of concentrated Symbiodinium suspension $\left(3 \times 10^{6}\right.$ cells $\left.\mathrm{ml}^{-1}\right)$ was pipetted onto the oral disc of each anemone, followed by a drop of Artemia sp. nauplii to invoke a feeding response and encourage Symbiodinium uptake (Davy et al., 1997). This process was repeated $4 \mathrm{~h}$ later, and the water was replaced with $1 \mu \mathrm{m}$ FSW $8 \mathrm{~h}$ after the second infection.

\section{Confocal microscopy}

Whole anemones from each treatment were examined at the same time points as cell density assessments by confocal microscopy (Olympus Provis AX70, at $100 \times$ magnification) to detect the chlorophyll autofluorescence of any dinoflagellates. Anemones were selected at random from each treatment, and placed in a 'relaxation solution' [50\% FSW, 50\% $0.37 \mathrm{~mol} \mathrm{l}^{-1}$ magnesium chloride $\left.\left(\mathrm{MgCl}_{2}\right)\right]$ for $15 \mathrm{~min}$ before being moved to a FluoroDish ${ }^{\mathrm{TM}}$ glass-bottom confocal dish (World Precision Instruments, FL, USA). The autofluorescence emission of the Symbiodinium cells was excited using a $559 \mathrm{~nm}$ laser and captured at $647 \pm 10 \mathrm{~nm}$. This was performed to check the rates of bleaching and re-infection, as well as to detect any background Symbiodinium populations in the post-treatment anemones.

\section{Symbiont density and protein content}

Symbiodinium populations were quantified with a haemocytometer and light microscope $(100 \times$ magnification; $n=8$ replicate counts per sample) using whole-anemone homogenate $(n=5)$. Cell density was normalised to soluble protein content, measured via the Bradford assay (Bradford, 1976). Cell density was measured at weeks 1-5, 7, 9, 11 and 19.

\section{$\mathrm{O}_{2}$ flux}

Fifteen weeks after reinfection, maximum photosynthetic and dark respiratory $\mathrm{O}_{2}$ fluxes were measured to compare re-infected menthol-treated anemones against untreated anemones $(n=4)$. A FIBOX 3 fibre optic oxygen transmitter and oxygen probe (PreSens, Germany) were used as described by Starzak et al. (2014). The respiration rate $\left(\mathrm{ml} \mathrm{O}_{2} \mathrm{~h}^{-1}\right)$ was measured for $30 \mathrm{~min}$ in the dark, after which the rate of net photosynthesis was measured for $30 \mathrm{~min}$ at an irradiance of $\sim 100 \mu \mathrm{mol}$ photons $\mathrm{m}^{-2} \mathrm{~s}^{-1}$. Net photosynthesis and respiration were corrected for background levels using a FSWonly control, and gross photosynthesis was calculated by the addition of net photosynthesis to dark respiration (Muscatine et al., 1981). The average ratio of gross photosynthesis to respiration (P:R) for $24 \mathrm{~h}$ was calculated for each anemone, by assuming a $12 \mathrm{~h}: 12 \mathrm{~h}$ light:dark cycle and that respiration was constant in the light and dark, and the average ratio for each treatment was calculated.

\section{Statistical tests}

The non-parametric Mann-Whitney $U$-test was used to test for differences between treatments with respect to symbiont density (SPSS statistical software, v. 20, IBM Corporation). Separate analyses were run for the bleaching and post-bleaching periods.

A one-way ANOVA was performed on the photosynthetic rate, respiration rate and log-transformed $\mathrm{P}: \mathrm{R}$ values to determine whether significant differences were present between the re-infected menthol-treated and untreated symbiotic anemones at the end of the 19-week experimental period.

\section{RESULTS AND DISCUSSION}

Menthol treatment induced $97-100 \%$ symbiont loss within 14-28 days, with $60 \%$ of anemones losing all symbionts. In comparison, cold shock never induced $100 \%$ loss within the same
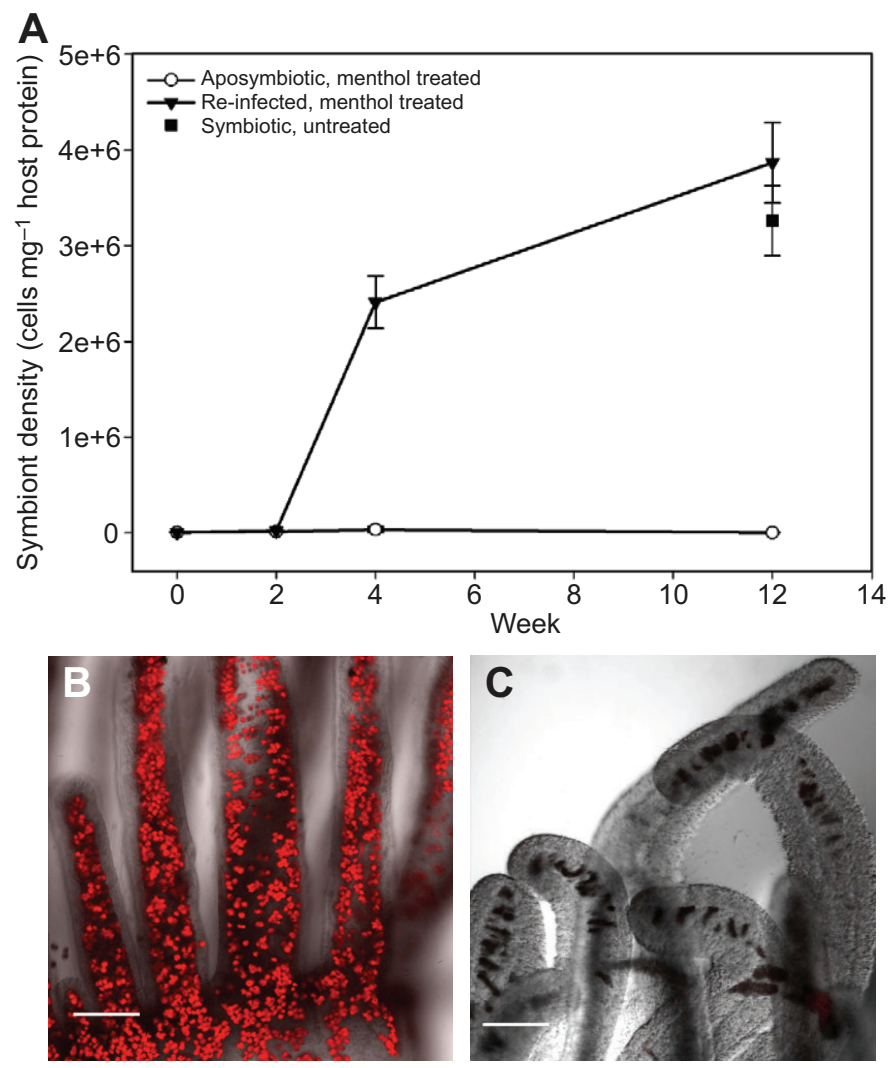

Fig. 2. Repopulation of menthol-treated Aiptasia sp. with algal symbionts (A) Symbiont cell density over a 12-week period after anemones were experimentally re-infected with the homologous Symbiodinium type B1 (closed triangles). Also shown are symbiont densities in symbiotic, untreated anemones (closed square) and menthol-treated anemones that were not experimentally re-infected (open circles). Values are means \pm s.e.m.; $n=5$. $(\mathrm{B}, \mathrm{C})$ Confocal images showing, respectively, the re-established symbiont population 12 weeks after reinfection versus tissues in a non-infected aposymbiotic anemone. Scale bars are $100 \mu \mathrm{m}$. 
time frame (Fig. 1A,B), and any residual symbionts in the mentholtreated anemones did not re-establish a full symbiosis once returned to normal seawater conditions over the course of our experiment (Fig. 1A,C).

Although statistically, the patterns of symbiont loss during weeks 1-4 were not different between the menthol and cold-shock treatments (Mann-Whitney $U=113, \quad$ d.f. $=4, \quad P=0.423, \quad n=5$; Fig. 1A), only the menthol-treated anemones successfully maintained aposymbiotic status in the longer term. In both treatments, symbiont density remained near zero during the first week of recovery (weeks 4-5; Fig. 1A); however, subsequently there was a significant difference between the cell densities of menthol- and cold-shock-treated anemones (Mann-Whitney $U=35$, d.f. $=4$, $P<0.0005 ; n=5)$, with the menthol-treated anemones remaining mostly symbiont-free $(0-0.05 \%$ of pre-bleaching density) and the cold-shocked anemones becoming fully re-populated (weeks 5-9; Fig. 1A). Confocal microscopy confirmed these measurements (Fig. 1C). Menthol treatment therefore has considerable potential for the rapid and effective generation of aposymbiotic Aiptasia sp.

One key use for aposymbiotic Aiptasia sp. is for experimental work that involves re-infection with symbiotic algae of different types and from different host species. It was therefore important to test the infectivity of the menthol-treated anemones, and establish whether there were any significant long-term impacts of menthol treatment versus no treatment on physiological performance (photosynthesis and respiration) of the re-infected anemones. When infected with Symbiodinium cells (ITS2 type B1), symbiont density increased slowly during the initial 2 weeks, followed by a rapid increase during weeks 2-12 (Fig. 2A). Confocal microscopy confirmed that mentholtreated anemones were fully repopulated after 12 weeks (Fig. 2B). The infection pattern and maximum cell density were similar to those of other re-infection studies using heat- and cold-shock bleached Aiptasia and type B1 Symbiodinium, where a steep increase in cell density was observed in the first 4 weeks, followed by stabilisation (Belda-Baillie et al., 2002; Schoenberg and Trench, 1980; Starzak et al., 2014). Additionally, the photosynthetic rate $(P=0.432 ; n=4)$, respiration rate $(P=0.347 ; n=4)$ and ratio of photosynthesis to respiration $(P=0.368 ; n=4)$ in these anemones were similar to those of untreated symbiotic anemones. This indicates that their capacity to reform a functional symbiosis was not compromised, further highlighting the value of this method.

As expected, given its anaesthetic properties (Alexander, 1964; Lauretta et al., 2014), menthol induced tentacle relaxation and unresponsiveness in anemones for the duration of the treatment. Once the seawater was replaced with regular FSW, the anemones were able to regain full movement and feeding capabilities within $\sim 15 \mathrm{~min}$. This is in stark contrast to the residual effects of cold shock on anemones, where long-lasting tentacle retraction and occasional mortality were observed.

In summary, we provide a rapid and effective method for the generation of aposymbiotic Aiptasia sp. that will facilitate studies of symbiosis establishment and maintenance, host-symbiont recognition, and symbiosis function with this model system. In particular, menthol treatment has the potential to rapidly create large stocks of aposymbiotic anemones without compromising their infectivity. Ultimately, this method will help to accelerate the rate of discovery as we try to better understand the function of coral reefs, and their response and adaptive capacity in the face of climate change.

\section{Acknowledgements}

We are grateful to Malindi Gammon and Josh Brian for laboratory assistance.

\section{Competing interests}

The authors declare no competing or financial interests.

\section{Author contributions}

J.L.M., A.E.S., C.A.O., A.R.G., V.M.W. and S.K.D. conceived and designed the experiments. J.L.M. and A.E.S. carried out the experiments and subsequent data analysis. J.L.M., A.E.S., C.A.O., A.R.G., V.M.W. and S.K.D. wrote the manuscript.

\section{Funding}

This work was supported by a grant from the Royal Society of New Zealand Marsden Fund (grant no. 1202 awarded to S.K.D., A.R.G. and V.M.W.)

\section{References}

Alexander, R. M. (1964). Visco-elastic properties of the mesogloea of jellyfish. J. Exp. Biol. 41, 363-369.

Belda-Baillie, C. A., Baillie, B. K. and Maruyama, T. (2002). Specificity of a model cnidarian-dinoflagellate symbiosis. Biol. Bull. 202, 74-85.

Bradford, M. M. (1976). A rapid and sensitive method for the quantitation of microgram quantities of protein utilizing the principle of protein-dye binding. Anal. biochem. 72, 248-254.

Davy, S., Turner, J. and Lucas, I. (1997). The nature of temperate anthozoandinoflagellate symbioses. In Proc. 7th Int. Coral Reef Symp., vol. 2, pp. 1307-1312.

Davy, S., Turner, J. and Lucas, I. (1997). The nature of temperate anthozoandinoflagellate symbioses. In Proc. 7th Int. Coral Reef Symp., vol. 2 pp. 1307-1312.

Davy, S. K., Allemand, D. and Weis, V. M. (2012). Cell biology of cnidariandinoflagellate symbiosis. Microbiol. Mol. Biol. Rev. 76, 229-261.

Haeseler, G., Maue, D., Grosskreutz, J., Bufler, J., Nentwig, B., Piepenbrock, S., Dengler, R. and Leuwer, M. (2002). Voltage-dependent block of neuronal and skeletal muscle sodium channels by thymol and menthol. Eur. J. Anaesthesiol. 19, 571-579.

Hans, M., Wilhelm, M. and Swandulla, D. (2012). Menthol suppresses nicotinic acetylcholine receptor functioning in sensory neurons via allosteric modulation. Chem. Senses 37, 463-469.

Hill, R., Fernance, C., Wilkinson, S. P., Davy, S. K. and Scott, A. (2014). Symbiont shuffling during thermal bleaching and recovery in the sea anemone Entacmaea quadricolor. Mar. Biol. 161, 2931-2937.

Lauretta, D., Häussermann, V., Brugler, M. R. and Rodríguez, E. (2014) Isoparactis fionae sp. nov. (Cnidaria: Anthozoa: Actiniaria) from Southern Patagonia with a discussion of the family Isanthidae. Organ. Divers. Evol. 14, 31-42.

Lehnert, E. M., Burriesci, M. S. and Pringle, J. R. (2012). Developing the anemone Aiptasia as a tractable model for cnidarian-dinoflagellate symbiosis: the transcriptome of aposymbiotic A. pallida. BMC Genomics 13, 271.

Lehnert, E. M., Mouchka, M. E., Burriesci, M. S., Gallo, N. D., Schwarz, J. A. and Pringle, J. R. (2014). Extensive differences in gene expression between symbiotic and aposymbiotic cnidarians. G3 4, 277-295.

McKemy, D. D., Neuhausser, W. M. and Julius, D. (2002). Identification of a cold receptor reveals a general role for TRP channels in thermosensation. Nature 416 , 52-58.

Muscatine, L., McCloskey, L. and Marian, R. (1981). Estimating the daily contribution of carbon from zooxanthellae to coral animal respiration. Oceanography 26, 601-611.

Okazawa, M., Terauchi, T., Shiraki, T., Matsumura, K. and Kobayashi, S. (2000). I-Menthol-induced $\left[\mathrm{Ca}^{2+}\right] \mathrm{i}$ increase and impulses in cultured sensory neurons. Neuroreport 11, 2151-2155.

Pang, Z. P. and Südhof, T. C. (2010). Cell biology of $\mathrm{Ca}^{2+}$-triggered exocytosis. Curr. Opin. Cell Biol. 22, 496-505.

Peier, A. M., Moqrich, A., Hergarden, A. C., Reeve, A. J., Andersson, D. A., Story, G. M., Earley, T. J., Dragoni, I., Mclntyre, P. and Bevan, S. (2002). A TRP channel that senses cold stimuli and menthol. Cell 108, 705-715

Peng, S. E., Wang, Y. B., Wang, L. H., Chen, W. N. U., Lu, C. Y., Fang, L. S. and Chen, C. S. (2010). Proteomic analysis of symbiosome membranes in Cnidariadinoflagellate endosymbiosis. Proteomics 10, 1002-1016.

Schoenberg, D. A. and Trench, R. K. (1980). Genetic variation in Symbiodinium (= Gymnodinium) microadriaticum Freudenthal, and specificity in its symbiosis with marine invertebrates. III. Specificity and infectivity of Symbiodinium microadriaticum. Proc. R. Soc. B Biol. Sci. 207, 445-460.

Starzak, D. E., Quinnell, R. G., Nitschke, M. R. and Davy, S. K. (2014). The influence of symbiont type on photosynthetic carbon flux in a model cnidariandinoflagellate symbiosis. Mar. Biol. 161, 711-724

Steen, R. G. and Muscatine, L. (1987). Low temperature evokes rapid exocytosis of symbiotic algae by a sea anemone. Biol. Bull. 172, 246-263.

Sunagawa, S., Wilson, E. C., Thaler, M., Smith, M. L., Caruso, C., Pringle, J. R., Weis, V. M., Medina, M. and Schwarz, J. A. (2009). Generation and analysis of transcriptomic resources for a model system on the rise: the sea 
anemone Aiptasia pallida and its dinoflagellate endosymbiont. BMC Genomics 10, 258.

Wang, J. and Douglas, A. (1998). Nitrogen recycling or nitrogen conservation in an alga-invertebrate symbiosis? J. Exp. Biol. 201, 2445-2453.

Wang, J. T., Chen, Y. Y., Tew, K. S., Meng, P. J. and Chen, C. A. (2012).

Physiological and biochemical performances of menthol-induced aposymbiotic corals. PLoS One 7, e46406.

Weis, V. M. (2008). Cellular mechanisms of Cnidarian bleaching: stress causes the collapse of symbiosis. J. Exp. Biol. 211, 3059-3066.
Weis, V. M. and Allemand, D. (2009). Physiology. What determines coral health? Science 324, 1153-1155.

Weis, V. M., Davy, S. K., Hoegh-Guldberg, O., Rodriguez-Lanetty, M. and Pringle, J. R. (2008). Cell biology in model systems as the key to understanding corals. Trends Ecol. Evol. 23, 369-376.

Xiang, T., Hambleton, E. A., DeNofrio, J. C., Pringle, J. R., Grossman, A. R. and Lin, S. (2013). Isolation of clonal axenic strains of the symbiotic dinoflagellate Symbiodinium and their growth and host specificity. J. Phycol. 49, 447-458. 